\title{
Certain behaviors:
}

\section{Response selection and certainty-related processing in humans and rhesus monkeys}

Dissertation

zur Erlangung des mathematisch-naturwissenschaftlichen Doktorgrades

"Doctor rerum naturalium"

der Georg-August-Universität Göttingen

im Promotionsprogramm Behavior and Cognition

der Georg-August University School of Science (GAUSS)

vorgelegt von

\section{Caio Margarido Moreira}

aus Ribeirão Preto, Brasilien

Göttingen, 2016 


\section{Thesis Committee:}

Dr. Igor Kagan (Supervisor)

Decision and Awareness Group, CNL, German Primate Center, Goettingen

Prof. Dr. Julia Fischer

Cognitive Ethology Laboratory, German Primate Center, Goettingen

Prof. Dr. Michael R. Waldmann

Department of Psychology, University of Goettingen, Goettingen

\section{Members of the Examination Board:}

Referee: Dr. Igor Kagan

Decision and Awareness Group, CNL, German Primate Center, Goettingen

2nd Referee: Prof. Dr. Julia Fischer

Cognitive Ethology Laboratory, German Primate Center, Goettingen

\section{Further members of the Examination Board:}

Prof. Dr. Michael R. Waldmann

Department of Psychology, University of Goettingen, Goettingen

Prof. Dr. Melanie Wilke

Department of Cognitive Neurology, UMG, Goettingen

Dr. Roberto Goya-Maldonado

The Systems Neuroscience and Imaging in Psychiatry (SNIP) Lab, UMG, Goettingen

Prof. Dr. York Hagmayer

Department of Psychology, University of Goettingen, Goettingen 


\section{I hereby declare that I have written the thesis entitled:}

"Certain behaviors: response selection and certainty-related processing in humans and rhesus monkeys"

Submitted for the award of "Doctor rerum naturalium", independently and with no other sources and aids than quoted

Goettingen, June 2016

Signature 
to Cláudia 


\section{Acknowledgements}

I am very fortunate because I have a huge amount of primates to say thank you to!

Thank you Claudia for sharing your life with me. Claudia is a sweet, good and admirable human being. More than that, she is a strong, beautiful and intelligent woman and mother. Having her by my side amazes me, makes me stronger and allows me to try my best. Thank you for leaving behind so many important things in order to allow me to dream. I'm still dreaming... and I hope we'll keep dreaming together.

I also would like to thank Dr. Igor Kagan for his careful supervision and teaching. Igor brings together the nicest combination of human features that a scientist can have: methodological rigor, intelligence and a childish enthusiasm for knowledge. His dedication to science is impressive and contagious, and personally he is one of the nicest and most honest persons I have ever met. Thank you also for all the support you provided for my ideas, goals and for my family.

I thank the examination board of my thesis, especially Prof. Dr. Julia Fischer and Prof. Dr. Michael Waldmann for providing guidance and fruitful discussions during the thesis committee meetings.

I thank Bacchus and Curius. It was amazing to have the opportunity to do science with such wonderful creatures. I know they were not able to choose, but I thank them anyway for allowing me to do my experiments. I hope I was as nice to them as they deserved. And I hope I was worthy of their time.

I thank the Brazilian government, in special the program Science Without Borders, for the financial support of my PhD studies.

I thank the Deustches Primatenzentrum (German Primate Center) and all the colleagues I have there for supporting my $\mathrm{PhD}$ as a whole. In particular, I thank Prof. Dr. Stefan Treue for providing me direct and indirect support.

I thank the Department of Cognitive Neurology for the opportunity for learning and doing my experiments with fMRI. In particular, Prof. Dr. Melanie Wilke.

I thank the Behavior and Cognition PhD program, especially Dr. Schloegl and Dr. Jürgens for the support and advices, and Katja Brodmann for sharing the experience. 
I also thank:

- My human subjects. I'm sure they bet they were really helping me! They were right!

- Ira Panolias for her happiness and for teaching me how to work with monkeys.

- My colleagues from the Decision and Awareness Group (German Primate Center) and from the Department of Cognitive Neurology for teaching me science on a daily basis and for the nice company. In particular: my friend Danial Arabali for being so kind and for having this artistic and interesting view of the world; Malte Köster for receiving me in the group and teaching my first Matlab steps; Lydia Gibson for showing me Germany by behaving; Katie Williams for being brave; Yuranny Cabral and Carsten Schmidt-Samoa for rocking with fMRI.

- My student Max Rollwage for developing scientific ideas with me, for being so intelligent and autonomous, and for "shaving the machado".

- My student Kristin Kaduk for collecting different data sets, and for being so dedicated to the methodological aspects of the work we did together.

- ITs, in particular Hendrik Eichenauer.

- Dr. Sennhenn-Reulen for the statistical support.

- My former supervisors, Prof. Dr. Marcus Lira Brandão and Prof. Dr. Patrícia Izar, for giving me advices that I still use constantly.

- My friends of science and music: Katharina, Antonino, Jonathan, Nuno and Eduardo for trying to keep up with my rhythm and tune, in multiple aspects.

- All my friends and family from Brazil, whom I'm very eager to meet more often again. In special Rafael Ruggiero and Lucas Peternelli the main supporters of my "going abroad plans".

- My friends from soccer. In special Dmitry and Sergey, and their Russian spirit.

- My brothers: Eduardo, Lucas, Ricardo, Plínio, Florian and Vinicius; and sisters: Thaís, Tati, Luísa, Everlin and Olga, for being my family in Göttingen. In special Ricardo Merino for receiving Claudia and me in Göttingen so carefully.

Lastly, I thank my daughter, Luisa, for being. And, by doing so, be so revealing about the world, herself and me. And also my close family, the Drs. Moreira: Dr. Marcos, Dr. Rita, Dr. Helena, Dr. Carol (also Tico, Teco and Xaxim), since their support and guidance are essential in all kinds of ways, in particular for my experience of becoming a father and my pursuit of the scientific career. 


\section{Thesis content}

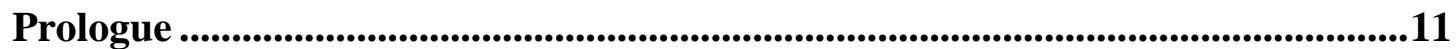

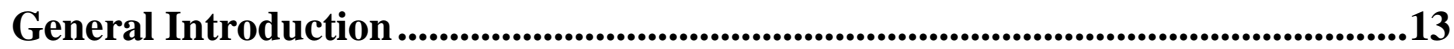

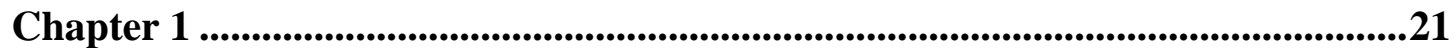

Chapter 2 ..............................................................................................................................................663

Chapter 3 ..................................................................................................................................125

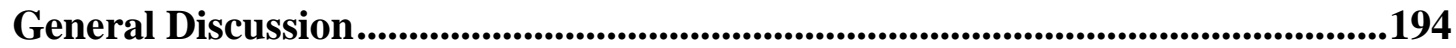

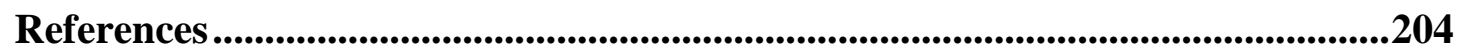

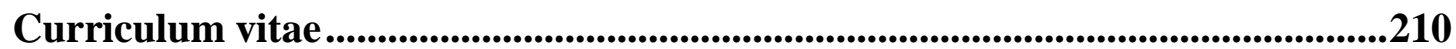




\section{Prologue}

As any thesis, this one is a consequence of several decisions the writer (me) took during his life when he was interacting with the environment that surrounds him. In my case, this environment was mainly influenced by family, friends, professors, musicians, scientists, writers and other idols, and had unique geographical and sociological characteristics.

It has been Brazil. More recently it became also Germany.

Among the innumerous events that led to this thesis, there are three professional ones that I would like to mention:

1) Back in 2008, I was studying wild capuchin monkeys in the Brazilian Atlantic Forest. I was recording the behavior of a specific group of monkeys and collecting their feces to measure stress and reproduction-related hormones. Although most of the fun came from the interactions with juveniles, the behavior of the alpha male (Benjamin) was by far the most interesting one. During a late afternoon, Benjamin was standing in the branch of a very high tree, trying to figure out (I imagine) where he, and consequently the rest of the capuchin group, would sleep. He kept standing there for some minutes. During this time I could not avoid thinking: What is going on in his mind? How does he decide for this or that tree? What influences his decisions?

2) Some of the most important researchers in the field of decision-making were in Goettingen for the Bernstein Conference in 2014. Among them, Paul Cisek and Michael Shadlen. During one afternoon they were at the German Primate Center meeting researchers and students from the Neuroscience section. At a certain point of a scientific discussion, Paul Cisek said that animals' brains were built to generate actions; the brain structure serves action-directed processing; and this is the framework we should use to address questions related to our behavior and to the system that supports it.

3) After being introduced to consciousness-related studies by Dr. Kagan, I became more and more interested in metacognition. At certain point, I read 
Kepecs and Mainen (2012) paper (A computational framework for the study of confidence in humans and animals, Philosophical Transactions of the Royal Society of London B: Biological Sciences, 367, 1322-1337). The idea that "probabilistic reasoning is something that nervous systems do as a matter of their construction" really struck me. The authors suggest that our cognitive processes bring a "p-value" with them that signalizes, when read out, their reliability. According to Kepecs and Mainen, this happens in several animal species. 


\section{General Introduction}

Animal behavior is amazing because of its variety and complexity. Researchers have been studying this topic under different levels of analyses which can be summarized in "proximate causes" (i.e. genetic-developmental and sensory-motor mechanisms) and "ultimate causes" (i.e. selective processes and historical pathways leading to the current behavioral traits; Alcock, 1993; Sherman, 1988; Tinbergen, 1963). The present work focuses on the neurobiology of decision-making. Specifically, it focuses on the sensory-motor mechanisms of decision-making and certainty evaluation, as well as on the modification of interhemispheric processing and flexible responses selection over the course of primate evolution.

Classic studies in cognitive psychology proposed that the center of the executive system is located in the frontal lobes (e.g. Shallice et al., 1982). However, recent studies suggest that the neural mechanisms involved in decision-making are also associated with neural activity in cortical and subcortical regions previously related exclusively to sensorimotor control (e.g. Klaes et al., 2011; Komura et al., 2013). This distributed proposition suggests that the brain inherently and ubiquitously processes multiple sources information in order to create actions (Shadlen et al., 2008), or even implements multiple action options and decides between them already within the sensorimotor system, constructing a distributed consensus among many levels or representations (Cisek, 2012).

According to this action-centered point of view, the primary goal of sensory processing is not the extraction of objects features, but building up (accumulating) evidence that supports actions. It does not mean that animals cannot make purely abstract decisions. Certainly this is not the case for humans, whose reasoning complexity can be associated with many abstract activities. But it means that most, if not all, neural circuits are actually constructed during evolution to process information in order to generate responses that allow interactions with the external environment (Shadlen et al., 2008; Cisek, 2012). For example, some areas of the parietal lobule can encode the force and the velocity of effector-specific responses (as saccades or reaches) and, at the same time, process the reliability of the perceptual evidence that supports those actions (Shadlen et al., 2008; Kiani and Shadlen, 2009). Moreover, using functional imaging, Filimon et al. (2013) showed that as soon as humans have information about which effector (hand or eye) they should employ to 
report perceptual decisions, not only areas in the frontal cortex encode these decisions, but also effector-specific parietal areas start preparing motor responses under the modulation of the perceptual signal strength.

The existence of such versatile and "embodied" processing provides the basis of my investigation. Initially, it defined my methodological approach, since I used fMRI to investigate the activity of the entire human and monkey brains in order to understand the relationship between activation patterns and behavior as a whole (considering methodological limitations). But more importantly, this distributed processing framework brought to my attention one of the most ubiquitous cognitive processes that animals have: certainty readouts.

Certainty (or confidence) can be defined as the reliability of the evidence that supports cognitive processes such as decision-making (Ma and Jazayeri, 2014). Recently, certainty evaluation has been approached using a well-developed computational framework and suggested to be a fundamental brain function across species (Kepecs and Mainen, 2012). Considering that nervous systems inherently perform probabilistic "reasoning" (Zemel et al., 1998; Mamassian et al., 2002; Knill and Pouget, 2004), certainty readouts are thought to provide, in several animal species, confidence intervals about cognitive processes in a similar way that statistical p-values are computed for a hypothesis test (Kepecs and Mainen, 2012).

Moreover, the inherent stochasticity of sensory processing in the nervous system also influences the knowledge individuals have about the state of the world, including the state of their own body (Mamassian et al., 2002; Shadlen et al., 2008). Consequently, probabilistic inferences about the variables that describe the external and the internal environments ("beliefs") might be as important as the sensory evidence itself (Ma and Jazayeri, 2014). Interestingly, animals are able to behave almost optimally assessing subjective probabilities under conditions of such noisy environment (Pouget et al., 2016). Optimality here concerns another important aspect of certainty evaluation: the association between belief and utility. This association is especially important for reward-based decisions since, in this context, certainty can be also defined as the individual's belief that the chosen action maximizes utility (De Martino et al. 2012). 
Very first experiments about confidence in humans date from the end of the nineteenth century (Peirce \& Jastrow, 1884). Since then, innumerous methods were developed to measure certainty. Humans are most often asked to report their certainty, or confidence, explicitly, and their reports demonstrate high correlation with performance accuracy (e.g. Boldt and Yeung, 2015; Charles et al., 2013; Fleming et al., 2012; Hebart et al., 2014; Heereman et al., 2015). Clearly, other animals and patients with language-related impairments cannot provide verbal-based confidence reports. For them, more sophisticated tasks were designed to elicit implicit reports of confidence (for review see Kepecs and Mainen, 2012).

Although this still remains a very debatable issue, some researchers (including myself) came to the conclusion that implicit confidence reports might capture certainty readouts more efficiently than explicit reports because, without a specific definition of confidence, it is not clear what subjects are actually reporting when they use explicitly-defined response scales (Persaud et al., 2007; Pouget et al., 2016). Under the assumption that implicit scales might be more reliable, one of the approaches, so called "post-decision wagering" (PDW), not only represents a more robust measurement of certainty readouts, but it also reflects simple computations employed when humans and other animals behave naturally.

During PDW, subjects bet resources (usually money for humans) on the accuracy, or correctness, of their decisions in order maximize their gains and minimize their losses (Persaud et al., 2007). In this way, this motivating and goal-direct certainty scale encourages subjects to assess the reliability of the evidence that is supporting their decisions by reading out multiple variables involved in such decisions (such as sensory evidence and the internal state of the body) without having to transform these readouts into subjectively interpretable language-based formulations. In my point of view, PDW fits perfectly to the study of cognitive processes (and underlying neural activity) performed under the assumption that the brain encodes information holistically in order to create actions. 


\section{Bi-directional certainty}

During my initial investigations of certainty-related processes and associated literature, I realized that, although information about being incorrect is as important as information about being correct (Yeung and Summerfield, 2012), confidence was typically associated with certainty of having done a correct decision (Fleming et al., 2012; Hebart et al., 2014; Kepecs et al., 2008; Kiani and Shadlen, 2009; Pouget et al., 2016), whereas certainty of having done an incorrect decision was majorly studied separately in the context of error detection and changes of mind (Charles et al., 2013; Murphy et al., 2015; Rabbitt, 1966; Resulaj et al., 2009). Nevertheless, I began to appreciate the fact, as Yeung and Summerfield (2012) also did, that confidence and error detection might be, in a sense, "two sides of the same coin". In my work, I named these processes as readouts of certainty of being correct and certainty of being incorrect in order to emphasize the integration of the two traditionally separate research fields. It did not make sense for me, for example, that these two processes were approached from the previous error detection binary (all-or-none) perspective (i.e. subjects report whether they believed their decision was incorrect or not; e.g. Charles et al., 2013). I hypothesized, instead, that both readouts are based on probabilistic assessments and could be reported using continuous (i.e. non-binary) scales.

While my colleagues and I were collecting data to test the hypothesis that both readout "directions" (certainty of being correct and certainty of being incorrect) can be measured using the same continuous scale, Boldt and Yeung (2015) published a study that corroborated my expectations and demonstrated, by still using an explicitly formulated response scale, that there is a continuum in the readout of these two types of certainty. In their response scale, subjects reported certainty readouts ranging from “certainly wrong" to "certainly correct". Although Boldt and Yeung (2015) were the first ones to describe certainty bi-directionality, their scale depended on humans fully capable to use their language comprehension skills, as well as on conscious transformations of certainty readouts into verbally-defined categories. Consequently, it was not possible for them to contribute directly to the understanding of certaintyrelated processes under the basic computational framework proposed by Kepecs and Mainen (2012). 
Nevertheless, Boldt and Yeung (2015) provides solid support, together with other very recent studies, for the idea that individuals not only accumulate evidence prospectively in order to make proper decisions (Ratcliff and McKoon, 2008), but also continue to accumulate evidence after their decisions (Kiani et al., 2014; Murphy et al, 2015; van den Berg et al., 2016; Yu et al., 2015). The demonstration of such post-decisional evidence accumulation was essential to bring together the two certainty directions because it can be argued that certainty of being incorrect intrinsically depends on some sort of extra evidence (otherwise individuals who identified incorrect decisions would not make an incorrect choice in the first place) and, as I will propose in Chapter 1, it is preceded by a post-decisional decrease in certainty of being correct.

In this context, Chapters 1 and $\mathbf{2}$ describe the two experiments in humans that $\mathrm{I}$ developed to capture implicit bi-directional certainty readouts using PDW and to test if there are brain areas which encode such bi-directional processing, respectively. In these chapters, I was able to demonstrate that during wagering, subjects implicitly reported the increase of certainty of being incorrect towards the lowest wager, and the increase of certainty of being correct towards the highest wager. Moreover, I showed that certainty of being incorrect, in particular, was a likely consequence of postdecisional evidence accumulation. I also demonstrated that multiple brain areas were able to encode these readouts, suggesting that PDW task demands and adaptive strategies (i.e. try to gain more when correct and avoid large losses when incorrect) indeed prompted brain areas to encode the information in the most adaptive way.

\section{The comparative study}

My initial doctorate project proposal focused on the neural basis of adaptive choices of rhesus monkeys contrasting physical and mental efforts. Specifically, I planned a series of experiments based on Hampton (2001), who proposed - using a kind of wagering (opt-out procedure) before the decisions - that rhesus monkeys know when they remember. My aim was to investigate if rhesus monkeys have the capacity for introspection and awareness, and to identify, using fMRI, which brain areas they employ during such metacognitive processes. 
Since the development of fMRI in awake and behaving non-human primates has been shown to be essential to link the knowledge about the human brain obtained using non-invasive imaging techniques and the vast knowledge provided by invasive electrophysiological studies in monkeys (e.g. Goense et al., 2010; Kagan et al., 2010; Oleksiak et al., 2010; Patel et al., 2015; Vanduffel et al., 2001); and because the brain structure of monkeys and their behavioral complexity are closely related to humans (Arcaro et al., 2011; Kiani and Shadlen, 2009; Mantini et al., 2011; Vanduffel et al., 2014), I also intended to perform the same fMRI experiments in humans in order to compare the two primate species directly.

My first experiment, however, was designed to address a very simple question while I was training the monkeys to perform more complex tasks: how does the interhemispheric processing of visuomotor information differs between rhesus monkeys and humans? Although early studies about the transfer of information between the hemispheres date from the beginning of the last century (Poffenberger, 1912), some paradigms involving interhemispheric communication and integration of information during simple visuomotor responses were never tested in monkeys. Specifically, I wanted to investigate interspecies differences using the paradigm created by Poffenberger (1912), which tests the time the brain takes to transfer visuomotor information between the hemispheres by comparing two manual response conditions: responses which do not require interhemispheric communication because visual representation and motor responses can be encoded in the same brain hemisphere (uncrossed responses); and responses which require interhemispheric communication because the visual information is first represented in the hemisphere that does not encode the motor response (crossed responses).

However, at the moment I realized that - due to problems with monkey training and delays in the production of magnetic resonance-compatible equipment specific for monkey experiments - I would not be able to perform the Poffenberger-related experiments with both primate species and successfully move to the experiments I initially planned to investigate introspection in monkeys; I decided to test how interhemispheric processes would be modified if I were to add different levels of certainty about the upcoming response requirements. I also decided to partially pursue my initial scientific questions by testing the capacity of introspection only in humans. 
As mentioned above, this investigation is described in the metacognition-related studies of Chapters 1 and 2.

Chapter 3, consequently, describes a comparative study conducted in parallel to the experiments about bi-directional certainty. In order to address interhemispheric communication under different certainty levels, and to test how the varying predictability about upcoming response modifies intra-and inter-hemispheric action selection processing, I used three tasks with different randomization of visual stimulus location and motor response. Therefore, instead of investigating the certainty associated with preceding decisions (as in PDW), I compared how the predictability determined by the trial history modifies the ensuing visuomotor processes. The interspecies comparisons were mainly based on differences regarding brain (a)symmetry and lateralization (for review see Oleksiak et al., 2010); brain size (for review see Aboitiz et al., 2003); and the capacity each species has to represent the abstract components of my tasks.

In summary, the present thesis aimed at investigating neurobiological aspects of two ubiquitous processes in the nervous system: decision-making and certainty readouts. I based my investigation on the assumption that the brain encodes information in order to adapt animals' behaviors to the complex environment by facilitating flexible action selection. I did not address evolutionary causes (selective processes) for the investigated decision-making processes directly, but I took into account the consequences of anatomical and functional differences between monkeys and humans to their behavior. 


\section{Chapter 1}

\section{Certainty bi-directionality}

\section{and the efficient use of metacognition}

(content of this chapter has been submitted to Cognition as: Moreira, C. M., Rollwage, M.,

Kaduk, K., Wilke, M., Kagan, I. Certainty bi-directionality and the efficient use of metacognition) 


\section{Table of Contents}

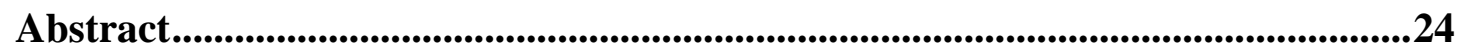

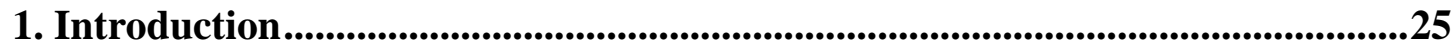

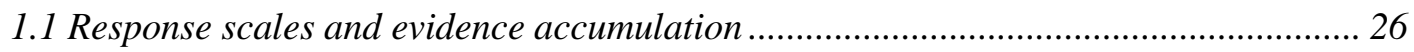

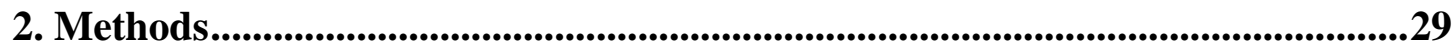

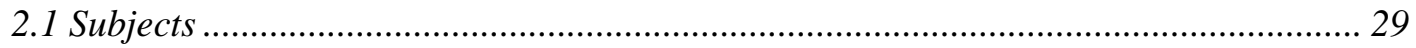

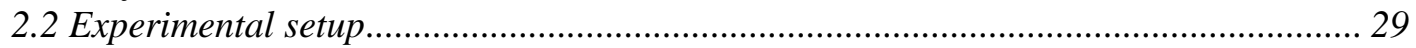

2.3 Type 1 task: delayed match-to-sample task................................................................. 30

2.4 Pre-decision wagering, post-decision wagering and the control task .......................... 30

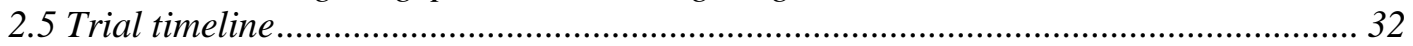

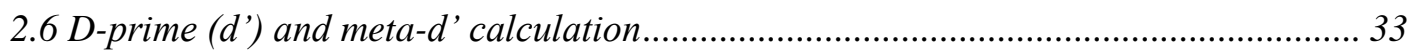

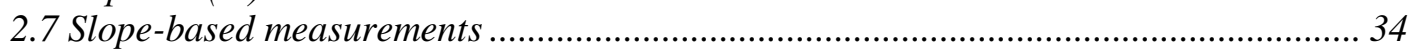

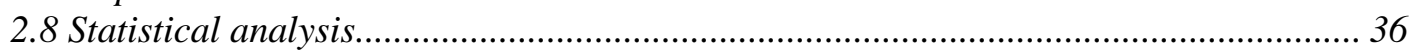

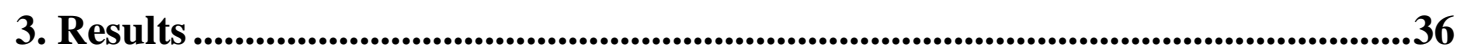

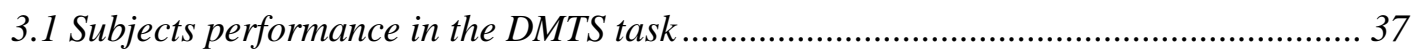

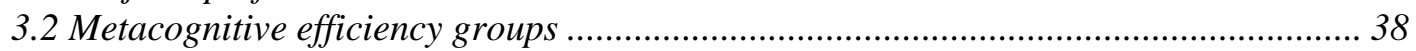

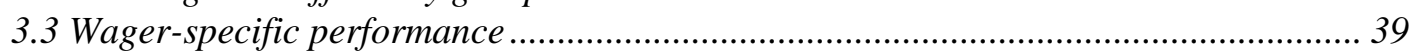

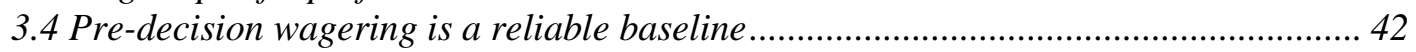

3.5 Readouts varied between the metacognitive efficiency groups .................................... 43

3.6 Slope-based metacognitive ability is compatible with meta-d' ..................................... 44

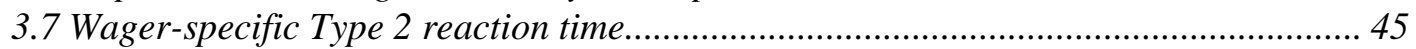

3.8 Slope-based measurements for each difficulty level ................................................... 46

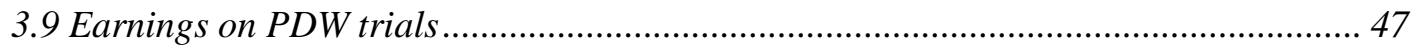

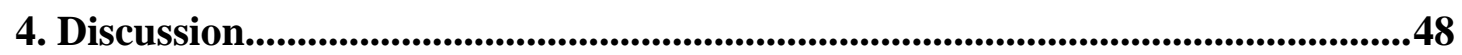

4.1 Metacognitive efficiency as a measure of evidence accumulation................................ 49

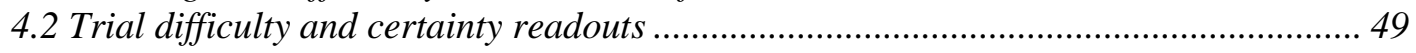

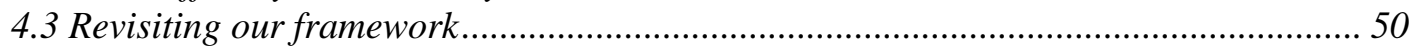

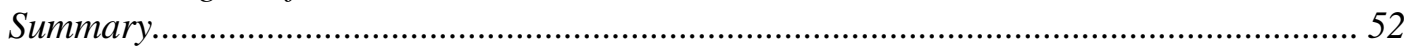

Supplementary material ..................................................................................54

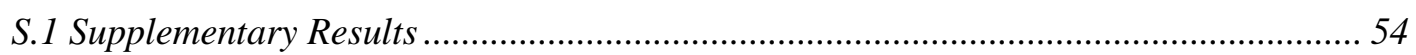

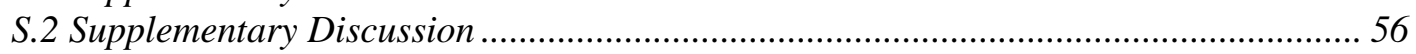

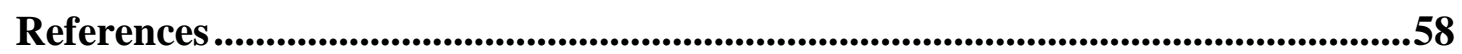




\begin{abstract}
Humans and other animals constantly evaluate their decisions in order to learn and behave adaptively. Experimentally, such evaluation processes are accessed using metacognitive reports. When made after decisions, metacognitive reports might reflect not only different levels of decision certainty, but also two certainty directions (certainty of being correct and certainty of being incorrect). It is important to test if such bi-directional processing can be measured because, for adaptive decisionmaking, information about being incorrect is as important as information about being correct. We were able to capture bi-directional certainty readouts by asking subjects to bet money on their perceptual decision accuracy using a six-grade wager scale (post-decision wagering, PDW). To isolate trial-specific aspects of metacognitive judgments, we used pre-decision wagering trials (wagering before the perceptual decision) to subtract, from PDW trials, wagering influences resulting from non-trialspecific assessment of expected difficulty and psychological biases. This novel measure of metacognitive ability allowed independent quantification of certainty of being correct and certainty of being incorrect readouts. Bi-directional certainty readouts were associated with increased sensitivity during wagering in comparison to perceptual decisions (i.e. high metacognitive efficiency), suggesting a link between post-decisional evidence accumulation and metacognitive efficiency. We also show that both readouts increased on easier trials, demonstrating that certainty of being incorrect is not confounded with low certainty. The quantification of bi-directional certainty by the same implicit graded response scale suggests that both readouts represent similar, or even the same, metacognitive process.
\end{abstract}

Key-words: certainty, metacognition, metacognitive efficiency, post-decision wagering, post-decisional evidence, confidence

Abbreviations: pre-decision wagering (PreDW), post-decision wagering (PDW), delayed match-to-sample task (DMST) 


\section{Introduction}

Humans and other animals are able to assess their own cognitive processes (perception, memory and decisions) to flexibly adapt their behavior (Fleming and Lau, 2014; Hampton, 2009; Kepecs and Mainen, 2012). This assessment (i.e. metacognition) can be understood as a resulting readout of the certainty associated with cognitive processes (Kepecs and Mainen, 2012; Kepecs, 2013; Kiani et al., 2014; Ma and Jazayeri, 2014) and is especially useful to plan future actions more efficiently after decisions (Fleming et al., 2012a; Kepecs and Mainen, 2012; Kiani et al., 2014; Pouget et al., 2016).

The planning of future actions that relies on the evaluation of previous decisions might vary not only with certainty level (from low to high) but also with what we call “certainty direction" (i.e. certainty of being correct or certainty of being incorrect). For example, a person in a hurry goes to the supermarket and decides to enter an aisle to search for a specific product. If she is uncertain about her decision, she will probably slow down her pace and search for the product from a distance, staying close to other aisle options. This is not the best way to find a product, but it is the best way to avoid spending her main resource (in this case, time) on this uncertain decision. If she is highly certain about her decision, her planning of a next action might have two distinct outcomes depending on her certainty direction. If she is certain she chose the correct aisle (certainty of being correct), she will walk down this aisle to search for the product closely. On the other hand, if she is certain she made an incorrect decision (certainty of being correct), she will turn around and walk to another aisle.

Choice certainty, or confidence, has been extensively investigated in different contexts of perceptual, memory and value-based decision-making (e.g. Fleming and Dolan, 2012a; Hampton, 2001; Kiani and Shadlen, 2009; Monosov and Hikosaka, 2013), however the ability to implicitly use certainty of being correct and certainty of being incorrect was never measured in the same experiment. Therefore, the main goals of the present study were to capture bi-directional readouts of certainty without relying on explicit verbal formulations, and to relate those readouts with other cognitive processes (e.g. post-decisional evidence accumulation). As a first step, we consider previous assumptions about the relationship between perceptual evidence and confidence judgments in order to create a framework that supports such bidirectional readouts. 


\subsection{Response scales and evidence accumulation}

In Signal Detection Theory (SDT), what has been referred so far as "decisions" are defined as "Type 1 decisions"; while decisions about correctness of Type 1 decisions are named "Type 2 decisions" (Clarke et al., 1959; Galvin et al., 2003). Type 2 decisions, in the form of confidence ratings, are often applied to measure how certain subjects are about having done a correct decision (e.g. Fleming and Lau, 2014; Hebart et al., 2014; Heereman et al., 2015; Maniscalco and Lau, 2012; Sandberg et al., 2010). In such unidirectional formulations, reports of low confidence are thought to signify low certainty, while certainty of being incorrect is explicitly not considered.

In some cases, the experimenters exclude the presence of high certainty of being incorrect by instructing subjects to abort trials when they "know" they have done incorrect choices (e.g. Hebart et al., 2014). But more commonly, it is assumed that the same evidence used for Type 1 decisions is also used for Type 2 decisions (e.g. Charles et al., 2013; Galvin et al., 2003; Maniscalco and Lau, 2012), thus precluding the realization of errors. Challenging this assumption, recent studies highlighted the possibility of post-decisional evidence accumulation, building on a combination of signal detection and drift diffusion models to investigate the accumulation of evidence after Type 1 decisions (e.g. two-stage Dynamic Signal Detection model of Pleskac and Busemeyer, 2010; Moran et al., 2015; Yu et al., 2015). Further support for postdecisional evidence accumulation has been provided by studies investigating changes of mind and error detection (Kiani et al., 2014; Murphy et al, 2015; Yeung and Summerfield, 2012; van den Berg et al., 2016), and this process has been associated with both certainty directions (Boldt and Yeung, 2015).

In order to explore this association, we illustrate three different scenarios based on different assumptions about evidence accumulation and different interpretations of the certainty scale (Fig. 1). These scenarios are exemplified using a task similar to the one used in our experiment: a delayed match-to-sample task (DMTS, Type 1 decision) followed by a six-grade certainty scale (Type 2 decision). The sample is presented for a certain period and, afterwards, subjects should respond if the match is on the right or on the left side of the screen. When the two match possibilities are presented, subjects start accumulating evidence towards both of them and, since response time is limited, at certain point they commit to the Type 1 decision by reading out if the difference between the evidence supporting each option (i.e. decision variable) lies on the right 
or on the left side of the Type 1 criterion (i.e. subjective criterion used to classify the evidence). Following the DMTS task, subjects are asked to report their certainty in the preceding choice. Generally, it is assumed that subjects assess certainty by reading out the distance between the evidence and the Type 1 criterion (Gold and Shadlen, 2001; Herbart et al., 2014; Kiani et al., 2014).

In the illustrated example, the subject makes an incorrect Type 1 decision. The first scenario represents the model without post-decisional evidence accumulation (Fig. 1A). In the example, since the evidence at (and after) the Type 1 decision is close to the Type 1 criterion, the subject would rate her certainty as low (e.g. rating 2). Since scenario A has been challenged by studies which show that subjects acquire additional evidence after Type 1 decisions (Murphy et al, 2015; Yu et al., 2015), scenario B exemplifies how confidence reports might be misinterpreted if this postdecisional evidence is not considered for the formulation and interpretation of the certainty scale. In scenario B, the drift after the Type 1 decision could lead the evidence even further right from the Type 1 criterion or, as exemplified, to the other side of the criterion. In this example, the subject is more certain during the Type 2 decision (greater distance between the evidence and the Type 1 criterion) compared to the moment of the Type 1 decision. And, by taking into account the reported Type 1 decision, she could classify her certainty as certainty of being incorrect. However, due to typical unidirectional formulation of the certainty scale (e.g. Fleming et al., 2012b), certainty of being incorrect is conflated with low certainty of being correct (Fig. 1B).

We propose that scenario $\mathrm{C}$ is more appropriate if subjects accumulate post-decisional evidence because it affords the possibility to use the certainty scale to report both certainty of being correct and certainty of being incorrect. The difference between scenarios $\mathrm{B}$ and $\mathrm{C}$ is, therefore, the formulation and interpretation of the certainty scale. In scenario $\mathrm{C}$, certainty increases in both directions as, for example, a U-shape function. One half of the scale reflects the readout of certainty of being correct, and the other half reflects the readout of certainty of being incorrect (Fig. 1C). Such bidirectional scale might be achieved, for example, by suitable phrasing (e.g. certainty ranging from "certainly wrong" to "certainly correct", as in Boldt and Yeung, 2015) or by using monetary incentive formulations such as post-decision wagering (Persaud et al., 2007; Sandberg et al., 2010), the approach we adopted in this study. 


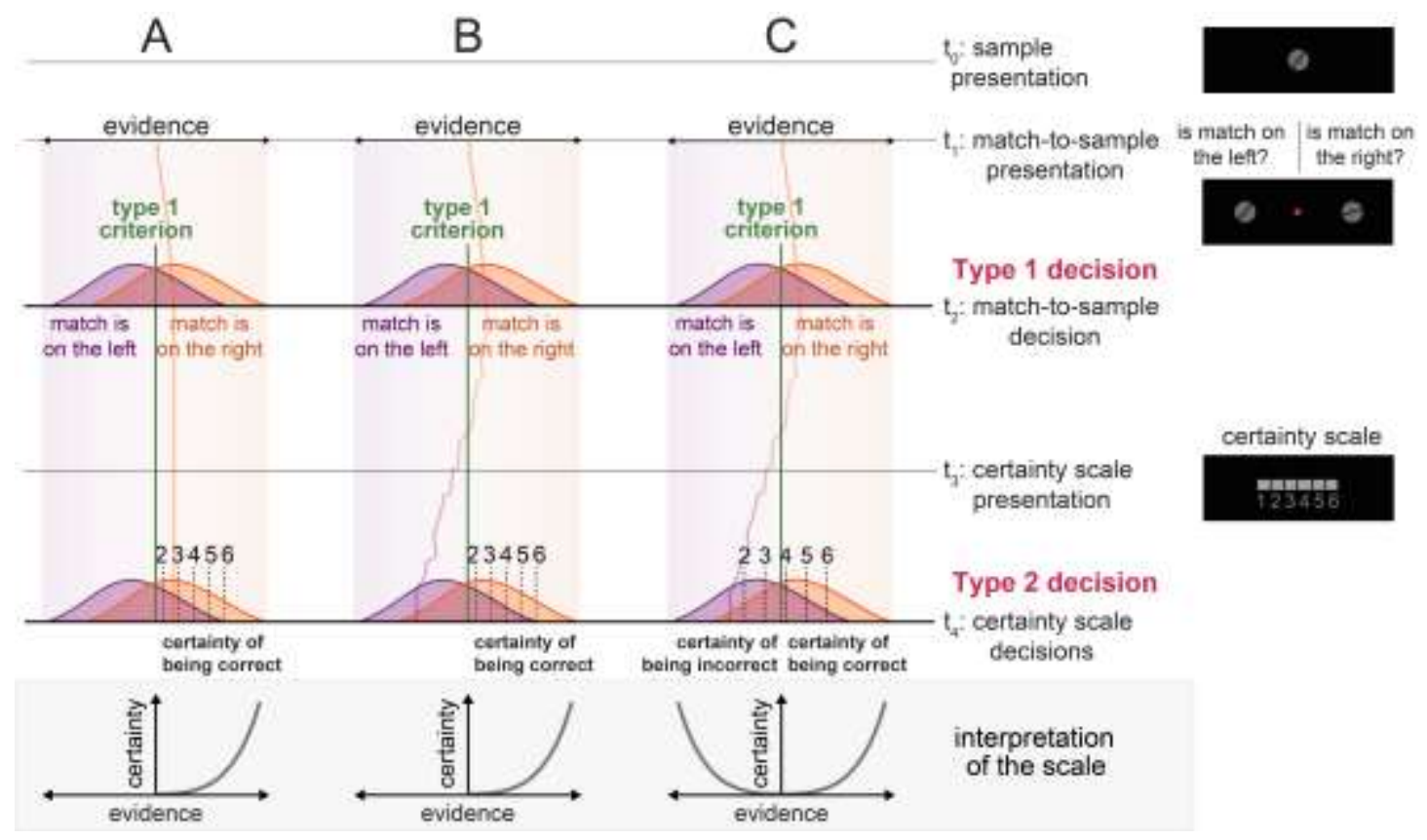

Figure 1. Three scenarios for evidence accumulation during one (incorrect) trial of a perceptual decision (match-to-sample, Type 1 decision) followed by certainty rating (Type 2 decision). The match was on the left, but the subject reported that the match was on the right. Trial time goes from top to bottom $\left(t_{0}\right.$ to $\left.t_{4}\right)$. (A) Scenario A represents the model without post-decisional evidence accumulation. The separation between the probability distribution functions of perceptual evidence (purple and orange curves) and their variance reflects subject's Type 1 sensitivity (d' in SDT) across all trials. Since the evidence is close to the criterion subject used to decide if the match was on the right or on the left side (Type 1 criterion), she should read out her certainty as low (in this example, the certainty was 2 because the evidence fell between her Type 2 criteria 2 and 3). (B) The scenario B assumes evidence accumulation after Type 1 decisions, but its consequences are not considered in formulation and interpretation of the certainty scale. After reporting that the match was on the right side of the screen, the subject kept accumulating evidence. In this example, the accumulated evidence crossed Type 1 criterion and indicated that the subject did an incorrect Type 1 decision. For Type 1 decisions to right, the scale formulation places Type 2 criteria along the evidence axis only to the right side of the Type 1 criterion, neglecting certainty of being incorrect. In this way it is not possible to dissociate if the subject was reporting low certainty of being correct or some level of certainty of being incorrect. (C) The scenario $\mathrm{C}$ allows post-decisional evidence accumulation and considers certainty bi-directionality when interpreting certainty ratings. In this way, it distinguishes levels of certainty of being correct from levels of certainty of being incorrect. It is important to emphasize that, in all scenarios, Type 2 criteria distribution would be flipped horizontally over the evidence axis in the case of Type 1 selection of the left option.

When post-decisional evidence accumulation occurs, subjects might improve their performance on Type 2 decisions compared to Type 1 decisions. Maniscalco and Lau 
(2012) developed a function to calculate Type 1 and Type 2 sensitivities on the same scale, allowing direct comparison between them. Since Maniscalco and Lau (2012) assumed that Type 1 and Type 2 decisions are based on the same evidence, they expect that Type 2 sensitivity (meta-d') should never exceed the Type 1 sensitivity (d'). In other words, the ratio meta-d'/d', termed as metacognitive efficiency, should not be higher than 1. We, on the other hand, suggest that values of meta-d' higher than d' are likely because subjects can accumulate evidence after the Type 1 decision, improving their Type 2 sensitivity.

In the present study we confirmed the assumptions proposed in the scenario $\mathrm{C}$ (increased certainty in both directions of the certainty scale as a likely consequence of post-decisional evidence accumulation) by capturing trial-specific readouts of certainty of being incorrect and certainty of being correct; and we associated postdecisional evidence accumulation with metacognitive efficiency.

\section{Methods}

\subsection{Subjects}

Eighteen subjects (6 males; mean age 25.7 years) were recruited via an online platform of the University of Goettingen. All subjects had normal or corrected-tonormal vision. One subject was discarded because he did not have sufficient number of trials in some of the conditions. Subjects were paid according to their performance (please see below). The experimental procedures were approved by the local ethics committee.

\subsection{Experimental setup}

Subjects sat in front of an LED screen (1600 x 1200 resolution) at $51 \mathrm{~cm}$ viewing distance and responded manually using two capacitive proximity sensors (buttons) connected to the computer via parallel port. Subjects positioned their head over an adjustable chin rest and had their head fixed with an adjustable strap for better stabilization. Gaze position was acquired with $60 \mathrm{~Hz}$ miniature infrared eye tracker camera and ViewPoint 2.8.6.21 software (Arrington Research). The task was controlled via MATLAB (Mathworks Inc) using the Psychophysics toolbox 
(http://psychtoolbox.org/). Subjects performed practice trials until they became familiar with this experimental setup.

\subsection{Type 1 task: delayed match-to-sample task}

Subjects completed 360 trials of a visual delayed match-to-sample (DMTS, Type 1 decision) task in which they had to find, between two options, the match for a preceding sample, which consisted of one gray circle of $1.5^{\circ}$ of visual angle radius with an oblique black bar crossing its center (Fig. 2). Ten different sample options were generated by varying the bar orientation in counterclockwise rotation from the horizontal plane (from $18^{\circ}$ to $58.5^{\circ}$ ). One of these ten samples was presented pseudorandomly at the beginning of each trial in the center of the screen. During match-tosample presentation, one sample-like image was presented $9^{\circ}$ to the right and another one $9^{\circ}$ to the left of the center of the screen (eye fixation spot). One of them had a bar in the same orientation as the sample (match) and the other one had a bar in a different orientation (non-match). Subjects responded by using the button of the hand positioned in the same side of the screen of the image selected as the match (Fig. 2A). Five difficulty levels were created by different orientation contrasts between the match and the non-match (from $4.5^{\circ}$ to $22.5^{\circ}$; Fig. 2B). Trials with different difficulty levels were grouped into three families. The overall level of difficulty of each family was determined by the different proportions of trials of each difficulty level. The sample color - green, blue or red - cued these families: easy, medium or hard, respectively (Fig. 2C).

Subjects were informed before the experiment that colors were related to different levels of difficulty, but they were not told about the link between specific colors and difficulty of each family.

\subsection{Pre-decision wagering, post-decision wagering and the control task}

The Type 2 decision was a wagering task in which subjects were asked to bet money on the correctness of their perceptual (Type 1) decision. They won the wagered money for correct Type 1 decisions and lost it for incorrect Type 1 decisions. In half of the 360 trials, subjects wagered after the Type 1 decision (Post-decision wagering, PDW) and in the other half of the trials subjects wagered before the Type 1 decision 
(Pre-decision wagering, PreDW). PreDW and PDW trials were pseudo-randomly interleaved. PreDW was used as baseline condition in further analyses. During either wagering trials, subjects made the Type 2 decisions by selecting first to wager high (wager categories 4, 5 and 6) or low (wager categories 1, 2 and 3), and afterwards by selecting a specific wager category among low or high options. They selected a specific wager within $3 \mathrm{~s}$ by using the button of the hand positioned in the same side of the corresponding selected option.

On PDW trials, subjects performed a control task before the perceptual decision, at the same period they were wagering on PreDW trials, and vice-versa for PreDW trials. The control task, in which subjects had to select a cued (by blue color) response option, worked as an "instructed" wagering (Fig. 2) and did not influence subjects' earnings. It aimed to equalize, across PDW and PreDW trials, the cognitive effort due to intervening distraction (visual stimulation, object selection, and corresponding time interval).

Subjects started the experiment with 10 Euros and could earn up to 30 Euros according to their performance. They wagered on the correctness of every Type 1 decision using the following pay-off matrix, which was explained to them before the experiment:

\section{Table 1}

Wagering pay-off matrix

\begin{tabular}{|c|c|c|c|c|c|c|}
\hline $\begin{array}{c}\text { Type } 1 \\
\text { decision }\end{array}$ & \multicolumn{3}{|c|}{ Low wagers } & \multicolumn{3}{c|}{ High wagers } \\
\hline Correct & 2 cents & 5 cents & 8 cents & 11 cents & 14 cents & 17 cents \\
\hline Incorrect & -2 cents & -5 cents & -8 cents & -14 cents & -17 cents & -20 cents \\
\hline
\end{tabular}

As can be seen from the pay-off matrix, if subjects wagered low, they were rewarded and punished in the same way for correct and incorrect perceptual decisions. But when they wagered high, their incorrect perceptual decisions were punished with 3 cents more than they would be rewarded for correct Type 1 decisions. This pay-off matrix was designed during pilot experiments in which subjects reported that they knew they were performing generally above the chance level (50\%) and thus could earn money by simply wagering high all the time. To counteract this strategy, we 
encouraged subjects to evaluate every Type 1 decision by punishing high wagers associated to incorrect Type 1 decisions more than low wagers.

\subsection{Trial timeline}

Eye and hand movements were controlled throughout the trial. Each trial started with the appearance of a red sport and a gray framed-square in the center of the screen. Subjects were positioned in the rest position when they fixated the gaze inside the eye fixation window ( $3^{\circ}$ visual angle radius around the red spot) and, concomitantly, positioned the right and left thumbs over two separate buttons. After a variable delay $(0.5-1 \mathrm{~s})$ in the rest position, the sample was presented in the center of the screen for 1 s. After sample presentation, subjects had to maintain the rest position for another $1 \mathrm{~s}$ before the control task (for PDW trials) or the wagering task (for PreDW trials). Another period of 1s separated control/PreDW from the match-to-sample task (Type 1 decision). Subjects had up to $1.5 \mathrm{~s}$ to select the image they believed was the match. After the Type 1 decision report and another interval of $1 \mathrm{~s}$, subjects performed the wagering task (PDW trials) or the control task (PreDW trials, Fig. 2A).

A
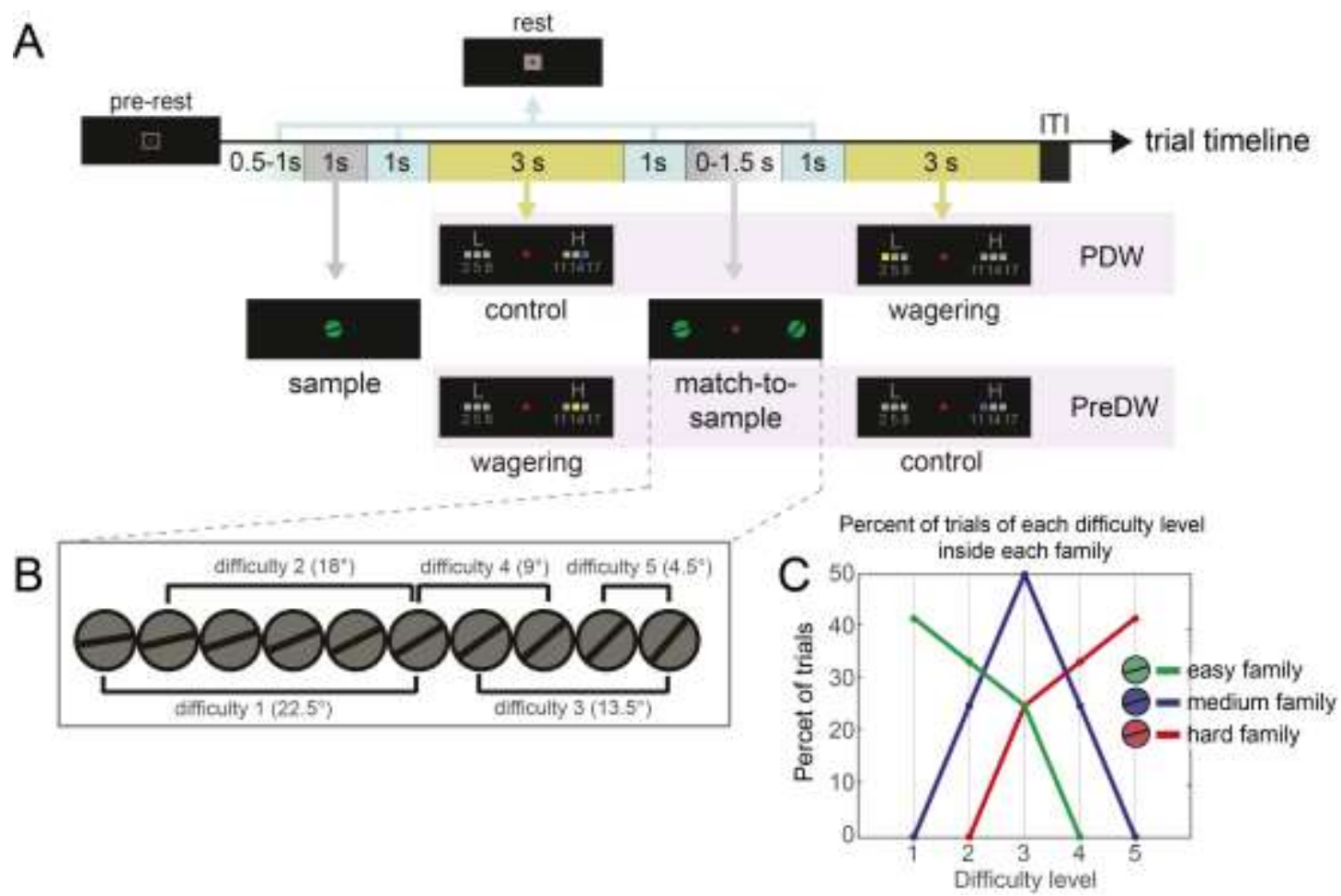

Figure 2. (A) Task design. The appearance of a red spot (for eye fixation) and of a gray framed-square (indicating buttons status) in the center of the screen signalized the trial start (pre-rest). The brightening 
of the red spot and the appearance of the gray filled square indicated that subjects correctly adopted the rest position. The sample was then presented in the center of the screen for $1 \mathrm{~s}$. During PDW trials, subjects performed first the control task. The letters H (high) and L (low) were presented each in one of the sides of the screen. The presentation side varied randomly. A blue square appeared above a specific wager and subjects had, first, to select high or low and then use the same button repeatedly to select the instructed wager option. The selection always moved from center-out. Overall, subjects had $3 \mathrm{~s}$ to select the instructed wager. Then, subjects performed the match-to-sample task by selecting the image they believed was the match. After that, subjects performed the wagering task, which was similar to the control task except that, after freely selecting high or low option, subjects could move the yellow square that appeared above one of the three wager options to select any desired option. PreDW trials were similar to PDW trials, with the difference that the wagering task and the control task order in the trial timeline were reversed. (B) Five difficulty levels were created by different orientation contrasts between the match and the non-match (linearly from $4.5^{\circ}$ to $22.5^{\circ}$ ). (C) Proportion of trials from the: easy family (green line), medium family (blue line) and hard family (red line) in each difficulty level (1 to 5).

There was no trial-by-trial feedback about the correctness of match selection (Type 1 decision). One feedback about the overall earnings was presented during a break that occurred after 180 complete trials, and the final earned value was presented after 360 complete trials. Trials in which subjects broke eye or hand fixation requirements, or were too slow to respond in one of task response periods, were aborted and repeated at a later time.

\subsection{D-prime (d') and meta-d' calculation}

In order to obtain a comparable and unbiased measure of Type 1 and Type 2 sensitivities, we used Maniscalco and Lau (2012) code provided at http://www.columbia.edu/ bsm2105/type2sdt/archive/index.html to calculate d' (Type 1 sensitivity) and meta-d' (Type 2 sensitivity). Meta-d' is calculated using the same parameters of a Type 1 SDT model, thus d' and meta-d' are in the same scale. The code estimates the value of $d$ ' that would have been required to produce the observed type 2 hits and false alarms. 


\subsection{Slope-based measurements}

We used the linear fits to the proportions of correct and incorrect Type 1 decisions each wager was assigned to, to assess the readouts of certainty of being correct and certainty of being incorrect, as explained below. We calculated these proportion by dividing the number of correct trials each wager was assigned to by the number of all correct trials (wager-specific proportion of correct trials) and, separately, dividing the number of incorrect trials each wager was assigned to by the number of all incorrect trials (wager proportion of incorrect trials). Next, we fitted a linear trend to wagerspecific proportions of correct trials and another linear trend to wager-specific proportions of incorrect trials. The slopes of those fits were named "slope-correct" and "slope-incorrect", respectively, and were associated to the ability to read out each certainty direction. For example, subjects whose proportion of correct trials increased towards the highest wager would demonstrate, through this positive slope-correct, the ability to read out certainty of being correct (see Fig. 3B).

However, a problem of using this approach to estimate how subjects read out certainties is that, in addition to trial-specific information, those wager-specific trial proportions might also be influenced by unspecific factors such as the general task difficulty and psychological biases (e.g. loss aversion). For example, subjects with higher loss aversion and/or facing harder Type 1 decisions might choose low wagers more often than high wagers. In this case, the resulting slope would not be zero, but negative, even without any assessment about the performance in specific trials. In order to disentangle the influences of such unspecific factors and the assessment of trial-specific performance, we used the PreDW task as the baseline for the slopebased measurements. During PreDW, subjects could only access their average performance in each family. The sample color - green, blue or red - indicated three families: easy, medium or hard, respectively. Subjects could learn that red-colored samples predicted, on average, harder trials; and they could develop some idea about the probability of each difficulty level in a specific family. But they were not able to predict, at the moment they were wagering, their actual trial performance. Consequently, subjects should assign wagers randomly to correct and incorrect trials, generating similar PreDW slope-correct and PreDW slope-incorrect values (Fig. 3A), the requirement for the baseline condition. 
During PDW, on the other hand, subjects should have access to their trial-specific Type 1 performance. The better they assess this information (i.e. metacognitive ability), the more PDW slope-correct and PDW slope-incorrect become distinct from each other and from baseline PreDW slopes. This happens when subjects are able to assign high wagers more often to correct trials and low wagers more often to incorrect trials. The difference between PDW slope-correct ( $\beta$ correct) and PreDW slopecorrect ( $\alpha$ correct) characterizes the readout of certainty of being correct. When subjects are able to detect correct trials, $\beta$ correct is larger than $\alpha$ correct (Fig 3B). The same calculation is done independently for incorrect Type 1 decisions, with inverted assumption: when subjects are able to detect incorrect Type 1 decisions, $\beta$ incorrect is smaller than $\alpha$ incorrect (Fig 3C).

Subjects' metacognitive ability (the ability in detecting correct and/or incorrect Type 1 decisions on a trial-by-trial basis) will be reflected in the sum of their abilities to read out certainty of being correct and certainty of being incorrect. The metacognitive ability reaches highest levels when subjects are able to read out both certainty directions (Fig. 3D).

Wagering when subjects:

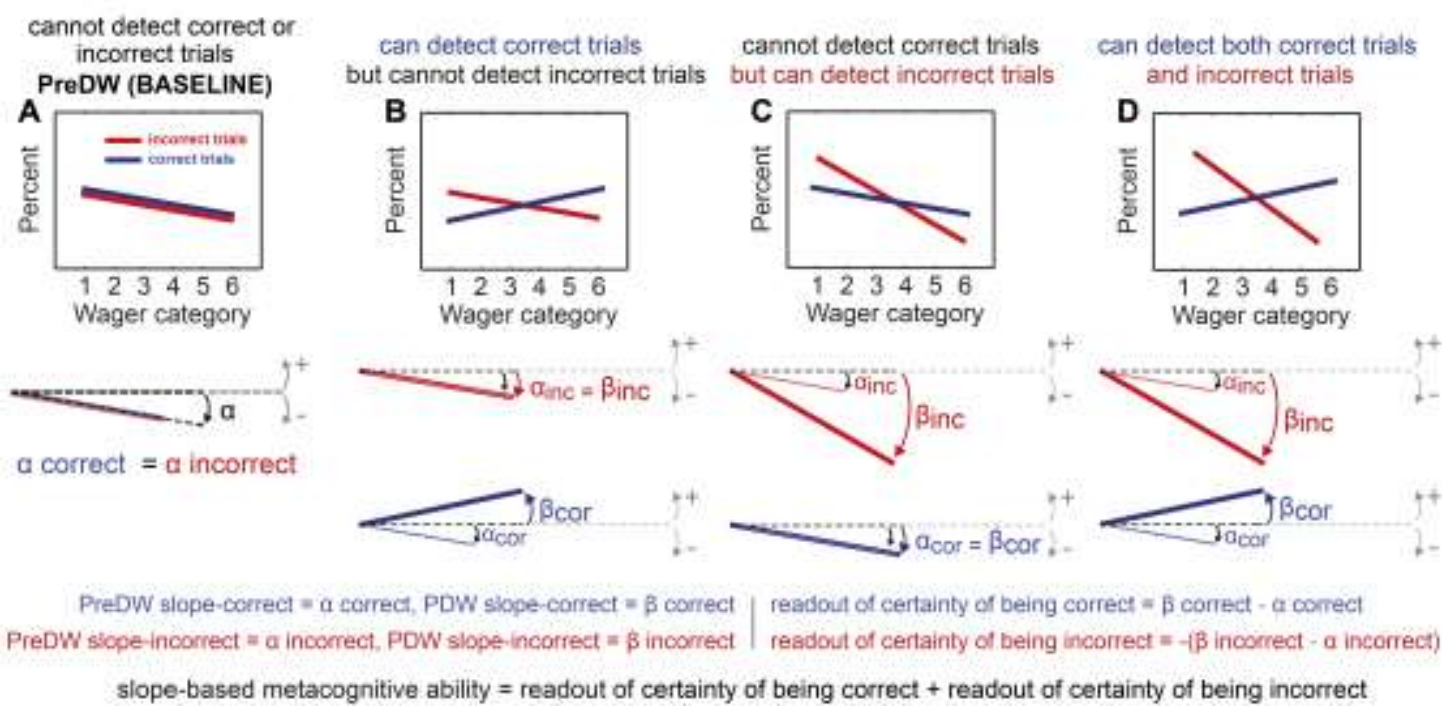

Figure 3. Framework to calculate readouts of certainty of being correct and certainty of being incorrect based on the slopes of linear fits to wager-specific proportions of correct and incorrect trials. (A) Slope-correct (angle between the blue linear fit and the horizontal plane) and slope-incorrect (angle between the red linear fit and the horizontal plane) when subjects cannot detect correct or incorrect trials (PreDW). Since subjects cannot tell from correct or incorrect trials, each wager has the same 
proportion of correct and incorrect trials and therefore the slopes should be the similar ( $\alpha$ correct $=\alpha$ incorrect). In this example, subjects use more often low wagers than high wagers, what leads to negative baseline slopes. (B) PDW slope-correct and PDW slope-incorrect when subjects can only detect correct trials. PDW slope-correct is different from PreDW slope-correct, indicating the readout of certainty of being correct, which is measured by subtracting PreDW slope-correct ( $\alpha$ correct) from PDW slope-correct $(\beta$ correct). The readout of certainty of being incorrect is still at zero level ( $\alpha$ incorrect $=\beta$ incorrect). (C) PDW slope-correct and PDW slope-incorrect when subjects can only detect incorrect trials. PDW slope-incorrect ( $\alpha$ incorrect) is different from PreDW slope-incorrect ( $\alpha$ incorrect), indicating the readout of certainty of being incorrect. (D) PDW slope-correct and PreDW slope-incorrect when subjects can detect both correct and incorrect Type 1 decisions. $\beta$ correct and $\beta$ incorrect are different from $\alpha$ correct and $\alpha$ incorrect, respectively. In this case, slope-based metacognitive ability (the sum of both certainty readouts) is partially influenced by the readout of certainty of being correct and partially influenced by the readout of certainty of being incorrect.

It is important to emphasize that, although we use words "identify" and "detect", we believe that reading out certainty is a probabilistic process. Therefore the readouts reflect the detection of correct and incorrect Type 1 decisions in a probabilistic manner.

\subsection{Statistical analysis}

We performed one-way, two-way and mixed-effects ANOVAs, linear correlations or t-tests using MATLAB (Mathworks Inc), as specified in the Results. R (The R Foundation) was used to perform multiple regression and linear mixed-effects regression models ( $\mathrm{R}$ package nlme; Pinheiro et al., 2007). The mixed-effects ANOVAs and the linear mixed-effects regression models allowed us to include all data (unbalanced design) and still utilize repeated measures when appropriate. When required, post-hoc tests were performed and corrected using Bonferroni correction.

\section{Results}

17 human subjects were asked to carry out a visual perceptual decision of varying difficulty (delayed match-to-sample, DMTS task) and a wagering task either before (pre-decision wagering, PreDW) or after (post-decision wagering, PDW) the perceptual decisions. Trial types (PreDW and PDW) and difficulty levels were randomly interleaved. 


\subsection{Subjects performance in the DMTS task}

We performed a two-way ANOVA for repeated measures to assess if perceptual (Type 1) performance varied between trial types (PreDW and PDW, factor 1) and among difficulty levels (factor 2). As expected, subjects performed better in the DMTS task on trials of lower difficulty (mean \pm SE for difficulty levels 1 to 5 : 86.7 \pm 3.3 81.4 $\left.\pm 2.275 .0 \pm 3.067 .8 \pm 2.449 .7 \pm 2.7 \% ; \mathrm{F}_{4,64}=51.439, \mathrm{p}<0.0001\right)$. There was no difference in average Type 1 performance between PreDW and PDW trial types $\left(\mathrm{F}_{1,16}=2.104, \mathrm{p}=0.17\right)$ and no interaction effect $\left(\mathrm{F}_{4,64}=0.970, \mathrm{p}=0.43\right)$, showing that subjects performed similarly in PreDW and PDW trial types across five difficulty levels.

We next tested with separate linear mixed-effects regression models for PreDW and PDW trials if there were differences in wagering among the five difficulty levels or among the three families. As described in Methods, families had different proportions of trials of each difficulty level (Fig. 2C), and were signaled to subjects by the color of a sample. As expected, during PreDW trials subjects wagered differently according to the families ( $\mathrm{p}<0.001$ for all pair-wise comparisons between families), and not according to the actual trial difficulty level, which was unknown to the subjects at the moment they were wagering ( $>0.05$ for all difficulty levels; Fig. 4A). In PDW trials, although there was a significant difference between easy and hard families $(p<0.05)$, this difference was driven by the actual difficulty levels $(\mathrm{p}<0.05$ for the comparisons between all difficulty levels, except between difficulty levels 1 and 2; Fig. 4B).

These results show that during PreDW subjects understood the differences among the families and wagered according to them. The results also indicate that during PDW trials subjects did not rely solely on the sample color (which signaled the average difficulty of each family). Instead, they also used the information accessed through the direct comparison between the two match options (trial-specific difficulty level). 

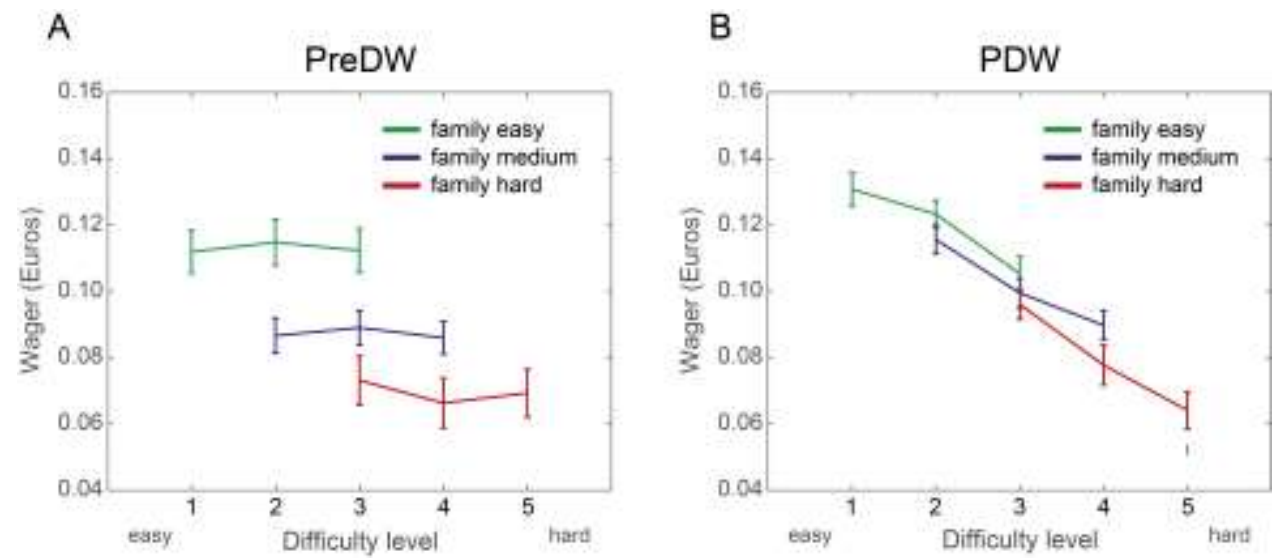

Figure 4. Means and standard errors of PreDW (A) or PDW (B) per difficulty level for each family: easy (green), medium (blue) and hard (red).

The following results will address only the PDW trials, except for the slope-based measurements which use PreDW trials as its baseline component.

\subsection{Metacognitive efficiency groups}

In the present experiment, d' (Type 1 sensitivity) reflects how well subjects identified the match during Type 1 decisions; and meta-d' (Type 2 sensitivity) reflects how well subjects used wagers to identify correct and incorrect Type 1 decisions. We used Maniscalco and Lau (2012) method to measure d' and meta-d' on the same scale and to compare them directly. We plotted meta-d' as a function of d', and distinguished between two groups of subjects: a group of 11 subjects with meta-d'>d' falling above the diagonal (high metacognitive efficiency group), and a group of 6 subjects with meta-d' $<$ d' falling below the diagonal (low metacognitive efficiency group; Fig. 5A).

Since we used a post-hoc grouping approach, it was important to check if the measurements (d' and meta-d') we used to create those groups varied significantly in the intergroup comparison. We applied a mixed-effect ANOVA with two factors: type of measurement (d' and meta-d', within-subjects) and group (meta-d'>d' and metad' $<$ d', between-subjects). Meta-d' was not different from d' across the entire sample $\left(\mathrm{F}_{1,15}=1.118, \mathrm{p}=0.31\right)$, and there was no group difference averaging the two measurements $\left(\mathrm{F}_{1,15}=4.256, \mathrm{p}=0.06\right)$. However, the interaction effect was significant $\left(F_{1,15}=24.995, p<0.001\right)$. Post-hoc tests revealed that the two groups had the same d' $\left(\mathrm{t}_{15}=0.051, \mathrm{p}=0.96\right)$, but the group of subjects with high metacognitive efficiency had 
higher meta-d' than the group of subjects with low metacognitive efficiency $\left(\mathrm{t}_{15}=3.198, \mathrm{p}<0.05\right.$; Fig. 5B). This result allowed us to compare the two groups, knowing that intergroup differences were not associated with differences in subjects' Type 1 sensitivity.
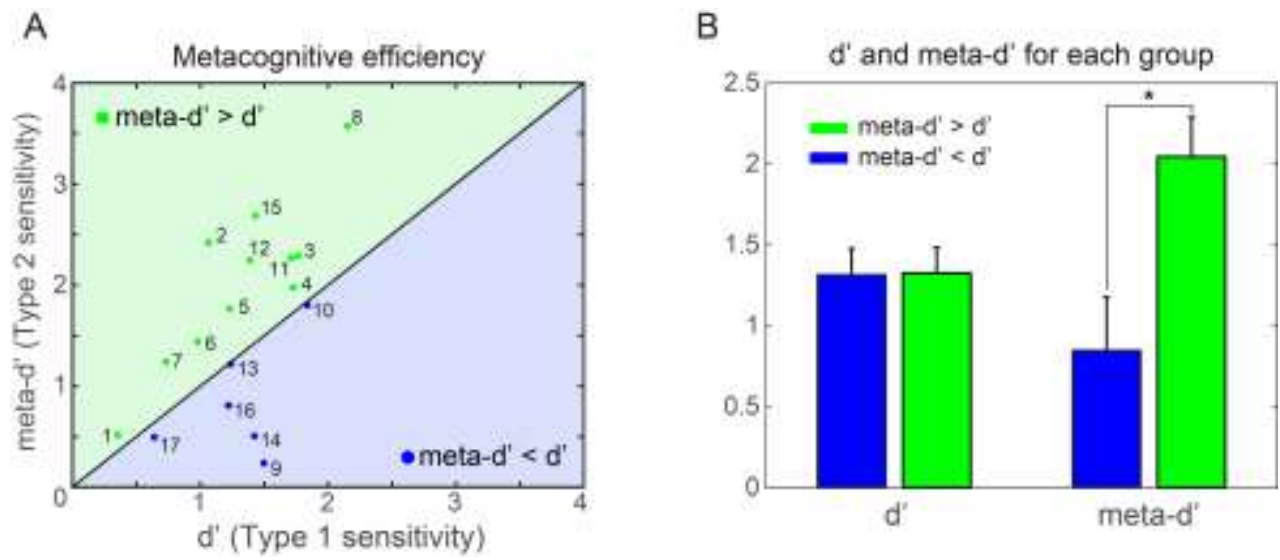

Figure 5. (A) Meta-d' plotted as a function of d'. 11 subjects with meta-d'>d' (high metacognitive efficiency group) fell above the equality diagonal (green area), and 6 subjects with meta-d' $<\mathrm{d}$ ' (low metacognitive efficiency group) fell below the diagonal (blue area). (B) Means and standard errors of d' and meta-d' values for each group: meta-d' $>d$ ' (green bars) and meta-d' $<$ d' (blue bars; * $p<0.05$ ).

\subsection{Wager-specific performance}

Next, we analyzed Type 1 performance per wager category using mixed-effects ANOVA with factors: performance per wager (within-subjects) and groups (betweensubjects). Subjects performed better on trials they selected higher wagers $\left(\mathrm{F}_{4,60}=23.837, \mathrm{p}<0.0001\right)$. In accordance with the results based on Type 1 and Type 2 sensitivities, the interaction effect showed that, although the high metacognitive efficiency and low metacognitive efficiency groups had the same general Type 1 performance $\left(\mathrm{F}_{1,15}=0.107, \mathrm{p}=0.75\right)$, wager-specific Type 1 performances were different between the groups $\left(\mathrm{F}_{4,60}=7.077, \mathrm{p}<0.0001\right.$; Fig. $\left.6 \mathrm{~A}\right)$. While the meta-d' $<\mathrm{d}$ ' group Type 1 performance varied from $58.7 \%$ in the lowest wager to $82.1 \%$ in the highest wager, the meta-d'>d' group had a range varying from below chance performance in the lowest wager $(43.1 \%)$ to $94.7 \%$ in the highest wager. Since the general Type 1 performance did not vary between the groups, the difference in the range of wager-specific Type 1 performance can only be explained by different Type 2 sensitivities. 
Furthermore, the below chance performance in the wager category 1 for the meta$\mathrm{d}^{\prime}>\mathrm{d}$ ' group $\left(43.1 \%, \mathrm{t}_{10}=-2.569, \mathrm{p}<0.05\right)$ is a strong indicator that subjects with high metacognitive efficiency were reading out certainty of being incorrect to detect incorrect Type 1 decisions and to assign them to the lowest wager.

To understand if there was a continuum in the relationship between wager-specific Type 1 performance and metacognitive efficiency, instead of discrete group differences, and to assess how much each wager-specific Type 1 performance explains inter-subject differences in metacognitive efficiency, we calculated a correlation between each wager-specific Type 1 performance and metacognitive efficiency, across subjects. We found that Type 1 performance in the wager categories 1, 2, 5 and 6 correlated significantly with metacognitive efficiency, and that absolute correlation values increased towards extreme wagers, generating a U-shaped curve (Fig. 6B, black symbols). The significant correlations for high and low wagers had opposite signs. Type 1 performance for the wager categories 5 and 6 correlated positively with metacognitive efficiency. This correlation indicates that subjects with higher metacognitive efficiency assigned more high wagers to correct trials. For the wagers 1 and 2 the correlations were negative, indicating that subjects with higher metacognitive efficiency were those who assigned more low wagers to incorrect trials.

Although the Type 1 performance in the wager categories 2, 5 and 6 helped to explain inter-subject variation in metacognitive efficiency, the strongest correlation occurred for the lowest wager $(R=-0.8, p<0.0001$; Fig. $6 C)$. As shown in the Figure $6 \mathrm{C}$, the Type 1 performance in the wager category 1 was below the chance level (50\%) for several subjects with high metacognitive efficiency, which we view as a clear demonstration of the readout of certainty of being incorrect. Conversely, none of the subjects with low metacognitive efficiency showed below chance Type 1 performance for this wager.

Finally, since the accumulation of evidence after the Type 1 decision might be essential for trial-specific detection of incorrect Type 1 decisions, the negative correlation between metacognitive efficiency and Type 1 performance in the wager category 1 might also suggests that subjects with higher metacognitive efficiency were those who accumulated more information after the Type 1 decision. 

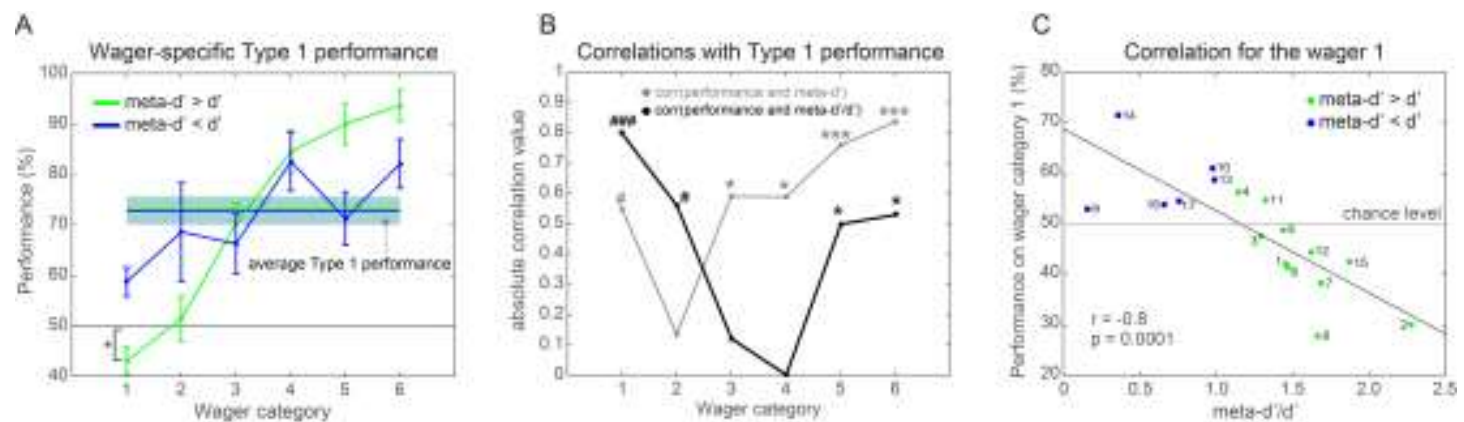

Figure 6. (A) Means and standard errors of Type 1 performance for each group (meta-d'>d': green line and band; meta-d' $<$ '': blue line and band). Type 1 performance for the meta-d' $>$ d' group (green curve) is below chance level in the lowest wager $\left({ }^{*} p<0.05\right)$. The horizontal black line represents the chance level (50\%) for the DMTS task. (B) Absolute values of correlation between wager-specific Type 1 decisions performance and metacognitive efficiency (black curve and symbols) and wager-specific Type 1 decisions performance and meta-d' (gray curve and symbols; *** $<0.001$ and ${ }^{*} \mathrm{p}<0.05$ for positive correlations, \#p<0.05 and \#\#\#p<0.001 for negative correlations). (C) Correlation between Type 1 performance in wager category 1 and metacognitive efficiency across subjects (subjects from the group meta-d' $>$ d' are in green and subjects from the group meta-d' $<$ d' are in blue). The horizontal dashed line represents the chance level for the DMTS task.

The pattern of correlations between wager-specific Type 1 performance and meta-d' was different from the one found for the ratio meta-d'/d'. The inter-subject differences in meta-d' presented stronger correlations with Type 1 performance in high wagers (Fig. 6B, gray symbols). This result indicates that meta-d' depended more on subjects' ability to detect correct Type 1 decisions and assign high wagers to them, while the metacognitive efficiency depended more on how subjects read out certainty of being incorrect to detect incorrect Type 1 decisions and assign low wagers to them.

In the next section we present further evidence for differences in the readout of certainty of being correct and certainty of being incorrect during PDW, and their relationship with subjects' metacognitive efficiency. The following results (sections 3.4-3.7) are based on measurements averaged across the five difficulty levels. In these measurements, we first averaged the results of different difficulty levels for one subject, and then we calculated averages across subjects. In the section 3.8 we present data on separate difficulty levels to establish their relationship with subjects' metacognitive readouts. 


\subsection{Pre-decision wagering is a reliable baseline}

As described in the Methods, slope-correct and slope-incorrect are independent measures that quantify readouts of certainty of being correct and certainty of being incorrect, respectively. These slopes are based on the linear trends fitted to the proportions of correct and incorrect trials each wager was assigned to. To isolate trialspecific information readouts, we created a baseline condition derived from PreDW trials. PreDW provided us with general wagering trends that subjects might have developed based on non-trial-specific information (such as expected difficulty and psychological biases). PreDW slopes were subtracted from PDW slopes since, on PDW trials, subjects had access to both: trial-specific performance and the same nontrial-specific information they had access during PreDW trials.

A reliable baseline should result in similar slope-correct and slope-incorrect values because, in the absence of trial-specific information, subjects were supposed to assign wagers randomly to correct and incorrect Type 1 decisions. We used two-way ANOVA for repeated measures to test if slope-correct is different from slopeincorrect (factor 1) depending on the trial type (PDW or PreDW, factor 2). Independently of the other factor, PreDW slopes did not differ from PDW slopes $\left(\mathrm{F}_{1,16}=0.007, \mathrm{p}=0.93\right)$ but slope-correct differed from slope-incorrect $\left(\mathrm{F}_{1,16}=64.039\right.$, $\mathrm{p}<0.0001)$. The interaction effect revealed that slope-correct and slope-incorrect were different depending on the trial type $\left(\mathrm{F}_{1,16}=42.493\right.$, $\left.\mathrm{p}<0.0001\right)$. Importantly, the posthoc test showed that this difference occurred during PDW trials $\left(t_{15}=9.433, p<0.01\right.$; Figs. 7B), but not during PreDW trials $\left(\mathrm{t}_{15}=2.204\right.$, $\mathrm{p}>0.05$, Fig. 7A), suggesting a reliable baseline.

We next estimated the readout of certainty of being correct by subtracting, for each subject, PreDW slope-correct from PDW slope-correct. The same was done to calculate the readout of certainty of being incorrect. The slope-based calculations demonstrated that subjects read out certainty in a bi-directional way (Fig. 7C). 

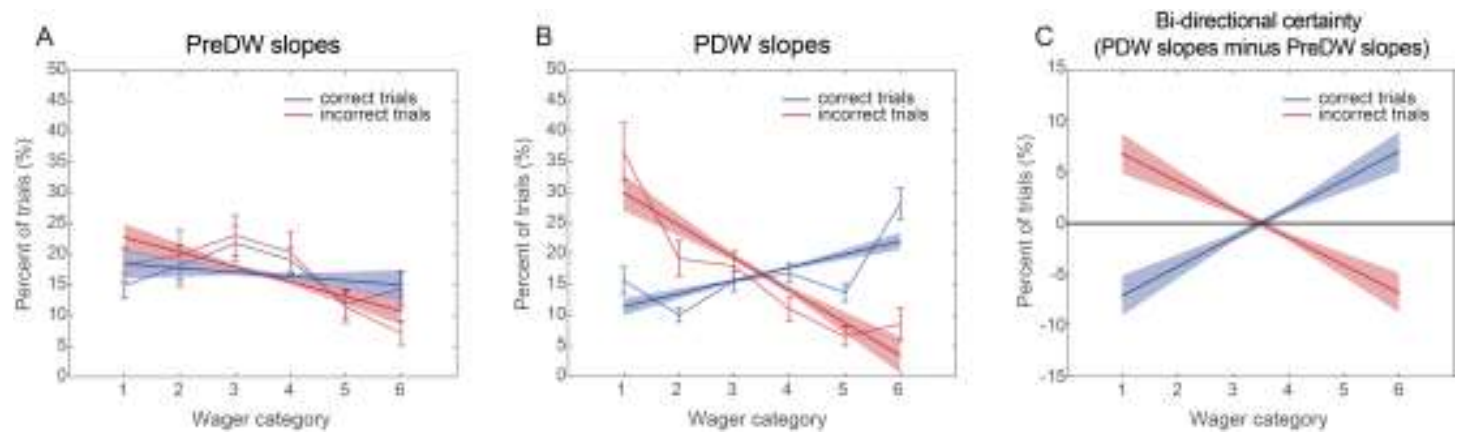

Figure 7. Means and standard errors of linear fits for correct trials (blue lines and shaded bands) and incorrect trials (red lines and shaded bands) for (A) PreDW (baseline) and (B) PDW, fitted to the data: means and standard errors of wager-specific proportion of correct (blue curves) and incorrect (red curves) trials. (C) Means and standard errors of PDW slope-correct minus PreDW slope-correct (blue line and band) and of PDW slope-incorrect minus PreDW slope-incorrect (red line and band). All measurements represent data across difficulty levels (averaged within each subject) and then averaged across subjects.

The next section addresses the group differences and the statistical differences between PreDW and PDW slopes.

\subsection{Readouts varied between the metacognitive efficiency groups}

We performed mixed-effect ANOVAs to test the differences not only between PDW and PreDW slopes for all subjects (within-subjects), but also between the two groups (meta-d' $>$ '' and meta-d' $<$ ', between-subjects). The first ANOVA revealed that slope-correct was higher for PDW compared to PreDW trials $\left(\mathrm{F}_{1,15}=10.218, \mathrm{p}<0.01\right)$. There was no group difference $\left(\mathrm{F}_{1,15}=0.017, \mathrm{p}=0.90\right.$; meta-d' $>\mathrm{d}^{\prime}: \mathrm{t}_{10}=2.791, \mathrm{p}<0.02$; meta-d'< $\left.<\mathrm{d}^{\prime}: \mathrm{t}_{4}=2.892, \mathrm{p}<0.05\right)$ or interaction between the factors $\left(\mathrm{F}_{1,15}=0.433, \mathrm{p}=0.52\right.$; Fig. 8A). The second ANOVA revealed that slope-incorrect was also different between PDW and PreDW for all subjects $\left(\mathrm{F}_{1,15}=11.070, \mathrm{p}<0.01\right)$ without group difference $\left(\mathrm{F}_{1,15}=1.825, \mathrm{p}=0.20\right)$. However, the interaction effect was significant $\left(\mathrm{F}_{1,15}=4.888, \mathrm{P}<0.05\right)$ and the post-hoc test revealed that PDW slope-incorrect was different from PreDW slope-incorrect only for the high metacognitive efficiency group $\left(\mathrm{t}_{10}=-4.742 ; \mathrm{p}<0.01\right.$; Fig. 8A). Figures $8 \mathrm{~B}$ and $8 \mathrm{C}$ further illustrate the difference between PWD and PreDW slopes for the two groups. Altogether, these results indicate that on average subjects read out both certainty directions during PDW. They also suggest that the difference of slope-based metacognitive ability (the 
sum of readouts of certainties of being correct and incorrect, Methods) between the groups ( $7 \pm 1$ for the meta-d' $>$ d' group and $3 \pm 1$ for the meta-d' $>$ d' group, non-paired ttest, $\left.\mathrm{t}_{15}=2.748, \mathrm{p}<0.05\right)$ was due to the ability of subjects with high metacognitive efficiency to read out certainty of being incorrect.
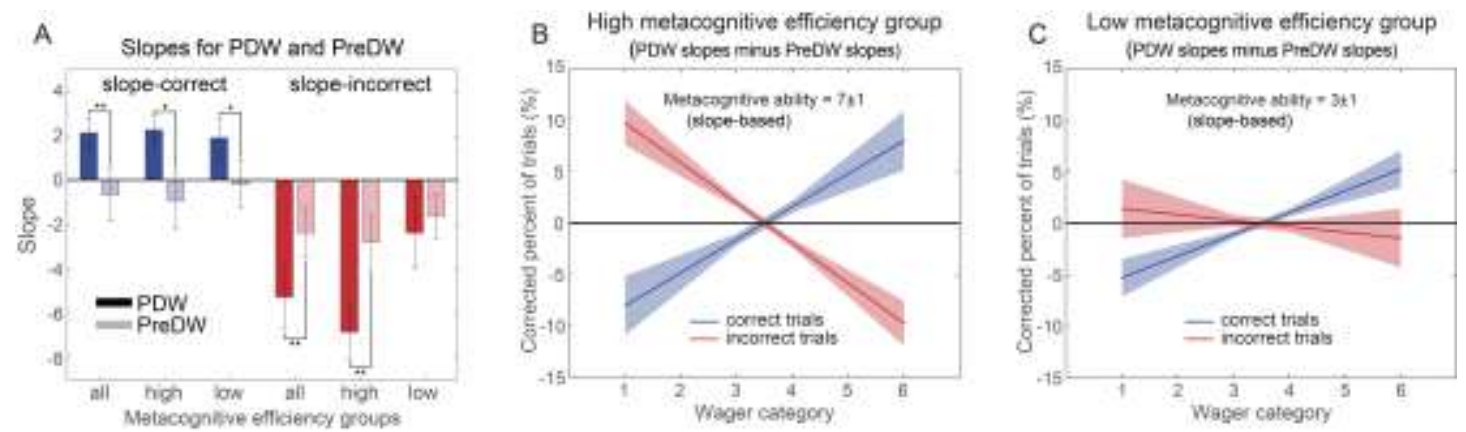

Figure 8. (A) Means and standard errors of PDW slope-correct (dark blue bars), PreDW slope-correct (light blue bars), PDW slope-incorrect (dark red bars), PreDW slope-incorrect (light red bars) from all subjects ("all"), high metacognitive efficiency group ("high") and low metacognitive efficiency group ("low"; *p $<0.05, * * \mathrm{p}<0.01$ for differences between PDW and PreDW). (B) Mean and standard error of PDW slope-correct minus PreDW slope-correct (blue line and shaded band) and of PDW slopeincorrect minus PreDW slope-incorrect (red line and shaded band), for high metacognitive efficiency group. The text in the top of the panel shows mean and standard error of slope-based metacognitive ability. (C) Same as B, but for the low metacognitive efficiency group. All measurements represent data across difficulty levels (averaged within each subject) and then averaged across subjects.

\subsection{Slope-based metacognitive ability is compatible with meta-d'}

Since slope-based measure is a new method we developed to assess metacognitive abilities, it is important to compare it with meta-d', a known and widely used measure. The strong positive correlation $(\mathrm{R}=0.89, \mathrm{p}<0.0001)$ between these two measures indicates that slope-based method can be considered a valid measure of metacognitive ability, with the advantage of allowing the independent quantification of certainty of being correct and certainty of being incorrect readouts (Fig. 9). 


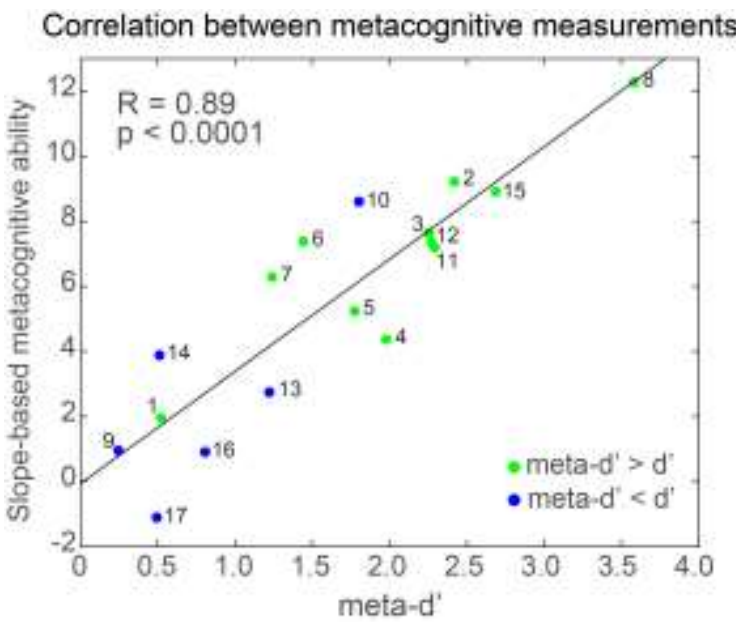

Figure 9. Correlation between two different measures of metacognitive ability. For each subject, the novel slope-based metacognitive ability measure is plotted against meta-d' value. The black line is the best linear fit between the two measurements. Subjects are colored according to the metacognitive efficiency group (green, high metacognitive efficiency, blue, low metacognitive efficiency), numbers indicate subject labels.

\subsection{Wager-specific Type 2 reaction time}

In the present experiment, each trial had two manual response periods. Type 1 reaction times reflect the time subjects took to report the perceptual decision (matchto-sample). Type 2 reaction times reflect the time subjects took to choose between high or low wagers. Type 1 and Type 2 reaction times were calculated separately for each wager (wager-specific RT1 and wager-specific RT2), to test the influence of Type 1 reaction times on subsequent wagering behavior, as well as the relationship between certainty and Type 2 reaction times.

The first mixed-effect ANOVA showed that mean wager-specific RT1 was unidirectional, decreasing when followed by high wagers $\left(\mathrm{F}_{5,75}=21.140, \mathrm{p}<0.0001\right)$. The interaction effect showed that subjects from the high metacognitive efficiency group responded faster during Type 1 decisions preceding some of the wagers $\left(\mathrm{F}_{5,75}=4.004, \mathrm{p}<0.01 ;\right.$ Fig. 10A $)$.

The second mixed-effect ANOVA showed that mean wager-specific RT2 differed among wagers $\left(\mathrm{F}_{5,75}=10.087, \mathrm{p}<0.0001\right)$. They were shorter in the wager categories 1 and 6 than the middle wagers (categories 3 and 4, p<0.05), generating an inverted Ushape function of wager-specific RT2 (Fig. 10B). Since faster reaction times are associated with increased certainty (Kiani et al., 2014), this result further supports our 
hypothesis that certainty increases in both directions of the wager scale. We associate fast Type 2 reaction times in the wager category 1 with increased certainty of being incorrect and fast Type 2 reaction times in the wager category 6 with increased certainty of being correct. The group comparison with mixed-effect ANOVA showed there were no significant differences between the groups $\left(\mathrm{F}_{1,15}=0.397, \mathrm{p}=0.54\right.$; Fig. 10B).
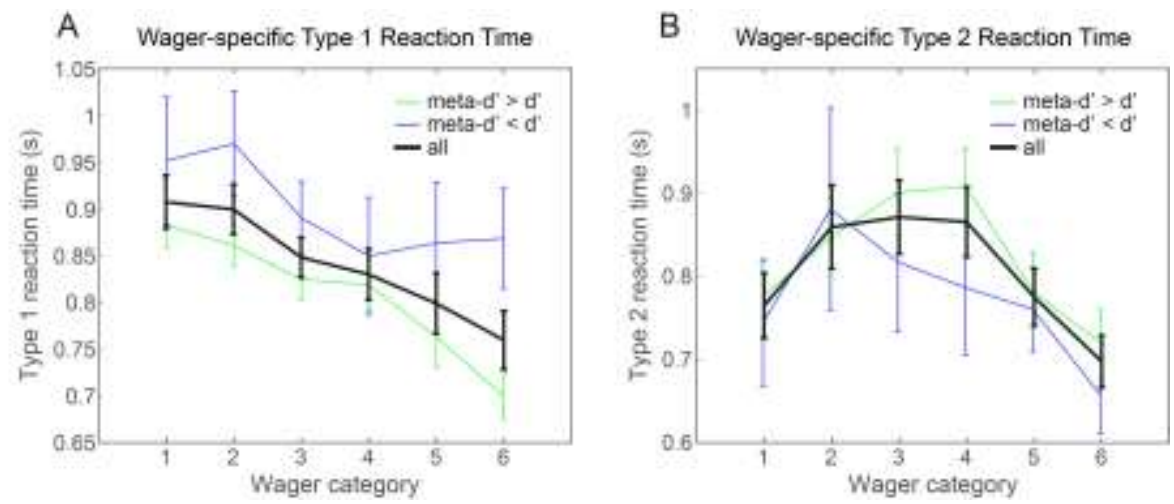

Figure 10. (A) Means and standard errors of wager-specific Type 1 (perceptual decision) reaction times (RT1) across all subjects (black curve), for the high metacognitive efficiency group (green curve) and for the low metacognitive efficiency group (blue curve). (B) Means and standard errors of wagerspecific Type 2 reaction times (RT2) across all subjects (black curve), for the high metacognitive efficiency group (green curve) and for the low metacognitive efficiency group (blue curve). All measurements represent averages across difficulty levels and across subjects.

\subsection{Slope-based measurements for each difficulty level}

We used Maniscalco and Lau (2012) approach to calculate the Type 1 decision criterion separately for the different difficulty levels. This information is relevant because subjects might develop different spatial biases for different difficulties (e.g. select more often the image on the right side in harder trials), making it impossible to compare different difficulty levels. The two-way ANOVA for repeated measures revealed that Type 1 criterion did not differ from zero $\left(\mathrm{F}_{1,16}=0.009, \mathrm{p}=0.93\right)$ or among the difficulty levels $\left(\mathrm{F}_{4,64}=0.248, \mathrm{p}=0.91\right)$, suggesting that subjects identified the match on the right or left side of the screen with the same probability at all difficulty levels.

Next, we performed two independent one-way ANOVAs for repeated measures to test for differences in certainty readouts across the difficulty levels. The first ANOVA 
showed that readouts of certainty of being correct decreased with difficulty level $\left(\mathrm{F}_{4,64}=11.715, \mathrm{p}<0.0001\right)$. The pair-wise post-hoc test revealed that these readouts decreased significantly only at the highest difficulty level 5 ( $p<0.05$; Fig. 11). The second ANOVA showed that readouts of certainty of being incorrect also decreased with increased trial difficulty $\left(\mathrm{F}_{4,64}=4.529, \mathrm{p}<0.01\right)$. The post-hoc test revealed that these readouts decreased significantly at the difficulty levels 4 and 5 ( $p<0.05$, Fig. 11). These results suggest that subjects were able to better read out both certainty directions when trials were easier.

\section{PDW slopes minus PreDW slopes}
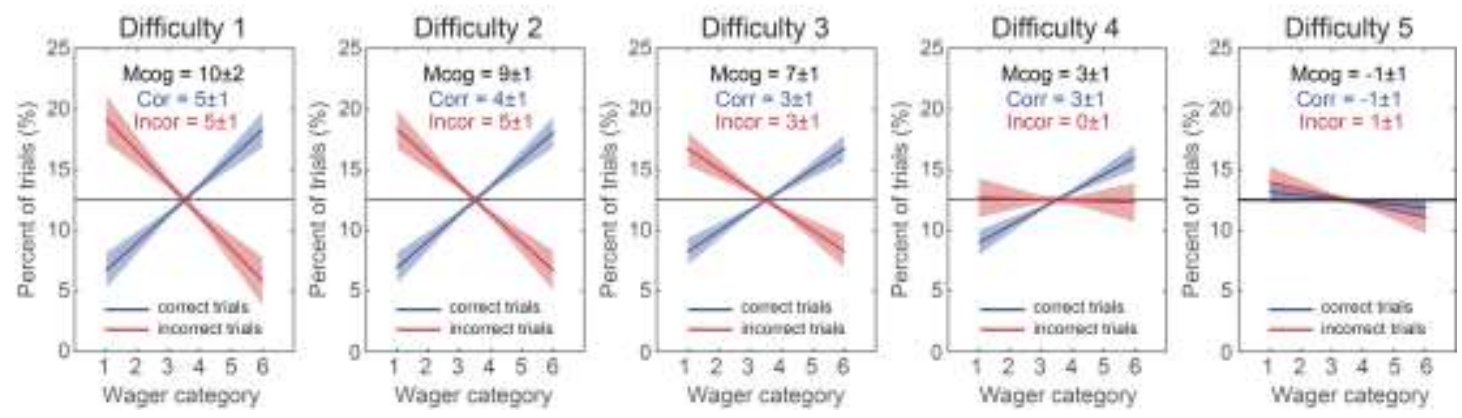

Figure 11. Means and standard errors of PDW slope-correct minus PreDW slope-correct (blue lines and shaded bands) and of PDW slope-incorrect minus PreDW slope-incorrect (red lines and shaded bands) for each difficulty level. The text in the top of each panel show means and standard errors of slope-based metacognitive ability ("Mcog"), readout of certainty of being correct ("Corr") and readout of certainty of being incorrect ("Incor").

\subsection{Earnings on PDW trials}

Finally, we applied two linear regressions to understand how subjects' PDW earnings were determined by their metacognitive abilities. The first linear regression showed that subjects' earnings can be explained by their general metacognitive ability quantified by slope-based measurements $\left(\beta=0.817, \mathrm{t}_{15}=3.686, \mathrm{p}<0.005\right)$. A second linear regression dissociated the two components of the slope-based metacognitive ability and showed that, although the ability to read out certainty of being correct partially explained their earnings $\left(\beta=0.622, \mathrm{t}_{14}=2.646, \mathrm{p}<0.05\right)$, their readouts of certainty of being incorrect influenced more how much they earned during PDW $\left(\beta=1.060, \mathrm{t}_{14}=4.201, \mathrm{p}<0.001\right)$. 


\section{Discussion}

In the present study we were able to measure trial-specific readouts of certainty of being correct and certainty of being incorrect by asking subjects to bet money on their perceptual decisions after a delayed match-to-sample task (post-decision wagering, PDW). We quantified both certainty directions with a help of pre-decision wagering (PreDW, a task in which subjects bet according to the average expected difficulty of the upcoming perceptual decision and their internal biases), isolating PDW trialspecific information. Within the same difficulty level, adaptive PDW arose from efficient readouts of varying accumulated evidence and varying Type 1 performances in different trials. Our results provide a new perspective on the interpretation of certainty scales, since they demonstrate that subjects can use these scales to report bidirectional certainty readouts if given an opportunity for such reports, even though they were not explicitly instructed to do so (see Supplementary Discussion for a detailed discussion about the influence of the task design on certainty scales).

We also established a relationship between certainty readouts and metacognitive efficiency (meta-d'/d'). Subjects with high metacognitive efficiency (meta-d'>d') were those who read out not only certainty of being correct, but also certainty of being incorrect. In addition, most subjects with high metacognitive efficiency had Type 1 performance below the chance level for the lowest wager, revealing their ability to identify incorrect Type 1 decisions. Maniscalco and Lau (2012) initially assumed that the Type 2 sensitivity (meta-d') should not exceed the Type 1 sensitivity (d') because subjects use the same evidence in both types of decision. We argue that, since trial-specific readouts of certainty of being incorrect intrinsically depend on post-decisional evidence accumulation (due to a reversal of the accumulation direction), the relationship between certainty of being incorrect and metacognitive efficiency suggests the use of extra evidence after Type 1 decisions. Hence, metacognitive efficiency can be used not only as measure of deleterious evidence leakage when meta-d' $<$ ', but also as a measure of evidence accumulation, especially when meta-d'>d'.

Altogether, our results suggest - supporting Yu et al. (2015) and Murphy et al. (2015) findings - that post-decisional evidence accumulation must be considered when Type 2 decisions follow Type 1 decisions in time. More importantly, our slope-based 
results provided the first demonstration that the interpretation of implicit certainty scales should take into account certainty bi-directionality.

The slope-based results were further supported by the reaction times during Type 2 decisions since they also suggested increased certainty in both directions of the wager scale. In previous studies that used multiple-grade scales, Type 2 reaction times were not directly associated to certainty because the starting position of motor responses used for confidence reports varied across trials to reduce motor planning. Consequently, reaction times might have also varied because the starting position could be closer or farther from the intended option, causing varying motor urgency not related to certainty (e.g. Fleming et al., 2012b; Lebreton et al., 2015). In our task design, however, Type 2 reaction times were based on binary decisions (wager high or low) and associated with specific wagers in a second response step. This novel design with two-step Type 2 decision reports allowed us to measure unbiased Type 2 reaction times in the first stage, and to provide additional support for the bidirectional interpretation of the certainty scale mapped onto the wagering scale.

\subsection{Metacognitive efficiency as a measure of evidence accumulation}

$\mathrm{Yu}$ et al. (2015) found that post-decisional evidence accumulation has asymmetric rates depending on the drift direction. According to their results, the accumulation is stronger towards the non-selected option (inconsistent evidence). We, therefore, interpret the similar asymmetry found in our group comparison - stronger association of increased metacognitive efficiency with the readout of certainty of being incorrect than with readout of certainty of being correct - as an additional demonstration that metacognitive efficiency can be linked to post-decisional evidence accumulation.

\subsection{Trial difficulty and certainty readouts}

Commonly, previous studies interpreted low confidence reports as low certainty (Fleming and Lau, 2014; Heereman et al., 2015; Maniscalco and Lau, 2012; Sandberg et al., 2010). In those cases, low certainty would be expected to increase on harder trials together with the use of low ratings. However in the present study, certainty of being incorrect increased on easier trials (Fig. 11) indicating that, on these trials, 
subjects were using low wagers when they were more certain about their (incorrect) decisions, and not when they were more uncertain.

\subsection{Revisiting our framework}

We bring together our results about certainty bi-directionality, post-decisional evidence accumulation and trial difficulty, and the knowledge provided by published models of evidence accumulation (e.g. Pleskac and Busemeyer, 2010; Yeung and Summerfield, 2012; Yu et al., 2015), to update the framework presented in scenario C of the Figure 1.

Figure 12 illustrates one harder (A) and two easier (B) incorrect trials of the DMTS task followed by six-grade certainty scale exemplified in the Figure 1 (see Introduction for more details about the trial dynamics). The "evidence axis" exemplifies a theoretical range of evidence available for the task. On harder trials, the evidence is distributed narrowly around the Type 1 criterion. The easier the trial, the higher the probability that the evidence is accumulated further from the Type 1 criterion, and closer to the limits of the evidence axis. In a specific trial, if the postdecisional evidence remains in the same side of the Type 1 criterion as during the Type 1 decision, it is read out as certainty of being correct (blue curve). If the evidence crosses the Type 1 criterion, it leads to different levels of certainty of being incorrect (red curve). Hence, certainty increases towards the two directions of the evidence axis, resulting in a bi-directional certainty scale as the exemplified U-shape function of certainty at the bottom of the figure.

On harder trials (Fig. 12A), the difference between the perceptual evidence supporting each option is small, and consequently d'. According to our results, the Type 1 performance at the hardest difficulty level was at the chance level. Moreover, subjects did not show any metacognitive ability during Type 2 decisions in most difficult trials (Fig. 11). We suggest, therefore, that the post-decisional evidence accumulation rate remained as low as before the Type 1 decisions, and did not allow subjects to improve their metacognitive efficiency. On easier trials (Fig. 12B), the large difference between the perceptual evidence supporting each option of the Type 1 decision allowed subjects to better distinguish the match, generating a high d'. Nevertheless, there were still some trials, as exemplified, in which subjects selected the wrong 
option. Our group comparisons suggest that subjects with low metacognitive efficiency did not accumulate post-decisional evidence or failed in reading it out efficiently, since their meta-d' was smaller than d' (Fig. 12B, left). We suggest that subjects with high metacognitive efficiency accumulated more evidence after the Type 1 decisions (Fig. 12B, right). The post-decisional evidence accumulation allowed subjects with high metacognitive efficiency to improve the detection of correct choices (not shown in this figure), but impacted even more the detection of the incorrect choices.

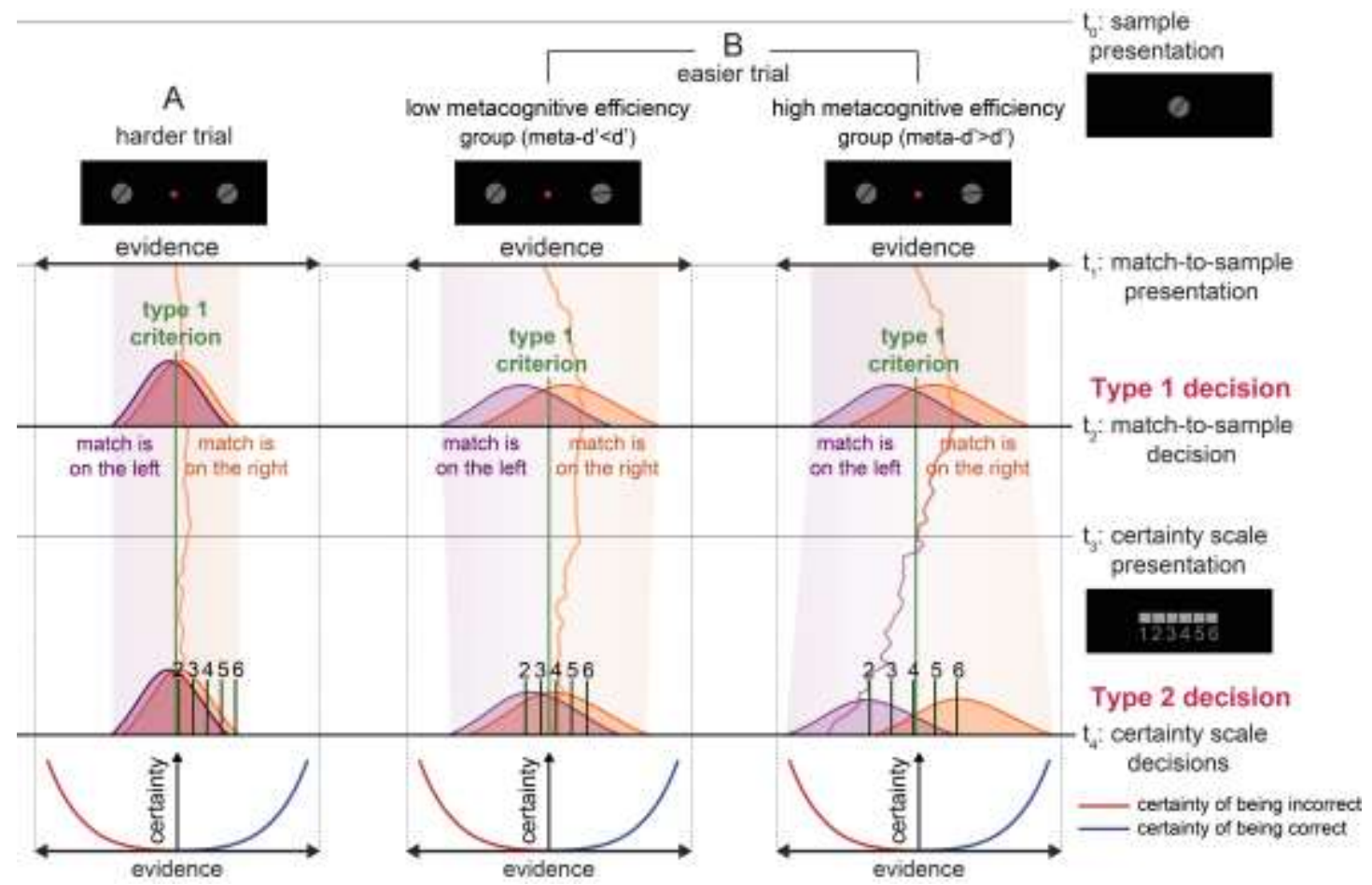

Figure 12. Framework of scenario C (Fig. 1C) revised according to our findings and to published models of evidence accumulation. Trial time goes down from $t_{0}$ to $t_{4}$. (A) On harder trials, $d$ ' is small and the drift rate of evidence accumulation before and after the Type 1 decision is low, resulting in Type 1 performance at the chance level and no metacognitive ability. The Type 2 criteria distribution is biased to the use of low ratings, which in this case signify the low certainty. (B) On easier trials, the accumulation of evidence before the Type 1 decision is high and, consequently, $\mathrm{d}^{\prime}$ is large. We propose that the inter-subject variability in metacognitive efficiency arises from their ability to accumulate and read out post-decisional evidence. On the left, although subjects with low metacognitive efficiency have the same d' as subjects with high metacognitive efficiency, they did not accumulate postdecisional evidence fast enough or failed in reading it out efficiently since their meta-d' is smaller than d'. On the right, the post-decisional evidence accumulation is high for subjects with high metacognitive efficiency, allowing them to better distinguish correct from incorrect Type 1 decisions and increase 
their meta-d'. Compared to the low metacognitive efficiency group, the post-decisional accumulation of evidence is especially high when it drifts toward the non-selected match option, more strongly influencing the detection of the incorrect choices. Importantly, in this case, low ratings signify a high certainty (of being incorrect), rather than low certainty.

It is important to emphasize that the level of certainty each rating (or wager) represents critically depends on the difficulty level and on individual ability to accumulate and read out evidence. On harder trials, subjects more often used low wagers and, due to low average certainty (i.e. high difficulty level), these wagers represented low levels of trial certainty. On easier trials, subjects used more often high wagers because of high average certainty (i.e. low difficulty level) but, in this case, low wagers represented high or low levels of trial certainty depending on subjects' metacognitive efficiency. Subjects with high metacognitive efficiency were able to accumulate inconsistent post-decisional evidence at high rates. This evidence not only could cross the Type 1 criterion, but also reach far distances to the other side. The readouts of this evidence generated high certainty of being incorrect, which was mainly reported through the lowest wager. Subjects who did not accumulate inconsistent post-decisional evidence at high rates, on the other hand, used low wagers to report certainty readouts about the evidence that was close to the Type 1 criterion (i.e. low certainty level). This reasoning further emphasizes that the same response scale can be used to represent very different metacognitive readouts, underscoring the importance of interpretation in addition to scale formulation. Furthermore, the dependence of certainty representations on the difficulty and individual biases endorses the importance of the baseline measures to distinguish between trial-specific and average certainty readouts.

\section{Summary}

The present work contributes to the idea that humans and other animals continue to monitor and accumulate relevant evidence even after the decision, to plan future actions more efficiently. Most importantly, it suggests that continued accumulation of evidence allows subjects to read out and report two certainty directions: certainty of being correct and certainty of being incorrect, provided that the metacognitive response scale affords the possibility of such reports. 
One of the strengths of our task design and analyses is the possibility of studying these bi-directional certainty readouts not only in humans but also in other animals, as well as in patients with moderate impairments on language comprehension. We inherited this strength from the reward-driven post-decision wagering approach, and we expanded its advantages by using concomitantly pre- and post-decision wagering to isolate trial-specific information and access certainty of being correct and certainty of being incorrect separately. 


\section{Supplementary material}

\section{S.1 Supplementary Results}

The Supplementary Figure 1A and Supplementary Figure 1B illustrate - for PreDW and PDW, respectively - the wager-specific proportions of correct and incorrect trials separately for the five difficulty levels. These proportions resulted in the slope-based measures illustrated in the third row (Supplementary Figure 1C) of this figure and in the Figure 11. To test, in each difficulty level, if slope-correct was different from slope-incorrect depending on the trial type (PreDW or PDW), we performed two-way ANOVAs for repeated measures. In none of the difficulty levels PreDW slope-correct was significantly different from PreDW slope-incorrect ( $p>0.05$; Supplementary Fig. 1A), indicating reliable baselines.

Next, we performed paired t-tests within each difficulty level to test for significant differences between PDW and PreDW slopes. The t-tests revealed that subjects' PDW slopes-correct were different from their PreDW slopes-correct at all difficulty levels $(\mathrm{p}<0.01)$, except for the highest difficulty level 5 ( $\mathrm{p}>0.05$ ). Additionally, PDW slopeincorrect was not different from PreDW slope-incorrect for the difficulty levels 4 and 5 ( $>0.05$ ), while subjects were able to read out certainty of being incorrect at the difficulty levels 1,2 and $3(\mathrm{p}<0.01)$.

The Supplementary Figure 1 revealed two important points considered in our analyses. First: the baseline condition (PreDW trials) revealed the general (non-trialspecific) effect of expected perceptual difficulty assessments. For instance, the realization of increased family difficulty made subjects to use low wagers increasingly but independently of the correctness of the trials. Without considering this baseline measurement, we would not be able to distinguish this adaptive strategy (wagering low for hard trials) from the use of trial-specific certainty during PDW trials. Even if the task would contain only one difficulty level, or maybe especially in those cases, the baseline measurement is essential to quantify certainty readouts taking into account individual biases. Second: during PreDW trials (especially during easier trials) subjects chose more often middle wagers (wagers 3 and 4). Since linear fits captured wagering trends regardless of the effect of such behavior, our choice for using the slopes of linear fits (instead of the best-fitted curves) proved to be valuable for establishing useful baselines for the slope-based measurements. 
A
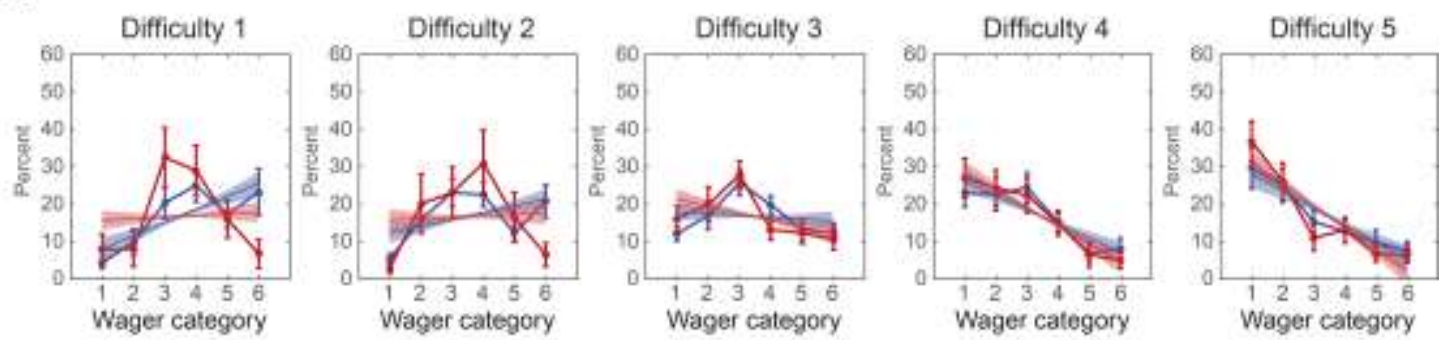

B

Post-decision wagering (PDW)
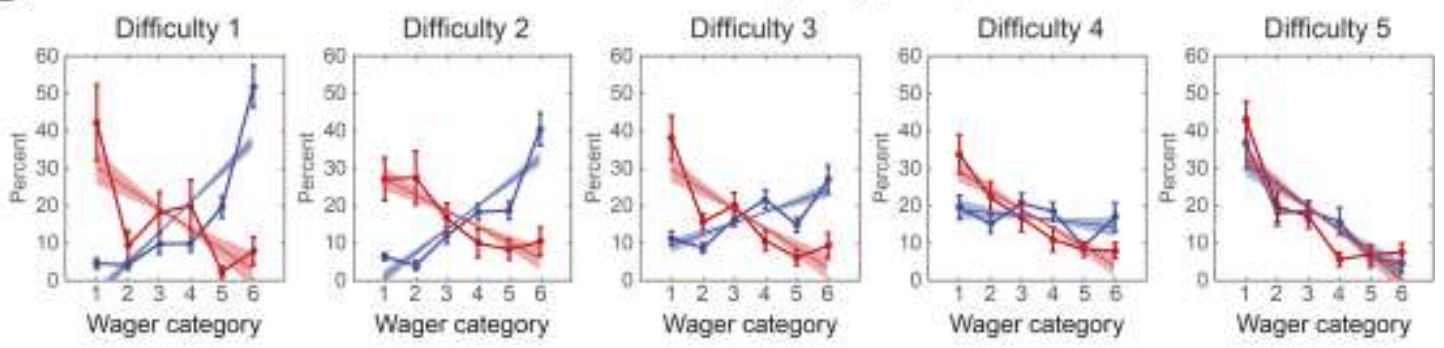

C
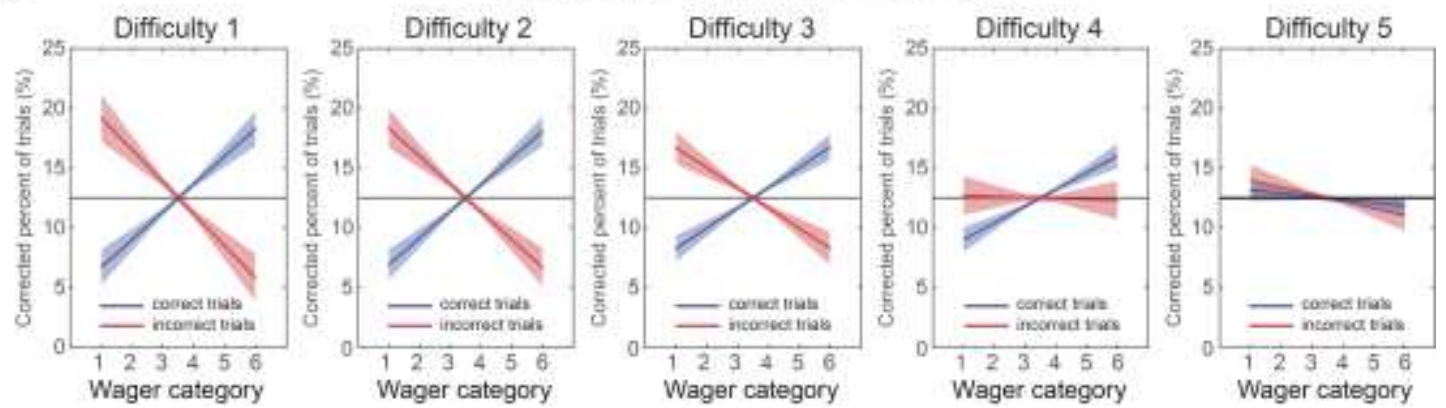

Supplementary Figure 1. (A) Means and standard errors of linear fits for correct trials (blue lines and shaded bands) and incorrect trials (red lines and shaded bands) for (A) PreDW (baseline) and (B) PDW, fitted to the data: means and standard errors of wager-specific proportion of correct (blue curves) and incorrect (red curves) trials, for each difficulty level. (C) Means and standard errors of PDW slope-correct minus PreDW slope-correct (blue line and band) and of PDW slope-incorrect minus PreDW slope-incorrect (red line and band) for each difficulty level (same data as in Figure 11). Measurements represent averages across subjects.

The frequency of the use of each wager (means and standard errors from the lowest to the highest wager) in PDW trials (19 $\pm 211 \pm 1 \quad 16 \pm 1 \quad 16 \pm 213 \pm 125 \pm 3 \%)$ and in PreDW

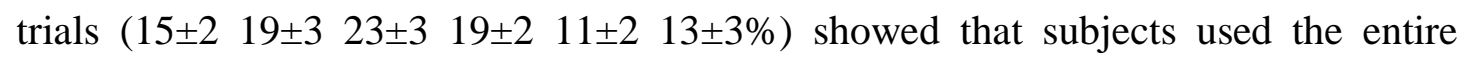
wager scale. 


\section{S.2 Supplementary Discussion}

Different aspects of the task design might favor or limit post-decisional evidence accumulation and, consequently, bi-directional certainty readouts. We discuss these aspects below together with our results.

Time pressure: Yeung and Summerfield (2012) proposed that later drifts towards the correct option, not considered by the time subjects commit with the wrong option, generate extra evidence used for error detection. In Charles et al. (2013), for example, subjects who needed to report Type 1 decisions within $1 \mathrm{~s}$ showed higher metacognitive efficiency than those who had twice the time to report their perceptual decisions. This result suggests, according to the link we propose between metacognitive efficiency and post-decisional evidence accumulation, that time pressure over Type 1 decisions increased the use of extra information during Type 2 decisions. In our experiment, the average readout of certainty of being incorrect and the predominance of subjects with meta-d'>d' indicate that the applied time pressure (1.5 s) was enough to restrict evidence accumulation before Type 1 decisions and favor post-decisional evidence accumulation.

Memory: memory tasks (such as DMTS) might promote the use of short-term memory as another source of extra information for the Type 2 decisions. Since the basic information required for these Type 1 decisions, the sample, can only be accessed through top-down memory retrieval mechanisms, those mechanisms - which do not depend exclusively on continuous evidence input - might keep providing information also after Type 1 decisions (Magnussen and Greenlee, 1999; Yu et al., 2015).

Propriosensory evidence: the propriosensory evidence related to the report of the Type 1 decision itself can serve as another source of post-decisional information. It is known that Type 1 reaction times correlate with certainty (Fetsch et al., 2014; Kiani and Shadlen, 2009). Therefore, subjects could, in principle, read out those reaction times - instead of or in addition to the perceptual evidence - in order to judge their

Type 1 performance during the Type 2 decisions. In accordance to this reasoning, Fleming (2015) modified subjects' Type 2 decisions by manipulating the activity of motor areas prior to metacognitive reports. In our experiment, on the contrary, wagerspecific Type 1 reaction times were unidirectional (decreased towards the highest 
wager) and could not provide the bi-directional readouts measured both by the slopebased measurements and by the wager-specific Type 2 reaction times.

Rewards and punishments: lastly, the use of PDW might favor evidence accumulation because it motivates subjects to fully explore their sources of information through rewards and punishments (i.e. profit more when correct and avoid losses when incorrect). We suggest that the monetary motivational aspect of PDW also contributed to the predominance of subjects showing high metacognitive efficiency (11 out of 17 subjects).

Taking into account the potential influence of the described aspects on the postdecisional evidence accumulation, it still needs to be tested how the bi-directionality of the certainty scale is affected by different task designs. Nevertheless, we presented data to argue that post-decisional evidence accumulation should be considered in experiments in which certainty readouts are required after speeded Type 1 decisions. 


\section{References}

Boldt, A., \& Yeung, N. (2015). Shared Neural Markers of Decision Confidence and Error Detection. Journal of Neuroscience, 35(8), 3478-3484. http://doi.org/10.1523/JNEUROSCI.0797-14.2015

Clarke, F. R., Birdsall, T. G., \& Tanner Jr, W. P. (1959). Two types of ROC curves and definitions of parameters. The Journal of the Acoustical Society of America, 31(5), 629-630.

Charles, L., Van Opstal, F., Marti, S., \& Dehaene, S. (2013). Distinct brain mechanisms for conscious versus subliminal error detection. NeuroImage, 73, 80-94. http://doi.org/10.1016/j.neuroimage.2013.01.054

Fetsch, C. R., Kiani, R., Newsome, W. T., \& Shadlen, M. N. (2014). Effects of Cortical Microstimulation on Confidence in a Perceptual Decision. Neuron, 84(1), 239. http://doi.org/10.1016/j.neuron.2014.09.020

Fleming, S. M., Dolan, R. J., \& Frith, C. D. (2012a). Metacognition: computation, biology and function. Philosophical Transactions of the Royal Society B: Biological Sciences, $\quad 367(1594), \quad 1280-1286$. http://doi.org/10.1098/rstb.2012.0021

Fleming, S. M., Huijgen, J., \& Dolan, R. J. (2012b). Prefrontal Contributions to Metacognition in Perceptual Decision Making. Journal of Neuroscience, 32(18), 6117-6125. http://doi.org/10.1523/JNEUROSCI.6489-11.2012

Fleming, S. M., \& Lau, H. C. (2014). How to measure metacognition. Frontiers in Human Neuroscience, 8. http://doi.org/10.3389/fnhum.2014.00443

Fleming, S. M., Maniscalco, B., Ko, Y., Amendi, N., Ro, T., \& Lau, H. (2015). Action-specific disruption of perceptual confidence. Psychological Science, 26(1), 89-98.

Galvin, S. J., Podd, J. V., Drga, V., \& Whitmore, J. (2003). Type 2 tasks in the theory of signal detectability: Discrimination between correct and incorrect decisions. Psychonomic Bulletin \& Review, 10(4), 843-876.

Gold, J. I., \& Shadlen, M. N. (2001). Neural computations that underlie decisions about sensory stimuli. Trends in Cognitive Sciences, 5(1), 10-16. 
Hampton, R. R. (2001). Rhesus monkeys know when they remember. Proceedings of the National Academy of Sciences, 98(9), 5359-5362.

Hampton, R. R. (2009). Multiple demonstrations of metacognition in nonhumans: Converging evidence or multiple mechanisms? Comparative Cognition \& Behavior Reviews, 4, 17.

Hebart, M. N., Schriever, Y., Donner, T. H., \& Haynes, J.-D. (2014). The Relationship between Perceptual Decision Variables and Confidence in the Human Brain. Cerebral Cortex, 26(1), 118-130. http://doi.org/10.1093/cercor/bhu181

Heereman, J., Walter, H., \& Heekeren, H. R. (2015). A task-independent neural representation of subjective certainty in visual perception. Frontiers in Human Neuroscience, 9. http://doi.org/10.3389/fnhum.2015.00551

Kepecs, A., Uchida, N., Zariwala, H. A., \& Mainen, Z. F. (2008). Neural correlates, computation and behavioural impact of decision confidence. Nature, 455(7210), 227-231. http://doi.org/10.1038/nature07200

Kepecs, A. (2013). The uncertainty of it all. Nature Neuroscience, 16(6), 660-662.

Kepecs, A., \& Mainen, Z. F. (2012). A computational framework for the study of confidence in humans and animals. Philosophical Transactions of the Royal Society B: Biological Sciences, 367(1594), 1322-1337. http://doi.org/10.1098/rstb.2012.0037

Kiani, R., Corthell, L., \& Shadlen, M. N. (2014). Choice Certainty Is Informed by Both Evidence and Decision Time. Neuron, 84(6), 1329-1342. http://doi.org/10.1016/j.neuron.2014.12.015

Kiani, R., \& Shadlen, M. N. (2009). Representation of Confidence Associated with a Decision by Neurons in the Parietal Cortex. Science, 324(5928), 759-764. http://doi.org/10.1126/science.1169405

Lebreton, M., Abitbol, R., Daunizeau, J., \& Pessiglione, M. (2015). Automatic integration of confidence in the brain valuation signal. Nature Neuroscience, 18(8), 1159-1167. http://doi.org/10.1038/nn.4064

Magnussen, S., \& Greenlee, M. W. (1999). The psychophysics of perceptual memory. Psychological Research, 62(2-3), 81-92. 
Maniscalco, B., \& Lau, H. (2012). A signal detection theoretic approach for estimating metacognitive sensitivity from confidence ratings. Consciousness and Cognition, 21(1), 422-430. http://doi.org/10.1016/j.concog.2011.09.021

Ma, W. J., \& Jazayeri, M. (2014). Neural Coding of Uncertainty and Probability. Annual Review of Neuroscience, 37(1), 205-220. http://doi.org/10.1146/annurev-neuro-071013-014017

Monosov, I. E., \& Hikosaka, O. (2013). Selective and graded coding of reward uncertainty by neurons in the primate anterodorsal septal region. Nature Neuroscience, 16(6), 756-762. http://doi.org/10.1038/nn.3398

Moran, R., Teodorescu, A. R., \& Usher, M. (2015). Post choice information integration as a causal determinant of confidence: Novel data and a computational account. Cognitive Psychology, 78, 99-147. http://doi.org/10.1016/j.cogpsych.2015.01.002

Murphy, P. R., Robertson, I. H., Harty, S., \& O’Connell, R. G. (2015). Neural evidence accumulation persists after choice to inform metacognitive judgments. eLife, e11946. http://doi.org/10.7554/eLife.11946

Persaud, N., McLeod, P., \& Cowey, A. (2007). Post-decision wagering objectively measures awareness. Nature Neuroscience, 10(2), 257-261. http://doi.org/10.1038/nn1840

Pinheiro, J., Bates, D., DebRoy, S., \& Sarkar, D. (2007). nlme: Linear and Nonlinear Mixed Effects Models. R package version 3.1-115; 2014.

Pleskac, T. J., \& Busemeyer, J. R. (2010). Two-stage dynamic signal detection: A theory of choice, decision time, and confidence. Psychological Review, 117(3), 864-901. http://doi.org/10.1037/a0019737

Pouget, A., Drugowitsch, J., \& Kepecs, A. (2016). Confidence and certainty: distinct probabilistic quantities for different goals. Nature Neuroscience, 19(3), 366374. http://doi.org/10.1038/nn.4240

Sandberg, K., Timmermans, B., Overgaard, M., \& Cleeremans, A. (2010). Measuring consciousness: Is one measure better than the other? Consciousness and Cognition, 19(4), 1069-1078. http://doi.org/10.1016/j.concog.2009.12.013 
Van den Berg, R., Anandalingam, K., Zylberberg, A., Kiani, R., Shadlen, M. N., \& Wolpert, D. M. (2016). A common mechanism underlies changes of mind about decisions and confidence. Elife, 5, e12192.

Yeung, N., \& Summerfield, C. (2012). Metacognition in human decision-making: confidence and error monitoring. Philosophical Transactions of the Royal Society B: Biological Sciences, 367(1594), 1310-1321. http://doi.org/10.1098/rstb.2011.0416

Yu, S., Pleskac, T. J., \& Zeigenfuse, M. D. (2015). Dynamics of postdecisional processing of confidence. Journal of Experimental Psychology: General, 144(2), 489-510. http://doi.org/10.1037/xge0000062 


\section{Chapter 2}

Multiple brain areas encode certainty in a bi-directional way 


\section{Table of contents}

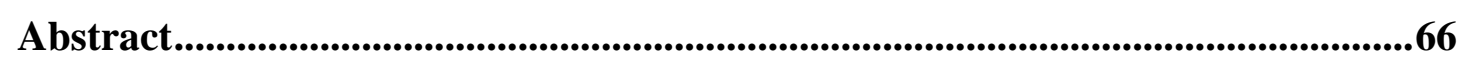

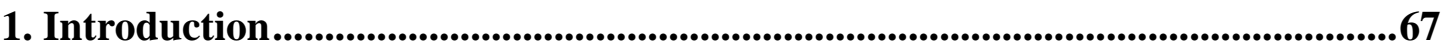

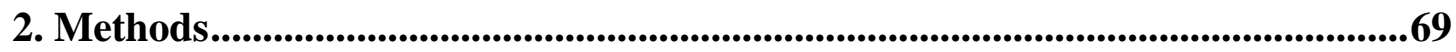

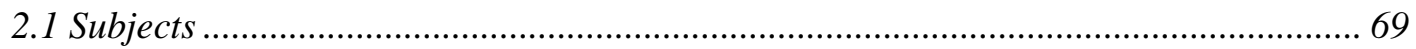

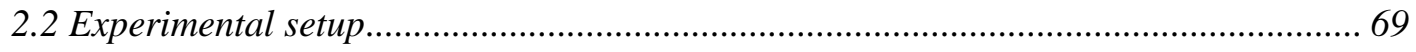

2.3 Perceptual decisions: delayed match-to-sample task ................................................ 70

2.4 Confidence scale: post-decision wagering ................................................................ 70

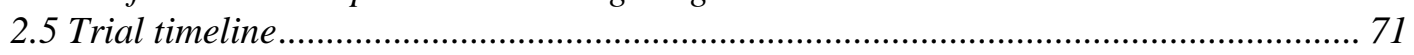

2.6 Time-course of experiment: practice and experimental sessions............................... 73

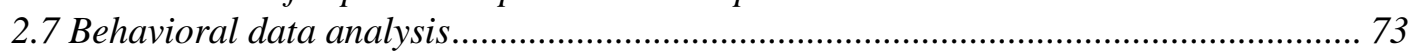

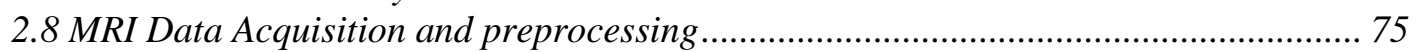

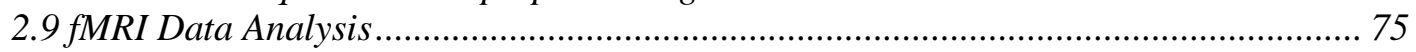

2.9.1 Exponential relationship between wagering and brain activation ........................... 78

2.9.2 Additional post-hoc tests for regions of interest ........................................................ 79

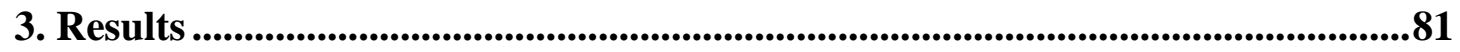

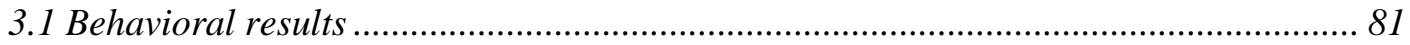

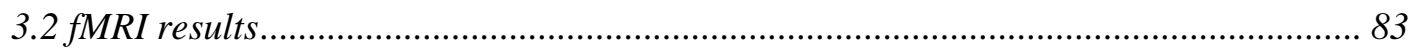

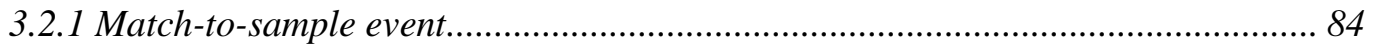

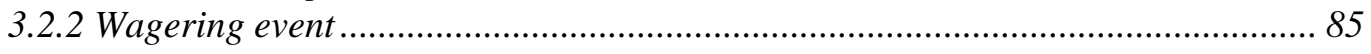

3.2.3 Overlap between areas encoding difficulty levels and certainty .......................... 89

3.2.4 The post-match-to-sample event ........................................................................ 90

3.3 Correlations between behavioral measures and brain activity................................... 91

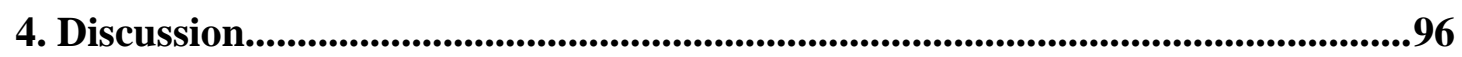

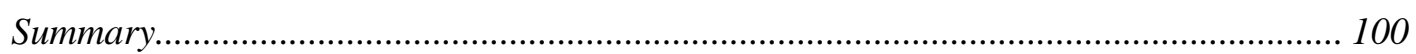

Supplementary information ...........................................................................................102

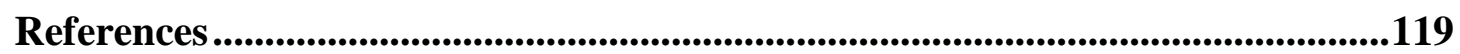




\begin{abstract}
Humans and other animals continuously evaluate their decisions in order to behave adaptively. If the post-decisional information is inconsistent with the information used during the decision, it might decrease subject's confidence about having made a correct decision (certainty of being correct), or even make them confident about having made an incorrect decision (certainty of being incorrect). Using six-grade post-decision wagering, we previously demonstrated that many subjects are able to read out both certainty directions to flexibly bet money on the accuracy of their perceptual decisions. The present study aimed at locating the brain areas that encode these readouts by asking subjects to perform a similar task inside an MRI scanner. We show that the behavioral requirements of the post-decision wagering task (gain more when correct and avoid large losses when incorrect) prompted several brain areas such as the dorsolateral prefrontal cortex, the dorsal anterior cingulate cortex and the intraparietal sulcus - to encode certainty in a bi-directional way during the wagering response period. Fewer areas encoded exclusively either unidirectional certainty of being correct or certainty of being incorrect. Moreover, we demonstrated that the areas involved in the bi-directional certainty readouts did not encode the difficulty of the perceptual decisions. Nevertheless, some of these areas were involved in the evaluation of post-decisional information prior to the wagering event, which might have resulted in the certainty-related bi-directional reports. Our results demonstrate that under behavioral demands that prompt, and afford, an adaptive strategy of implicit metacognitive judgments utilizing both certainty directions, the encoding of the bi-directional certainty becomes a prevalent pattern distributed across task-related brain regions.
\end{abstract}

Keywords: bi-directional certainty, confidence, post-decisional information, reward expectation, fMRI, post-decision wagering

Abbreviations: post-decision wagering (PDW), delayed match-to-sample task (DMST) 


\section{Introduction}

Adaptive interaction with the environment requires that humans and other animals evaluate their decisions continuously. This is possible because individuals accumulate evidence during decision-making processes (Ratcliff and McKoon, 2008) as well as after decisions are made, even if decision outcomes are not accessed promptly (Fleming, 2016; Murphy et al., 2015; Pleskac and Busemeyer, 2010; Yu et al., 2015). The amount of evidence supporting one decision option is commonly referred to as decision variable (DV) and is reflected in the certainty that individuals have about their decisions. In this context, binary perceptual decisions are thought to be implemented by comparing DV against the criterion that subjects use to classify the evidence (i.e. decision criterion), whereas the certainty would reflect the readout of the distance between DV and this criterion (De Martino et al., 2012; Hebart et al., 2014; Kiani and Shadlen, 2009; Moran et al., 2015). Consequently, certainty would vary as individuals accumulate more evidence (Pleskac and Busemeyer, 2010; Yu et al., 2015).

At the post-decisional stage, accumulated evidence can represent error likelihood if it is conflicting with the information used during the decision (Moran et al., 2015; Murphy et al., 2015; Taylor et al., 2015). Hence, post-decisional information not only modifies the decision reliability (i.e. its certainty level), but it can also be classified as consistent or inconsistent ( $\mathrm{Yu}$ et al., 2015). The consistency of the post-decisional evidence refers to its drift direction. When the post-decisional information drifts to the same direction of the pre-decisional drift (consistent information), it increases certainty of being correct. When it drifts to the opposite direction of the predecisional drift (inconsistent information), it decreases certainty of being correct. We named as certainty of being incorrect the inconsistent information that crosses the decision criterion and thus starts supporting the non-selected decision option (Moreira et al., 2016).

Although certainty has recently become one of the most hotly debated topics in the neuroscience of decision-making (Fleming, 2016; Pouget et al., 2016), the focus of certainty-related studies has been so far on certainty of being correct (Fleming and Lau, 2014; Fleming et al., 2012; Hebart et al., 2014; Heereman et al., 2015; Kepecs and Mainen, 2012; Kiani and Shadlen, 2009). Most of these studies consider that certainty readouts are essentially unidirectional, neglecting the possibility that DV 
crosses the decision criterion after the decisions. However, in a previous study we have demonstrated that human subjects are able to wager adaptively after their decisions (post-decision wagering, PDW; Persaud et al., 2007) by reading out certainty of being correct and certainty of being incorrect in order to profit more when the decision was correct and avoid large losses when it was incorrect (Moreira et al., 2016). Since we have shown that certainty readouts can be bi-directional, the aim of the present study was to identify brain areas that encode both readouts. To this end, we used a task with similar perceptual decisions (match-to-sample) and implicit certainty scale (six-grade PDW) components as in Moreira et al. (2016), adapted for slow event-related fMRI. Three parametric models were created to search for brain areas able to encode (a) bi-directional certainty, (b) unidirectional certainty of being correct or (c) certainty of being incorrect.

One hypothesis is that different brain areas are associated with unidirectional readouts of certainty of being correct and certainty of being incorrect, and the information associated with each certainty direction is only converged at the late stages of brain processing (e.g. movement coding at the primary motor cortex). In previous studies, the readouts of certainty of being incorrect were more robustly associated with areas of the cingulate cortex (e.g. dorsal anterior cingulate cortex in Charles et al., 2013 and Taylor et al., 2015; posterior cingulate cortex in Agam et al., 2011), whereas the association between readouts of certainty of being correct and brain activity was investigated by a greater number of studies, and assigned to innumerous areas (e.g. anterior prefrontal cortex in Fleming et al., 2012; ventral striatum, dorsal anterior cingulate cortex and superior parietal lobule in Hebart et al., 2014; dorsolateral prefrontal cortex in Heereman et al., 2015; pulvinar in Komura et al., 2013; ventromedial prefrontal cortex in De Martino et al., 2012; intraparietal sulcus in Filimon et al., 2013; premotor cortex in Fleming et al., 2015). Hence, if the brain processes each certainty direction mostly independently, those areas would be separately associated with each certainty direction.

As an alternative hypothesis, cortical and subcortical areas could encode both certainty directions. This hypothesis is in agreement with the distributed consensus model of Cisek and Kalaska (2010) which proposes that the brain implements multiple action options and decides for the most adaptive one using many levels of representations already within the sensorimotor system. This model fits to our 
experiment because PDW is not only influenced by evidence-related certainty readouts, but it is also associated with subjective cost functions typical of rewardbased decisions that have ecological consequences to the subjects (De Martino et al., 2012; Kepecs and Mainem, 2012; Pouget et al., 2016). We hypothesized that, in our experiment, if certainty-related information influences representations of action options, a distributed processing would be reflected in a bi-directional pattern of activity in multiple brain areas during the wagering period.

In accordance with the distributed hypothesis, we found out that several frontal, parietal, cingulate and subcortical areas presented bi-directional pattern of activity during PDW. Therefore, the present work is the first demonstration of a distributed processing based on bi-directional certainty information, resulting from adaptive implicit metacognitive judgments driven by task demands.

\section{Methods}

\subsection{Subjects}

Twenty healthy subjects ( 5 males; mean age $25.5 \pm 3.5$ years) were recruited via the student online platform of the University of Goettingen. All subjects had normal or corrected-to-normal vision. Subjects were compensated with $10 €$ for the practice session and according to their performance during the scanning sessions (see below). All subjects provided written consent for participation. The experimental procedures were approved by the local ethics committee of the Georg-Elias-Müller-Institute for Psychology.

\subsection{Experimental setup}

Subjects reclined inside the MRI scanner in the supine position. The stimuli were back-projected on a translucent screen (800 x 600 resolution) and viewed by the subjects through a surface mirror mounted on the head coil at approximately $12 \mathrm{~cm}$ from subjects' eyes and $81 \mathrm{~cm}$ from the screen. Subjects maintained central fixation throughout the trial. Gaze position was acquired with $60 \mathrm{~Hz}$ MR-compatible miniature infrared eye tracker camera and ViewPoint 2.8.6.21 software (Arrington Research). Subjects responded with their right and left thumbs using the buttons 1 and 4 of a MR-compatible button box with 4 buttons (Current Designs). The task was 
controlled via MATLAB (Mathworks) using the Psychophysics toolbox (http://psychtoolbox.org/).

\subsection{Perceptual decisions: delayed match-to-sample task}

Subjects performed a delayed match-to-sample (DMTS) task in which they had to find, between two options, the match for a sample that consisted of a gray circle of $4^{\circ}$ of visual angle radius with a black bar crossing its center. Ten different sample options were generated by varying the bar orientation in counterclockwise rotation from the horizontal plane (from $16^{\circ}$ to $52^{\circ}$ ). One of these samples was presented pseudo-randomly at the beginning of each trial in the center of the screen. Following sample presentation, one sample-like image was presented $10.4^{\circ}$ to the right and another one $10.4^{\circ}$ to the left of the center of the screen. One of them had a bar in the same orientation as the sample (match) and the other one had a bar in a different orientation (non-match). Five difficulty levels were created by different orientation contrasts between the match and the non-match (from $4^{\circ}$ to $20^{\circ}$; Fig. 1C). Subjects selected the match by using the button positioned in the same side of the screen as the selected image (Fig. 1A).

\subsection{Confidence scale: post-decision wagering}

We used post-decision wagering (PDW) as confidence scale because it is more intuitive and motivating to subjects (Persaud et al., 2007) and because it implicitly prompts individuals to read out both certainty directions and report them using the wagers (Moreira et al., 2016). During PDW, subjects bet money on the correctness of their perceptual decision. They won the wagered money for correct perceptual decisions and lost it for incorrect decisions. Subjects wagered by first selecting to wager high (wager categories 4, 5 and 6) or low (wager categories 1, 2 and 3), and afterwards by selecting a specific wager among high or low options. High or low wagers were positioned at $9.2^{\circ}$ to the right and $9.2^{\circ}$ to the left from the fixation spot, on the horizontal axis. The high and low sides varied randomly across the trials.

In $20 \%$ of the trials, subjects performed a control task instead of PDW. During the control task subjects were instructed by a blue square to select a specific "wager" (Fig. 1). Control trials aimed to dissociate general processes (i.e. visual stimulation 
and motor responses) from the investigated certainty-related processes and did not influence subject earnings.

Task instructions aimed to improve the discriminatory power of metacognitive judgments across trials by emphasizing that subjects should try to utilize the entire wagering scale. Subjects started each scanning session with $10 €$ and could earn up to $20 €$ according to their performance. They wagered on the correctness of perceptual decisions using the following pay-off matrix, which was explained to them before the experiment:

\section{Table 1}

Wagering pay-off matrix

\begin{tabular}{|c|c|c|c|c|c|c|}
\hline \multirow{2}{*}{ Perceptual decision } & \multicolumn{3}{|c|}{ Low wagers } & \multicolumn{3}{c|}{ High wagers } \\
\hline Correct & 3 cents & 7 cents & 11 cents & 15 cents & 19 cents & 23 cents \\
\hline Incorrect & -3 cents & -7 cents & -11 cents & -18 cents & -22 cents & -26 cents \\
\hline
\end{tabular}

As can be seen from the pay-off matrix, if subjects wagered low, they were rewarded and punished in the same way for correct and incorrect perceptual decisions. But when they wagered high, their incorrect decisions were punished with 3 cents more than they would be rewarded for correct decisions. This pay-off matrix was designed based on Moreira et al. (2016) in order to avoid the indiscriminate use of high wagers when subjects knew they were performing generally above the chance level (50\%).

\subsection{Trial timeline}

Eye and hand movements were controlled throughout the trial. Each trial started with the appearance of a red spot $\left(0.6^{\circ}\right.$ radius) and a gray framed-square (size $\left.5 \times 5^{\circ}\right)$ in the center of the screen. Subjects were correctly positioned in the "rest position" when they fixated the gaze inside the eye fixation window ( $3^{\circ}$ radius) and, concomitantly, positioned the left and right thumbs over the buttons 1 and 4, respectively. After the rest position period $1(3 / 4 / 5 \mathrm{~s})$, the sample was presented in the center of the screen for $1 \mathrm{~s}$, followed by a mask presented at the same position for the same amount of time. After mask presentation, subjects maintained the rest position for $3 \mathrm{~s}$ (rest position period 2) before the match-to-sample presentation, in which they had up to $1.5 \mathrm{~s}$ to select the image they believed was the match by using the button (and hand) 
corresponding to the selected side (Fig 1.A). The rest position period $3(7 / 8 / 9 / 10 \mathrm{~s})$ followed the perceptual decision. After that, subjects selected one wager category within $2.7 \mathrm{~s}$. A green square frame appearing above the selected wager for $0.3 \mathrm{~s}$ signalized the end of the wagering period. The rest position period 4 (7/8/9/10 s) occurred after PDW/control and was followed by an intertrial interval of $2 \mathrm{~s}$, in which subjects were allowed to move or close the eyes. During the rest position periods, there was blink tolerance of $0.4 \mathrm{~s}$. There was no trial-by-trial feedback about the correctness of the perceptual decisions (Fig. 1).

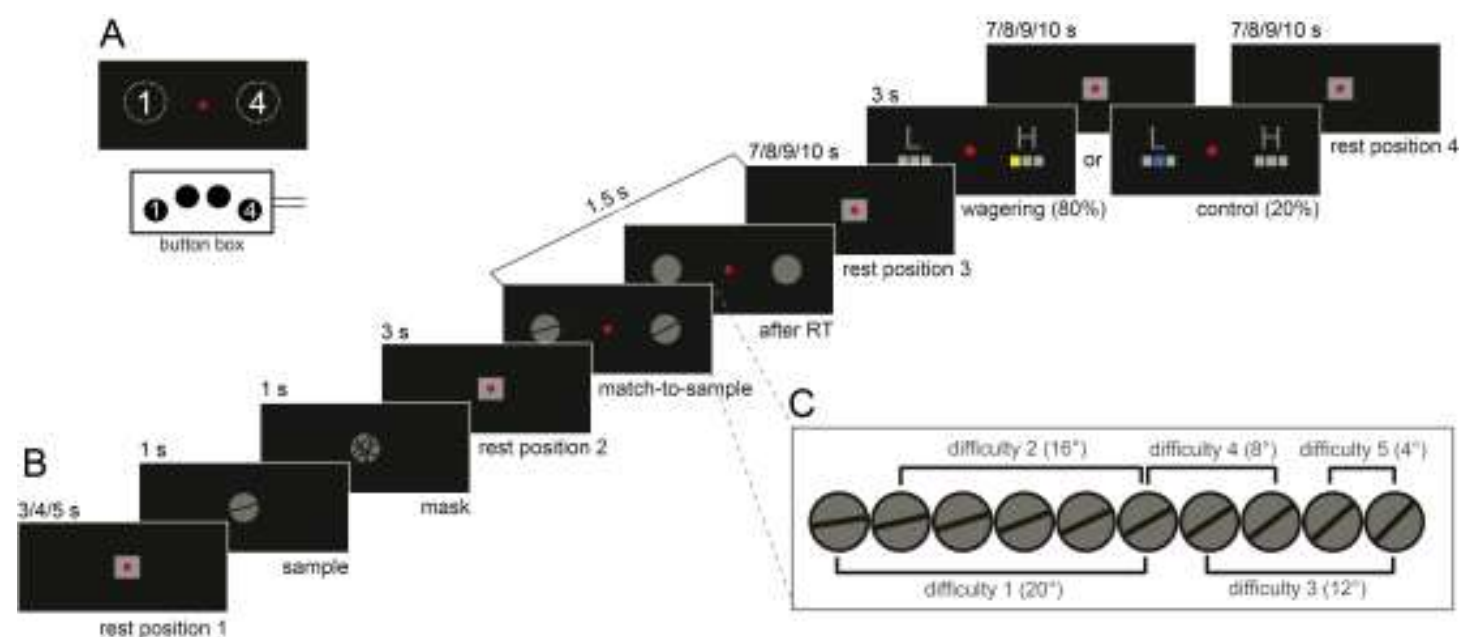

Figure 1. Task design. (A) In the two response periods (match-to-sample and PDW/control), subjects used the button and hand in the same side of the screen as the intended option. (B) A bright red spot and a gray filled square at the center of the screen indicated that subjects adopted in the rest position (gaze fixation inside the fixation window and left and right thumbs on the buttons 1 and 4 , respectively). After staying in the rest position for 3/4/5 s (rest position period 1), the sample was presented for $1 \mathrm{~s}$, followed by a mask presentation (also $1 \mathrm{~s}$ ). After the mask, subjects maintained the rest position for $3 \mathrm{~s}$ (rest position period 2) before the match-to-sample. Subjects used the buttons 1 or 4 to select the image they believed was the match. They had up to $1.5 \mathrm{~s}$ to respond. After the response, in order to keep the same visual stimulation across all trials, the bars disappeared but the circles remained until the completion of $1.5 \mathrm{~s}$ from match-to-sample presentation. The rest position period 3 (7/8/9/10 s) separated match-to-sample from PDW/control event. During PDW ( $80 \%$ of the trials), the letters $\mathrm{H}$ (high) and L (low) were presented on the right and on the left of the screen above gray squares representing the wagers. As instructed to and practiced by the subjects, the wager categories had always the same position below the letter they were associated to (wager categories 1, 2 and 3 below "L", and wager categories 4, 5 and 6 below "H"), but high and low position varied randomly across trials. After subjects selected to wager high or low, a yellow square cursor appeared randomly above one of the three gray squares. Subjects used the same button to choose a specific wager. The selection always moved from center-out and was restricted up to two movements. It means that the cursor could 
wrap around from out to center, but could not return to the initial position. The selection was visually confirmed by a green square appearing above the selected wager for $0.3 \mathrm{~s}$. The control task was similar to PDW, except that subjects were instructed to select high or low and then a specific "wager" according to the instruction given by a blue square that appeared above one of the wagers. After PDW/control, subjects maintained the rest position for 7/8/9/10 s (rest position period 4) before the intertrial interval ( $2 \mathrm{~s})$. (C) Five difficulty levels were created by different orientation contrasts between the match and the non-match options (linearly from $4^{\circ}$ to $20^{\circ}$ ).

The rest position periods 1,3 and 4 were jittered to decrease the prediction of the onset of following events and to improve the estimation of the regressors used in our fMRI analyses. The rest position period 2 was not jittered to keep the same time interval (and therefore the same memory requirements) between sample presentation and match-to-sample across different trials. We chose the jittered rest position periods so they fitted our task design and incorporated the smallest total amount of unexplained variance in our models. This variance was estimated using $3 \mathrm{dDeconvolve}$ function (nodata option, AFNI) as described by Filimon et al. (2013). The selected series of rest position periods were randomized across different runs.

\subsection{Time-course of experiment: practice and experimental sessions}

Subjects performed one practice session (60 minutes) outside the scanner to become familiar with the task contingencies, especially the long resting position periods required by the slow event-related design. They completed the practice session and the three scanning sessions on different days. The scanning sessions included one anatomical scan and four functional runs of 15 minutes. Feedbacks about the overall earnings were presented every scanning session after the second and fourth runs. During the anatomical run of the first session, subjects performed seven practice trials to become familiar with the in-bore setup.

\subsection{Behavioral data analysis}

The behavioral data were analyzed for performance, wagering, reaction time and sensitivities on perceptual and metacognitive decisions. The analysis was performed using MATLAB (Mathworks). When required, post-hoc tests were performed and corrected using Bonferroni correction. 
The performance associated to a certain wager was calculated dividing the number of correct trials by all trials this wager was assigned to. In order to test if the performance across different wagers varied because of the assignment of those wagers to correct or incorrect trials, we also calculated wager-specific proportions of correct trials ("number of correct trials each wager was assigned to" divided by "all correct trials") and wager-specific proportions of incorrect trials ("number of incorrect trials each wager was assigned to" divided by "all incorrect trials"). Both measures were used to create our fMRI-related models as described in fMRI Data Analysis.

The task contained two manual response periods: match-to-sample and wagering. The match-to-sample reaction time (named RT1) represented the time to select a possible match; and the wagering reaction time (named RT2) represented the time to select between wagering high or low. Since faster reaction times are associated with increased certainty (Festinger, 1943; Kiani et al., 2014) and because we assumed that subjects used wagers to maximize their earnings and, as consequence, implicitly reported their certainty; we also calculated RT1 and RT2 for each wager (wagerspecific RT1 and wager-specific RT2, respectively).

Lastly, in order to obtain a comparable and unbiased measure of perceptual decision and metacognitive sensitivities, we used Maniscalco and Lau (2012) code provided at: http://www.columbia.edu/ bsm2105/type2sdt/archive/index.html to calculate d' (perceptual decision sensitivity) and meta-d' (metacognitive decision sensitivity). Meta-d' is calculated using the same parameters of a Signal Detection Theory (SDT) model used for perceptual decisions, thus d' and meta-d' are in the same scale. The code estimates the value of d' that would have been required to produce the observed hits and false alarms of the metacognitive decisions. In our task, metacognitive efficiency (meta-d'/d') demonstrates how much the sensitivity associated to wagering was improved (meta-d'>d') or worsened (meta-d' $<$ d') compared to the sensitivity associated to perceptual decisions. According to Moreira et al. (2016), metacognitive efficiency can also reveal how much post-decisional evidence subjects were able to accumulate and use for their metacognitive judgments. 


\subsection{MRI Data Acquisition and preprocessing}

Subjects were scanned using a Siemens Trio 3T scanner and a Siemens 32-channel head coil. High-resolution structural scan (T1-weighted MPRAGE volume, $1 \times 1 \times 1$ mm, TR $2300 \mathrm{~ms}$, TE $3.97 \mathrm{~ms}$, 192 slices, flip angle: $9^{\circ}$ ) was acquired for anatomical localization. Functional images were acquired with EPI sequences $(3 \times 3 \times 3 \mathrm{~mm}$, TR $1000 \mathrm{~ms}$, TE 33ms, 36 transverse slices, multiband-factor 3, $0.15 \mathrm{~mm}$ gap between adjacent slices). The anatomical T1-weighted scans were processed in BrainVoyager QX 2.4 (Brain Innovation). The anatomical images from sessions 2 and 3 were coregistered to the first session in the commissure-posterior commissure (AC-PC) plane and then transformed from AC-PC into Talairach space. Each functional run consisted of 904 volumes. The first four volumes of each run were excluded from functional analyses but were used for coregistration. EPI series from each run were preprocessed in NeuroElf 1.0 (Jochen Weber, www.neuroelf.net) using slice time correction, linear trend removal, and a high-pass temporal filter with three cycles per 10 min run cut-off. The first functional volume in the session was aligned to the original anatomical T1-weighted scan using rigid body transformations. 3D motion correction with 6 degrees of freedom was done by registering all EPI volumes to the first volume of the first run in the session. 3D volume time courses were computed in Talairach space using the anatomical AC-PC and Talairach transformation parameters (voxel size $3 \times 3 \times 3 \mathrm{~mm})$. Additional spatial smoothing $(6 \times 6 \times 6 \mathrm{~mm})$ was applied to the $3 \mathrm{D}$ volume time courses.

\section{9 fMRI Data Analysis}

Several GLMs were used to estimate the BOLD response associated with specific components of the task. The GLMs were performed only for the voxels encompassed in a mask based on the Colin brain (Holmes et al. 1998), transformed into the Talairach space. The regressors were convolved with a canonical hemodynamic response function (time-to-positive-peak: $5 \mathrm{~s}$, time-to-negative-peak: $15 \mathrm{~s}$ ).

We modeled five events of main interest: fixation, sample presentation, match-tosample, wagering and control (see Supplementary Table 1 for information about the onset and duration of each regressor). In addition to the main regressors, all GLMs contained eight regressors of no interest: (1) trial abortion due to inappropriate button use (e.g. releasing the buttons during rest position periods), (2) trial abortion because 
of breaking gaze fixation, and (3-8) six head motion regressors extracted during fMRI data preprocessing. When an event aborted the continuation of the trial, the previous trial events were still modeled and used in our estimations.

Three of the main events: fixation, sample and control were modeled using nonparametric regressors. The events: match-to-sample and wagering were modeled using parametric regressors in order to search for areas whose activity varied according to specific patterns associated with performance per difficulty level (matchto-sample event) or associated with certainty readouts (wagering event). Since we assume that there are three possible certainty readouts (certainty of being correct, certainty of being incorrect and bi-directional certainty), we created one wagering parametric regressor for each certainty readout and we entered them in three separate GLMs.

The regressors expansions were selected by fitting the behavioral data (performance per difficulty level or wager-specific proportions of correct and incorrect trials) to different functions (linear, quadratic and exponential) and, afterwards, choosing the best fitting function using the Akaike information criterion (AIC) comparison (Burnham \& Anderson, 2004; Field, 2009).

Performance per difficulty level was best described by the quadratic function (AIC = $513)$ compared to linear $(\mathrm{AIC}=-503)$ and exponential $(\mathrm{AIC}=-495)$ functions (see Supplementary Fig. 1). The increase in wager-specific proportions of correct trials was best described by the exponential model (AIC $=593$ ) compared to quadratic (AIC $=614)$ and linear $(\mathrm{AIC}=633)$ models $($ Fig. $2 \mathrm{~A})$. The same occurred for the decrease in wager-specific proportions of incorrect trials, whose AIC values were 578, 591 and 614 for exponential, quadratic and linear functions, respectively (Fig. 2B). 
A

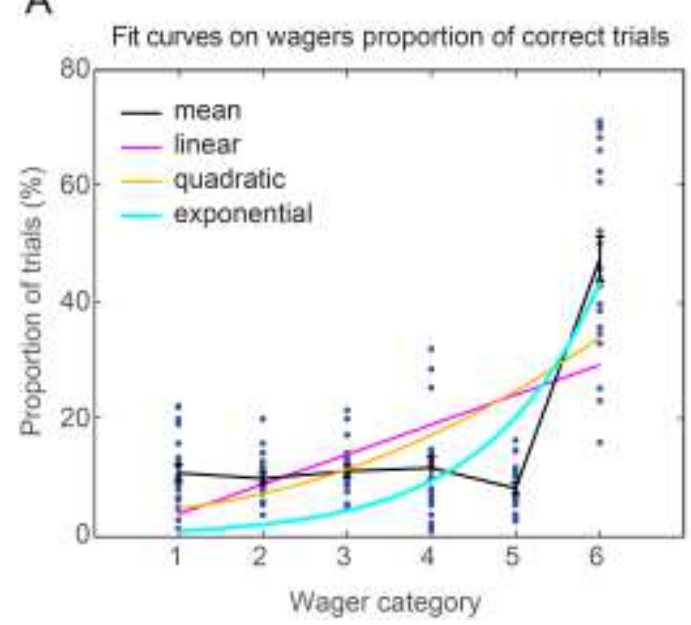

B

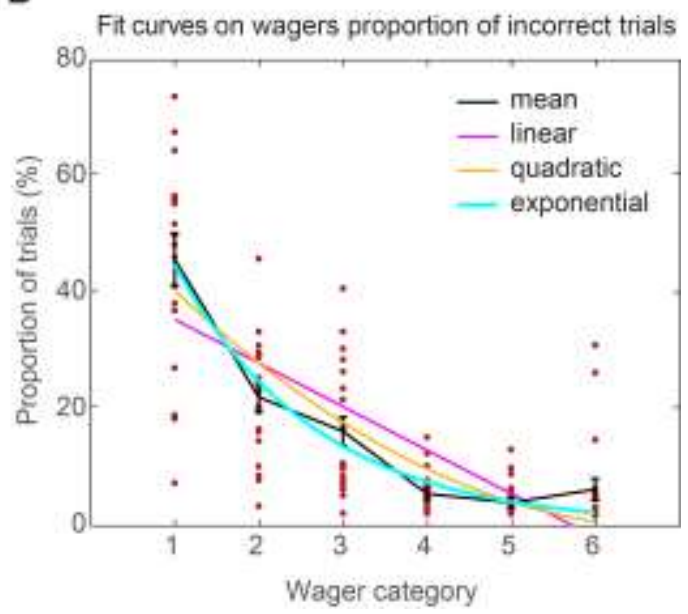

Figure 2. Fit curves (linear, quadratic and exponential), subjects' means (dots) and group means and standard errors (black curve) related to the wager-specific proportions of (A) correct trials and (B) incorrect trials.

We calculated the weights of the match-to-sample parametric regressor using the quadratic function fitted to subjects' performance per difficulty level. This parametric regressor was used to model the match-to-sample event of the three GLMs: certainty of being correct, certainty of being incorrect and bi-directional certainty. Therefore, the only difference between these GLMs (or models) was the regressor for the wagering event. The weights for the wagering parametric regressor of the certainty of being correct model (Fig. 3A) were calculated using the exponential function fitted to the wager-specific proportions of correct trials (Fig. 2A). Conversely, we used the exponential function fitted to the wager-specific proportions of incorrect trials (Fig. 2B) to calculate the weights of the wagering parametric regressor of the certainty of being incorrect model (Fig. 3B).

According to its own definition, the bi-directional certainty model should contain a wagering parametric regressor with two branches of opposite directions. One branch associated with low wagers, and another branch associated with high wagers. We calculated the weights associated with low wagers using the exponential function fitted to the wager-specific proportions of incorrect trials, while the weights associated with high wagers were calculated using the exponential function fitted to the wager-specific proportions of correct trials (Fig. 3C; see Supplementary information for details about the calculation of the parametric weights). 

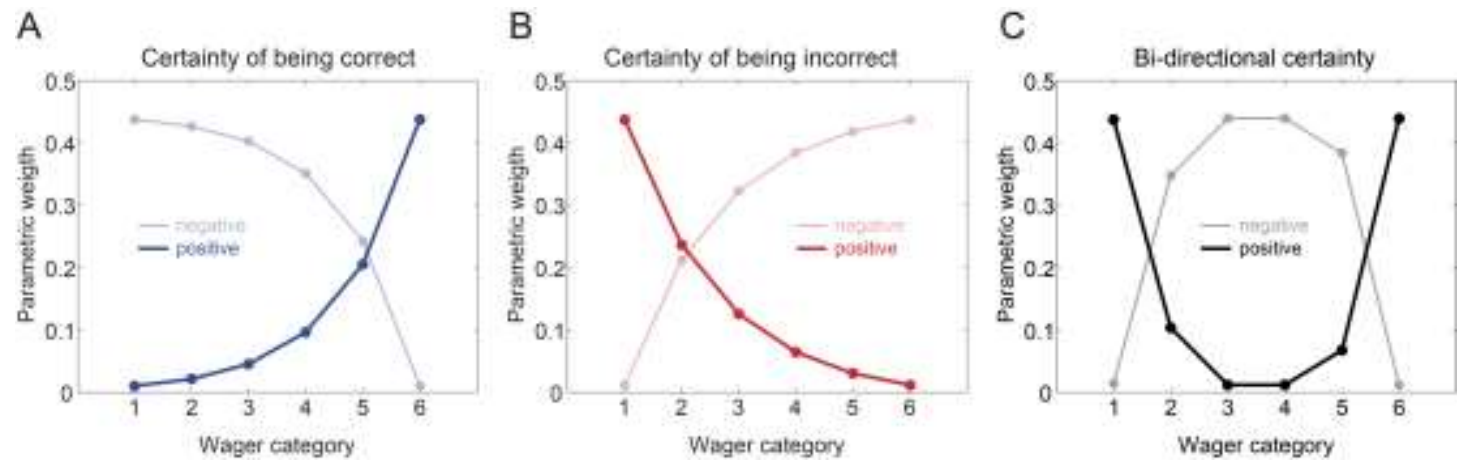

Figure 3. Parametric weights used for the wagering predictor in the models: (A) Certainty of being correct, (B) Certainty of being incorrect and (C) Bi-directional certainty, for positive (dark curves and dots) and negative (light curves and dots) activation patterns.

The relationship between the different modeled events and brain activity was analyzed for each subject. Beta estimates were tested against zero (t-test) to assess group level significance. All reported brain activations survived FDR correction (FDR $<0.05$ at RFX level).

\subsubsection{Exponential relationship between wagering and brain activation}

Most studies used linear modulations in their certainty-related analyses to map brain areas whose activity varied with certainty of being correct, but so far none of them provided arguments for choosing such modulation. Hebart et al. (2014), for example, provide a detailed description about how they calculated a proxy of DV from perceptual and metacognitive reports, and used these measures in their fMRI analyses; but the authors did not justify the use of a linear relationship between brain activity and the calculated proxy.

In the present study, we suggest that the large difference between the proportion of incorrect trials of wager category 1 and the other wagers and, especially, between the proportion of correct trials of wager category 6 and the other wagers, indicate that subjects were using wagers non-linearly as they were attempting to maximize their earnings, thus implicitly reporting their certainties non-linearly. Supporting this notion, not only the wager-specific proportions of correct and incorrect trials were best described by exponential functions, but this function also described best subjects' 
criteria to select the wagers (see Supplementary Fig. 2). On top of that, we highly profited from using exponential functions in our GLMs because, by attributing very different variances to low and high wagers, we created unidirectional models which were able to differentiate certainty of being correct from certainty of being incorrect. For example, while the BOLD signal variance in the certainty of being correct model is mainly attributed to differences between high wagers, the BOLD signal corresponding to the selection of high wagers should vary minimally in the certainty of being incorrect model (comparison between Figure $3 \mathrm{~A}$ and Figure 3B). Nonetheless, we also tested a linear model and presented evidence that the brain activity related to certainty of being correct is better described by the exponential model, matching behavioral expectations (see Supplementary Information, Supplementary Fig. 3 and Supplementary Fig. 4).

We are aware that wager-specific proportions of correct and incorrect trials do not reflect, on their own, subjects' readouts of certainty of being correct and certainty of being incorrect (Moreira et al., 2016). Thus, before the practice and experimental sessions of the present fMRI experiment, all subjects also performed the task used in Moreira et al. (2016) to confirm that, on average, they were able to wager adaptively by significantly reading out both certainty directions (see Supplementary Fig. 5). This task contained a baseline condition (pre-decision wagering) that allowed us to isolate and quantify PDW trial-specific readouts for each certainty direction independently.

\subsubsection{Additional post-hoc tests for regions of interest}

In order to further test the group level results of our parametric-GLMs, we created a non-parametric-GLM that modeled each difficulty level and each wager using separate regressors. Therefore, instead of using one parametric regressor for the match-to-sample event and one parametric regressor for the wagering event as in the parametric-GLMs, we used the non-parametric-GLM to estimated separate beta values which were submitted to a third level analysis that aimed to further test if the activity patterns matched to the patterns predicted by the models. This extra level of analysis (consisting of additional post-hoc tests) also aimed at preventing that more than one certainty-related activation pattern were assigned to the same brain region. For example, identify brain regions included in both certainty of being correct and bi- 
directional certainty maps because the activity associated with high wagers was enough to generate beta estimates which survived second level analysis thresholds, even if the activity pattern associated with low wagers and predicted by the bidirectional certainty model was not present.

Although the non-parametric-GLM was performed for all the voxels encompassed in our brain mask (see section 2.9), since we were interested in further test the results of our parametric-models ("basic maps"), we only extracted the beta estimates from activation clusters of voxels located with these models (i.e. regions of interest). These clusters should have more than 20 voxels and should have survived FDR correction $($ FDR $<0.05)$. Moreover, the extraction of beta estimates was restricted to a sphere of two functional voxels radius around the cluster peak-voxel (33 functional voxels in total) in order to distinguish, despite the low spatial resolution $(1.5 \mathrm{~cm}$ diameter spheres), different regions in the same brain area.

The additional post-hoc tests (paired t-tests) were map-specific because each parametric regressor predicted a specific activity pattern, therefore: (1) clusters from the certainty of being correct map should present significant differences between the beta values of wagers 4 and $6(\mathrm{p}<0.05)$. At the same time, differences between the wagers 1 and 3 in the opposite direction of the difference between wagers 4 and 6 should not be significant ( $>0.05$ ) in order to guarantee unidirectionality (Fig. 4A); (2) Clusters from the certainty of being incorrect map should present significant differences between the beta values of wagers 1 and $3(\mathrm{p}<0.05)$. Concomitantly, differences between the wagers 4 and 6 in the opposite direction of the difference between wagers 1 and 3 should not be significant ( $p>0.05$; Fig. 4B); (3) Clusters from the bi-directional certainty map should present significant differences between the beta values of wagers 1 and $3(\mathrm{p}<0.05)$ and, in the opposite direction, between the wagers 6 and 4 (p<0.05; Fig. 4C). 
A

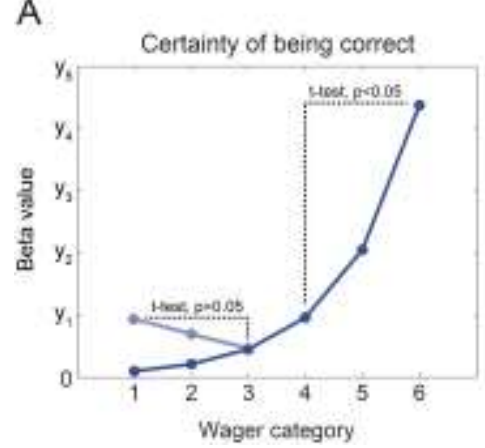

B

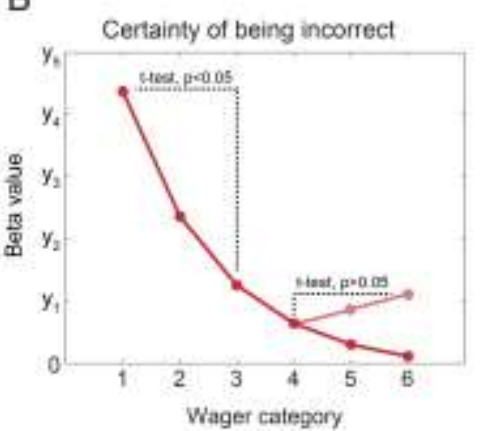

C

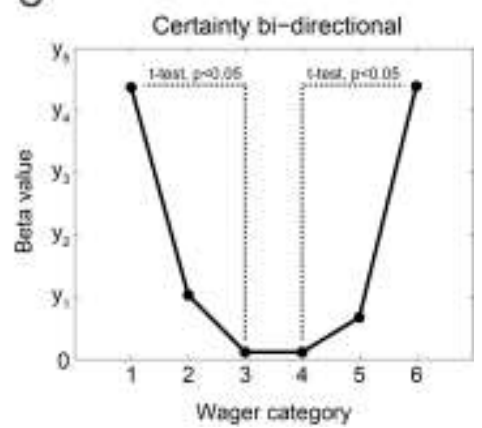

Figure 4. Additional post-hoc tests applied to the beta values extracted from clusters of the basic maps. (A) Certainty of being correct. (B) Certainty of being incorrect. (C) Bi-directional certainty. Only one activation pattern (positive or negative) was exemplified for each model.

Clusters located with the match-to-sample parametric regressor should present significant differences $(\mathrm{p}<0.05)$ between the beta values of difficulty levels 1 and 5 . All maps presented in the Results section contain only the voxels that survived the additional post-hoc tests. Basic maps are shown in the Supplementary Figures 6, 7, 8 and 9.

\section{Results}

20 human subjects were asked to carry out a perceptual decision (delayed match-tosample, DMTS) of varying difficulty followed by post-decision wagering (PDW) inside an MRI scanner.

\subsection{Behavioral results}

We performed one-way ANOVA to test basic task-related behaviors. As expected, the performance in perceptual decisions decreased on harder trials $\left(\mathrm{F}_{4,76}=113.842\right.$, $\mathrm{p}<$ 0.0001; Supplementary Fig. 1) and on lower wagers $\left(\mathrm{F}_{5,95}=61.646, \mathrm{p}<0.0001\right)$. Wager-specific proportions of correct trials increased towards the highest wager $\left(\mathrm{F}_{5,95}\right.$ $=38.691, \mathrm{p}<0.0001$; Fig. 3A). Post-hoc tests showed that the wager-specific proportions of correct trials increased only at wager category $6(\mathrm{p}<0.0001)$. No significant differences were found for the comparisons between wagers 1 to 5 ( $>0.05)$. Wager-specific proportions of incorrect trials increased towards the lowest 
wager $\left(F_{5,95}=40.507, p<0.0001\right.$; Fig. 3B $)$. Post-hoc tests showed that, while the proportions on low wagers (1,2 and 3) were significantly different between each other and from all high wagers $(\mathrm{p}<0.05)$, the proportion of incorrect trials of high wagers did not vary $(\mathrm{p}>0.05)$. These results indicate that the performance on high wagers varied only because of the different wager-specific proportions of correct trials, while the performance on low wagers varied only because of different wagerspecific proportions of incorrect trials.

According to our analyses for reaction times, $\mathrm{RT} 1$ increased on harder trials $\left(\mathrm{F}_{4,76}=\right.$ 30.591, $\mathrm{p}<0.0001$; Fig 4A). This result suggests that, although the stimuli were static, subjects might have delayed harder decisions to acquire more perceptual evidence. For the RT2, our previous study (Moreira et al., 2016) showed that wagerspecific RT2 might reveal the use of the wagering scale to report bi-directional certainty. In the present study, RT2 differed among wagers $\left(F_{5,95}=9.831, p<0.0001\right)$. The post-hoc test showed that they were shorter in the wager categories 1 and 6 than in the middle wagers (categories 3 and 4; p<0.05), generating an inverted U-shape function of wager-specific RT2 (Fig. 4B). Therefore we associate, as in Moreira et al. (2016), fast RT2 in the wager category 1 with increased certainty of being incorrect and fast RT2 in the wager category 6 with increased certainty of being correct. We also calculated wager-specific RT1 to test if the reaction time bi-directionality was already present at the moment subjects performed perceptual decisions, and could determine their wager selection. Since certainty of being incorrect is intrinsically dependent on post-decisional evidence (Moreira et al., 2016), we should not find fast RT1 in the lowest wager category compared to the middle ones. As expected, wagerspecific RT1 was unidirectional, decreasing towards the highest wager $\left(\mathrm{F}_{5,95}=15.998\right.$, $\mathrm{p}<0.0001$; Fig. 4C).
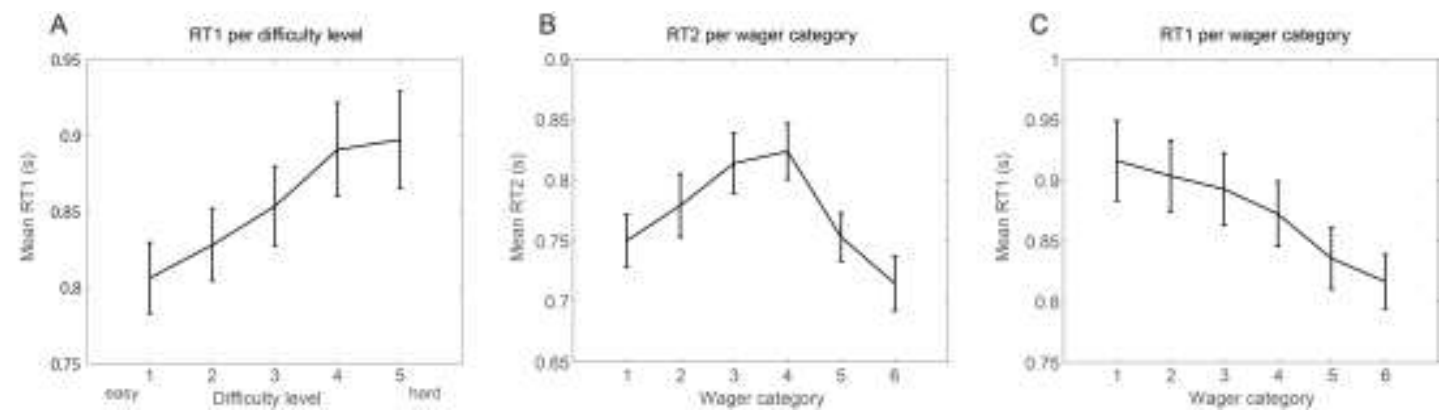
Figure 4. (A) Means and standard errors of $\mathrm{RT}_{1}$ for each difficulty level. (B) Means and standard errors of wager-specific RT2. (C) Means and standard errors of wager-specific RT1. All measurements were averaged across subjects.

We used a paired t-test to compare the sensitivities on perceptual (d') and metacognitive (meta-d') decisions. Meta-d' was higher than d' $\left(t_{19}=-4.457, p<0.001\right)$ indicating that, on average, subjects performed better on metacognitive than on perceptual decisions (meta-d'>d'; Fig. 5).
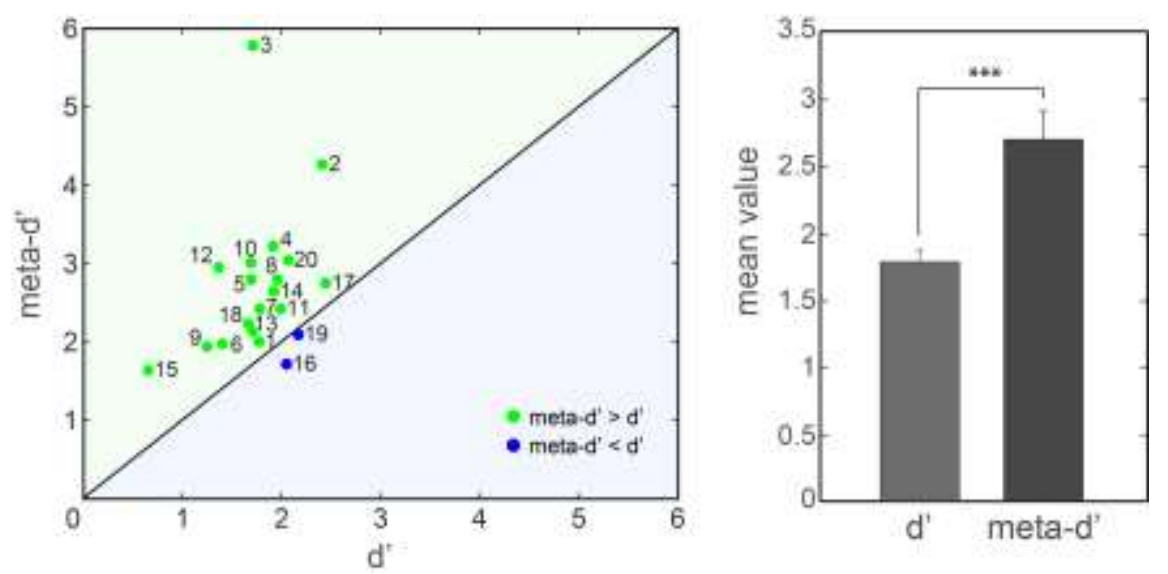

Figure 5. (A) Metacognitive sensitivity (meta-d') plotted as a function of perceptual decision sensitivity (d'). 18 subjects with meta-d'>d' (high metacognitive efficiency subjects) fall above the diagonal (green area), and 2 subjects with meta-d' $<$ ' (low metacognitive efficiency subjects) fall below the diagonal (blue area). (B) Means and standard errors of d' and meta-d' values $(* * * p<0.001)$.

\section{2 fMRI results}

The three parametric-GLMs contained the parametric regressor for the match-tosample event, but their parametric regressor for the wagering event were different in order to locate brain areas whose activity varied according to specific patterns related to one of the three certainty types: bi-directional certainty, certainty of being correct or certainty of being incorrect. 


\subsubsection{Match-to-sample event}

The activity on 37 clusters of voxels varied parametrically according to the five difficulty levels of the perceptual decision (match-to-sample event; see Supplementary Table 2 for more information about the clusters). These clusters were located mostly in cortical areas (Fig. 6).

\section{Left hemisphere}

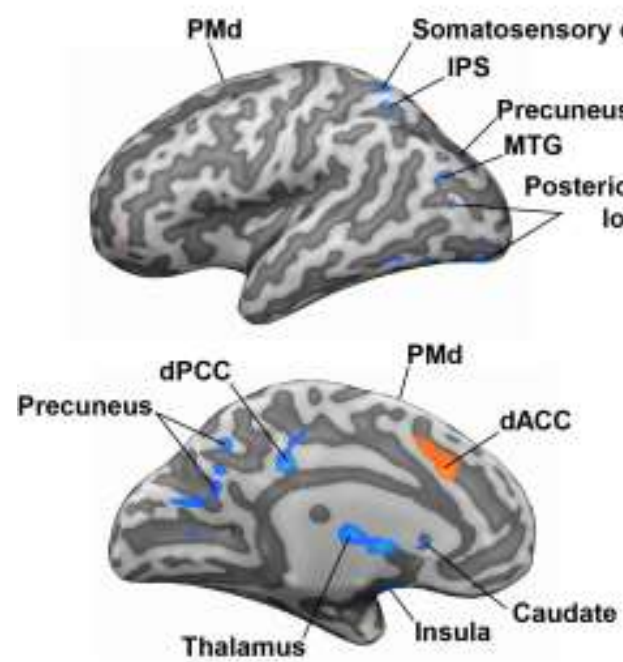

Right hemisphere
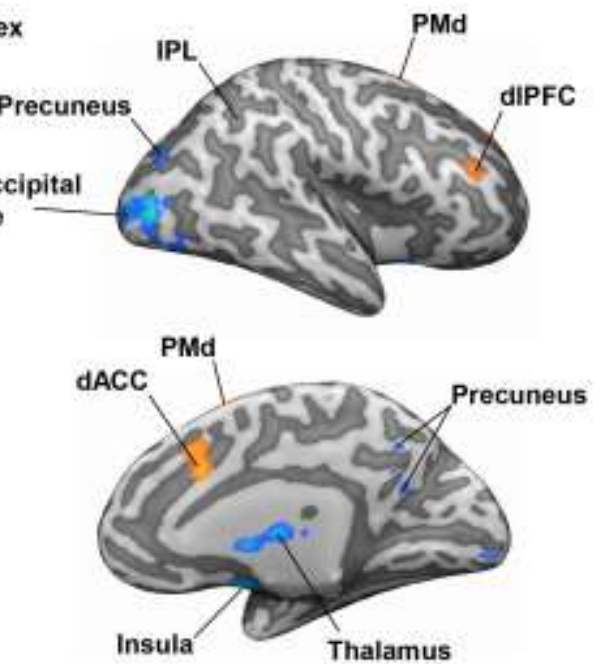

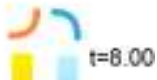
$p<0.01$
fan 0.05 )

Figure 6. Map on inflated brain of cortical areas whose activity varied parametrically according to the five difficulty levels of the perceptual decision (match-to-sample event) and survived additional posthoc tests.

The additional post-hoc tests (see Methods) provided further support in matching the activity pattern present in the intersubject averages of wager-specific beta values with the pattern predicted by the parametric models (Fig. 7). 

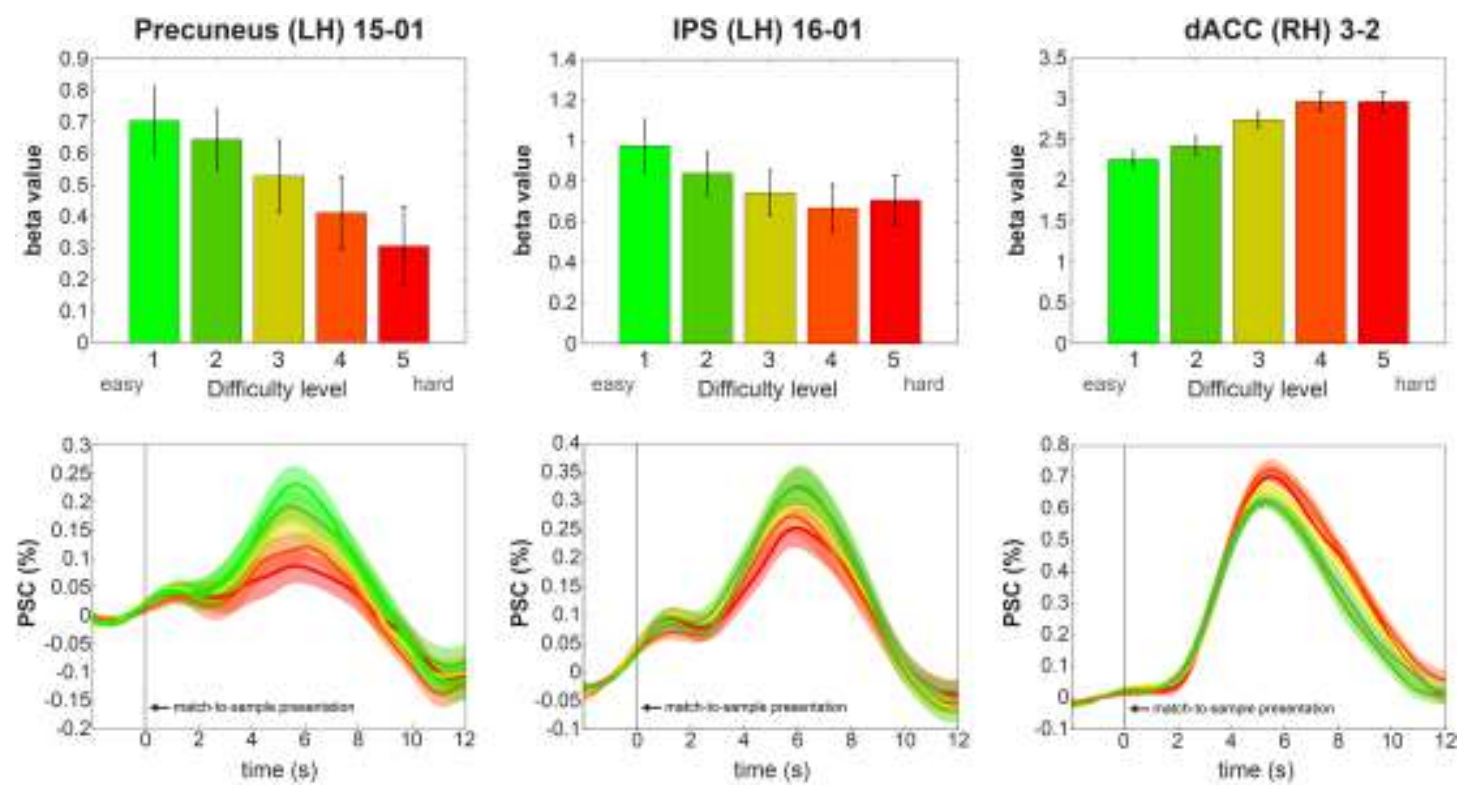

Figure 7. Beta values and averaged time courses of three areas: precuneus (left hemisphere), intraparietal sulcus (IPS, left hemisphere) and dorsal anterior cingulate cortex (dAAC, right hemisphere). In both rows, difficulty varies from the easiest level (level 1, light green) to the hardest level (level 5, red). In the first row, beta value means and standard errors for each of the five difficulty levels non-parametric regressors. In the second row, averaged time courses in percent signal change (PSC) for each of the five difficulty levels. Averaged time courses baseline was set from -2 to $0 \mathrm{~s}$ in relation to the match-to-sample presentation.

Although it is not shown in the inflated brain, the ventral striatum in both hemispheres (Talairach coordinates: $\mathrm{x}= \pm 8 \mathrm{~mm}, \mathrm{y}=6 \mathrm{~mm}$ and $\mathrm{z}=0 \mathrm{~mm}$ ), described by Herbart et al. (2014) as reflecting the degree of perceptual confidence, was also present in the match-to-sample map (see Supplementary Fig. 10).

\subsubsection{Wagering event}

The bi-directional certainty map represents our main results because it is the first empirical demonstration that brain areas can encode both certainty directions (certainty of being correct and certainty of being incorrect; Fig. 8). From the 125 clusters located through the FDR corrected contrast between the wagering parametric regressor (bi-directional function) and the baseline, 47 clusters survived our additional post-hoc tests (Fig. 8). 
Left hemisphere

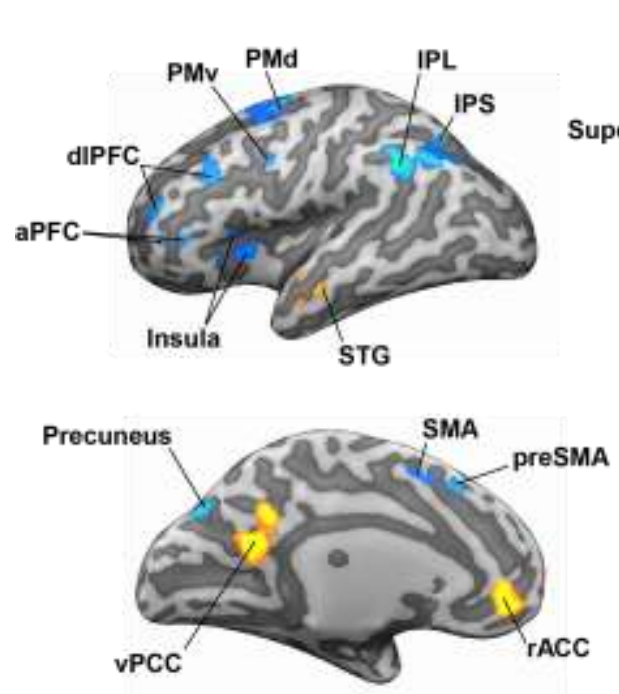

Right hemisphere
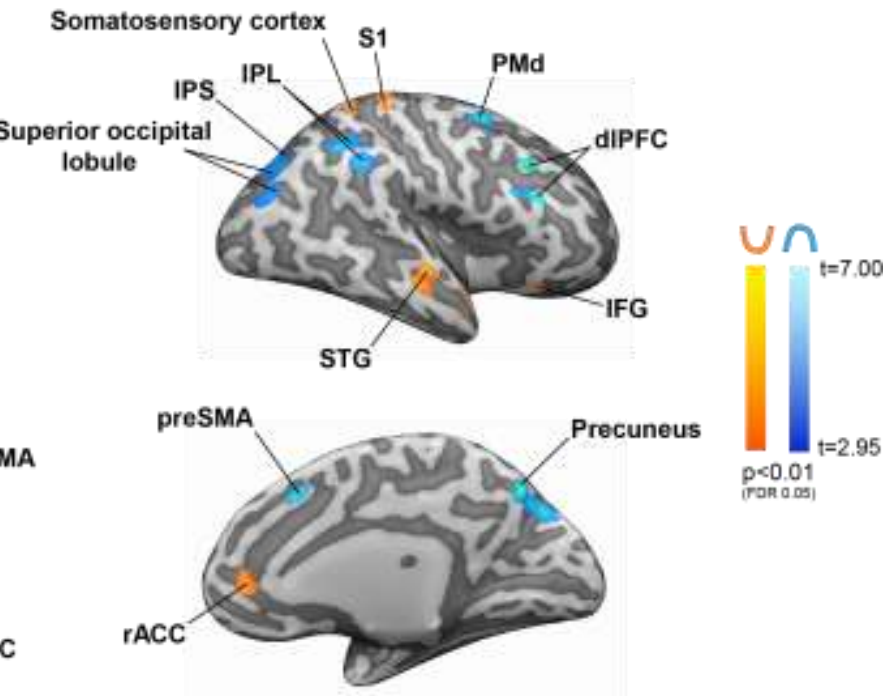

Figure 8. Map on inflated brain of cortical areas whose activity varied parametrically according to the bi-directional certainty regressor of the wagering event and survived additional post-hoc tests.

These additional post-hoc tests further supported the (inverted)-U-shape function of wager-specific brain activity of the clusters present in the bi-directional certainty model (see Supplementary Table 3) as exemplified in the Figure 9.
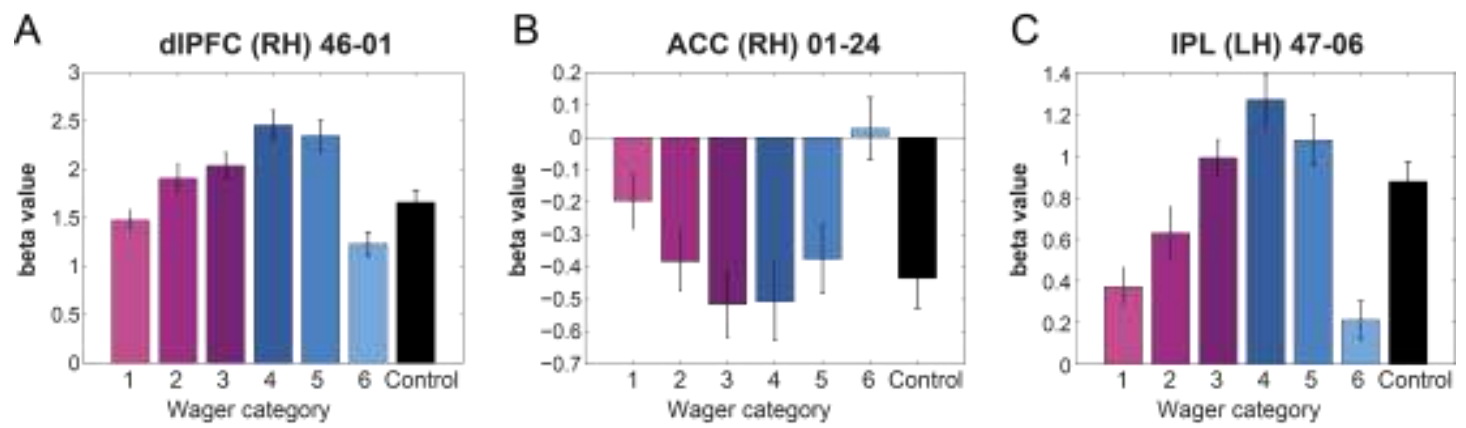

Figure 9. Beta value means and standard errors of wagers and control non-parametric regressors of three example areas located with the bi-directional certainty model. (A) dorsolateral prefrontal cortex (dlPFC, right hemisphere), (B) anterior cingulate cortex (ACC, right hemisphere) and (C) intraparietal lobule (IPL, left hemisphere). Wagers are presented from the smallest stack (category 1, pink) to the largest stack (category 6, light blue). The activity related to control trials (instructed wagering) is represented in the black bar.

Brain areas encoding certainty in a bi-directional way were identified mainly in the frontal, parietal and cingulate cortices. Many of them were previously related to 
certainty of being correct and certainty of being incorrect studies, suggesting their flexibility in encoding information according to the task requirements.

The certainty of being correct map included 69 clusters of voxels with exponentially increasing or decreasing activity as subjects wagered higher. 41 of these clusters survived the additional post-hoc tests. Most of them were located in the brain cortex (Fig. 10).

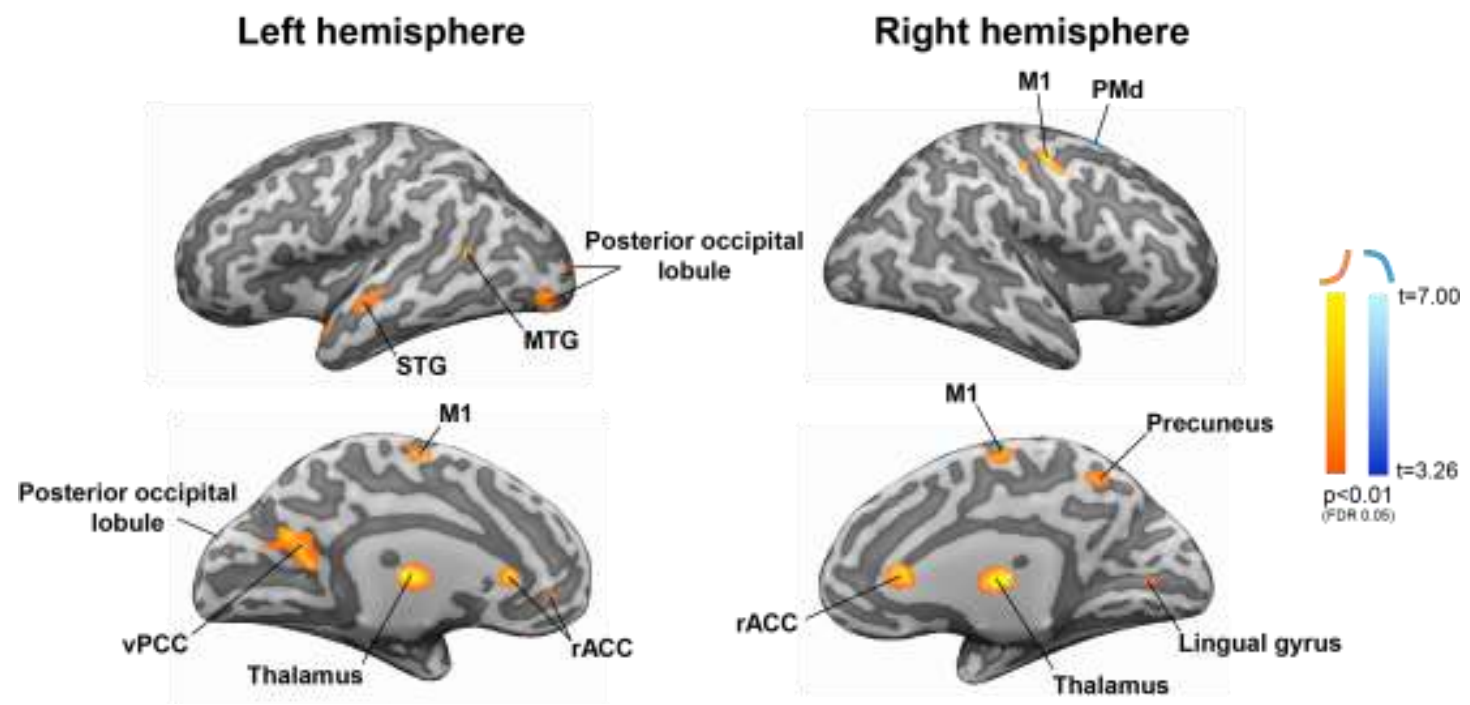

Figure 10. Map on inflated brain of cortical areas whose activity varied parametrically according to the certainty of being correct regressor of the wagering event and survived additional post-hoc tests.

The post-hoc tests provided further support that these clusters of voxels (see Supplementary Table 4) presented an exponential and unidirectional function of wager-specific brain activity (Fig. 11).
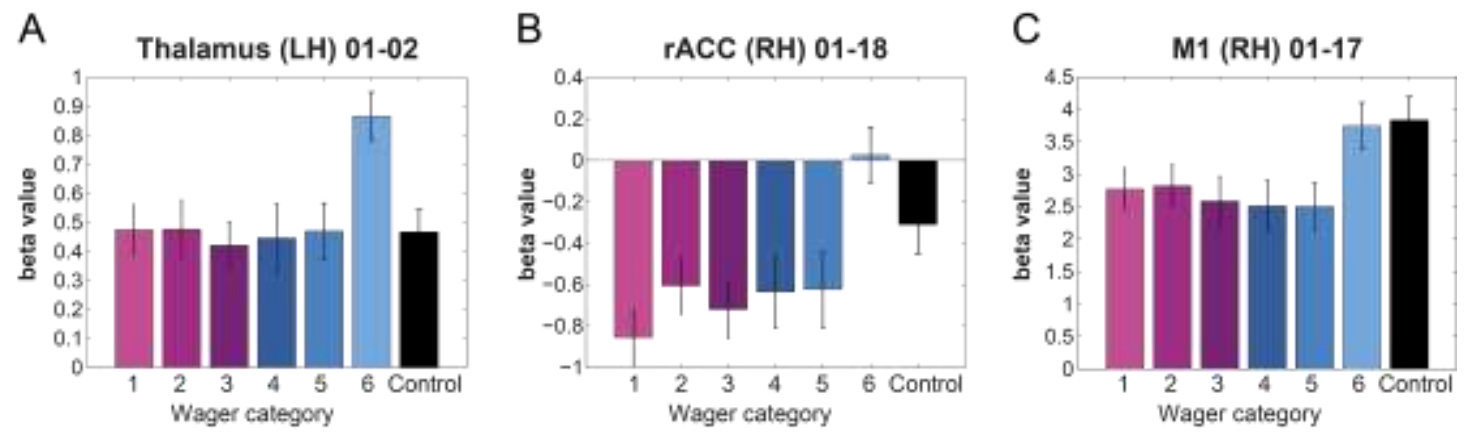

Figure 11. Beta value means and standard errors of wagers and control non-parametric regressors of three example areas located with the certainty of being correct model. (A) thalamus (left hemisphere), 
(B) rostral anterior cingulate cortex (rAAC, right hemisphere) and (C) primary motor cortex (M1, right hemisphere). Wagers are presented from the smallest stack (category 1, pink) to the largest stack (category 6, light blue). The activity related to control trials (instructed wagering) is represented in the black bar.

Lastly, the brain activity increased or decreased exponentially as subjects wagered lower in 30 clusters of voxels, but only 7 of them (see Supplementary Table 5) survived the additional post-hoc tests (Fig. 12).
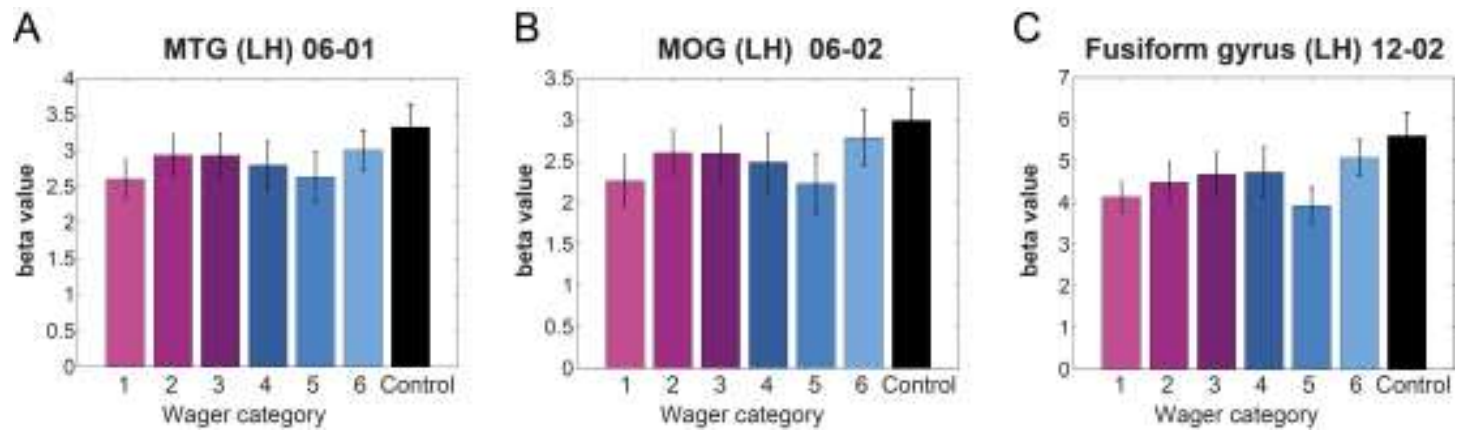

Figure 12. Beta value means and standard errors of wagers and control non-parametric regressors of three example areas located with the "Certainty of being incorrect" model. (A) middle temporal gyrus (MTG, left hemisphere), (B) middle occipital gyrus (MOG, left hemisphere) and (C) fusiform gyrus (right hemisphere). Wagers are presented from the smallest stack (category 1, pink) to the largest stack (category 6, light blue). The activity related to control trials (instructed wagering) is represented in the black bar.

Our post-hoc tests further supported the bi- or unidirectionality depending on the model and, by doing so, did not allow complete overlap of areas from different maps. Figure 13 illustrates the maps of the three models (independent on if the activity was positive or negative) and shows that only a portion of the ventral posterior cingulate cortex (vPCC) is present in both bi-directional certainty and certainty of being correct maps (Fig. 13). 
Left hemisphere

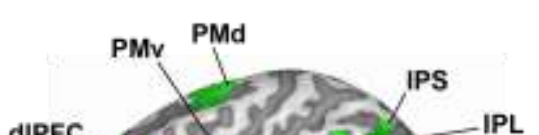

dIPFC

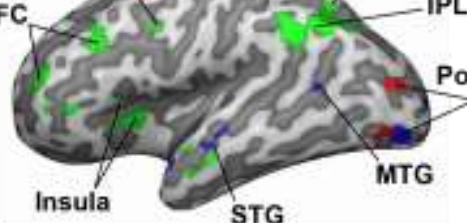

Insula
Right hemisphere

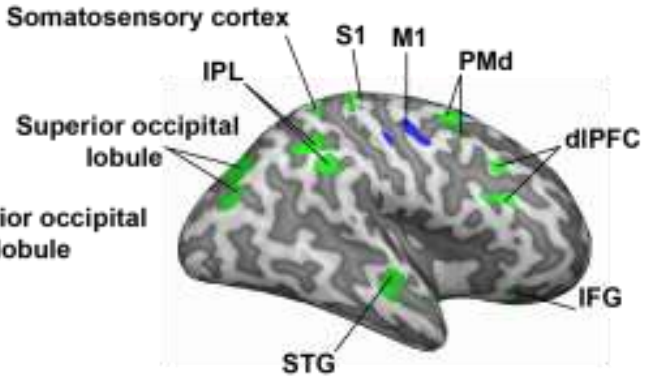

STG
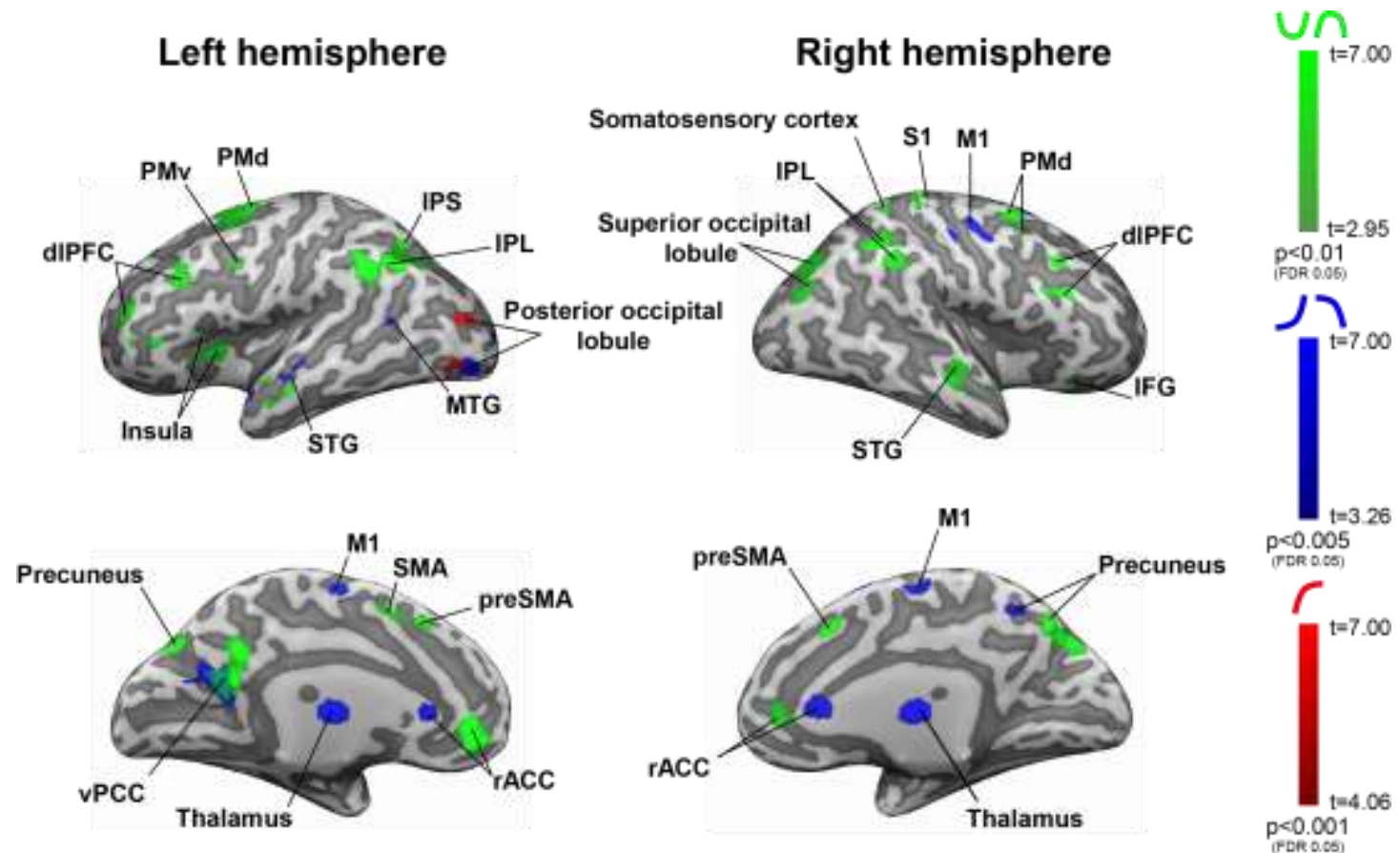

Figure 13. Maps on inflated brain of cortical areas whose activity varied parametrically according to the bi-directional certainty (green), certainty of being correct (blue) and certainty of being incorrect (red) regressors of the wagering event and survived additional post-hoc tests. vPCC is highlighted because it was present in two maps.

When only the overlapping voxels were considered, the activity pattern of vPCC did not survive the additional post-hoc test for bi-directionality ( $\mathrm{p}=0.08$ in the left branch; see Supplementary Fig. 11).

\subsubsection{Overlap between areas encoding difficulty levels and certainty}

No area encoded both: the difficulty levels during match-to-sample and bi-directional certainty or certainty of being incorrect during wagering, but two clusters of voxels (at the left thalamus - cluster 12-05 in the match-to-sample map and cluster 01-05 in the bi-directional certainty map; and at the right dorsal premotor cortex (PMd) cluster 01-01 in the match-to-sample map and cluster 28-01 in the bi-directional certainty map) encoded difficulty levels and certainty of being correct during the corresponding events of the task (Fig. 14). 
12-04 Thalamus (LH) $01-05$
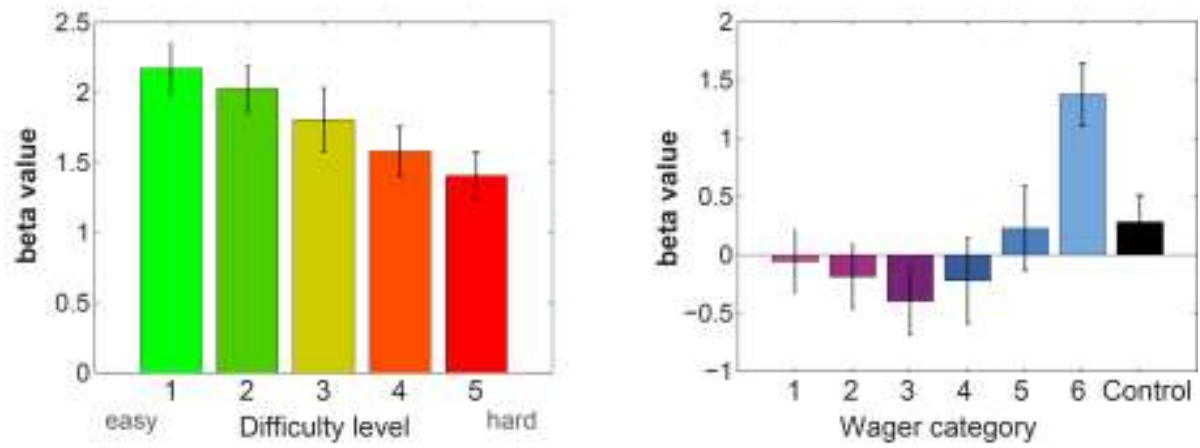

01-01 PMd (RH) 28-01
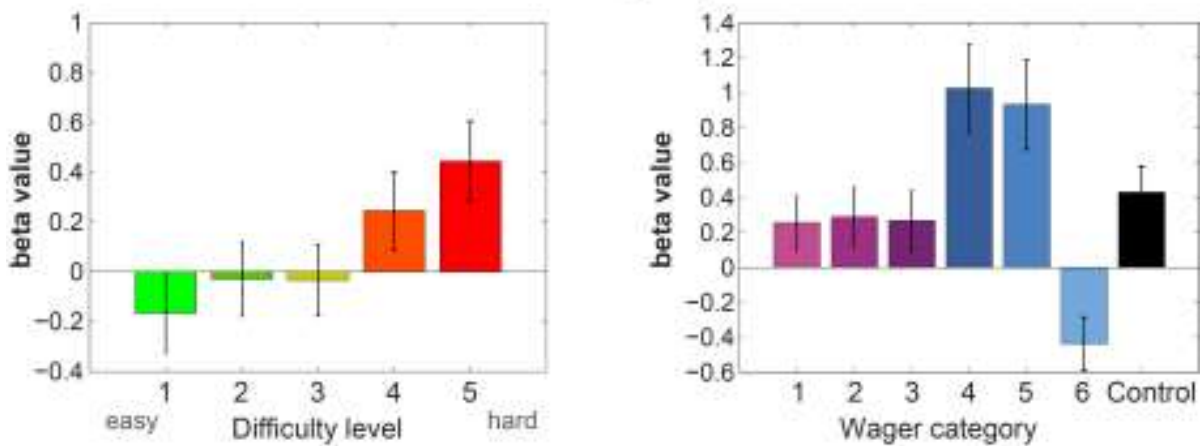

Figure 14. Beta value means and standard errors of non-parametric regressors for the different difficulty levels (left column) and for the different wager categories and control (right column) from the two areas whose activity varied parametrically as predicted by the corresponding parametric regressor of each event: match-to-sample and wagering (Certainty of being correct model). At the first row, information about the thalamus (left hemisphere). At the second row, information about the PMd (right hemisphere). Difficulty varies from the easiest level (level 1, light green) to the hardest level (level 5, red), and wagers are presented from the smallest stack (category 1, pink) to the largest stack (category 6, light blue). The activity related to control trials (instructed wagering) is represented in the black bar.

\subsubsection{The post-match-to-sample event}

Our task design allowed subjects to evaluate their perceptual decisions for several seconds (7 to $10 \mathrm{~s}$ during the rest position period 3, see Methods) before wagering. We created an additional model to test if areas encoding bi-directional certainty during the wagering period presented bi-directional pattern of activity already before the wagering stage. If yes, then these areas might be involved in the evaluation of post-decisional information that would eventually be used during wagering reports related to both certainty directions. 
In this model we used, instead of the match-to-sample event, a post-match-to-sample event that started at the end of the match-to-sample task $(1.5 \mathrm{~s}$ after the match-tosample presentation). We modeled the post-match-to-sample event using six nonparametric regressors that estimated the increase or decrease in the BOLD signal associated to the wagers subjects selected after the rest position period 3. Next, we extracted the beta values of the post-match-to-sample event for the clusters identified with the bi-directional certainty model (Bi-directional certainty map) and we performed the same additional post-hoc tests used for generating this map in order to check if the bi-directional pattern occurred also before the wagering period.

From the 47 clusters of voxels of the bi-directional certainty map, 6 clusters had bidirectional activation already at the post-match-to-sample period (see Supplementary Table 6), for example the left PMd (cluster 50-02; Fig. 15).

\section{PMd (LH) 50-02}
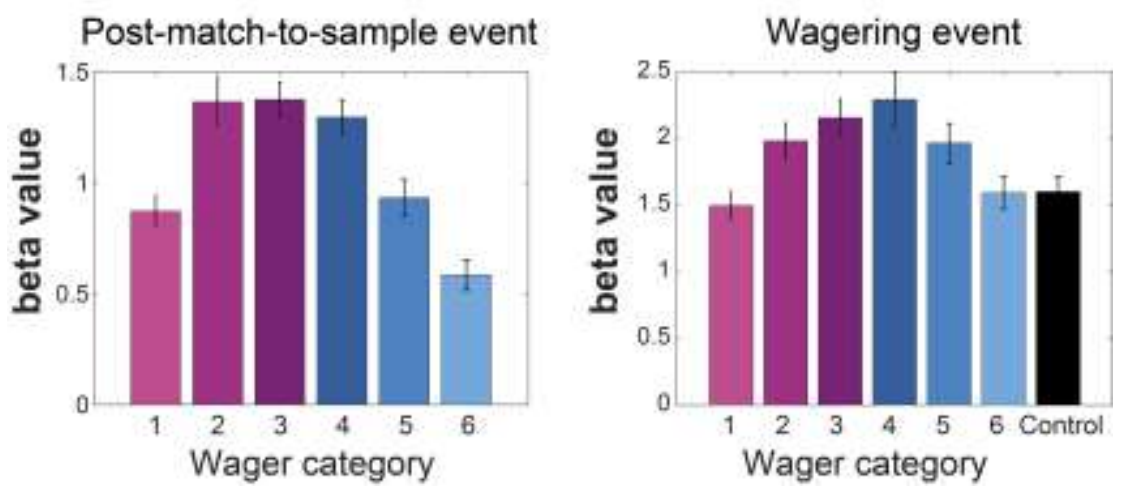

Figure 15. Beta value means and standard errors of non-parametric regressors for the post-match-tosample (left) and the wagering (right) events for the left PMd. This is one of the six areas located with the bi-directional certainty model that presented bi-directional pattern of activity already before wagering. Wagers are presented from the smallest stack (category 1, pink) to the largest stack (category 6 , light blue).

\subsection{Correlations between behavioral measures and brain activity}

Since we did not have the baseline condition necessary to directly estimate the readouts of certainty of being correct and certainty of being incorrect as done in Moreira et al. (2016), we used the differences between extremes (1 and 6) and middle (3 and 4) wagers to determine the U-shapeness, and therefore the bi-directionality, of behavioral and brain activity measures. The difference in the proportions of incorrect 
trials between wagers 1 and 3 was used as the behavioral measure for the low wagers, while the difference in the proportions of correct trials between wagers 6 and 4 was used as the behavioral measure for the high wagers. We assumed that the greater these differences (U-shape, see Figure 16A), the better subjects identified incorrect and correct perceptual decisions, respectively.

For the brain signal measures we used the wager-specific beta values calculated using the non-parametric model for the wagering event. These beta values reflect the brain activity associated to the selection of each wager. Since we only extracted beta values from clusters present in the bi-directional certainty map, we assume that they reflect wagering-related activity of areas involved in bi-directional certainty readouts. Therefore, the greater the difference between the beta values of wagers 1 and 3 (low wagers) or between the beta values of wagers 6 and 4 (high wagers) would indicate how much this cluster was modulated by bi-directional certainty readouts. We assumed that the greater these differences, the more the brain activity was modulated by certainty of being incorrect and certainty of being correct, respectively (see Figure $16 B$ for $U$-shape; and Figure 16C for inverted $U$-shape). Thus, correlations (across subjects) between the wager-specific proportions of trials and non-parametric beta values would indicate that subjects' metacognitive ability in identifying incorrect and/or correct decisions were reflected in the modulation by certainty of the activity of wagering-related areas. These correlations were performed for low and high wagers separately and together. The bi-directional measures were based on the sum of the measures for high and low wagers.

While positive values of behavioral measures are always indicative of successful behavioral performance (since they indicate that subjects better identified correct and incorrect decisions), positive or negative values of the beta values might indicate expected brain activity depending on the cluster's function of wager-specific beta values. Independently on if the beta values are below or above zero, positive differences between the wagers 1 and 3 and between the wagers 6 and 4 represent expected brain activity patterns when wager-specific beta values have a "U" shape in the group level (Fig. 16B); whereas negative differences are expected when wagerspecific beta values have an "inverted U" shape in the group level (Fig. 16C). 

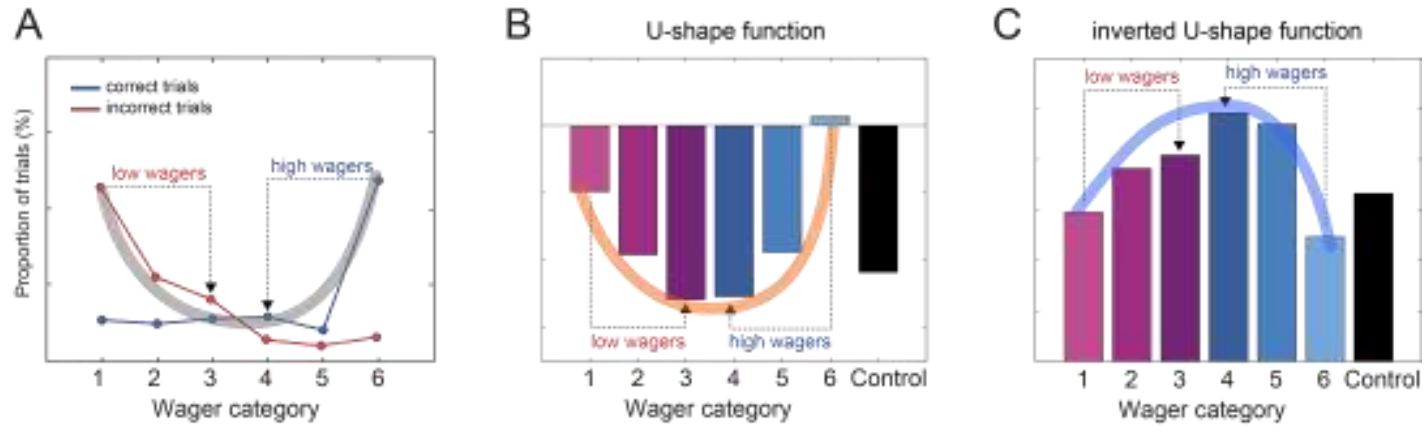

Figure 16. Behavioral and brain signal measurements used for correlations across subjects. (A) The red curve represents wager-specific proportions of incorrect trials and the blue curve represents wagerspecific proportions of correct trials. The difference in the proportions of incorrect trials between wagers 1 and 3 was used as the behavioral measure for the low wagers, while the difference in the proportions of correct trials between wagers 6 and 4 was used as the behavioral measure for the high wagers. The gray translucent line highlights the U-shape of the exemplified behavioral measure. (B and C) Beta values are presented from the smallest wager (category 1, pink) to the largest wager (category 6, light blue). As the behavioral measurements, the differences between extreme and middle wagers were the brain activity measurements used in our correlations. (B) The orange translucent line highlights the U-shape of the exemplified brain signal measure. (C) The blue translucent line highlights the inverted-U-shape of the exemplified brain signal measure. These colors match the colors present in the map of the Figure 8.

Among the 47 clusters of the bi-directional certainty map, 11 clusters presented significant correlations between bi-directional wager-specific activities and behavioral measures across subjects (Table 2). No unexpected correlation (e.g. positive correlations for clusters with inverted U-shape function of wager-specific activities) was significant. This means that, even though we modeled the brain activity using the average of wager-specific proportions of correct and incorrect trials across subjects, these behavioral measures were also associated with inter-individual differences in brain activation patterns.

\section{Table 2}

Clusters that presented significant intersubject correlation for high and low wagers

\begin{tabular}{|c|c|c|c|c|c|}
\hline Area & Hemisphere & Cluster & $\mathbf{r}$ & $\mathbf{p}$ & function \\
\hline dorsal Anterior Cingulate Cortex & Left & $46-03$ & -0.58 & 0.01 & inverted U-shape \\
\hline dorsal Premotor Cortex & Right & $46-04$ & -0.45 & 0.05 & inverted U-shape \\
\hline Precuneus & Right & $47-04$ & -0.61 & 0.005 & inverted U-shape \\
\hline
\end{tabular}




$\begin{array}{clccccc}4 & \text { Inferior Parietal Lobule } & \text { Right } & 47-05 & -0.46 & 0.04 & \text { inverted U-shape } \\ 5 & \text { Angular Gyrus } & \text { Left } & 47-08 & -0.54 & 0.01 & \text { inverted U-shape } \\ 6 & \text { Inferior Parietal Lobule } & \text { Left } & 47-09 & -0.51 & 0.02 & \text { inverted U-shape } \\ 7 & \text { Superior Occipital Lobule } & \text { Right } & 47-12 & -0.59 & 0.01 & \text { inverted U-shape } \\ 8 & \text { Superior Occipital Gyrus } & \text { Right } & 47-14 & -0.62 & 0.005 & \text { inverted U-shape } \\ 9 & \text { dorsolateral Prefrontal Cortex } & \text { Left } & 48-02 & -0.44 & 0.05 & \text { inverted U-shape } \\ 10 & \text { ventral Premotor Cortex } & \text { Left } & 49-01 & -0.47 & 0.03 & \text { inverted U-shape } \\ 11 & \text { dorsal Premotor Cortex } & \text { Left } & 50-02 & -0.47 & 0.04 & \text { inverted U-shape }\end{array}$

All the clusters that presented correlations for bi-directional measures also demonstrated correlations between behavioral and brain signal measures for high wagers. Additionally, 5 clusters presented correlations only for measures based on high wagers, adding up to 16 clusters with significant correlations (Table 3).

\section{Table 3}

Clusters that presented significant intersubject correlation for high wagers

\begin{tabular}{|c|c|c|c|c|c|c|}
\hline & Area & Hemisphere & Cluster & $\mathbf{r}$ & $\mathbf{p}$ & function \\
\hline 1 & dorsolateral Prefrontal Cortex & Right & $46-01$ & -0.45 & 0.05 & inverted U-shape \\
\hline 2 & dorsal Anterior Cingulate Cortex & Left & $46-03$ & -0.72 & 0.005 & inverted U-shape \\
\hline 3 & dorsal Premotor Cortex & Right & $46-04$ & -0.54 & 0.01 & inverted U-shape \\
\hline 4 & Inferior Parietal Lobule & Left & $47-03$ & -0.49 & 0.03 & inverted U-shape \\
\hline 5 & Precuneus & Right & $47-04$ & -0.54 & 0.01 & inverted U-shape \\
\hline 6 & Inferior Parietal Lobule & Right & $47-05$ & -0.57 & 0.01 & inverted U-shape \\
\hline 7 & Angular Gyrus & Left & $47-08$ & -0.62 & 0.005 & inverted U-shape \\
\hline 8 & Inferior Parietal Lobule & Left & 47-09 & -0.52 & 0.02 & inverted U-shape \\
\hline 9 & Inferior Parietal Lobule & Right & $47-10$ & -0.59 & 0.01 & inverted U-shape \\
\hline 10 & Superior Occipital Lobule & Right & $47-12$ & -0.76 & 0.005 & inverted U-shape \\
\hline 11 & Superior Occipital Gyrus & Right & $47-14$ & -0.60 & 0.01 & inverted U-shape \\
\hline 12 & dorsolateral Prefrontal Cortex & Left & $48-02$ & -0.67 & 0.005 & inverted U-shape \\
\hline 13 & anterior Prefrontal Cortex & Left & $48-03$ & -0.54 & 0.01 & inverted U-shape \\
\hline 14 & anterior Prefrontal Cortex & Left & $48-05$ & -0.64 & 0.005 & inverted U-shape \\
\hline 15 & ventral Premotor Cortex & Left & $49-01$ & -0.64 & 0.005 & inverted U-shape \\
\hline 16 & dorsal Premotor Cortex & Left & $50-02$ & -0.46 & 0.04 & inverted U-shape \\
\hline
\end{tabular}

Significant intersubject correlation between behavioral and brain signal measures for low wagers occurred in six clusters (Table 4). Three of them were correlations that went against the predictions (clusters: 01-25, 01-45 and 01-57, see Table 4). The cluster 5101 located at the Insula (left hemisphere) presented significant and expected correlation based only on low wagers measures. 


\section{Table 4}

Clusters that presented significant intersubject correlation for low wagers

\begin{tabular}{|c|c|c|c|c|c|c|}
\hline & Area & Hemisphere & Cluster & $\mathbf{r}$ & p & Function \\
\hline 1 & Insula & Left & $01-25$ & -0.60 & 0.01 & U-shape \\
\hline 2 & Somatosensory Assoc. Cortex & Right & $01-45$ & -0.64 & 0.01 & U-shape \\
\hline 3 & Primary Somatosensory Cortex & Right & $01-57$ & -0.61 & 0.01 & U-shape \\
\hline 4 & dorsal Anterior Cingulate Cortex & Left & $46-03$ & -0.46 & 0.04 & inverted U-shape \\
\hline 5 & Inferior Parietal Lobule & Left & 47-09 & -0.45 & 0.05 & inverted U-shape \\
\hline 6 & Insula & Left & $51-01$ & -0.49 & 0.03 & inverted U-shape \\
\hline
\end{tabular}

The dACC (Fig. 17) and the IPL were, therefore, the only areas which presented significant correlations both for high and low wagers measures (bi-directional encoding of certainty).

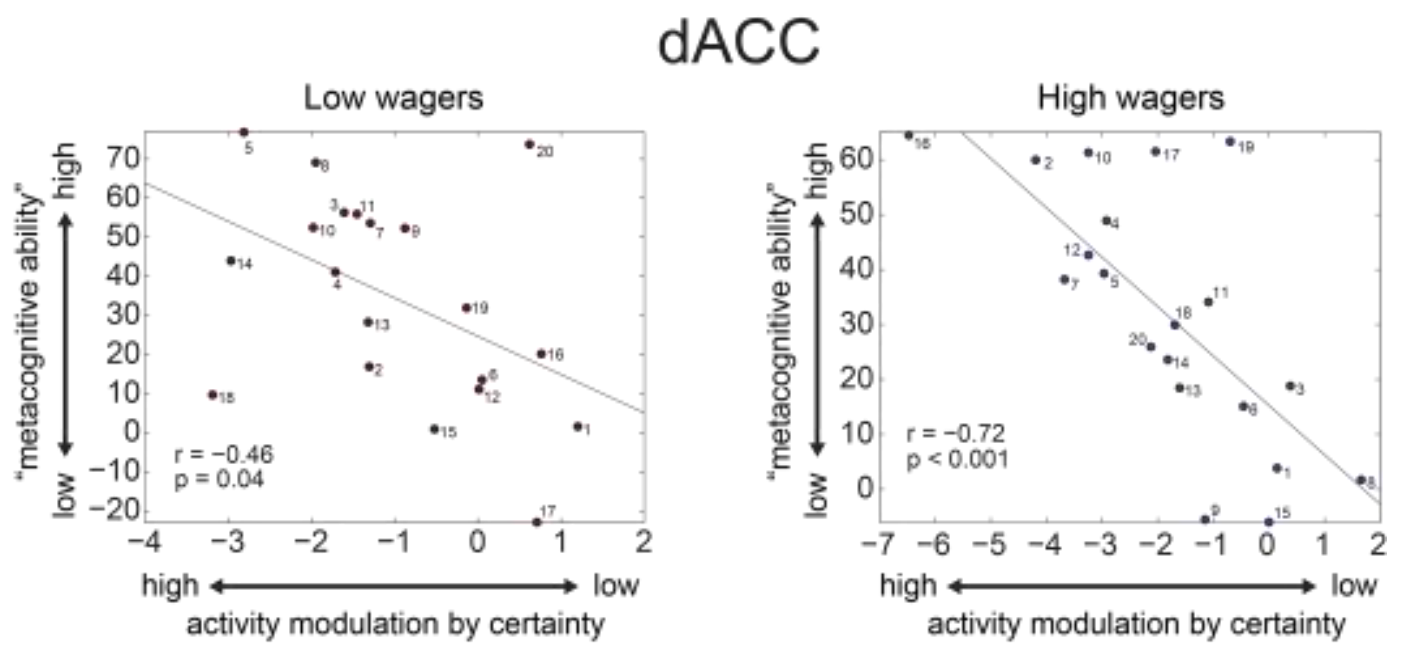

Figure 17. Correlations between behavioral and brain signal measures for the dorsal anterior cingulate cortex (dACC). On the left, the correlation across subjects for the difference between the proportions of incorrect trials (y-axis) and the beta values (x-axis) of wagers 1 and 3. On the right, the correlation for the difference between the proportions of correct trials and the beta values of wagers 6 and 4 . These correlations indicate that subjects who better identified correct and incorrect trials had the activity of dACC more modulated by certainty of being correct and certainty of being incorrect, respectively. Similar pattern was found for the inferior parietal lobule (IPL, not shown).

We also performed correlations between wager-specific beta value and wager-specific performance, separately for each wager. There were no significant correlations. This result indicates that wager-specific proportions of correct and incorrect trials can 
predict better the brain activity patterns than the performance associated to each wager, providing support for the use of the wager-specific proportions of trials as the reference behavior for our fMRI models.

Contrary to Fleming et al. (2012), we did not find correlations between the estimates of the bi-directional certainty parametric regressor and metacognitive sensitivity, except a correlation for one cluster in the left aPFC $(r=-0.54, p<0.05)$ that indicates higher metacognitive sensitivity for subjects with beta estimate of wager-related activity value closer to zero (the opposite of the expected pattern).

\section{Discussion}

Certainty readouts are ubiquitous processes intimately related to routine decisionmaking. In the present study, we showed that several brain areas are able to encode these readouts in a bi-directional way (i.e. certainty of being correct and certainty of being incorrect), suggesting a distributed processing based on certainty-related information. Furthermore, we demonstrated that these areas were not involved in encoding the difficulty of perceptual decisions, but some of them were involved in the evaluation of post-decisional information that might have resulted in certainty-related bi-directional reports. These areas were mostly located in the frontal, parietal and cingulate cortices, and several of them were previously related to readouts of certainty of being correct (e.g. aPFC in Fleming et al., 2012; IPS as the human homologous for the monkey intraparietal lateral area in Kiani and Shadlen, 2009; dACC in Hebart et al., 2014; dlPFC in Heereman et al., 2015) or certainty of being incorrect (e.g. dACC in Charles et al., 2013; PCC in Agam et al., 2011).

Most of the parietal and frontal areas previously related to unidirectional readouts of certainty of being correct were only present in the bi-directional map, suggesting that our task requirements prompted these areas to encode the information in the most adaptive way (i.e. profit more when correct and avoid large losses when incorrect). We suggest that, since adaptive wagering reflects the proper assessment of the wager options utility (reflecting certainty only implicitly), the information that led to such flexible behavior should be based on the reliability of the sensory evidence (i.e. certainty). 
We searched for neural representations of certainty using models based on the behavioral data. According to this data, subjects were using the six-grade wager scale exponentially in order to maximize gains and minimize losses, meaning that the frequency that subjects were assigning wagers to correct trials increased exponentially towards the highest wager; whereas the frequency that subjects were assigning wagers to incorrect trials increased exponentially towards the lowest wager. Since it is assumed that subjects report their certainty implicitly during PDW (Pouget et al., 2016), we interpret our results as a demonstration that increments of evidence increased subjects' certainty exponentially. Previous studies, on the other hand, assumed that cognitive processes transform the decision variable (DV) into certainty linearly (e.g. Fleming et al., 2012; Hebart et al., 2014; Heereman et al., 2015). To test this assumption, we compared a linear model to the (exponential) certainty of being correct model, both unidirectional. According to our results, the model based on an exponential function explains the brain activity more efficiently than the linear model. The activity of more than two thirds of the areas located with the linear model presented exponential relationship determined by the additional post-hoc tests, while only $16 \%$ of those areas presented linear relationship, even under more permissive threshold. These results provided further support for the use of wager-specific proportions of correct and incorrect trials as the reference behavioral measure for the modulation of certainty-related parametric regressors.

Additionally, we found correlations between wager-specific proportions of correct and incorrect trials and wager-specific beta values, across subjects. These results suggest that the right branch of the bi-directional certainty-related signal we recorded during the period following wagering was associated with the assignment of wagers to correct trials, whereas the left branch was associated to PDW after incorrect trials. The association for high wagers was more robust since sixteen areas presented correlation between the brain activity and behavior for those wagers. The number of areas decreased to three for the expected correlations regarding the low wagers. These results indicate that, since low wagers were significantly less often used than the highest wager (see Supplementary Fig. 12), the strength of the correlation between brain signal and behavior might also depend on the general frequency each wager was utilized. But it also might indicate that, more than the real wager-specific proportions of correct and incorrect trials, subjects' belief about the correctness of their decisions 
influenced certainty-related representations in the brain (Ma and Jazayeri, 2014). Nevertheless, two areas presented consistent bi-directional correlations between brain signal and behavior: the dorsal anterior cingulate cortex (dACC) and the inferior parietal lobule (IPL). dACC was separately associated with both certainty directions in several studies (certainty of being correct: Baird et al., 2013; Hebart et al., 2014; Heereman et al., 2015; Fleming et al., 2012; and certainty of being incorrect: Agam et al., 2011; Charles et al., 2013; Hochman et al., 2014) probably because of its general role in conflict monitoring and behavioral adjustment (Kerns et al., 2004), which are important cognitive functions to generate adaptive behavior based on certainty readouts, independently on the readout direction. The IPL, together with the intraparietal sulcus (IPS), is involved in motor planning modulated by evidence strength (e.g. in monkeys: Kiani and Shadlen, 2009; in humans: Filimon et al., 2013) and, conversely, task-independent certainty assessments (Heereman et al., 2015). Since we found - as in Moreira et al., 2016 - bi-directionality in wager-specific RT2 (faster reaction times associated with the lowest and the highest wagers compared to the middle ones), the IPL could be involved in the motor preparation that led to fast responses modulated by high levels of certainty of being correct or certainty of being incorrect. Nevertheless, both IPL and IPS presented bi-directional readouts during the rest position period 3 (before wagering), revealing that bi-directional brain signal cannot be explained only as consequence of RT2 (unless non-specific motor preparation takes place during this phase). In fact, despite the bi-directional pattern of wager-specific RT2, primary motor areas and regions of the cerebellum were not present in the bi-directional certainty map, but in the certainty of being correct map, suggesting that bi-directional processes were not directly associated with motor responses. We did not find correlations between subjects' metacognitive sensitivity (meta-d') and brain activity, even for the anterior prefrontal cortex (aPFC) which showed such correlation in Fleming et al., (2012; see Supplementary Fig. 13).

The association between belief, certainty and utility is very important for the understanding of reward-based decisions as PDW (De Martino et al., 2012). In the present experiment, areas located with the parametric regressors for difficulty levels (match-to-sample event) and the parametric regressor for certainty of being correct (wagering event) can be both related to reward expectation since subjects wagered higher after easier trials, probably expecting to profit more. Moreover, certainty can 
be directly influenced by the difficulty of perceptual decisions as predicted by the attractor model of Insabato et al. (2010) and demonstrated by studies with different animals, including humans (Kepecs et al., 2008; Kiani and Shadlen, 2009; Rolls et al., 2010). The comparisons between the match-to-sample and the wagering events revealed that two clusters, one situated at the left thalamus and the other one at the right dorsal premotor cortex (PMd), were associated with representations of different difficulty levels and certainty of being correct. The thalamus is heavily interconnected with the prefrontal cortex and receives inputs from the amygdala and ventral striatum. This subcortical brain area was associated with reward-guided behavioral updates in environments with high reward stochasticity (Chakraborty et al., 2016). Moreover, the neural activity of one of its nuclei, the pulvinar, was directly implicated in rewardbased confidence reports during visual categorization in monkeys (Komura et al., 2013). Regarding the PMd, Fleming et al. (2015) selectively disrupted post-decision confidence in visual discrimination judgments by applying TMS to subjects' PMd. Although confidence about perceptual decisions is commonly associated with the strength of the supporting evidence (for review see Yeung \& Summerfield, 2012), motor-related circuits have been implicated in encoding perceptual decision (Cisek \& Kalaska, 2005; Shadlen \& Newsome, 2001), suggesting an "embodied" system that also contributes to visual confidence (Filimon et al., 2013). Also considering the connection between PMd and areas from the lateral prefrontal cortex associated with metacognition (Fleming et al., 2012; Rounis et al., 2010), our results provide extra evidence for the association between this motor-related area with certainty-related processing, supporting the existence of an "embodied" mechanism that contributes to metacognition.

Since the ventral striatum is involved in reward prediction error (e.g. O'Doherty et al. 2004), motivation in general (Talmi et al. 2008), as well as in certainty representations (Hebart et al., 2014), we extracted this area's beta values using the coordinates reported by Hebart et al. (2014) and, afterwards, we performed the additional post-hoc tests related to difficulty levels and certainty of being correct. The right and the left ventral striatum showed clear patterns predicted by both tests (see Supplementary Fig. 10). The task used by Hebart et al. (2014) contained only one difficulty level. In this context, the signal in the ventral striatum was assumed to reflect subjects' confidence in association to readouts of the activity in the right 
ventrolateral prefrontal cortex. Although we do not consider the reports using explicit confidence scale as reward-based decisions, the feeling of being confident was also associated with reward (Hebart et al., 2014). Together with our findings, and considering that PDW is a reward-based decision, we propose that the areas which presented BOLD signal patterns predicted for representation of the trial difficulty level and certainty of being correct readouts (ventral striatum, PMd and thalamus) were also associated with reward prediction.

Finally, most subjects of the present experiment (18 from 20) presented high metacognitive efficiency (meta-d'>d'), suggesting that they improved their wagering performance by using post-decisional information (Moreira et al., 2016). Postdecisional information contains higher-order representations of error likelihood in addition to the accumulated perceptual evidence (Murphy et al., 2015). Here, we assumed that the left branch of bi-directional activations also represented both error likelihood and the reliability of the inconsistent perceptual evidence (i.e. evidence whose post-decisional drift directed to the non-selected decision option). It is important to emphasize, however, that these representations are not completely overlapping. While the perceptual evidence should correspond to a closer representation of the physical visual stimulation in addition to the inherent stochasticity of the nervous system during this perception evidence processing, the error likelihood (although based on the perceptual evidence) represents more subjective distributions. In this context, the left branch of bi-directional activations is not only associated with incorrect trials, but also with correct trials perceived as probably incorrect by the subjects. Nevertheless, since certainty of being incorrect increased on easier trials (which was indicated by the pre-experiment and by the increased difference between wager-specific proportions of correct and incorrect trials following easier decisions inside the scanner; see Supplementary Fig. 14), error likelihood should not be mistaken for low levels of certainty.

\section{Summary}

Flexible PDW (gain more when correct and avoid large losses when incorrect) prompted several brain areas to encode certainty in a bi-directional manner. Fewer areas encoded unidirectional certainty readouts. Among them PMd, thalamus and 
ventral striatum represented difficulty levels and certainty of being correct-related signals, and were associated with reward prediction. Furthermore, we demonstrated that areas involved in bi-directional certainty readouts were involved in the evaluation of post-decisional information prior to the wagering event, which might have resulted in the certainty-related bi-directional reports. Finally, report-related behavioral patterns (specifically the inverted U-shape function of wager-specific RT2) did not explain by themselves the activity patterns of motor-related areas; instead, our fMRI and behavioral results support the existence of a distributed system that contributes to the evaluation of perceptual certainty. 


\section{Supplementary information}

Regressors timing. The onsets and duration of the main regressors are described in the Supplementary Table 1.

\section{Supplementary Table 1}

Onset and duration of the main regressors

\begin{tabular}{|l|l|l|}
\hline Regressor & Onset & Duration \\
\hline Fixation & beginning of the rest position period 1 & $0.2 \mathrm{~s}$ \\
Sample & sample presentation & $1 \mathrm{~s}$ \\
Match-to-sample & match-to-sample presentation & mean RT1 \\
Post-match-to-sample & $1.5 \mathrm{~s}$ after match-to-sample presentation & mean RT1 \\
Wagering & Presentation of the wagering task & mean RT2 \\
Control & presentation of the control task & mean RT2 \\
\hline
\end{tabular}

Cluster tables. The relationship between the different modeled events and brain activity was analyzed for each subject separately. Subject-estimates were tested against zero (t-test) to assess group level significance. Subsequently, significant brain activations for those t-tests were FDR corrected (FDR <0.05) and had to fit the additional post-hoc tests criteria to be included in the maps presented in the Results section and in their respective cluster tables (see below).

Since, in some cases, different clusters were located in the same brain area, we identified the clusters with numbers (last column of the cluster tables) in order to cross the information presented in the figures of the Results section and the following Supplementary Tables. The clusters were extracted using NeuroElf (www.neuroelf.net) and the brain areas identified according to the atlas of the Talairach Project at the Research Imaging Institute of the University of Texas Health Science Center San Antonio (http://www.talairach.org/applet.html).

37 clusters presented activity varying parametrically according to the five difficulty levels of the perceptual decision (match-to-sample event; Supplementary Table 2). 


\section{Supplementary Table 2}

Clusters which encoded difficulty levels (match-to-sample event)

\begin{tabular}{|c|c|}
\hline & Name \\
\hline 1 & dorsal Premotor Cortex \\
\hline 2 & dorsal Premotor Cortex \\
\hline 3 & Middle Frontal Gyrus \\
\hline 4 & dorsal Anterior Cingulate Cortex \\
\hline 5 & dorsal Anterior Cingulate Cortex \\
\hline 6 & dorsal Anterior Cingulate Cortex \\
\hline 7 & dorsal Anterior Cingulate Cortex \\
\hline 8 & dorsolateral Prefrontal Cortex \\
\hline 9 & Inferior Parietal Lobule \\
\hline 10 & dorsal Premotor Cortex \\
\hline 11 & Supplementary Motor Area \\
\hline 12 & dorsolateral Prefrontal Cortex \\
\hline 13 & Lentiform Nucleus \\
\hline 14 & Lentiform Nucleus \\
\hline 15 & Lentiform Nucleus \\
\hline 16 & Thalamus \\
\hline 17 & Lentiform Nucleus \\
\hline 18 & Middle Temporal Gyrus \\
\hline 19 & Middle Occipital Gyrus \\
\hline 20 & Middle Occipital Gyrus \\
\hline 21 & Fusiform Gyrus \\
\hline 22 & Middle Occipital Gyrus \\
\hline 23 & dorsal Posterior Cingulate \\
\hline 24 & Precuneus \\
\hline 25 & Precuneus \\
\hline 26 & dorsal Posterior Cingulate Cortex \\
\hline 27 & dorsal Posterior Cingulate Cortex \\
\hline 28 & Lingual Gyrus \\
\hline 29 & Middle Occipital Gyrus \\
\hline 30 & Declive \\
\hline 31 & Parahippocampal Gyrus \\
\hline 32 & Precuneus \\
\hline 33 & Precuneus \\
\hline 34 & Fusiform Gyrus \\
\hline 35 & Medial Frontal Gyrus \\
\hline 36 & Middle Temporal Gyrus \\
\hline 37 & Middle Temporal Gyrus \\
\hline
\end{tabular}

\begin{tabular}{|c|c|c|c|c|c|}
\hline Hemisphere & $\mathrm{x}$ & $\mathrm{y}$ & $\mathrm{z}$ & $\mathrm{t}$ value & Cluster \\
\hline Right & 12 & 14 & 61 & 5.74 & 01-01 \\
\hline Right & 12 & 23 & 55 & 4.18 & 01-02 \\
\hline Right & 33 & 23 & 40 & 5.57 & 02-01 \\
\hline Right & 12 & 17 & 31 & 5.3 & 03-01 \\
\hline Right & 3 & 20 & 40 & 4.74 & 03-02 \\
\hline Left & -6 & 26 & 34 & 4.65 & 03-03 \\
\hline Left & -6 & 17 & 40 & 4.1 & 03-04 \\
\hline Right & 15 & 38 & 37 & 5.28 & 04-01 \\
\hline Right & 45 & -43 & 34 & 5.05 & $05-01$ \\
\hline Left & -12 & 8 & 61 & 5.04 & 06-01 \\
\hline Left & -12 & 8 & 52 & 4.65 & $06-02$ \\
\hline Right & 39 & 41 & 22 & 4.23 & $10-01$ \\
\hline Right & 15 & 5 & -5 & -9.36 & $12-01$ \\
\hline Left & -15 & 5 & -2 & -7.1 & $12-02$ \\
\hline Left & -27 & -7 & -8 & -6.96 & $12-03$ \\
\hline Left & 0 & -7 & 10 & -5.12 & $12-04$ \\
\hline Left & -24 & -1 & 10 & -4.97 & $12-05$ \\
\hline Right & 39 & -76 & 10 & -6.19 & $13-01$ \\
\hline Right & 27 & -88 & 13 & -5.76 & $13-02$ \\
\hline Right & 36 & -85 & 1 & -4.47 & $13-04$ \\
\hline Right & 39 & -70 & -11 & -4.44 & $13-05$ \\
\hline Right & 36 & -82 & -8 & -4.09 & $13-06$ \\
\hline Right & 15 & -37 & 31 & -5.85 & $14-01$ \\
\hline Left & 0 & -52 & 37 & -5.61 & $15-01$ \\
\hline Left & -24 & -55 & 55 & -5.32 & $16-01$ \\
\hline Left & -3 & -31 & 34 & -5.26 & $17-01$ \\
\hline Left & -15 & -25 & 37 & -4.5 & $17-02$ \\
\hline Right & 3 & -88 & -5 & -4.93 & $19-02$ \\
\hline Left & -33 & -82 & -8 & -4.98 & $20-01$ \\
\hline Left & 0 & -58 & -14 & -4.18 & $22-02$ \\
\hline Right & 42 & -43 & -5 & -4.76 & 23-01 \\
\hline Left & -3 & -61 & 19 & -4.7 & 24-01 \\
\hline Left & -12 & -58 & 19 & -4.37 & 24-02 \\
\hline Left & -45 & -70 & -11 & -4.52 & $26-01$ \\
\hline Left & -6 & 50 & 7 & -4.52 & $27-01$ \\
\hline Left & -42 & -67 & 28 & -4.38 & $31-01$ \\
\hline Left & -42 & -73 & 19 & -3.91 & $31-02$ \\
\hline
\end{tabular}

As one of our main results, 47 clusters encoded certainty in a bi-directional way (wagering event; Supplementary Table 3). Several areas were located at the frontal, parietal and cingulate cortices. 


\section{Supplementary Table 3}

Clusters which encoded both certainty directions (wagering event)

\begin{tabular}{|c|c|}
\hline \\
\hline \multicolumn{2}{|c|}{$\begin{aligned} & \text { Name } \\
1 & \text { rostral }\end{aligned}$} \\
\hline & rentral Posterior Cingulate Cortex \\
\hline 3 & Precuneus \\
\hline 4 & Superior Temporal Gyrus \\
\hline 5 & Superior Temporal Gyrus \\
\hline 6 & Middle Temporal Gyrus \\
\hline 7 & Caudate \\
\hline 8 & Insula \\
\hline 9 & Anterior Cingulate \\
\hline 10 & Insula \\
\hline 11 & rostral Anterior Cingulate Cortex \\
\hline 12 & Superior Temporal Gyrus \\
\hline 13 & Middle Temporal Gyrus \\
\hline 14 & Superior Frontal Gyrus \\
\hline 15 & anterior Prefrontal Cortex \\
\hline 16 & Somatosensory Association Cortex \\
\hline 17 & Cingulate Gyrus \\
\hline 18 & Insula \\
\hline 19 & Primary Somatosensory Cortex \\
\hline 20 & Inferior Frontal Gyrus \\
\hline 21 & Superior Frontal Gyrus \\
\hline 22 & dorsolateral Prefrontal Cortex \\
\hline 23 & dorsal Anterior Cingulate Cortex \\
\hline 24 & dorsal Premotor Cortex \\
\hline 25 & dorsal Premotor Cortex \\
\hline 26 & (Pre) Supplementary Motor Area \\
\hline 27 & Precuneus \\
\hline 28 & Inferior Parietal Lobule \\
\hline 29 & Inferior Parietal Lobule \\
\hline 30 & Precuneus \\
\hline 31 & Inferior Parietal Lobule \\
\hline 32 & Inferior Parietal Lobule \\
\hline 33 & Intraparietal Sulcus \\
\hline 34 & Angular Gyrus \\
\hline 35 & Inferior Parietal Lobule \\
\hline 36 & Inferior Parietal Lobule \\
\hline 37 & Intraparietal Sulcus \\
\hline 38 & Superior Occipital Lobule \\
\hline 39 & Superior Occipital Gyrus \\
\hline 40 & dorsolateral Prefrontal Cortex \\
\hline & anterior Prefrontal Cort \\
\hline
\end{tabular}

\begin{tabular}{|c|c|c|c|c|c|}
\hline Hemisphere & $\mathbf{x}$ & $\mathbf{y}$ & $\mathbf{z}$ & t value & Cluster \\
\hline Left & -9 & 44 & -2 & 9.28 & 01-01 \\
\hline Left & -9 & -52 & 16 & 8.6 & 01-02 \\
\hline Left & -12 & -46 & 28 & 7.58 & 01-03 \\
\hline Right & 51 & 17 & -17 & 6.26 & $01-10$ \\
\hline Left & -54 & 11 & -14 & 6.09 & $01-11$ \\
\hline Left & -57 & -4 & -11 & 5.93 & $01-17$ \\
\hline Left & -21 & 17 & 22 & 5.88 & 01-19 \\
\hline Right & 30 & -28 & 25 & 5.48 & $01-23$ \\
\hline Right & 18 & 26 & 16 & 5.52 & $01-24$ \\
\hline Left & -33 & -22 & 25 & 5.39 & $01-25$ \\
\hline Right & 15 & 41 & 7 & 5.39 & $01-26$ \\
\hline Right & 51 & 8 & -14 & 5.33 & $01-27$ \\
\hline Right & 54 & -16 & -5 & 5.06 & $01-30$ \\
\hline Left & -15 & 41 & 52 & 4.82 & $01-37$ \\
\hline Left & -12 & 62 & 10 & 4.79 & $01-44$ \\
\hline Right & 30 & -40 & 64 & 5.16 & $01-45$ \\
\hline Right & 18 & 14 & 25 & 4.51 & $01-51$ \\
\hline Left & -27 & -34 & 25 & 4.47 & $01-52$ \\
\hline Right & 24 & -31 & 67 & 4.43 & $01-57$ \\
\hline Right & 33 & 26 & -11 & 3.81 & $01-87$ \\
\hline Left & -6 & 59 & 40 & 3.71 & 01-89 \\
\hline Right & 42 & 23 & 34 & -7.75 & $46-01$ \\
\hline Left & 0 & 20 & 43 & -6.21 & $46-03$ \\
\hline Right & 21 & 2 & 55 & -5.38 & $46-04$ \\
\hline Right & 30 & 11 & 61 & -4.69 & $46-07$ \\
\hline Left & -9 & 8 & 49 & -3.89 & $46-12$ \\
\hline Right & 6 & -70 & 43 & -6.5 & $47-01$ \\
\hline Left & -51 & -46 & 40 & -6.27 & $47-02$ \\
\hline Left & -42 & -46 & 37 & -5.92 & $47-03$ \\
\hline Right & 15 & -67 & 40 & -5.85 & $47-04$ \\
\hline Right & 39 & -43 & 43 & -5.74 & $47-05$ \\
\hline Left & -45 & -55 & 40 & -5.68 & $47-06$ \\
\hline Left & -18 & -70 & 40 & -5.31 & $47-07$ \\
\hline Left & -33 & -61 & 37 & -5.2 & $47-08$ \\
\hline Left & -33 & -46 & 37 & -4.98 & 47-09 \\
\hline Right & 48 & -37 & 43 & -4.91 & $47-10$ \\
\hline Right & 24 & -70 & 34 & -4.76 & $47-11$ \\
\hline Right & 30 & -61 & 34 & -4.75 & $47-12$ \\
\hline Right & 36 & -73 & 25 & -4.17 & $47-14$ \\
\hline Left & -39 & 26 & 34 & -5.83 & $48-02$ \\
\hline Left & -45 & 44 & 10 & -5.24 & $48-03$ \\
\hline
\end{tabular}




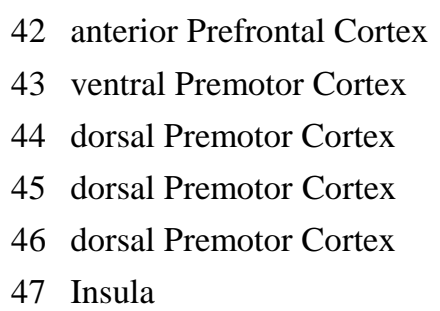

$\begin{array}{lccccc}\text { Left } & -30 & 47 & 19 & -4.58 & 48-05 \\ \text { Left } & -42 & -1 & 37 & -5.44 & 49-01 \\ \text { Left } & -24 & -1 & 61 & -5.46 & 50-01 \\ \text { Left } & -21 & -1 & 52 & -4.84 & 50-02 \\ \text { Left } & -24 & 8 & 52 & -4.67 & 50-03 \\ \text { Left } & -30 & 14 & 10 & -5.03 & 51-01\end{array}$

41 clusters encoded certainty of being correct (wagering event; Supplementary Table 4). Several clusters were not represented or incompletely represented on the inflated brain, such as: Caudate, Declive (cerebellum), Pulvinar, Insula and Parahippocampal Gyrus.

\section{Supplementary Table 4}

Clusters which encoded certainty of being correct (wagering event)

\begin{tabular}{|c|c|c|c|c|c|c|c|}
\hline & Name & Hemisphere & $\mathbf{x}$ & $\mathbf{y}$ & $\mathbf{z}$ & t value & Cluster \\
\hline 1 & Declive (Cerebellum) & Left & 0 & -70 & -14 & 8.17 & $01-01$ \\
\hline 2 & Thalamus (Pulvinar) & Left & -24 & -25 & 13 & 7.54 & 01-02 \\
\hline 3 & rostral Anterior Cingulate Cortex & Left & -15 & 35 & 4 & 7.21 & $01-03$ \\
\hline 4 & Caudate & Left & -12 & 26 & 4 & 6.77 & 01-04 \\
\hline 5 & Thalamus & Left & 0 & -10 & 10 & 6.2 & $01-05$ \\
\hline 6 & Anterior Cingulate & Right & 15 & 32 & 7 & 6.18 & $01-06$ \\
\hline 7 & Caudate & Left & -30 & -25 & -2 & 6.07 & 01-08 \\
\hline 8 & ventral Posterior Cingulate Cortex & Left & -12 & -55 & 22 & 5.87 & $01-09$ \\
\hline 9 & ventral Posterior Cingulate Cortex & Left & -9 & -52 & 13 & 5.63 & $01-10$ \\
\hline 10 & Insula & Left & -24 & 11 & 19 & 5.61 & $01-11$ \\
\hline 11 & Superior Temporal Gyrus & Left & -36 & -37 & 13 & 5.59 & $01-12$ \\
\hline 12 & Sub-Gyral & Right & 30 & -34 & -2 & 5.52 & $01-13$ \\
\hline 13 & Caudate & Left & -15 & -13 & 22 & 5.64 & $01-14$ \\
\hline 14 & Caudate & Left & -21 & -4 & 25 & 5.51 & $01-15$ \\
\hline 15 & Primary Motor Cortex & Right & 45 & -10 & 52 & 5.63 & $01-17$ \\
\hline 16 & ventral Anterior Cingulate Cortex & Right & 3 & 29 & 10 & 5.38 & $01-18$ \\
\hline 17 & Declive (Cerebellum) & Right & 18 & -67 & -20 & 5.31 & $01-20$ \\
\hline 18 & Lentiform Nucleus & Left & -18 & -7 & -2 & 5.2 & $01-21$ \\
\hline 19 & Posterior Occipital Lobule & Right & 18 & -76 & 7 & 5.23 & $01-22$ \\
\hline 20 & Anterior Cingulate & Right & 24 & 35 & 7 & 5.16 & $01-23$ \\
\hline 21 & Posterior Cingulate & Right & 30 & -70 & 7 & 5.17 & $01-24$ \\
\hline 22 & Claustrum & Right & 24 & 8 & 19 & 5.08 & $01-25$ \\
\hline 23 & Superior Temporal Gyrus & Left & -36 & -49 & 13 & 4.95 & $01-27$ \\
\hline 24 & Superior Temporal Gyrus & Left & -51 & 8 & -14 & 4.89 & $01-29$ \\
\hline 25 & Posterior Occipital Lobule & Left & -15 & -88 & 7 & 4.83 & $01-31$ \\
\hline 26 & Caudate & Right & 21 & 20 & 16 & 4.85 & $01-32$ \\
\hline 27 & Parahippocampal Gyrus & Left & -36 & -43 & 1 & 4.71 & $01-35$ \\
\hline 28 & Parahippocampal Gyrus & Right & 30 & -55 & 4 & 4.67 & $01-37$ \\
\hline
\end{tabular}




\begin{tabular}{|c|c|c|c|c|c|c|c|}
\hline 29 & rostral Anterior Cingulate & Left & -15 & 26 & 16 & 4.64 & $01-38$ \\
\hline 30 & Posterior Occipital Lobule & Left & -30 & -82 & 1 & 4.6 & $01-39$ \\
\hline 31 & Superior Temporal Gyrus & Right & 36 & -46 & 7 & 4.51 & $01-40$ \\
\hline 32 & Caudate & Right & 21 & -13 & 28 & 4.47 & $01-43$ \\
\hline 33 & Posterior Occipital Lobule & Right & 33 & -79 & 1 & 4.44 & $01-45$ \\
\hline 34 & Thalamus (Pulvinar) & Right & 21 & -28 & 10 & 4.26 & $01-47$ \\
\hline 35 & Precuneus & Right & 9 & -49 & 49 & 4.17 & $01-49$ \\
\hline 36 & Caudate & Right & 12 & 17 & 10 & 4.17 & $01-52$ \\
\hline 37 & Precuneus & Right & -12 & -49 & 37 & 4.22 & $01-56$ \\
\hline 38 & Posterior Occipital Lobule & Left & -15 & -82 & 25 & 3.71 & $01-76$ \\
\hline 39 & Superior Temporal Gyrus & Left & -48 & -16 & -2 & 3.69 & $01-78$ \\
\hline 40 & Supplementary Motor Area & Left & 0 & -10 & 58 & 4.58 & $04-01$ \\
\hline 41 & dorsal Premotor Cortex & Right & 15 & 14 & 61 & -4.95 & $28-01$ \\
\hline
\end{tabular}

Only seven areas presented activity varying parametrically according to certainty of being incorrect (wagering event; Supplementary Table 5), all of them located at the posterior part of the brain.

\section{Supplementary Table 5}

Clusters which encoded certainty of being incorrect (wagering event)

$\begin{array}{ll} & \text { Name } \\ 1 & \text { Middle Temporal Gyrus } \\ 2 & \text { Middle Occipital Gyrus } \\ 3 & \text { Middle Occipital Gyrus } \\ 4 & \text { Cuneus } \\ 5 & \text { Cuneus } \\ 6 & \text { Parahippocampal Gyrus } \\ 7 & \text { Fusiform Gyrus }\end{array}$

$\begin{array}{cccccc}\text { Hemisphere } & \mathbf{x} & \mathbf{y} & \mathbf{z} & \mathbf{t} \text { value } & \text { Cluster } \\ \text { Left } & -33 & -73 & 13 & -5.47 & 06-01 \\ \text { Left } & -36 & -73 & 4 & -5.27 & 06-02 \\ \text { Left } & -30 & -82 & 4 & -5.14 & 06-03 \\ \text { Left } & -15 & -79 & 7 & -4.84 & 06-04 \\ \text { Right } & 21 & -76 & 7 & -4.64 & 10-02 \\ \text { Left } & -36 & -43 & -5 & -5.21 & 12-01 \\ \text { Left } & -33 & -52 & -17 & -5.45 & 12-02\end{array}$

Lastly, from the 47 clusters from the bi-directional certainty map, 6 were present also in the post-match-to-sample map (Supplementary Table 6).

\section{Supplementary Table 6}

Clusters which encoded both certainty directions before (post-match-to-sample event) and during wagering (wagering event)

\footnotetext{
Name

1 Precuneus

2 Angular Gyrus

3 Inferior Parietal Lobule

4 Intraparietal Sulcus

5 anterior Prefrontal Cortex

6 ventral Premotor Cortex
}

$\begin{array}{cccccc}\text { Hemisphere } & \mathbf{x} & \mathbf{y} & \mathbf{z} & \mathbf{t} \text { value } & \text { Cluster } \\ \text { Right } & 15 & -67 & 40 & -5.85 & 47-04 \\ \text { Left } & -33 & -61 & 37 & -5.2 & 47-08 \\ \text { Left } & -33 & -46 & 37 & -4.98 & 47-09 \\ \text { Right } & 24 & -70 & 34 & -4.76 & 47-11 \\ \text { Left } & -45 & 44 & 10 & -5.24 & 48-03 \\ \text { Left } & -42 & -1 & 37 & -5.44 & 49-01\end{array}$


Performance per difficulty level. As described in the section 3.1 Behavioral results, performance decreased with increasing difficulty level. Performance per difficulty level was best described by a quadratic function (Supplementary Fig. 1) compared to linear and exponential functions. AIC values for linear, quadratic and exponential fits were $-503,-513$ and -495 , respectively.

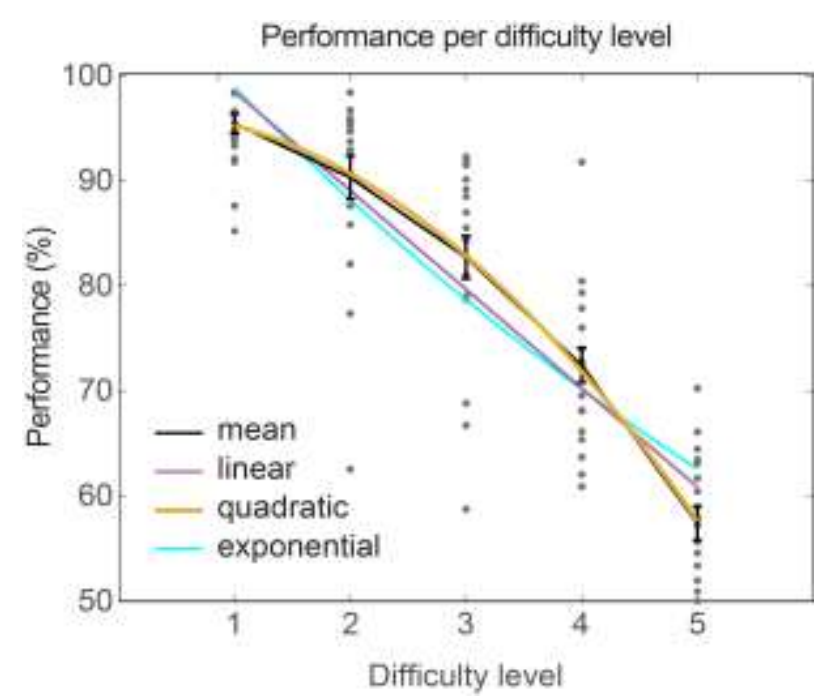

Supplementary Figure 1. Fit curves (linear, quadratic and exponential), subjects' means (dots) and group means and standard errors (black curve) of performance per difficulty level.

Parametric weights calculations. Each best fitted function provided the parametric weights associated to each difficulty level or each wager category. Specifically, the parametric weights for match-to-sample regressor were calculated by including the difficulty levels (diff: 1 to 5 ) in the fitted quadratic function and getting the corresponding weight value ( weight $_{\text {diff }}=a^{*} \operatorname{diff}+b^{*} \operatorname{diff}^{2}$ ). The parametric weights for the certainty of being correct and certainty of being incorrect regressors were calculated by including the wager categories (wager: 1 to 6 ) in the respective fitting function $\left(\right.$ weight $_{\text {wager }}=a^{*} \exp \left[b^{*}\right.$ wager $]$ ). For the right branch of the bi-directional modulator, we used the unidirectional exponential function for correct trials. In order to preserve the exponential relationship between the weights, we used the values 1 , 3.5 and 6 to calculate weights for the wagers 4,5 and 6 . The left branch was calculated with the unidirectional exponential function for incorrect trials, using the values $6,3.5$ and 1 to calculate weights for the wagers 1,2 and 3. All parametric 
weights were normalized by the minimum weight value calculated by the corresponding function.

It was essential to have unidirectional regressors (i.e. increase of values only in one direction) for the certainty of being correct and certainty of being incorrect models because certainty bi-directionality was tested in the third model. Considering that we obtained bi-directional curves by fitting wager-specific proportions of trials using three parameters, we set the maximum number of fitting function parameters as two.

Fit curves on subjects' wagering criteria. Most studies about certainty of being correct used linear parametric regressors in their certainty-related models to locate brain areas encoding confidence (e.g. Hebart et al., 2014). We, instead, have two behavioral indications that subjects were using wagers non-linearly to report their certainties: wagers proportions of correct and incorrect trials and subjects' criteria to select the wagers, both better described by exponential functions compared to linear and quadratic ones (Supplementary Fig. 2). Subjects' wagering criteria were calculated using Maniscalco and Lau's code provided at: http://www.columbia.edu/ bsm2105/type2sdt/archive/index.html. AIC values for linear, quadratic and exponential fits for the wagering criteria were $-376,-389$ and 393 , respectively.

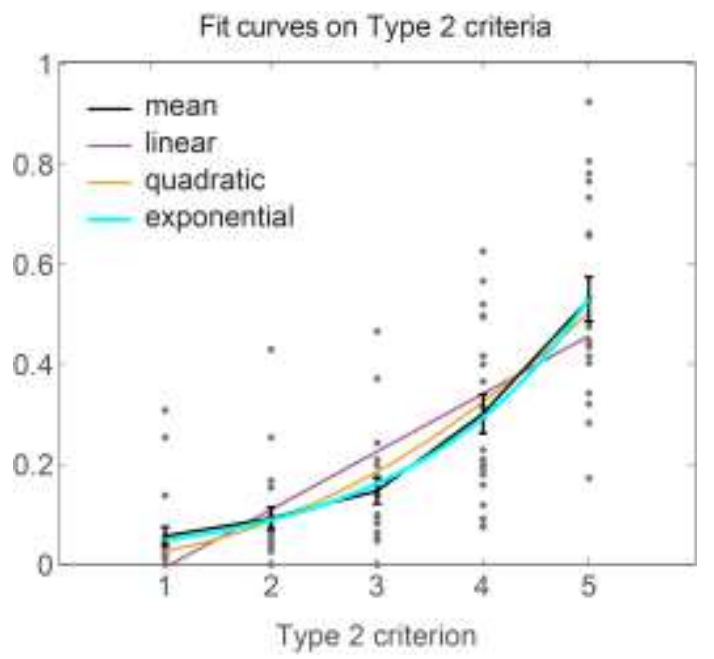

Supplementary Figure 2. Fit curves (linear, quadratic and exponential), subjects' means (dots) and group means and standard errors (black curve) of subjects' wagering criteria. 
Linear model for unidirectional certainty. Although linear models for unidirectional certainty are not able to differentiate certainty of being correct from certainty of being incorrect, they are suitable to experiments which aim at locating areas encoding only one certainty direction and, therefore, have been used in the main studies about confidence evaluation (e.g. Fleming et al., 2012; Hebart et al., 2014 and Heereman et al., 2015). In order to test if our data could be better explained by a linear relationship between wagering and brain activity, we created a parametricGLM with the modulator of the wagering regressor reflecting a linear increase or decrease in BOLD signal (Linear certainty model; Supplementary Fig. 3A). We also created additional post-hoc tests for the areas located using this model. These additional tests required significant differences (paired t-test, $\mathrm{p}<0.05$ ) between the non-parametric beta values of wager 1 and 3 as well as 6 and 4, both in the same direction (Supplementary Fig. 3B). Since both differences should occur in the same direction, we also created a more relaxed criterion that tested only one tail of the t-test (Supplementary Fig. 3C).

A

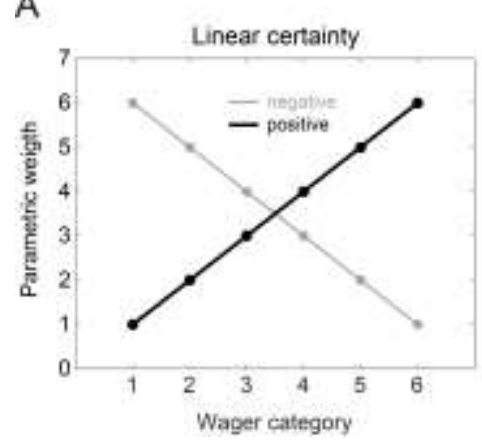

B

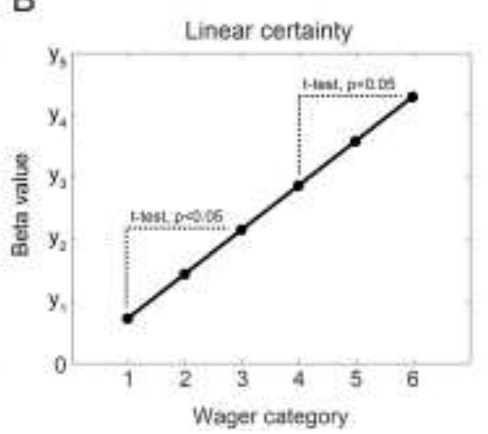

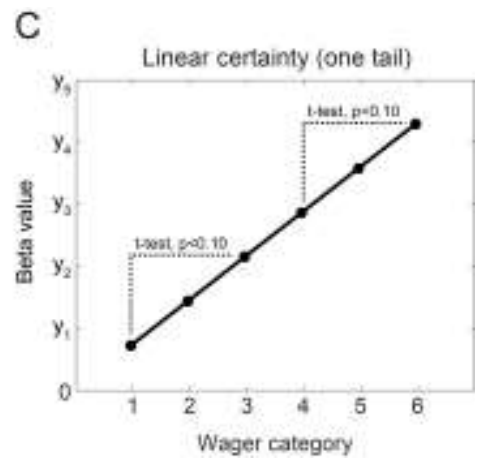

C

Supplementary Figure 3. (A) Parametric weights used for the wagering predictor in the model Linear certainty. (B) Corresponding post-hoc tests applied to guarantee that the predicted brain activity patterns prevailed also for intersubject averages comparisons for two tailed paired t-test and (C) one tailed paired t-test.

When no additional post-hoc tests were applied to the clusters, all the 31 clusters of voxels present in the certainty linear map (Supplementary Fig. 4) shared at least 11 functional voxels with one of the clusters present in the certainty of being correct map. 


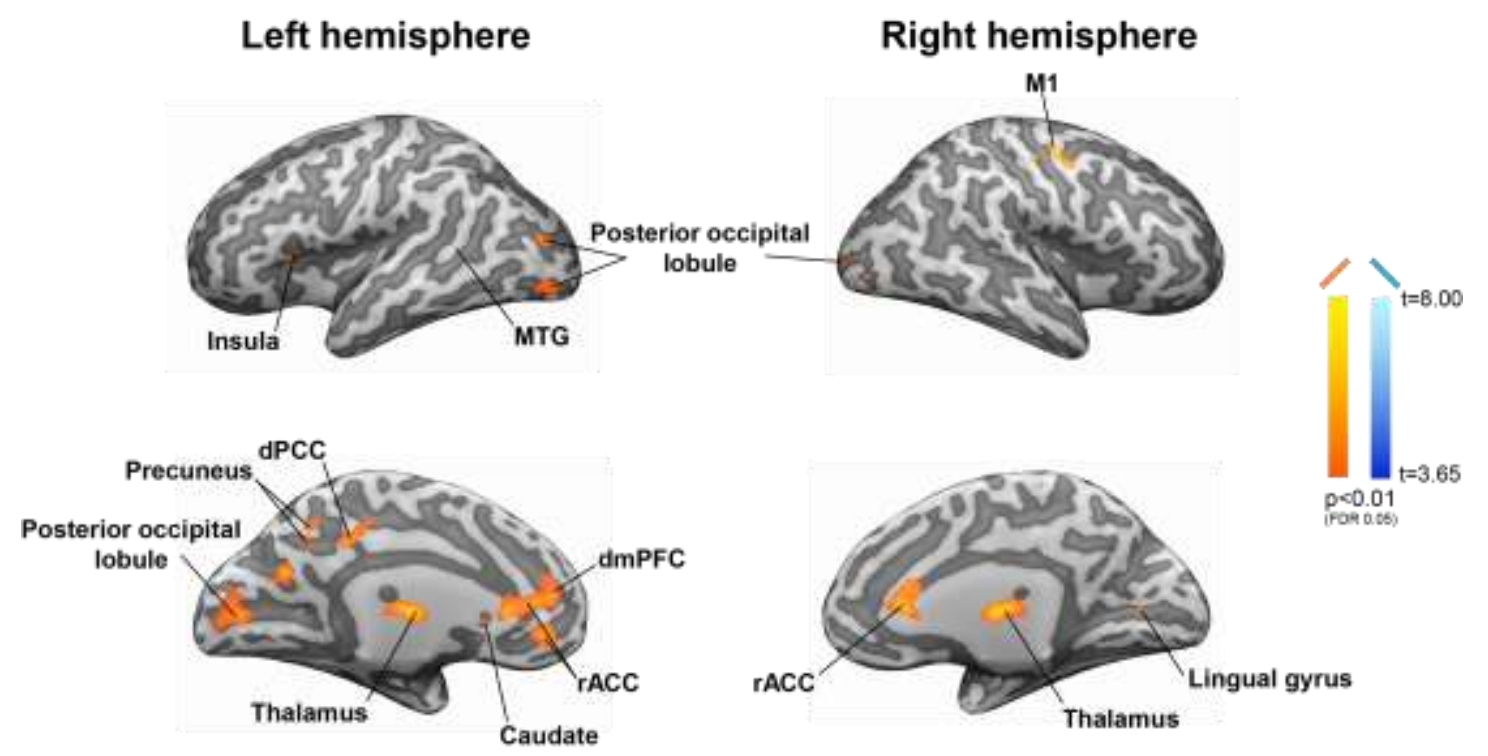

Supplementary Figure 4. Map on inflated brain of cortical areas whose activity varied parametrically according to the "Linear certainty" regressor (no additional post-hoc tests applied).

From the initial 31 clusters of the certainty linear map, only 3 survived the additional post-hoc two tailed t-tests, while 5 survived the one tailed t-tests. On the other hand, 21 clusters survived the post-hoc tests created for the certainty of being correct map (Fig. 4A). These results reveal that most of the clusters of both maps (Certainty linear and certainty of being correct) presented indeed a unidirectional exponential relationship between wager-specific activities. These results also provide support for using wager-specific proportions of correct and incorrect trials as the reference behavioral measure for the modulation of our wagering-related parametric regressors. More importantly, these results demonstrate that subjects were using the wagers nonlinearly to report their certainties.

Pre-experiment. Since wager-specific proportions of correct and incorrect trials cannot predict, by their own, if subjects were reading out both certainty of being correct and certainty of being incorrect, we first confirmed that subjects were able to wager adaptively by significantly reading out both certainty directions using Moreira et al. (2016) task. This task contains a baseline condition (pre-decision wagering, PreDW) that allows the isolation and quantification of PDW trial-specific readouts for both certainty directions independently.

Specifically, Moreira et al. (2016) calculated certainty readouts by subtracting the linear fit slopes of wager-specific proportions of PreDW trials from the linear fit 
slopes of wager-specific proportions of PDW trials. This calculation was done separately for correct (slope-correct) and incorrect (slope-incorrect) trials, generating slope-based measures of certainty of being correct and certainty of being incorrect, respectively. Slope-correct was different between PDW (blue line and band; Supplementary Fig. 5B) and PreDW (blue line and shaded band; Supplementary Fig. $5 \mathrm{~A})$ trials for the 20 subjects of the present study $\left(\mathrm{t}_{19}=3.700, \mathrm{p}<0.05\right)$. Slope-incorrect was also different between PDW and PreDW trials (red line and shaded band, $\mathrm{t}_{19}=-$ 4.438, $\mathrm{p}<0.001$ ). The differences between PreDW and PDW slopes (Supplementary Fig. 5C) confirmed that subjects were able to read out certainty in a bi-directional way.
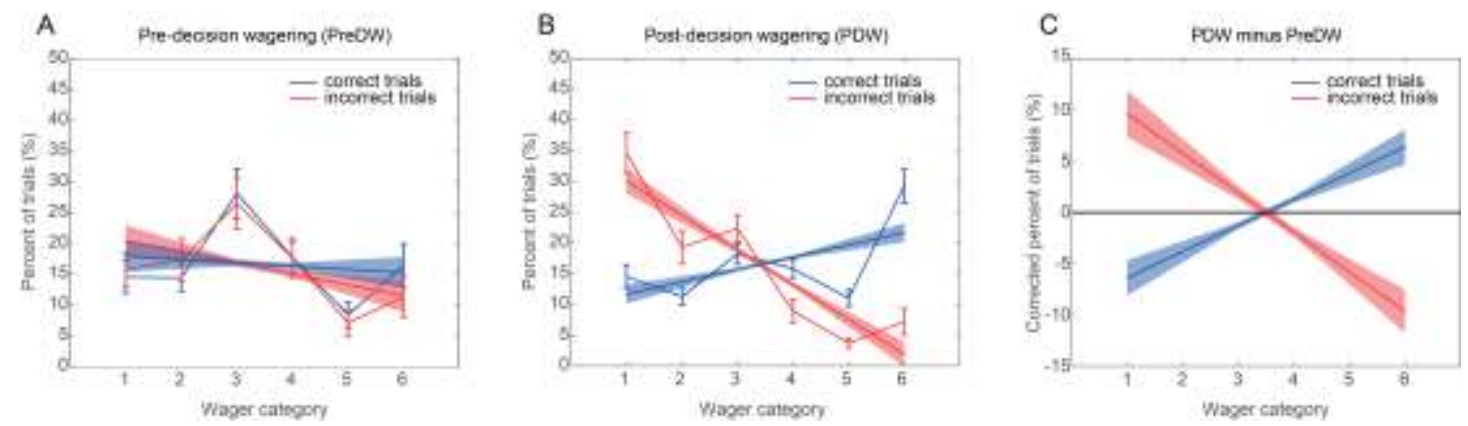

Supplementary Figure 5. Means and standard errors of wagers proportion of correct (blue curve) and incorrect (red curve) trials averaged across subjects, and means and standard errors of slope-correct (blue line and band) and slope-incorrect (red line and band) for (A) PDW and (B) PreDW (baseline). (C) Means and standard errors of PDW slope-correct minus PreDW slope-correct (blue line and band) and of PDW slope-incorrect minus PreDW slope-incorrect (red line and band). All measurements represent averages across difficulty levels and across subjects.

Subjects improved their metacognitive efficiency in the present fMRI experiment $($ meta-d'/d' $=1.57)$ compared to the pre-experiment $\left(\right.$ meta-d' $/ \mathrm{d}^{\prime}=1.08 ; \mathrm{t}_{19}=2.189$, $\mathrm{p}<0.05$ ), indicating that they learned how to perform better metacognitive judgments and/or used the longer interval between the perceptual and the metacognitive decisions in order to accumulate more information for wagering adaptively. Since metacognitive efficiency (meta-d'/d') is also a measure of the metacognitive sensitivity corrected by the sensitivity on the perceptual decisions (Maniscalco and Lau, 2012), the learning component related to the delayed match-to-sample task was not a confounding factor. 
Maps without additional post-hoc tests. Almost two thirds (78 clusters) of the clusters of voxels present in the original bi-directional certainty map (Supplementary Fig. 6) did not survive the additional post-hoc tests for bi-directionality.

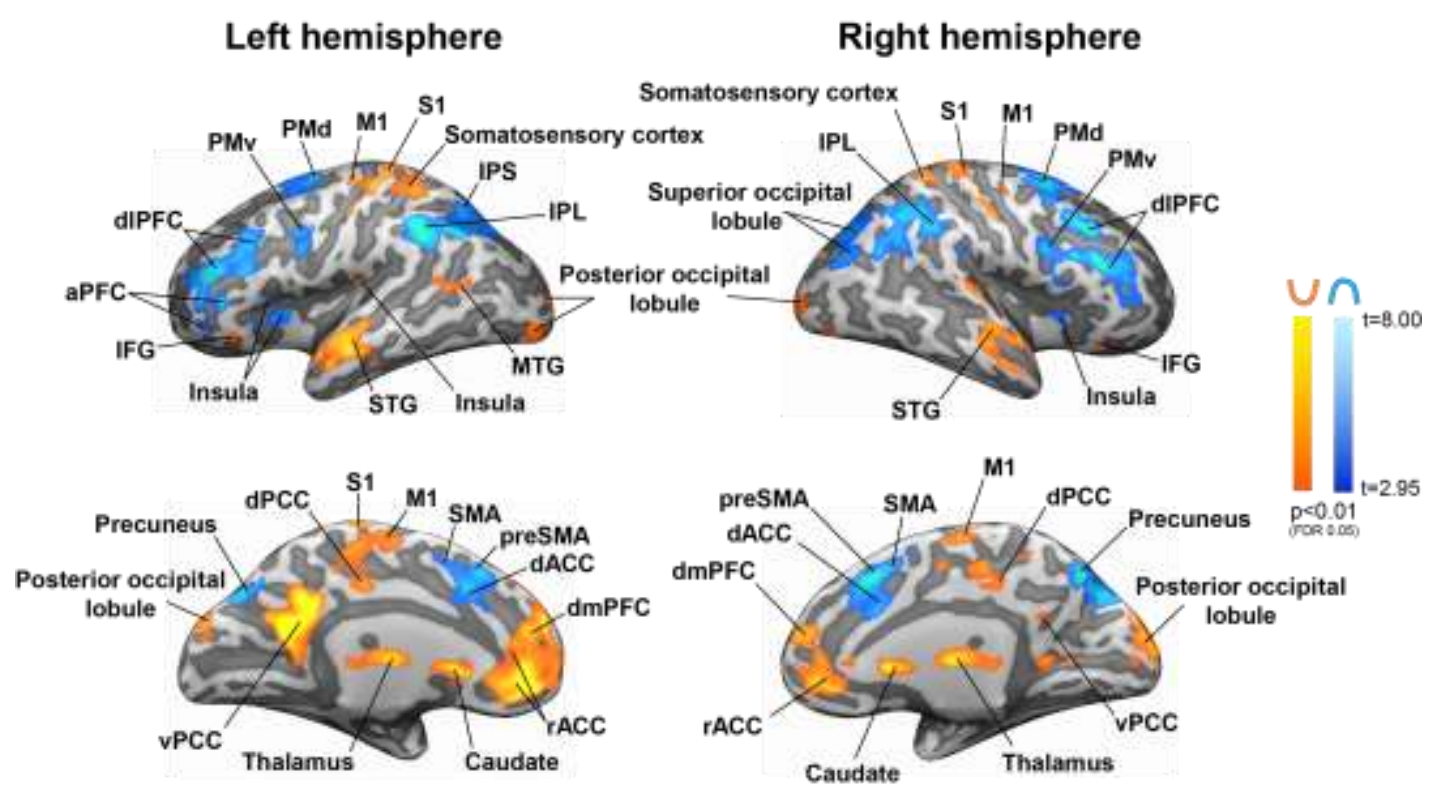

Supplementary Figure 6. Map on inflated brain of cortical areas whose activity varied parametrically according to the "bi-directional certainty" regressor of the wagering event (no additional post-hoc tests applied).

69 clusters where present in the certainty of being correct map before the additional post-hoc tests (Supplementary Fig. 7). Most of them (47 clusters) were still present in the final map (Fig. 10).

\section{Left hemisphere}
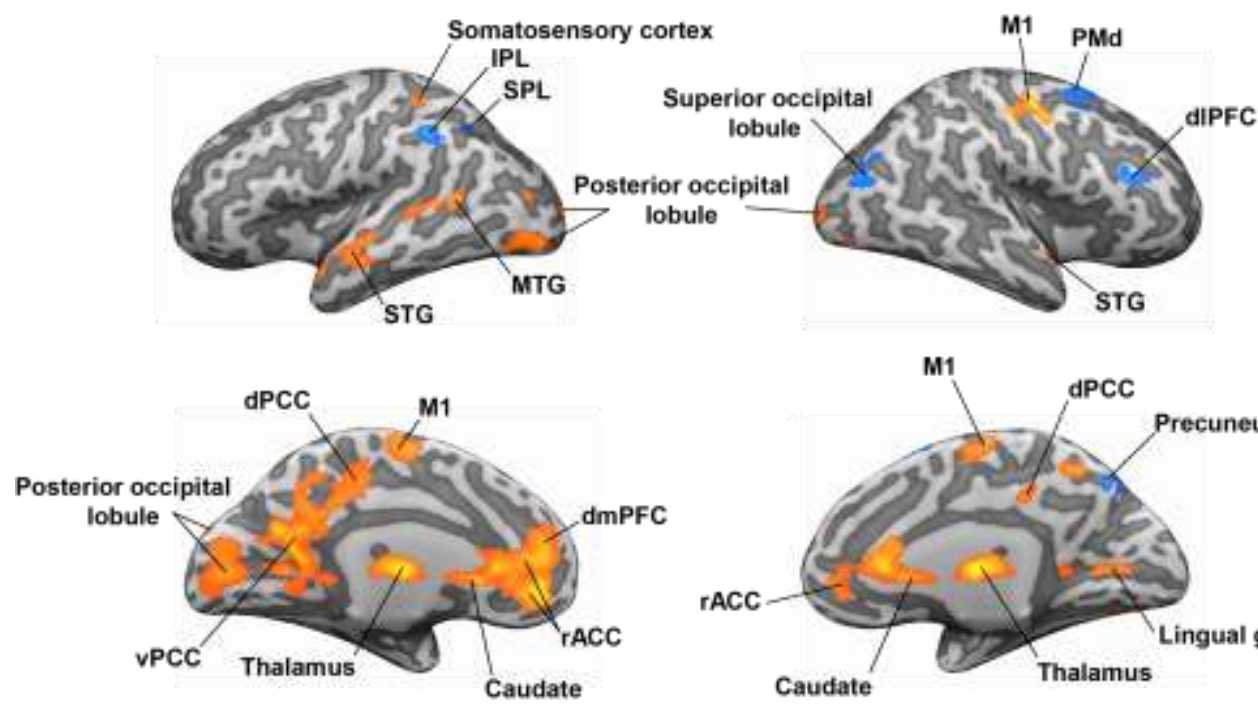

Right hemisphere

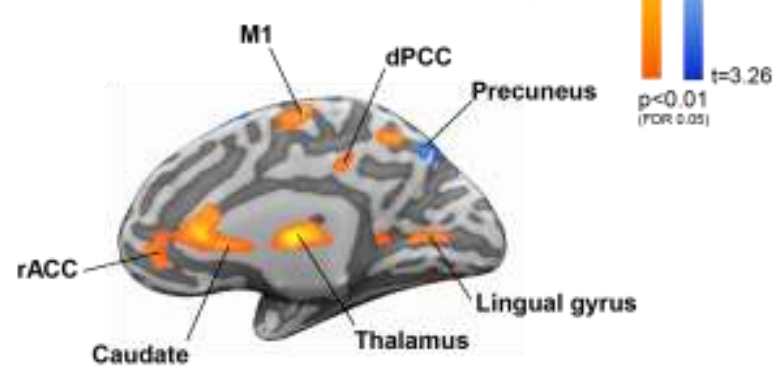


Supplementary Figure 7. Map on inflated brain of cortical areas whose activity varied parametrically according to the "Certainty of being correct" regressor of the wagering event (no additional post-hoc tests applied).

The initial certainty of being incorrect map (Supplementary Fig. 8) was the most affected by the additional post-hoc tests since only $23 \%$ of the 30 clusters survived these tests.

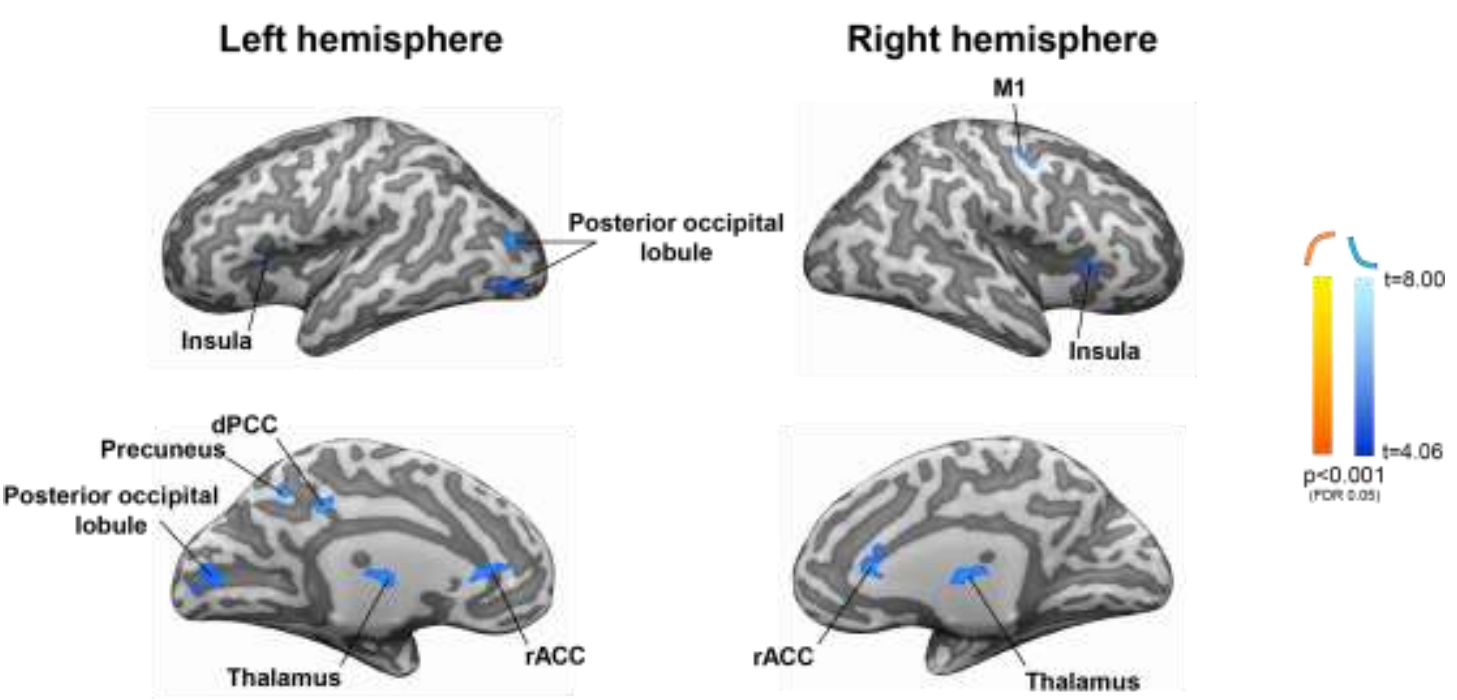

Supplementary Figure 8. Map on inflated brain of cortical areas whose activity varied parametrically according to the "Certainty of being incorrect" regressor of the wagering event (no additional post-hoc tests applied).

When we plot the three non-additionally-corrected maps in the same inflated brain, we can see that there are several overlapping areas among them (Supplementary Fig. 9). 

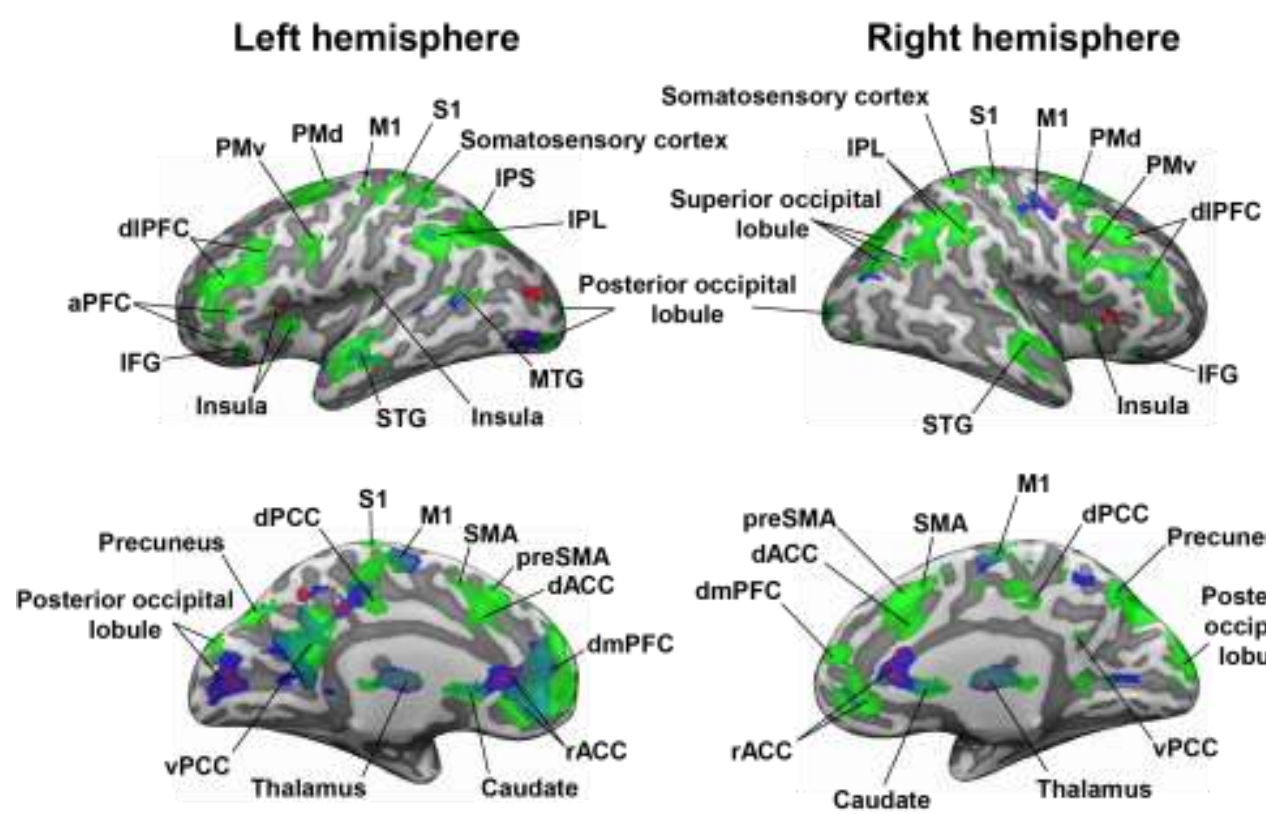

Right hemisphere

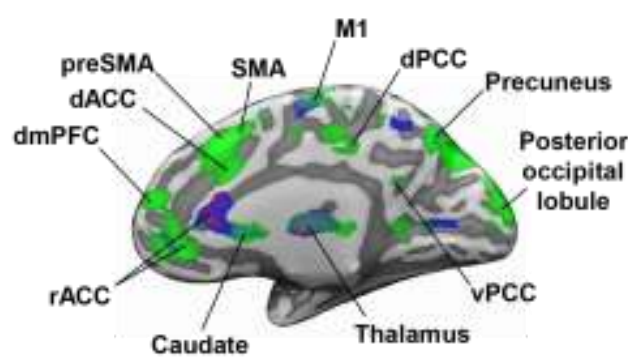

Supplementary Figure 9. Maps on inflated brain of cortical areas whose activity varied parametrically according to the bi-directional certainty (green), certainty of being correct (blue) and certainty of being incorrect (red) regressors of the wagering event (no additional post-hoc tests applied).

These overlaps indicate that the parametric regressors were not selective enough. For example, the overlap between the bi-directional certainty map and the other maps might indicate that the unidirectional models did not restrict their significance to areas with low variance on low wagers (Certainty of being correct function) or low variance on high wagers (Certainty of being incorrect function). It might also indicate that the bi-directional model did not restrict its significance to bi-directional activations patterns, being also influenced by unidirectional activations. Some areas overlapped even for models with opposite predictions (Certainty of being correct and certainty of being incorrect). In this context, we demonstrated that the use of the additional posthoc tests was essential to locate areas encoding specific certainty-related patterns.

"Hebart's area" for the degree of perceptual confidence. Herbart et al. (2014) suggested that the activity in the ventral striatum reflects confidence, being derived from a transformation of the decision variable encoded in the cerebral cortex. In the present experiment, the ventral striatum encoded difficulty levels (first row of the Supplementary Fig. 10).

Although this area was not present in our certainty of being correct map, we extracted the beta values for the certainty of being correct parametric regressor (wagering 
event) and applied the additional post-hoc tests related to its model. On both hemispheres, the activity increased in high wagers, while it did not vary in low wagers (second row of the Supplementary Fig. 10).
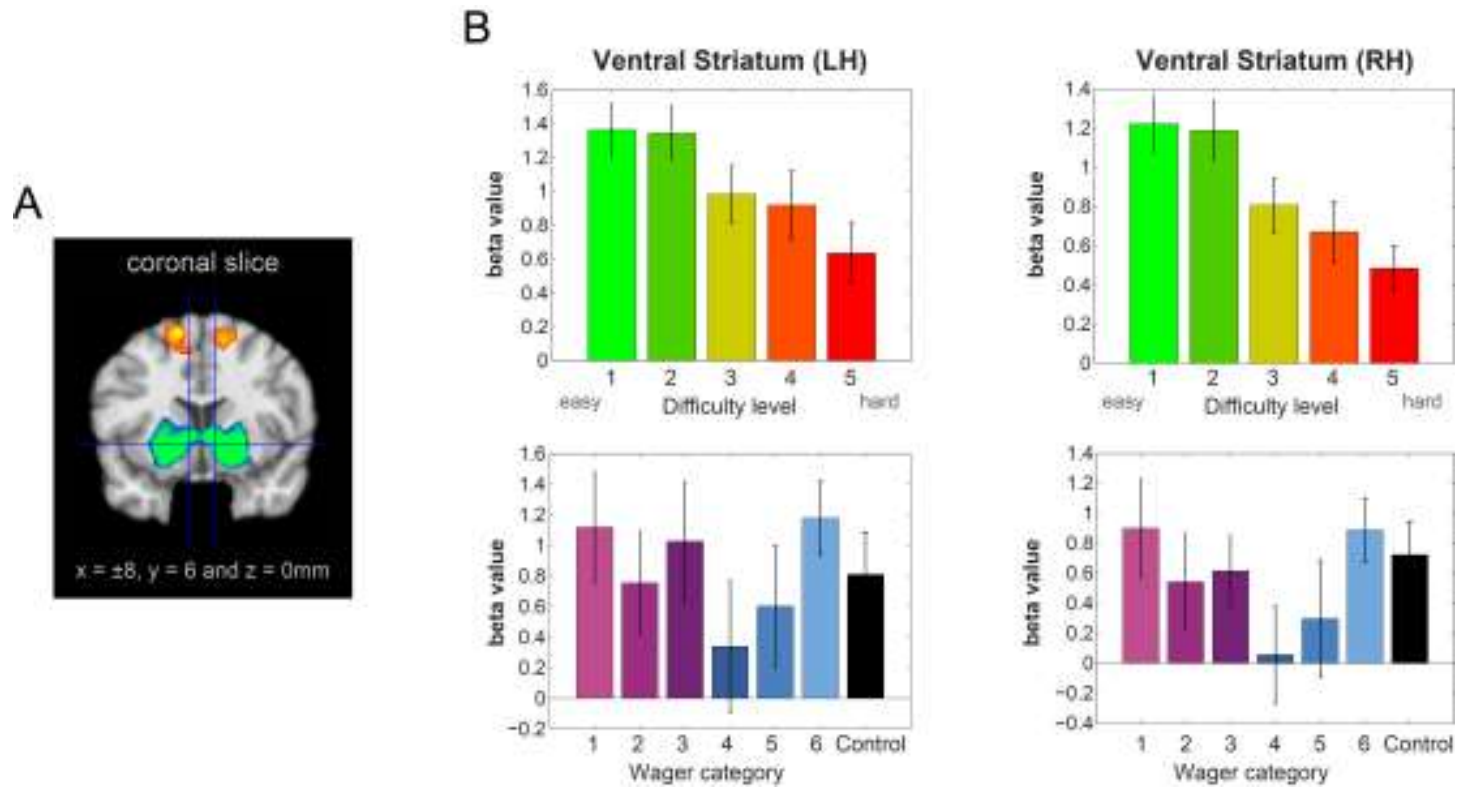

Supplementary Figure 10. (A) Coronal slice encompassing the ventral striatum of Hebart et al. (2014; Talairach coordinates: $x= \pm 8 \mathrm{~mm}, \mathrm{y}=6 \mathrm{~mm}$ and $\mathrm{z}=0 \mathrm{~mm}$ ). (B) Beta value means and standard errors for each of the five difficulty levels (first row) or each of the six wagers (second row) non-parametric regressors of the ventral striatum (left and right columns for the left and right hemispheres, respectively). Difficulty varies from the easiest level (level 1, light green) to the hardest level (level 5, red), and wagers are presented from the smallest stack (category 1, pink) to the largest stack (category 6 , light blue). The activity related to control trials (instructed wagering) is represented in the black bar.

Overlap between the main maps. Part of the ventral Posterior Cingulate Cortex (vPCC) was present in the bi-directional certainty and the certainty of being correct maps. The average beta values extracted from the overlapping voxels for the wagers 1 and 3 were not statistically different $\left(\mathrm{t}_{19}=1.8268, \mathrm{p}=0.08\right)$, while the average beta values of wagers 4 and 6 significantly differed $\left(\mathrm{t}_{19}=-4.431, \mathrm{p}<0.001\right.$; Supplementary Fig. 11). This result indicates that the overlapping voxels can only be classified as belonging to the certainty of being correct map. The close to significance difference between the estimates of wagers 1 and 3 indicates that part of vPCC is likely to be involved in bi-directional certainty readouts, as the area belonging to the bidirectional certainty map above the overlapping voxels (Fig. 13). 
A

Left hemisphere

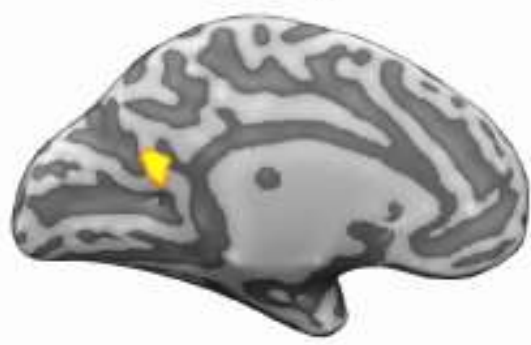

B

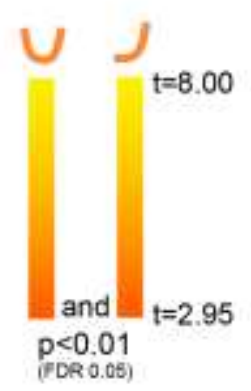

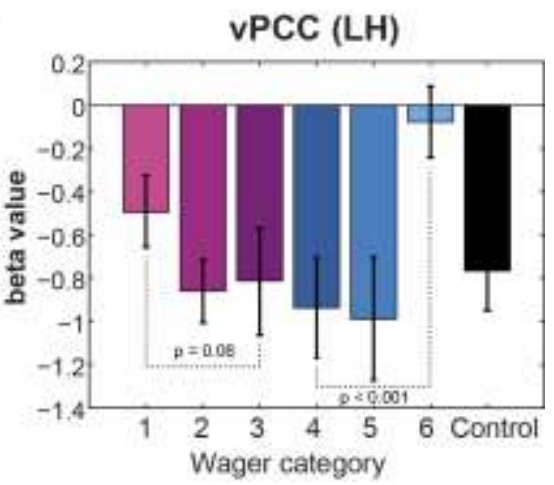

Supplementary Figure 11. (A) Overlapping voxels between bi-directional certainty and certainty of being correct maps. (B) The activity pattern of vPCC did not survive the additional post-hoc test for bidirectionality in the left branch (wager categories 1 and 3). Wagers are presented from the smallest stack (category 1, pink) to the largest stack (category 6 , light blue). The activity related to control trials (instructed wagering) is represented in the black bar.

Wager-specific proportions of all trials. Wager-specific proportions of correct trials were calculated as the "number of correct trials each wager was assigned to" divided by "all correct trials"; and wager-specific proportions of incorrect trials were calculated as the "number of incorrect trials each wager was assigned to" divided by "all incorrect trials". Wager-specific proportion of all trials reflected, therefore, the frequency of the use of a certain wager. Wagers were used in different frequencies $\left(F_{5,95}=33.689\right.$, $p<0.0001$; Supplementary Fig. 12). Pots-hoc Bonferroni tests showed that, when considering all trials, the three low wagers were used with the same frequency $(p>0.05)$, while all the wagers were used less frequently than the wager 6 $(\mathrm{p}<0.05)$. The wager 5 was used less frequently than the wager $1(\mathrm{p}<0.05)$.

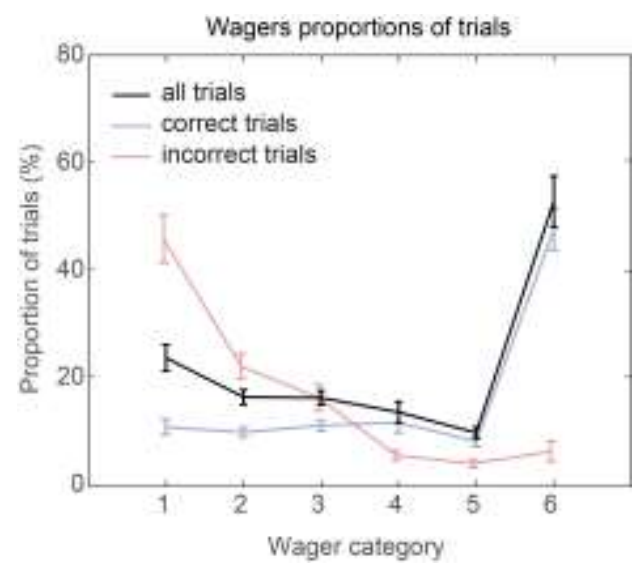

Supplementary Figure 12. Wager-specific proportions of all trials (black curve). The red curve represents the wager-specific proportions of incorrect trials. The blue curve represents the wagerspecific proportions of correct trials. 
"Fleming's area" for confidence. Among the studies with humans aiming to locate brain areas encoding certainty of being correct, Fleming et al. (2012) is one of the most important ones since it showed that, during their experiment, the activity in the right anterior prefrontal cortex (aPFC) varied parametrically according to certainty of being correct and correlated with subjects' metacognitive sensitivity. Although Fleming's area was not present in any of our maps, we extracted its beta values to check its activity pattern for subjects performing our task. Interestingly, the right anterior prefrontal cortex (aPFC, Talairach coordinates $\mathrm{x}=26 \mathrm{~mm}, \mathrm{y}=52 \mathrm{~mm}$ and $\mathrm{z}=23 \mathrm{~mm}$ ) had a similar pattern described by Fleming et al. (2012), with decreasing activity for higher certainty of being correct $\left(t_{19}=2.261, p<0.05\right.$; Supplementary Fig. 13). Also as in Fleming et al. (2012), the same area in the left hemisphere did not present such pattern significantly.

The absence of the "Fleming's area" in the unidirectional certainty of being correct map indicates, however, that on average (across subjects) this pattern is not consistent with the pattern predicted by the certainty of being correct model, possibly because the beta values of the low wagers are more similar to the beta value of the highest wager than to the beta values of the wager 4 , while the certainty of being correct model predicts increasing variance towards the wager 6 .
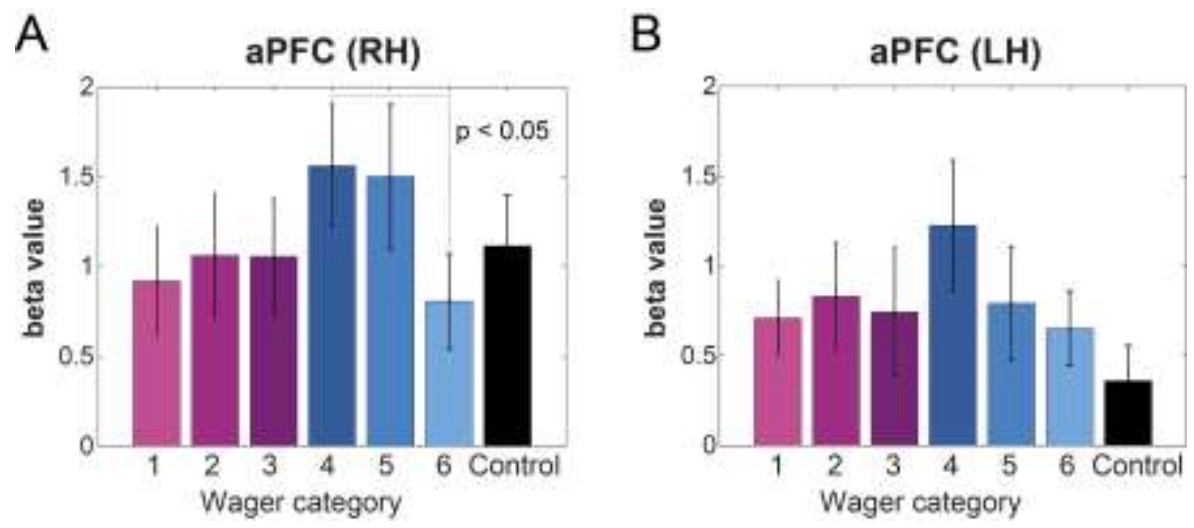

Supplementary Figure 13. Beta value means and standard errors of non-parametric regressors of wager categories and control trials for the area located by Fleming et al. (2012): (A) right aPFC and (B) the area located in similar coordinates in the left hemisphere. Wagers are presented from the smallest stack (category 1, pink) to the largest stack (category 6, light blue). The activity related to control trials (instructed wagering) is represented in the black bar. 
Wager-specific proportions of trials in the five difficulty levels. Although we were not able to quantify certainty of being correct and certainty of being incorrect readouts without the baseline condition used in Moreira et al. (2016), we know that if subjects would could not distinguish correct from incorrect perceptual decisions, they would assign wagers randomly to correct and incorrect trials, generating wager-specific proportions of correct trials similar to the wager-specific proportions of incorrect trials and, consequently, similar slope-correct and slope-incorrect values (e.g. Supplementary Fig. 5A). Since slope-correct was different from slope-incorrect in all difficulty levels ( $p<0.001$ for the five paired t-tests), we demonstrated that subjects were able to differentiate correct and incorrect perceptual decisions from all difficulties, independently on the certainty readout they were using (certainty of being correct or certainty of being incorrect). As expected, the difference between slopecorrect and slope-incorrect decreased on harder trials $\left(\mathrm{F}_{4,76}=45.169, \mathrm{p}<0.0001\right.$; Supplementary Fig. 14).
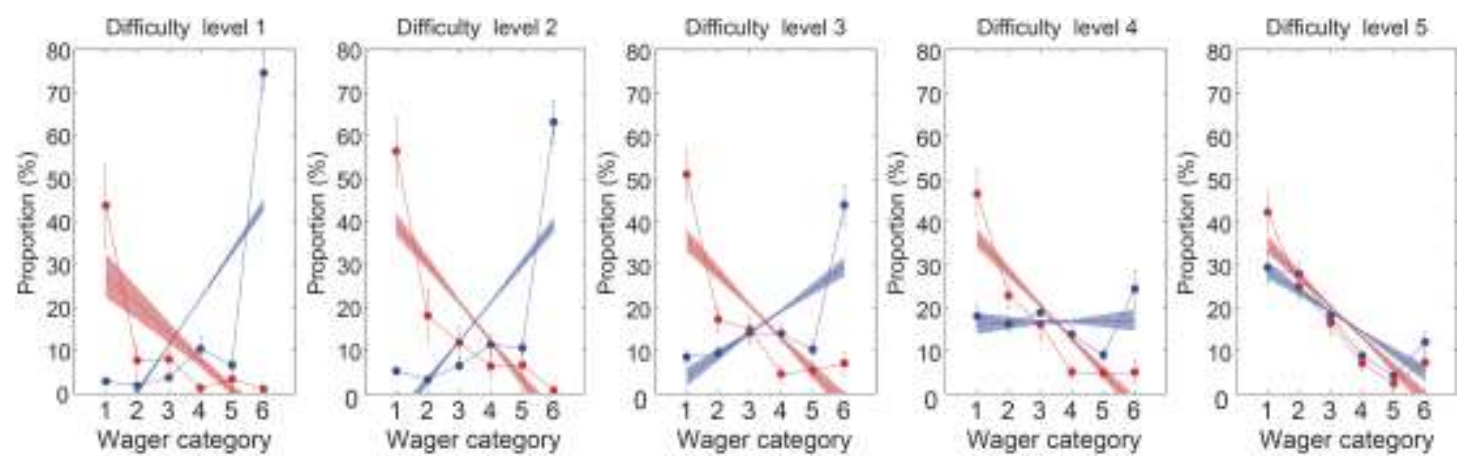

Supplementary Figure 14. For each difficulty level, means and standard errors of the wagers proportion of correct (blue curves) and incorrect (red curves) trials, as well as means and standard errors of slopecorrect (blue line and band) and slope-incorrect (red line and band). See the section Pre-experiment of the SI Results for more information about the measures slope-correct and slope-incorrect. All measures averaged across subjects.

It is important to emphasize here that, by using the slope-based measurements, we were not trying to fit the best curve that explains the variation in wager-specific proportions of correct and incorrect trials (which, as described in the Supplementary Information section: Linear model for unidirectional certainty, are on average best described by an exponential function). Instead, we aimed to compare subjects' trends on assigning wagers to correct and incorrect decisions (see Chapter 1). 


\section{References}

Agam, Y., Hamalainen, M. S., Lee, A. K. C., Dyckman, K. A., Friedman, J. S., Isom, M., ... Manoach, D. S. (2011). Multimodal neuroimaging dissociates hemodynamic and electrophysiological correlates of error processing. Proceedings of the National Academy of Sciences, 108(42), 17556-17561. http://doi.org/10.1073/pnas.1103475108

Baird, B., Smallwood, J., Gorgolewski, K. J., \& Margulies, D. S. (2013). Medial and Lateral Networks in Anterior Prefrontal Cortex Support Metacognitive Ability for Memory and Perception. Journal of Neuroscience, 33(42), 16657-16665. http://doi.org/10.1523/JNEUROSCI.0786-13.2013

Burnham, K. P. (2004a). Multimodel Inference: Understanding AIC and BIC in Model Selection. Sociological Methods \& Research, 33(2), 261-304. http://doi.org/10.1177/0049124104268644

Burnham, K. P. (2004b). Multimodel Inference: Understanding AIC and BIC in Model Selection. Sociological Methods \& Research, 33(2), 261-304. http://doi.org/10.1177/0049124104268644

Chakraborty, S., Kolling, N., Walton, M. E., \& Mitchell, A. S. (2016). Critical role for the mediodorsal thalamus in permitting rapid reward-guided updating in stochastic reward environments. eLife, 5, e13588.

Charles, L., Van Opstal, F., Marti, S., \& Dehaene, S. (2013). Distinct brain mechanisms for conscious versus subliminal error detection. NeuroImage, 73, 80-94. http://doi.org/10.1016/j.neuroimage.2013.01.054

Cisek, P., \& Kalaska, J. F. (2005). Neural Correlates of Reaching Decisions in Dorsal Premotor Cortex: Specification of Multiple Direction Choices and Final Selection of Action. Neuron, 45(5), 801-814. http://doi.org/10.1016/j.neuron.2005.01.027

De Martino, B., Fleming, S. M., Garrett, N., \& Dolan, R. J. (2012). Confidence in value-based choice. Nature Neuroscience, 16(1), 105-110. http://doi.org/10.1038/nn.3279

Festinger, L. (1943). Studies in decision: I. Decision-time, relative frequency of judgment and subjective confidence as related to physical stimulus difference. Journal of Experimental Psychology, 32(4), 291. 
Field, A. P. (2009). Discovering statistics using SPSS: (and sex, drugs and rock "n" roll) (3rd ed). Los Angeles: SAGE Publications.

Filimon, F., Philiastides, M. G., Nelson, J. D., Kloosterman, N. A., \& Heekeren, H. R. (2013). How Embodied Is Perceptual Decision Making? Evidence for Separate Processing of Perceptual and Motor Decisions. Journal of Neuroscience, 33(5), 2121-2136. http://doi.org/10.1523/JNEUROSCI.2334-12.2013

Fleming, S. M. (2016). Changing our minds about changes of mind. Elife, 5, e14790.

Fleming, S. M., Huijgen, J., \& Dolan, R. J. (2012). Prefrontal Contributions to Metacognition in Perceptual Decision Making. Journal of Neuroscience, 32(18), 6117-6125. http://doi.org/10.1523/JNEUROSCI.6489-11.2012

Fleming, S. M., \& Lau, H. C. (2014). How to measure metacognition. Frontiers in Human Neuroscience, 8. http://doi.org/10.3389/fnhum.2014.00443

Fleming, S. M., Maniscalco, B., Ko, Y., Amendi, N., Ro, T., \& Lau, H. (2015). Action-specific disruption of perceptual confidence. Psychological Science, 26(1), 89-98.

Haber, S. N. (2003). The primate basal ganglia: parallel and integrative networks. Journal of Chemical Neuroanatomy, 26(4), 317-330. http://doi.org/10.1016/j.jchemneu.2003.10.003

Hebart, M. N., Schriever, Y., Donner, T. H., \& Haynes, J.-D. (2016). The Relationship between Perceptual Decision Variables and Confidence in the Human Brain. Cerebral Cortex, 26(1), 118-130. http://doi.org/10.1093/cercor/bhu181

Heekeren, H. R., Marrett, S., \& Ungerleider, L. G. (2008). The neural systems that mediate human perceptual decision making. Nature Reviews Neuroscience, 9(6), 467-479. http://doi.org/10.1038/nrn2374

Heereman, J., Walter, H., \& Heekeren, H. R. (2015). A task-independent neural representation of subjective certainty in visual perception. Frontiers in Human Neuroscience, 9. http://doi.org/10.3389/fnhum.2015.00551

Hochman, E. Y., Vaidya, A. R., \& Fellows, L. K. (2014). Evidence for a Role for the Dorsal Anterior Cingulate Cortex in Disengaging from an Incorrect Action. PLoS ONE, 9(6), e101126. http://doi.org/10.1371/journal.pone.0101126

Kepecs, A., \& Mainen, Z. F. (2012). A computational framework for the study of confidence in humans and animals. Philosophical Transactions of the Royal 
Society B: Biological Sciences, 367(1594), 1322-1337. http://doi.org/10.1098/rstb.2012.0037

Kepecs, A., Uchida, N., Zariwala, H. A., \& Mainen, Z. F. (2008). Neural correlates, computation and behavioural impact of decision confidence. Nature, 455(7210), 227-231. http://doi.org/10.1038/nature07200

Kerns, J. G. (2004). Anterior Cingulate Conflict Monitoring and Adjustments in Control. Science, 303(5660), 1023-1026. http://doi.org/10.1126/science.1089910

Kiani, R., Corthell, L., \& Shadlen, M. N. (2014). Choice Certainty Is Informed by Both Evidence and Decision Time. Neuron, 84(6), 1329-1342. http://doi.org/10.1016/j.neuron.2014.12.015

Kiani, R., \& Shadlen, M. N. (2009). Representation of Confidence Associated with a Decision by Neurons in the Parietal Cortex. Science, 324(5928), 759-764. http://doi.org/10.1126/science.1169405

Komura, Y., Nikkuni, A., Hirashima, N., Uetake, T., \& Miyamoto, A. (2013). Responses of pulvinar neurons reflect a subject's confidence in visual categorization. Nature Neuroscience, 16(6), 749-755. http://doi.org/10.1038/nn.3393

Ma, W. J., \& Jazayeri, M. (2014). Neural Coding of Uncertainty and Probability. Annual Review of Neuroscience, 37(1), 205-220. http://doi.org/10.1146/annurev-neuro-071013-014017

Mamassian, P., Landy, M. S., \& Maloney, L. T. (2002). Bayesian modelling of visual perception. Probabilistic Models of the Brain: Perception and Neural Function, $13-36$.

Maniscalco, B., \& Lau, H. (2012). A signal detection theoretic approach for estimating metacognitive sensitivity from confidence ratings. Consciousness and Cognition, 21(1), 422-430. http://doi.org/10.1016/j.concog.2011.09.021

Moran, R., Teodorescu, A. R., \& Usher, M. (2015). Post choice information integration as a causal determinant of confidence: Novel data and a computational account. Cognitive Psychology, 78, 99-147. http://doi.org/10.1016/j.cogpsych.2015.01.002

Moreira, C. M., Rollwage, M., Kaduk, K., Wilke, M., Kagan, I. (2016). Certainty bidirectionality and the efficient use of metacognition. Submitted to Cognition. 
Murphy, P. R., Robertson, I. H., Harty, S., \& O’Connell, R. G. (2015). Neural evidence accumulation persists after choice to inform metacognitive judgments. eLife, 4. http://doi.org/10.7554/eLife.11946

O’Doherty, J. (2004a). Dissociable Roles of Ventral and Dorsal Striatum in Instrumental Conditioning. Science, 304(5669), 452-454. http://doi.org/10.1126/science.1094285

O'Doherty, J. (2004b). Dissociable Roles of Ventral and Dorsal Striatum in Instrumental Conditioning. Science, 304(5669), 452-454. http://doi.org/10.1126/science.1094285

Persaud, N., McLeod, P., \& Cowey, A. (2007). Post-decision wagering objectively measures awareness. Nature Neuroscience, 10(2), 257-261. http://doi.org/10.1038/nn1840

Pleskac, T. J., \& Busemeyer, J. R. (2010). Two-stage dynamic signal detection: A theory of choice, decision time, and confidence. Psychological Review, 117(3), 864-901. http://doi.org/10.1037/a0019737

Pouget, A., Drugowitsch, J., \& Kepecs, A. (2016). Confidence and certainty: distinct probabilistic quantities for different goals. Nature Neuroscience, 19(3), 366374. http://doi.org/10.1038/nn.4240

Ratcliff, R., \& McKoon, G. (2008). The diffusion decision model: theory and data for two-choice decision tasks. Neural Computation, 20(4), 873-922.

Ridderinkhof, K. R., van den Wildenberg, W. P. M., Segalowitz, S. J., \& Carter, C. S. (2004). Neurocognitive mechanisms of cognitive control: The role of prefrontal cortex in action selection, response inhibition, performance monitoring, and reward-based learning. Brain and Cognition, 56(2), 129-140. http://doi.org/10.1016/j.bandc.2004.09.016

Rolls, E. T., Grabenhorst, F., \& Deco, G. (2010). Choice, difficulty, and confidence in the brain. $\quad$ NeuroImage, 53(2), 694-706. http://doi.org/10.1016/j.neuroimage.2010.06.073

Rounis, E., Maniscalco, B., Rothwell, J. C., Passingham, R. E., \& Lau, H. (2010). Theta-burst transcranial magnetic stimulation to the prefrontal cortex impairs metacognitive visual awareness. Cognitive neuroscience, 1(3), 165-175.

Seifert, S., von Cramon, D. Y., Imperati, D., Tittgemeyer, M., \& Ullsperger, M. (2011). Thalamocingulate Interactions In Performance Monitoring. Journal of 
Neuroscience, 31(9), 3375-3383. http://doi.org/10.1523/JNEUROSCI.624210.2011

Shadlen, M. N., Kiani, R., Hanks, T. D., \& Churchland, A. K. (2008). An intentional framework. Better than Conscious, 71-101.

Shadlen, M. N., \& Newsome, W. T. (2001). Neural basis of a perceptual decision in the parietal cortex (area LIP) of the rhesus monkey. Journal of Neurophysiology, 86(4), 1916-1936.

Talmi, D., Seymour, B., Dayan, P., \& Dolan, R. J. (2008). Human Pavlovian Instrumental Transfer. Journal of Neuroscience, 28(2), 360-368. http://doi.org/10.1523/JNEUROSCI.4028-07.2008

Taylor, J. E., Ogawa, A., \& Sakagami, M. (2016). Reward value enhances postdecision error-related activity in the cingulate cortex. Neuroscience Research, 107, 38-46. http://doi.org/10.1016/j.neures.2015.12.009

Yeung, N., \& Summerfield, C. (2012). Metacognition in human decision-making: confidence and error monitoring. Philosophical Transactions of the Royal Society B: Biological Sciences, 367(1594), 1310-1321. http://doi.org/10.1098/rstb.2011.0416

Yu, S., Pleskac, T. J., \& Zeigenfuse, M. D. (2015). Dynamics of postdecisional processing of confidence. Journal of Experimental Psychology: General, 144(2), 489-510. http://doi.org/10.1037/xge0000062 


\section{Chapter 3}

Certainty about response requirements influences action selection and interhemispheric processing in monkeys and humans 


\section{Table of Contents}

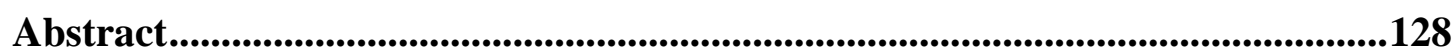

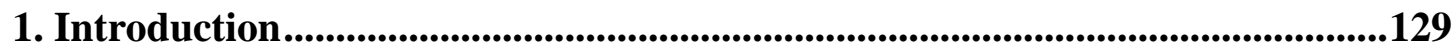

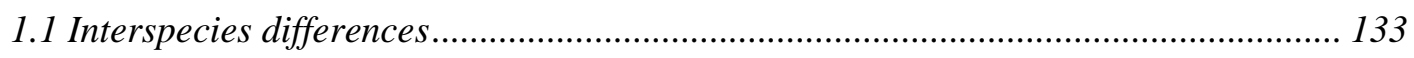

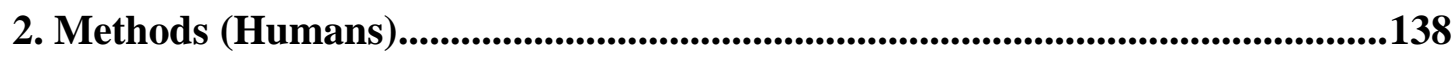

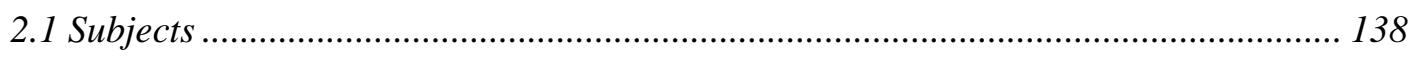

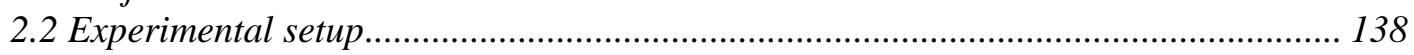

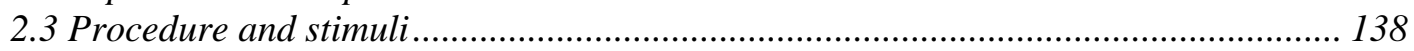

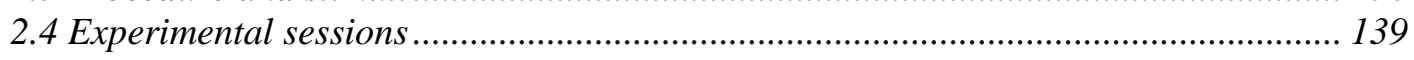

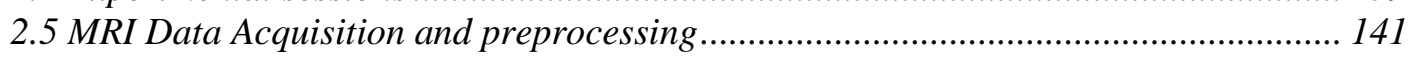

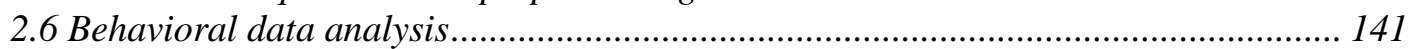

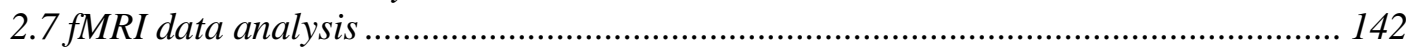

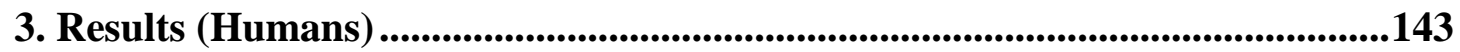

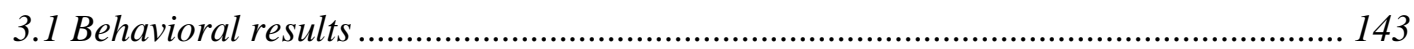

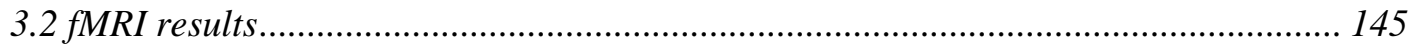

4. Methods (Monkeys) ............................................................................................................152

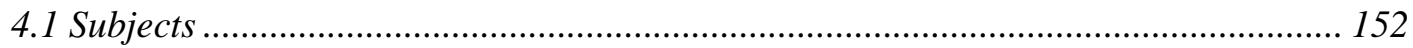

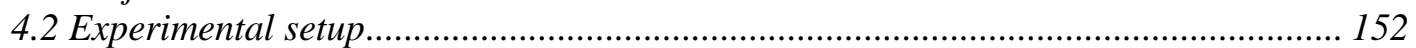

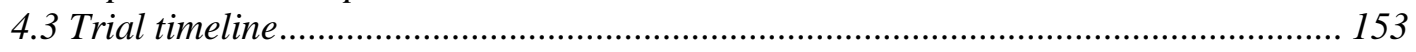

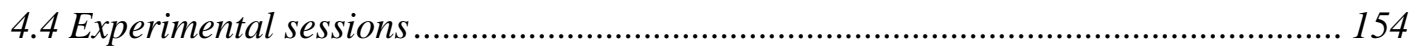

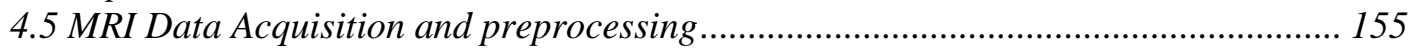

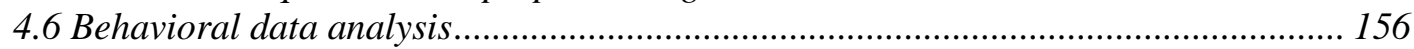

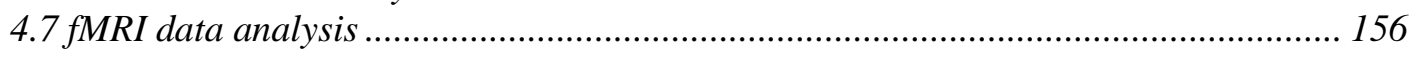

5. Results (Monkeys) ..................................................................................................................157

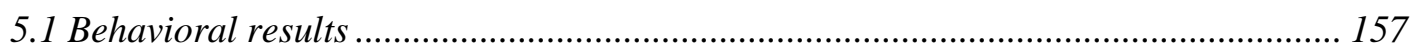

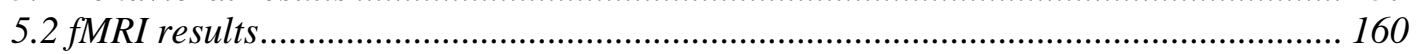

6. Direct interspecies behavioral comparisons ...................................................166

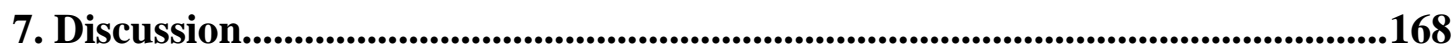

7.1 Interspecies differences in hemispheric specialization ............................................... 170

7.2 Differences in CUD-related activation patterns and RT across the tasks .................. 171

7.3 Poffenberger paradigm vs the NAS task: why we did not find CUD in RT?.............. 173

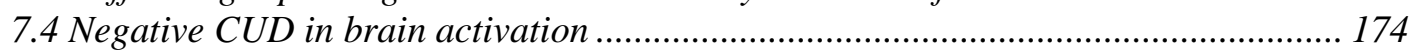

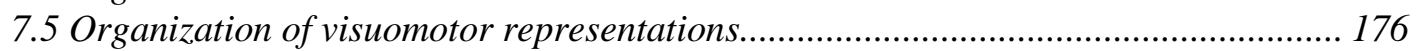

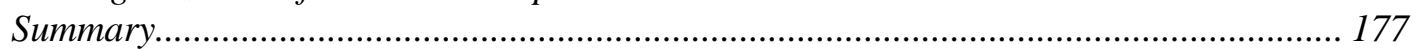

Supplementary information .................................................................................179

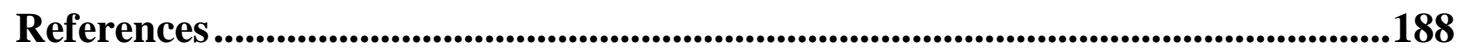




\begin{abstract}
All primates rely on interhemispheric communication to create unified representations of the environment and generate flexible responses, especially when there is conflicting information between the brain hemispheres. Interhemispheric transfer has been studied for more than one century using speeded manual responses to lateralized visual cues (Poffenberger paradigm), but it is still unknown how the predictability of upcoming response requirements modifies this communication. Furthermore, although this paradigm was tested in different contexts in humans, no previous study tested how the interhemispheric transfer of visuomotor information differs between humans and non-human primates. Therefore, fourteen human subjects and two rhesus monkeys performed three tasks with different predictability about the upcoming response requirements inside an MRI scanner while we recorded BOLD signal from their brains. Typically, reaction times are faster for uncrossed responses (which do not require interhemispheric transfer of information) than for crossed responses, resulting in crossed-uncrossed difference (CUD). Both humans and monkeys exhibited reaction time and neural CUD effects, but the size of the effect varied depending on the task and on the species. The increased uncertainty about the upcoming response requirements prolonged apparent interhemispheric transfer time in both species, presumably by adding action selection and stimulus-response spatial compatibility effects to the brain processing. The main difference between the species was that humans showed only positive neural CUD (crossed>uncrossed), while monkeys showed both effects. According to our interpretation of activation patterns in humans, unpredictability did not increase the interhemispheric transfer time per se, but delayed the exchange of relevant information between the hemispheres until the proper response could be implemented without decreasing performance. Shorter transfer times in monkeys suggest that they failed in delaying responses or their smaller brains (and consequently interhemispheric distances) allowed faster interhemispheric communication.
\end{abstract}

Key-words: predictability, interhemispheric communication, action selection, Poffenberger, comparative, rhesus monkeys

Abbreviations: crossed uncrossed difference (CUD), reaction time (RT), stimulusresponse spatial compatibility (SRSC) 


\section{Introduction}

Primate evolution resulted in lateralized brains which process visual and motor information mainly in contralateral fashion. The visual information in the right visual field is first represented in the left primary visual cortex, while the opposite holds for the left visual field. Conversely, the left primary motor cortex encodes mainly right effectors (e.g. right hand and right foot) responses, and vice-versa for the left primary motor cortex. While the exact evolutionary contingencies of these anatomical and functional specializations are very debatable (e.g. Loosemore, 2011; Oleksiak et al., 2010), it is a fact that all living primates need interhemispheric communication to interact adaptively with the environment. For example, without interhemispheric transfer of information, we would not be able to respond with our left hand to objects approaching us from the periphery of our right visual field. More importantly, the unified perception of surroundings is essential for individuals to perform goaldirected behaviors, especially when the information initially represented in one hemisphere is conflicting, or competing, with the information represented in the other hemisphere, for example during spatial choices spanning both visual hemifields (Kagan et al., 2010). The gradual progression from finely topographically organized early visual areas to a coarser, mainly contralateral, topography of parietal and frontal areas (Felleman and Van Essen, 1991) indicates a transformation from the "local" visual processing to a "global" representation of action space. This progression is further influenced by accumulation of inter-hemispheric signals at subsequent stages of processing, leading to more bilateral representations in higher order cortical areas.

The interhemispheric transfer of visuomotor information was first tested by Poffenberger (1912) using a simple reaction time task. Poffenberger calculated interhemispheric transfer time by comparing reaction times when flashes of light were presented in the periphery of subjects' right or left visual field, and they responded with the right or the left hand as soon as they saw the flash. Due to predominantly contralateral processing, when subjects responded with the hand positioned in the same side of the visual stimulus, there was no need for interhemispheric transfer of information (uncrossed response) and, consequently, response time was faster. Crossed responses, however, requires interhemispheric communication and, therefore, were slower (Fig. 1). The time the brain takes to transfer this information was 
calculated by Poffenberger by subtracting the averaged uncrossed from the averaged crossed response times (Crossed Uncrossed Difference, CUD; Poffenberger, 1912).

A

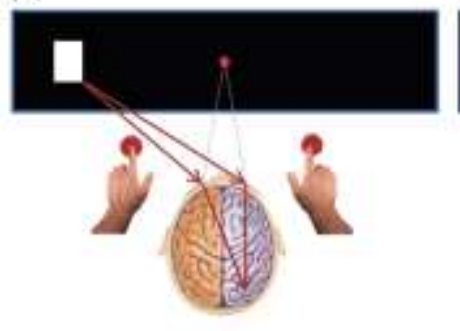

B

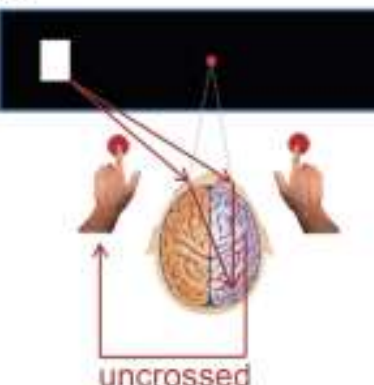

C

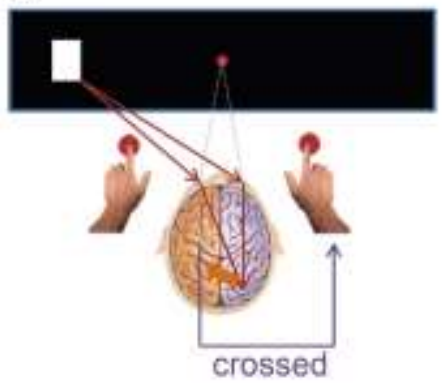

Figure 1. (A) When the visual stimulus is presented in the left visual field, it is first represented in the primary visual cortex of the left hemisphere. (B) Manual responses to this stimulus performed with the left hand do not require interhemispheric communication (uncrossed responses). (C) Manual responses to the same stimulus performed with the right hand require interhemispheric transfer of information (crossed responses) and, therefore, are supposed to be slower.

Roger Sperry's studies on split-brain patients in the 1960s (e.g. Sperry, 1961) increased the scientific interest in lateralization of brain functions and interhemispheric communication. More recently, a meta-analysis of several studies showed that the average CUD in reaction time (RT) was about $4 \mathrm{~ms}$ for more than 300 normal subjects; about $15 \mathrm{~ms}$ for callosal agenesis patients; and from 30 to $60 \mathrm{~ms}$ for split-brain patients (Marzi et al., 1991), revealing the role of the corpus callosum (the biggest brain commissure) in the transfer of visuomotor information.

Although studies with callosal agenesis or split-brain patients directly implicated the corpus callosum in the interhemispheric transfer of visuomotor information, different studies (e.g. Saron et al., 2003; Zaidel and Iacoboni, 2003) claimed that CUD is not resulting from the contralateral organization of the brain, but reflects stimulusresponse spatial compatibility (SRSC) effects (i.e. faster responses to stimuli that are closer to the response effector; Wallace, 1971). Poffenberger himself considered SRSC as a possible confound effect saying that "it is customary to react on the side from which the stimulus comes" (Poffenberger, 1912, p. 70). He rejected this possibility by showing that the spatial compatibility decreased when he presented the peripheral stimulus farther away from the gaze fixation. 
More recently, experiments designed specifically to test if SRSC effects influence RT in the simple Poffenberger paradigm showed that CUD is indeed resulting from anatomical differences between crossed and uncrossed responses (Anzolla et al., 1977; Pellicano et al., 2013). Pellicano et al. (2013), for example, tested if subjects performing bi-manual responses to lateralized stimuli presented slower reaction times for the anatomically crossed hand compared to the anatomically uncrossed hand. The authors tested CUD for hands positioned in the normal hand position (i.e. spatially compatible responses congruent to anatomically uncrossed responses) and for hands positioned in an inverted (one arm laying over the other one as in $\times$ symbol) position (i.e. spatially compatible responses congruent to anatomically crossed responses). Additionally, subjects were separated in two groups: left-handers and right-handers. Pellicano and colleagues (2013) showed that RTs were faster for anatomically uncrossed hands independently on their spatial localization (far or close to the visual stimulus). Moreover, they showed that the CUD is longer when the stimulus is presented in the left visual field (as found in Marzi, 2010) and that this asymmetry tended to be reversed in left-handers (as found in Marzi et al., 1991).

It has been argued that SRSC effects are typically not expected in simple reaction tasks, but in choice tasks that require a flexible, online selection of a correct response (Kornblum et al., 1990; Pellicano et al. 2013). An example of a simple choice task is to select and use the hand ipsilateral to the stimulus (compatible mapping condition) during a block of trials with randomly lateralized stimuli (one stimulus per trial); and the hand contralateral to the stimulus (incompatible mapping condition) in the subsequent block of trials. According to De Jong et al. (1994), ipsilateral (i.e. same side, compatible) responses are automatically activated regardless of whether the required response is compatible or incompatible. This automatic activation must be inhibited during incompatible responses to avoid conflict, creating CUD-like effects.

In the present study, we tested how CUD is modified when we varied subjects' certainty about the upcoming response requirements. Subjects performed three tasks with increasing unpredictability: (a) one task similar to the classic Poffenberger paradigm (i.e. blocks of trials in which the stimulus were presented in the same visual field presentation and the same hand was used to respond); (b) a second task in which the visual field presentation was blocked but the hand use was randomized (with 
correct response cued by the stimulus color); and (c) a third and most unpredictable task in which visual field presentation and hand use were both randomly interleaved.

The unpredictability about which hand subjects should use in the upcoming response period obligated them to select the proper response after the "go cue" (i.e. peripheral visual stimulus). Thus, the second and third tasks were simple action selection tasks and, because of that, subjects might have been under the influence of SRSC effects. The first task, on the contrary, did not require speeded action selection because subjects could prepare the proper manual response before the "go cue", in advance of actual motor response. We hypothesized that CUD and SRSC effects, as well as the amount of (potentially inhibitory) information that should be exchanged between the hemispheres (De Jong et al., 1994), should increase with task uncertainty

In order to locate the areas responsible for transferring the information across the hemispheres and associate their function with the quality (i.e. the character) of the transferred information (e.g. visual, somatosensory, motor), we used event-related fMRI to map areas that are more or less active during crossed as compared to uncrossed responses. Previous studies located putative CUD effects in different brain areas (Table 1).

\section{Table 1}

Areas from the right (R) and left (L) hemispheres related to crossed uncrossed differences in five studies. "-" indicates areas more active during uncrossed responses. Otherwise, areas were more active during crossed responses

\begin{tabular}{|c|c|c|c|c|c|}
\hline Areas & $\begin{array}{c}\text { Marzi } \\
\text { et al. (1999) }\end{array}$ & $\begin{array}{l}\text { Tettamanti } \\
\text { et al. (2002) }\end{array}$ & $\begin{array}{l}\text { Iacoboni } \\
\text { et al. (2004) }\end{array}$ & $\begin{array}{c}\text { Weber } \\
\text { et al. (2005) }\end{array}$ & $\begin{array}{l}\text { Martuzzi } \\
\text { et al. (2006) }\end{array}$ \\
\hline vPFC & & $\mathrm{R}$ and $\mathrm{L}$ & & $\mathrm{R}$ & \\
\hline dlPFC & & $\mathrm{R}$ and $\mathrm{L}$ & $\mathrm{R}$ & & \\
\hline PMd & & $\mathrm{R}$ and $\mathrm{L}$ & $\mathrm{R}$ and $\mathrm{L}$ & & \\
\hline ACC & & $\mathrm{R}$ and $\mathrm{L}$ & $\mathrm{L}$ & $-\mathrm{R}$ & \\
\hline Insula & & $\mathrm{R}$ and $\mathrm{L}$ & & & \\
\hline $\mathrm{CC}$ & & $\mathrm{R}$ and $\mathrm{L}$ & & $\mathrm{R}$ & \\
\hline BA 5 & & $\mathrm{R}$ and $\mathrm{L}$ & & & \\
\hline PCC & & $\mathrm{R}$ & & & \\
\hline SPL & $\mathrm{L}$ & & $\mathrm{R}$ & & \\
\hline Precuneus & $\mathrm{R}$ & $\mathrm{R}$ & $\mathrm{R}$ & & \\
\hline Cuneus & $\mathrm{R}$ & & & $\mathrm{R}$ & \\
\hline STG & & $\mathrm{L}$ & & & \\
\hline MTG & & $\mathrm{L}$ & & $-\mathrm{L}$ & \\
\hline PCC & & $\mathrm{R}$ & & & \\
\hline $\begin{array}{c}\text { Occipital } \\
\text { lobule }\end{array}$ & & & $\mathrm{R}$ & & $\mathrm{R}$ \\
\hline
\end{tabular}


There was no brain area that was associated with CUD across all the mentioned studies. The involvement of the right precuneus in the medial parietal cortex in putative interhemispheric transfer was the most consistent finding (three of the five studies). CUD in reaction times also varied across the studies (from non-significant $1.7 \mathrm{~ms}$ to significant $7 \mathrm{~ms}$ differences) and, to the contrary of CUD in brain signal, was never negative.

Since negative CUD in brain activation was only found in few areas and only in one of the described studies, we expected slower (but more difficult, in a sense) crossed responses to be associated with increased activity, as compared to uncrossed responses. Moreover, we hypothesized that the more predictable is the upcoming response, the earlier (i.e. more visual) stages of the interhemispheric transfer will be manifested. Task comparisons can, therefore, help to elucidate how action selection and certainty modify the intra- and interhemispheric processing and transferring of information.

\subsection{Interspecies differences}

Although the Poffenberger paradigm was extensively tested in humans, we are not aware of any Poffenberger-related study with non-human primates. Interhemispheric communication is essential for goal-directed behavior, being involved in most brain processes. Since monkeys are used as best available animal models in order to translate the knowledge from invasive electrophysiological studies to the understanding of the human brain (Goense et al., 2010), it is very important to test how the interhemispheric transfer of relevant visuomotor information might differ between the two species. Therefore, two rhesus monkeys (Macaca mulatta) also performed the three tasks inside an MRI scanner while we acquired functional images of their brains.

Humans have a behavioral repertoire that greatly exceeds other primates in complexity. Our behavioral complexity was associated with hemispheric specialization (Semendeferi et al., 2002; Wey et al., 2013), which might be a consequence of the enlargement of human neocortex. Since the time associated with interhemispheric communication also increases with neocortex enlargement, this 
expansion imposes constrains to interhemispheric synchronization, favoring presumably more efficient intrahemispheric processing (Aboitiz et al., 2003). In this context, intrahemispheric networks might have been selected by the same evolutionary processes which led to the big human brain (Aboitiz et al., 2003).

But is hemispheric specialization exclusive feature of humans?

The first studies about hemispheric specialization are dated from 1861 with the French anatomist Paul Broca - who associated speech impairments with lesions in a region of the left inferior frontal gyrus (Broca's area) - or even before with Marc Dax in 1836 (Manning and Thomas-Antérion, 2011). Since human language is uniquely complex, the demonstration that aspects of our speech is hemisphere-specific led to the conclusion that brain asymmetry might also be exclusive of humans. Nonetheless, recent studies have been suggested that brain and behavioral asymmetry is more widespread across the animal kingdom than previously assumed, being present in other mammals (e.g. Corballis, 2009), birds (e.g. Güntürkün et al., 2000), reptiles (e.g. Csermelyetal, 2011), amphibians (e.g. Vallortigara, 2006), bony fishes (e.g. Dadda et al., 2010), cartilaginous and jawless fishes (e.g. Concha and Wilson, 2001) as well as invertebrates as Octopus vulgaris (Byrne et al., 2002) and honey bee Apis mellifera (e.g. Frasnelli et al., 2010). The ubiquity of such asymmetry indicates that brain lateralization might constitute a basic principle of the nervous system (Jason et al., 1984; Ocklenburg and Güntürkün, 2012).

Whereas the occurrence of asymmetrical brain/behavior in several species indicates that we should expect hemispheric specializations also for monkeys, there are still reported differences in lateralization gradation between monkeys and humans. The macaque brain is thought to be mostly symmetrical (both anatomically and functionally), and lesions of left or right hemispheres cause comparable contralateral deficits (Gaffan and Hornak, 1997). Oleksiak et al. (2010) provide a review about the main findings (until the publication date) on the lateralization of spatial functioning in primates. The authors conclude that, as expected, spatial lateralization is more prominent in the human brain, possibly as a by-product of the language specialization in the left hemisphere. They emphasize the importance of fMRI studies for direct comparisons between humans and monkeys. In particular, they highlight findings from Baker et al. (2006), which demonstrated clear unilateral saccade-related activations of the (right) pulvinar in two monkeys during a task that involved attention 
and visuospatial orientation. Since this asymmetry was also reported for humans (Fischer and Whitney, 2009), the authors suggested that human distinct organizational principles are more related to cortical regions. In order to support this proposition, Oleksiak et al. (2010) described Kagan et al. (2010) fMRI results, which showed stronger contralateral tuning in the dorsolateral prefrontal cortex, FEF and LIP (all cortical areas) of monkeys performing memory-saccades compared to humans performing the same task. Moreover, the patterns of contralateral selectivity in humans suggested that the left hemisphere encodes predominantly the right space, while the right hemisphere ("free" of most language related processes) represents both visual fields (Kagan et al., 2010; Mesulam, 1999). The longer CUD when the visual stimulus is first represented in the left hemisphere (Marzi, 2010; Pellicano et al., 2013) agrees with this asymmetry because the right hemisphere, by representing both visual fields, would require less communication with the left hemisphere to generate crossed motor responses.

It is important to draw a distinction between related, and often confused, contralaterality and lateralization concepts. For example, the contralaterality of visual processing implied that each hemisphere is responsible for one hemifield, thus, both hemispheres should be involved in the visual processing, and no lateralization (i.e. shift of the entire visual function to one hemisphere) should take place in respect to this function. On the other hand, if the right hemisphere would be able to process both visual fields (as it might be doing starting from the level of the parietal cortex), then the lateralization (i.e. right hemisphere dominance for spatial processing) would be able to emerge. Therefore, contralaterality and lateralization of a specific function are diametrical phenomena (Kagan et al., 2010). In this framework, monkeys are thought to be more contralateral but less lateralized, while humans are less contralateral (besides the primary sensory or motor areas), and more lateralized (i.e. asymmetrical).

Whereas we presume differences in hemispheric asymmetry between species, the overall (non-hemispheric-specific) duration of CUD in reaction time is expected to be shorter in monkeys due to their smaller brain. For example, the interhemispheric distance between V1 in the monkey brain is about 45-47 mm (Innocenti et al. 1995), while in humans axons reach $130 \mathrm{~mm}$ or more to connect the two hemispheres (Aboitiz et al., 2003). Moreover, antidromic transmissions take from 2.6 to $18 \mathrm{~ms}$ in monkeys, while visually evoked potentials have a latency of 13 to $26 \mathrm{~ms}$ in humans 
(Aboitiz et al., 2003). We must consider, however, that these differences in the transmission time might represent methodological discrepancies. Furthermore, it has been argued that the human hemispheres are even more isolated that in monkeys because the relative size of the corpus callosum (adjusted to the brain size differences) decreased in apes and humans as compared to new and old world monkeys (Rilling and Insel, 1999).

Interhemispheric communication time also depends on the axon-to-myelin ratio of the callousal fibers and their diameter (Caminiti et al., 2009). While callosal projections are made by slow-conducting fibers from higher-order cortical regions (as prefrontal and temporoparietal areas), large-caliber fibers provide faster connection between primary and secondary sensorimotor areas (Aboitiz et al., 2003; Fig. 2).

\section{CORPUS CALLOSUM}

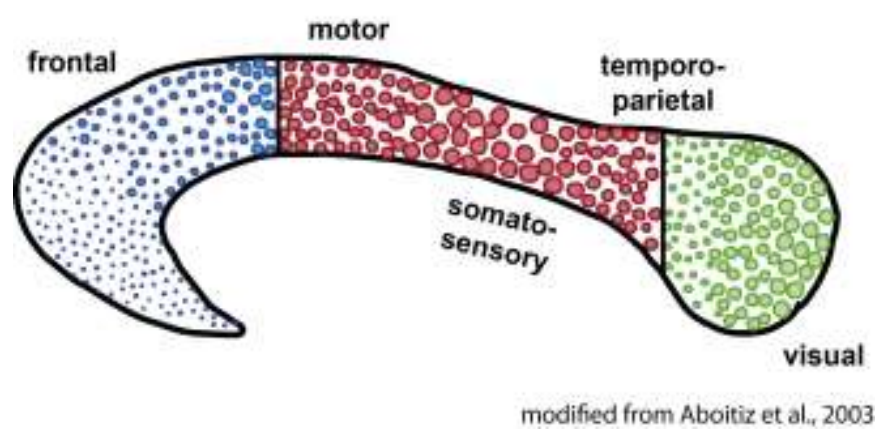

Figure 2. Differences in the diameter of interhemispheric callousal fibers depending on the regions these fibers connect. Fibers from the anterior part of the corpus callosum are mostly thin (slowconducting) and connect areas from the frontal lobules (blue fibers). Fibers from the middle part of the corpus callosum have medium to thick (fast-conducting) diameter and connect motor, somatosensory and temporoparietal areas (red fibers). Lastly, fibers from the posterior part of this commissure have mostly thick diameter and connect visual areas (green fibers). Modified from Aboitiz et al. (2003).

Finally, we also expect interspecies differences in task performance. Although the Poffenberger task is simpler (i.e. low unpredictability about the upcoming response period) than the action selection tasks, all of them require some level of abstract stimulus-response representations.

Contrary to the tasks in which the visual cue is also the target of the movement (e.g. Kagan et al., 2010; Koyama et al., 2004), our experiment requires that subjects represent visual information and associate this representation with movements 
towards a target (button) they are not visualizing. We hypothesize that, since humans are supposed to comprehend better such associations, they still should have better performance than (overtrained) monkeys; especially when responses cannot be prepared in advance but planned and executed under time pressure (action selection tasks).

Whereas previous studies started to provide reliable information about interspecies differences in visuomotor processing (Oleksiak et al., 2010), they also raised a common problem regarding the sample size in studies with monkeys, and difference in methodology. Since the number of individuals in these studies is usually small (2-3 monkeys), individual idiosyncrasies and a history of training might undermine generalizations for the species. In the same time, the individual monkey datasets are typically more robust than in the human studies, because the data is collected repeatedly and are typically analyzed for reproducibility across sessions, while the data collection in humans relies on collecting one or few sessions in each participant and instead derives (potentially different type of) statistical power from averaging across many subjects and identifying only the consistent power. Taking this in the consideration, we compared the results between humans and rhesus monkeys with caution, initially focusing on most clearly identifiable effects.

In summary, in the present study we address the interhemispheric processing of visuomotor information related to flexible responses under the following assumptions: (a) Subjects' uncertainty about the upcoming response requirements modify the interhemispheric communication because it modulates action selection and SRSC effects (e.g. Pellicano et al. 2013); (b) Hemispheric specialization is present in both monkeys and humans but in different degrees. Humans should present stronger lateralization (e.g. Oleksiak et al., 2010); (c) Humans should have more efficient representations of the task requirements; and finally (d) CUD in reaction times and brain signal are influenced by interspecies differences in brain size (Aboitiz et al., 2003).

In order to organize this chapter and convey our messages efficiently, Methods (sections 2 and 4) and Results (sections 3 and 5) are presented separately for humans and monkeys. Afterwards, some of the behavioral results are compared between the two species directly (section 6). 


\section{Humans}

\section{Methods}

\subsection{Subjects}

Fourteen healthy subjects ( 6 males; mean age $25.9 \pm 3.1$ years) were recruited via the student online platform of the University of Goettingen. All subjects had normal or corrected-to-normal vision and responded the Edinburgh questionnaire for handedness assessment (Veale, 2014). They were compensated with $8 €$ per each of the three sessions and provided written consent for participation. The experimental procedures were approved by the local ethics committee.

\subsection{Experimental setup}

Subjects lied inside the MRI scanner in the supine position. The stimuli were backprojected on a translucent screen ( $800 \times 600$ resolution) and viewed by the subjects through a surface mirror mounted on the head coil at approximately $12 \mathrm{~cm}$ from subjects' eyes and $81 \mathrm{~cm}$ from the screen. Subjects maintained central fixation throughout the trial. Gaze position was acquired with $60 \mathrm{~Hz}$ MR-compatible infrared eye tracker camera and ViewPoint 2.8.6.21 software (Arrington Research). Subjects responded with their right and left thumbs using an MR-compatible button box with 4 buttons (Current Designs). The task was controlled via MATLAB (Mathworks) using the Psychophysics toolbox (http://psychtoolbox.org/).

\subsection{Procedure and stimuli}

Subjects performed finger responses under time pressure in response to lateralized visual cues. Eye and hand movements were controlled throughout the trial. Each trial started with the appearance of a red spot and a gray framed-square in the center of the screen. Subjects were correctly positioned in the "rest position" when they fixated the gaze inside the eye fixation window ( $3^{\circ}$ radius of visual angle around the red spot) and, concomitantly, positioned the left and right thumbs over the buttons 1 and 4, respectively. After a varying period, one colored square was presented in the periphery of the screen $\left(13^{\circ}\right.$ of visual angle from its center). The green square cued subjects to respond with the right thumb, while the blue square cued the response with 
the left thumb. Subjects should respond as soon as they saw the cue. The response should be performed in three steps: releasing the right or the left thumb from the respective rest button, pressing the proper target button and coming back to the resting position (Fig. 3B). After that, subjects should hold the rest position for $8 \mathrm{~s}$ until the "success" message appeared on the screen followed by the intertrial interval (Fig. 3A).

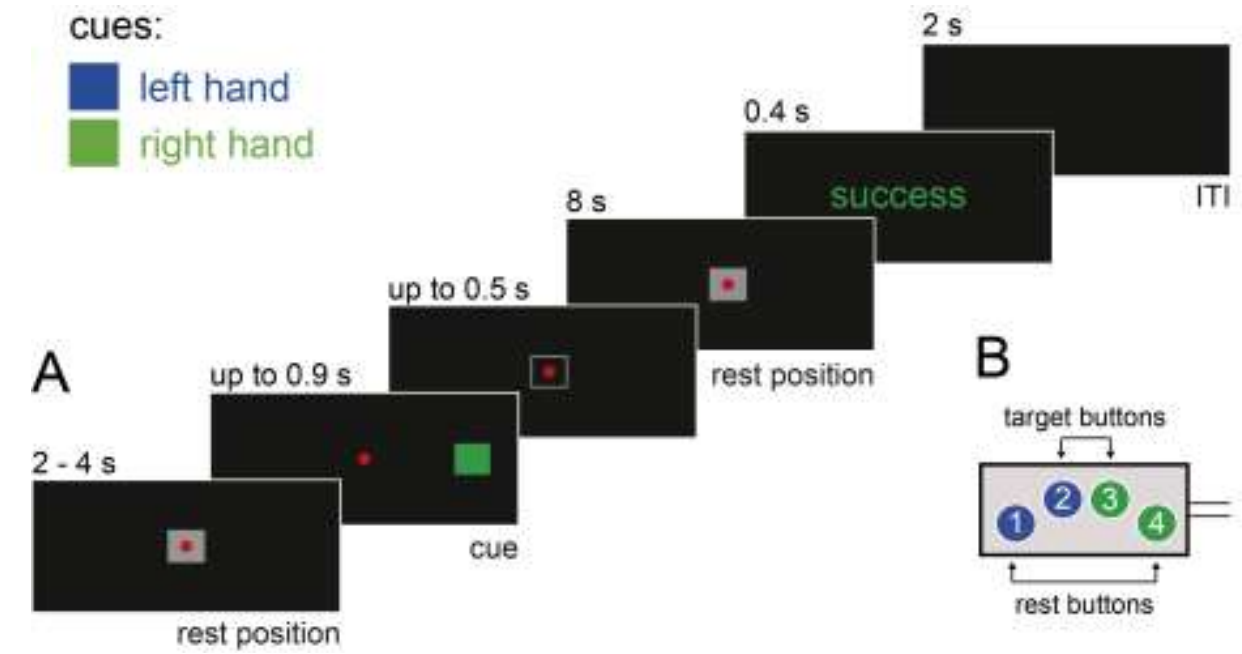

Figure 3. A bright red spot and a gray filled square at the center of the screen indicated that subjects were positioned in the rest position (gaze fixation inside the fixation window and left and right thumbs on the buttons 1 and 4, respectively). After staying in the rest position for a varying period ( 2 to $4 \mathrm{~s}$ ) a colored square was presented at $13^{\circ}$ of visual angle to the right or to the left of the fixation spot, in the same horizontal plane. Subjects had up to $0.9 \mathrm{~s}$ to release the proper resting button ( 1 for the blue cue and 4 for the green cue), $0.5 \mathrm{~s}$ to press the proper target button ( 2 for the blue cue and 3 for the green cue) and $0.5 \mathrm{~s}$ to come back to the resting position. Subjects kept the rest position for $8 \mathrm{~s}$ after the response. Next, the "success" message appeared on the screen for $0.4 \mathrm{~s}$, followed by the intertrial interval (ITI, $2 \mathrm{~s}$ ).

\subsection{Experimental sessions}

Subjects performed three scanning sessions. Each of them contained a different task and included one anatomical scan and four functional runs of 10 minutes. The differences across the sessions (and therefore across the tasks) were the trial sequences and the RT cut-offs. In the No Action Selection (NAS) task, as in the Poffenberger task, a lateralized stimulus (e.g. blue square) was presented in the same visual field for 10 successful trials. After that, the same stimulus was presented in the opposite visual field for the next 10 successful trials. This 20 successful trials 
sequence was then repeated for the other type of stimulus (e.g. green square; Fig. 4A). Moreover, subjects were pre-cued at the beginning of every trial (first rest period) about the hand they should use. This was done by a frame around the fixation square with the same color of the actual cue. By pre-cueing the subjects, we guaranteed that they could prepare their thumb response in advance without using memory-related processes. Moreover, since subjects could prepare their motor responses, this task had a shorter reaction time cut-off (0.6 s), while the following two tasks had the cut-off at 0.9 s. In the Action Selection Blocked Visual field (ASB) task, the lateralized stimulus was presented in the same visual field for 10 successful trials before changing to the other visual field. The stimulus color (and therefore the hand use) was randomized (Fig. 4B). In the Action Selection Random (ASR) task, both visual field presentation and stimulus color were randomized (Fig. 4C). In the three tasks, the sequences were repeated until the end of the run.

Depending on the trial composition (stimulus color and visual field presentation), the responses did not require (i.e. uncrossed condition) or required (i.e. crossed condition) interhemispheric transfer of information (Fig. 4).

\begin{tabular}{|c|c|c|}
\hline left hand / left visual field & = night hand / right visual field & UNCROSSED \\
\hline
\end{tabular}
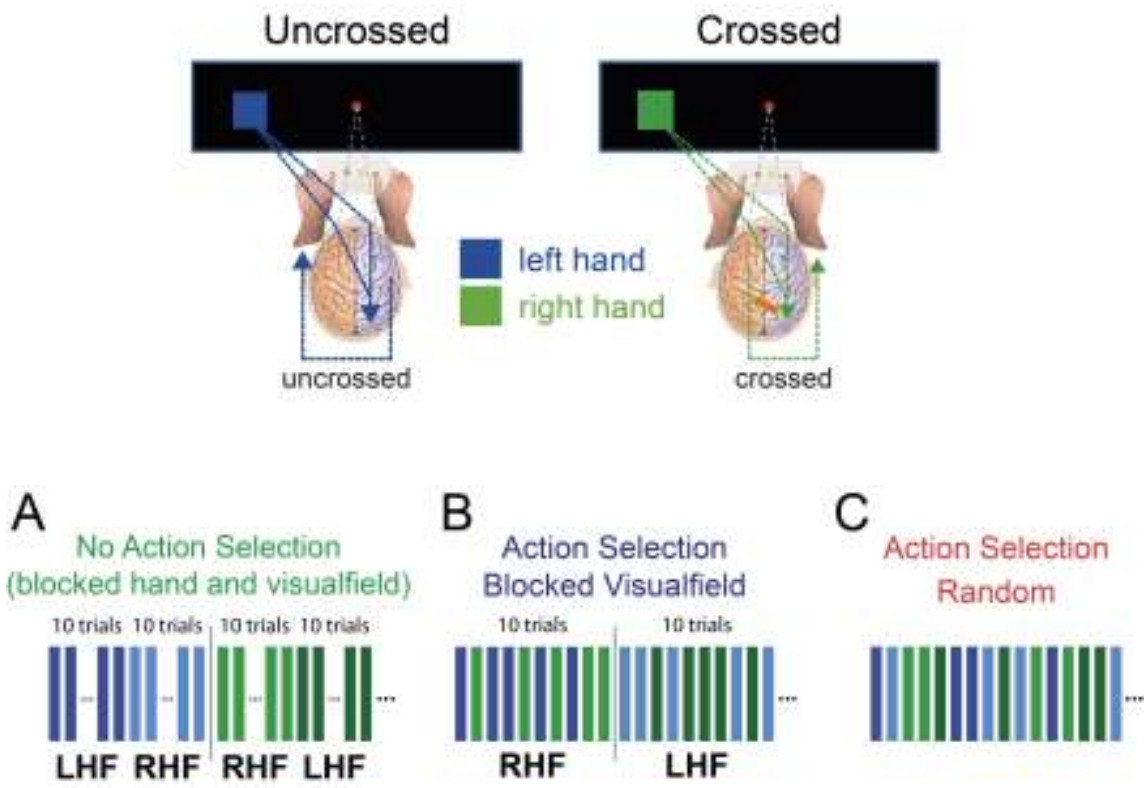

Figure 4. (Top) The combination of stimulus color and visual field presentation determined the trial condition: uncrossed or crossed. (Middle) On the left, an example of uncrossed response (left hand/left 
visual field). On the right, an example of crossed response (right hand/left visual field). The crossed uncrossed difference (CUD) was calculated as the RT difference between averaged crossed and uncrossed responses. (Bottom) Example sequences for each of the tasks: (A) No Action Selection, (B) Action Selection Blocked Visual field and (C) Action Selection Random. Although the structure that defined the task did not change, the starting stimulus color and the starting visual field varied randomly across different runs.

\subsection{MRI Data Acquisition and preprocessing}

Subjects were scanned using a Siemens Trio 3T scanner and a Siemens 32-channel head coil. High-resolution structural scan (T1-weighted MPRAGE volume, $1 \times 1 \times 1$ mm, TR $2300 \mathrm{~ms}$, TE $3.97 \mathrm{~ms}, 192$ slices, flip angle: $9^{\circ}$ ) was acquired for anatomical localization. Functional images were acquired with EPI sequences $(3 \times 3 \times 3 \mathrm{~mm}$, TR $1000 \mathrm{~ms}$, TE $33 \mathrm{~ms}$, 36 transverse slices, multiband-factor 3, $0.15 \mathrm{~mm}$ gap between adjacent slices). The anatomical T1-weighted scans were processed in BrainVoyager QX 2.4 (Brain Innovation). The anatomical images from sessions 2 and 3 were coregistered to the first session in the commissure-posterior commissure (AC-PC) plane and then transformed from AC-PC into Talairach space. Each functional run consisted of 904 volumes. The first four volumes of each run were excluded from functional analyses but were used for coregistration. EPI series from each run were preprocessed in NeuroElf 1.0 (Jochen Weber, www.neuroelf.net) using slice time correction, linear trend removal, and a high-pass temporal filter with three cycles per 10 min run cut-off. The first functional volume in the session was aligned to the original anatomical $\mathrm{T} 1$-weighted scan using rigid body transformations. 3D motion correction with 6 degrees of freedom was done by registering all EPI volumes to the first volume of the first run in the session. 3D volume time courses were computed in Talairach space using the anatomical AC-PC and Talairach transformation parameters (voxel size $3 \times 3 \times 3 \mathrm{~mm})$. Additional spatial smoothing $(6 \times 6 \times 6 \mathrm{~mm})$ was applied to the $3 \mathrm{D}$ volume time courses.

\subsection{Behavioral data analysis}

The behavioral data was analyzed using MATLAB (Mathworks) for performance and RT. RTs reflected the time between the presentation of the cue and the release of the proper rest button. The time interval for transferring information across the hemispheres (Crossed Uncrossed Difference, CUD) was calculated by subtracting the 
average of uncrossed reaction times from the average crossed reaction times (Poffenberger, 1912). We also compared RTs between the hands, using the four combinations of hand-related stimulus (first letter) and visual field presentation (second letter): left hand/left visual field (LL), left hand/right visual field (LR), right hand/right visual field (RR), right hand/left visual field (RL).

\section{7 fMRI data analysis}

One GLM was used to estimate the BOLD response associated with relevant task events. The GLM was performed only for the voxels encompassed in a mask based on the Colin brain (Holmes et al. 1998), transformed into the Talairach space. The regressors were convolved with a canonical hemodynamic response function (time-topositive-peak: $5 \mathrm{~s}$, time-to-negative-peak: $15 \mathrm{~s}$ ).

We used the four combinations of hand-related stimulus and visual field presentation as regressors to model the response event: LL, LR, RR and RL. The main regressors consisted of a boxcar function with onsets at the stimulus presentation and duration of mean RT for the task, convolved with the HRF. These regressors were named differently for each of the three tasks, totalizing twelve main regressors in the GLM that included all the tasks. In addition to the main regressors, the GLM included twelve regressors of no interest: (1-4) wrong use of the hand for each of the four combinations of stimulus color and visual field presentation, (5) trial abortion related to gaze fixation break, (6) success message presentation, and (7-12) six head motion regressors extracted during fMRI data preprocessing.

The relationship between the different modeled events and brain activity was analyzed for each subject. Estimates were tested using contrasts to assess group level significance in two maps with different thresholds: higher threshold map $(\mathrm{p}<0.001$ uncorrected for CUD, spatial and hand tuning) and lower threshold map ( $\mathrm{p}<0.01$ uncorrected for CUD and $\mathrm{p}<0.05$ for spatial and hand tuning). Since we did not find areas more active during uncrossed responses compared to crossed responses, the CUD maps resulted from one-tailed t-tests for deviations in the positive direction. All reported brain activity was corrected for multiple comparisons with family-wise error (FWE, p<0.05) using alphasim of NeuroElf (1000 interactions, $6 \mathrm{~mm}$ smoothing kernel). 


\section{Results}

Fourteen subjects performed speeded manual responses to lateralized visual cues while they kept fixating their gaze in the center of a screen inside the MRI scanner. These subjects completed one of three tasks in different sessions. The tasks varied according to their trial sequence. The less randomized the sequence (NAS $<$ ASB $<$ ASR), the more predictable was the visuomotor contingency in the response period.

\subsection{Behavioral results}

We performed a one-way ANOVA for repeated measures to test if there were differences in reaction times across tasks. Subjects responded faster or slower depending on the task $\left(\mathrm{F}_{2,26}=143.649, \mathrm{p}<0.0001\right)$. Post-hoc tests showed that the reaction times did not differed between action selection tasks (ASB vs ASR; $\mathrm{t}_{13}=0.972, \mathrm{p}>0.05$ ), but were faster in the task that did not require action selection (NAS) compared to the ASB $\left(\mathrm{t}_{13}=13.994, \mathrm{p}<0.0001\right)$ and to the ASR $\left(\mathrm{t}_{13}=13.636\right.$, $\mathrm{p}<0.0001)$ tasks. Figure 5A illustrates the reaction time distribution for all correct trials, even though the tests were performed across subjects' averages.

Next, we performed one paired t-test for each task to test CUD in reaction time. Both action selection tasks presented positive and significant CUD (ASB, $\mathrm{t}_{13}=-3.222$, $\mathrm{p}<0.05$; ASR, $\left.\mathrm{t}_{13}=-6.828, \mathrm{p}<0.001\right)$, but crossed and uncrossed responses took the same time in the NAS task $\left(\mathrm{t}_{13}=-0.284, \mathrm{p}>0.05\right.$; Fig. 5B).

A

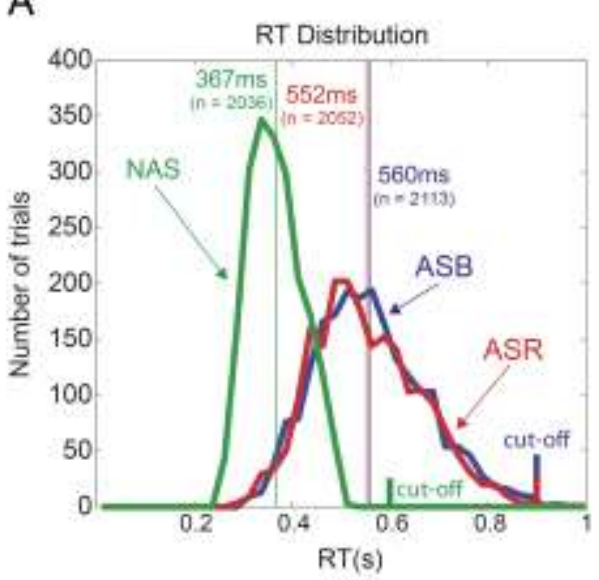

B

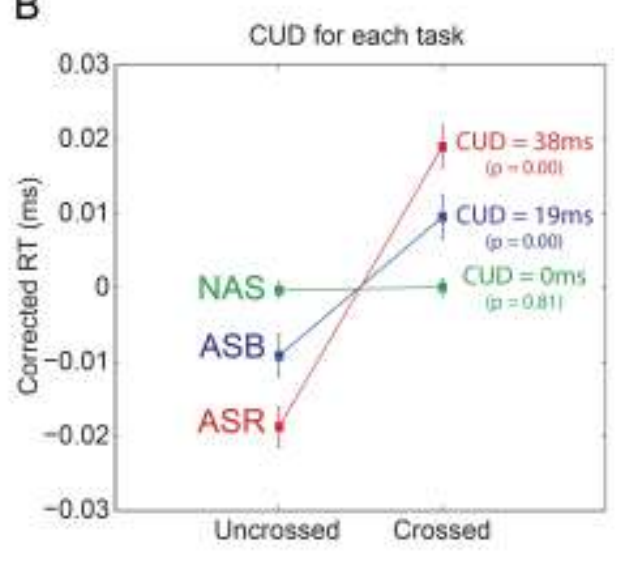


Figure 5. (A) Histogram of the RT distribution of all correct trials for the three tasks, and their respective reaction time cut-offs. (B) Means and standard errors, across subjects, of crossed and uncrossed reaction times for the three tasks, corrected with the average reaction time of the corresponding hand (i.e. reaction times for the LL and LR conditions were corrected with the averaged left hand reaction time). No Actions selection task (NAS): green letters and curves. Action Selection Blocked visual field task (ASB): blue letters and curves. Action Selection Random (ASR): red letters and curves.

We also tested CUD in RT for each hand separately (one paired t-test for each hand). In general, these results were similar to the previous results (Fig. 5B), since no significant difference was found in the NAS task (left hand: $\mathrm{t}_{13}=1.001, \mathrm{p}>0.05$, right hand: $\left.\mathrm{t}_{13}=-1.415, \mathrm{p}>0.05\right)$, while both hands presented significant CUD in the action selection tasks (ASB left hand: $\mathrm{t}_{13}=-2.611, \mathrm{p}<0.02$, right hand: $\mathrm{t}_{13}=-2.261, \mathrm{p}<0.05$; ASR left hand: $\mathrm{t}_{13}=-4.059, \mathrm{p}<0.001$, right hand: $\mathrm{t}_{13}=-8.375, \mathrm{p}<0.001$; Fig. 6). Nevertheless, we found opposite, although not significant, effects between the hands during the NAS task. While the right hand presented positive CUD, crossed responses with the left hand were faster than the uncrossed responses (Fig. 6A).

A

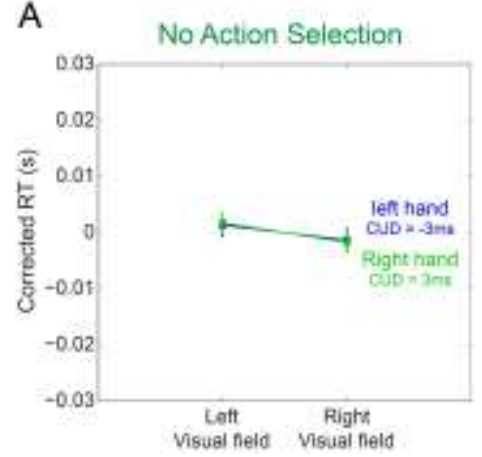

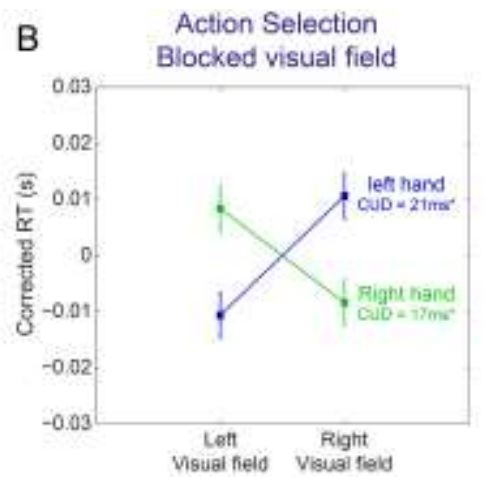

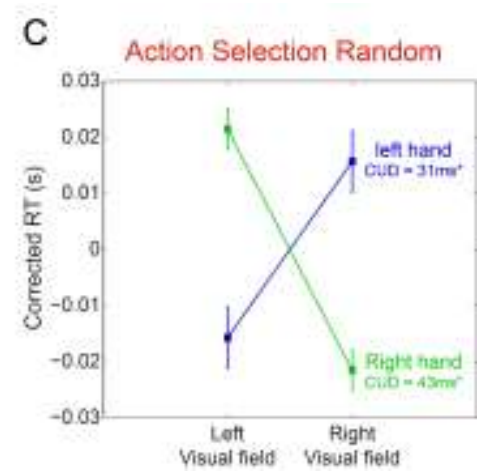

Figure 6. Reaction times for each hand (blue line for the left hand and green line for the right hand) related to each visual field for the (A) NAS, (B) ASB and (C) ASR tasks. *t-test ( $\mathrm{p}<0.05)$.

Although the CUD was significant on average across subjects in the ASB and ASR tasks, not all subjects had individually significant CUD across trials (Fig. 7), suggesting high intersubject variability, especially for the ASB task which had only 5 of the 14 subjects with significant CUD across trials. 


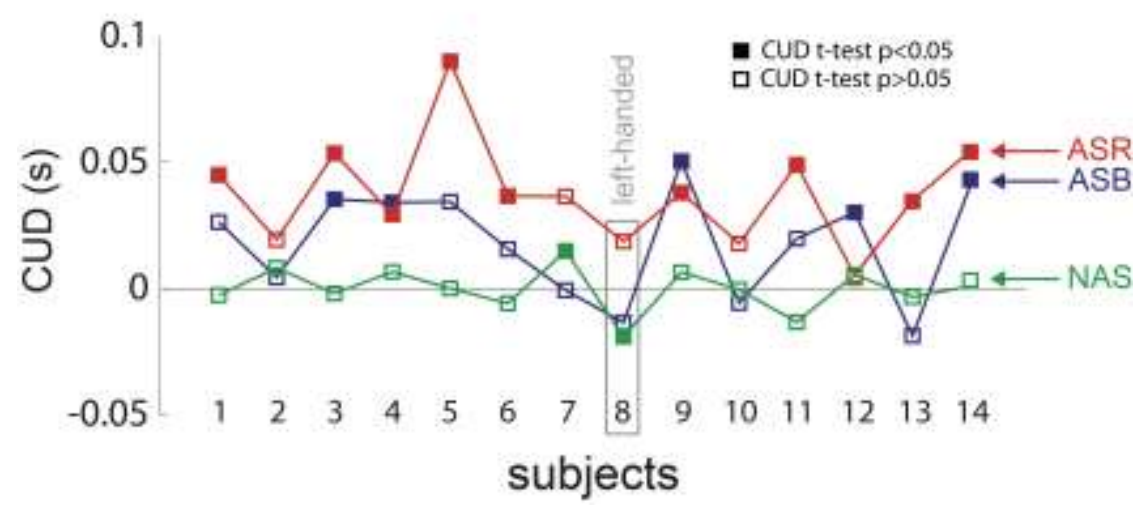

Figure 7. CUD of each subject in each task. Filled squares represent significant CUD (t-test, $\mathrm{p}<0.05)$.

Finally, humans presented the same performance in all tasks (NAS: 93\%, ASB: 95\% and ASR: $\left.94 \% ; \mathrm{F}_{2,26}=1.285, \mathrm{p}>0.05\right)$.

\section{2 fMRI results}

The visual stimulation (i.e. stimulus size and distance from the fixation spot) and the motor response (i.e. finger movements) were similar across trials and tasks. Nevertheless, differences in trial sequence generated different levels of predictability across tasks (NAS>ASB $>$ ASR) about the upcoming response period, impacting action selection requirements and brain activation patterns. In general, effector (left/right hand) and spatial (left/right visual field) contralateral tuning was observed with similar patterns in the statistical activation maps of the three tasks, expect for the spatial tuning in the NAS task (see below). Moreover, as predicted, the activations associated with crossed responses were stronger than on uncrossed responses in all the tasks.

In the No Action Selection (NAS) task, only few areas were associated with differences between crossed and uncrossed responses (crossed uncrossed difference, CUD). Strikingly, there was no apparent contralateral spatial tuning in the left hemisphere that survived a high statistical threshold $(\mathrm{p}<0.001$ uncorrected), while ipsilateral activations were observed in the right inferior frontal gyrus (IFG; Fig. 8). 


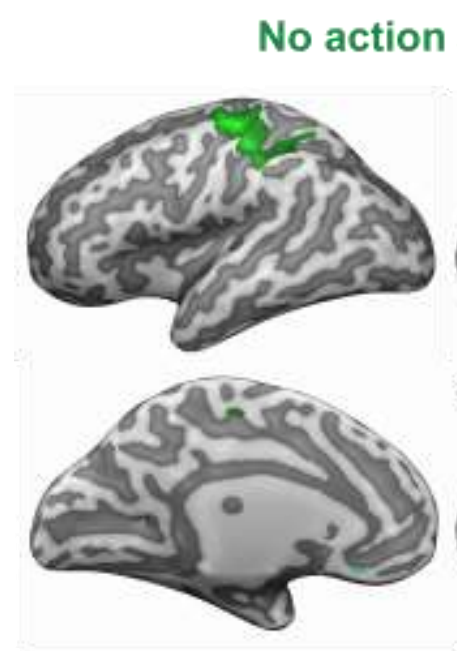

Left Hemisphere

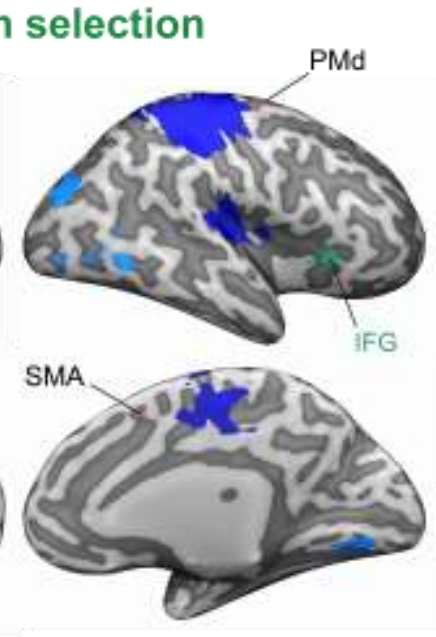

Right Hemisphere

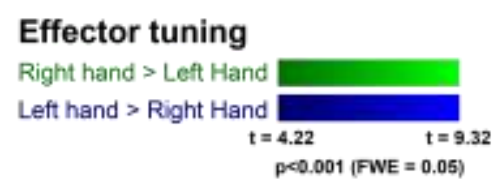

Space tuning (visual field, VF)

Right VF $>$ Left VF

Left WF 2 Right WF

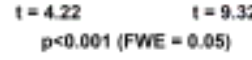

Crossed-uncrossed tuning

CROSSED $=$ UNCROSSED $\quad t=3.85 \quad t=9.32$

Figure 8. High threshold maps on the inflated brain for three activation patterns when subjects performed the NAS task. Areas in dark green and blue reflect effector tuning for right and left hands, respectively. Areas in light green and blue reflect space tuning for right and left visual fields, respectively. The main contrast (orange) mapped areas more active during crossed compared to uncrossed responses. There were no areas more active during uncrossed than during crossed responses.

The ipsilateral visual-related activation in the right hemisphere under this high threshold indicates that the right hemisphere represents both visual fields as suggested by Mesulam (1999) and Kagan et al. (2010). The expected contralateral spatial tuning in early visual areas can be observed with a more permissive threshold (Supplementary Fig. 1). The information about the clusters present in the lower threshold CUD map (which also includes the higher threshold clusters) can be seen in the Supplementary Table 1.

The CUD map of the Action Selection Blocked visual field (ASB) task was the most extensive one. This map was characterized by the activations in the middle frontal gyrus (MFG) - including the dorsolateral prefrontal cortex (dlPFC) - and in the IFG (Fig. 9). 
Action Selection Blocked visual field

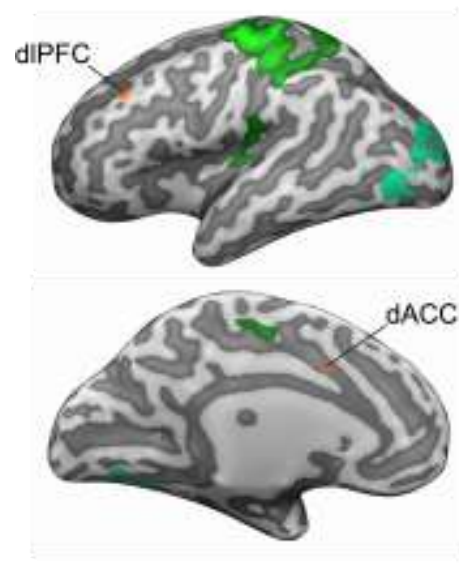

Left Hemisphere

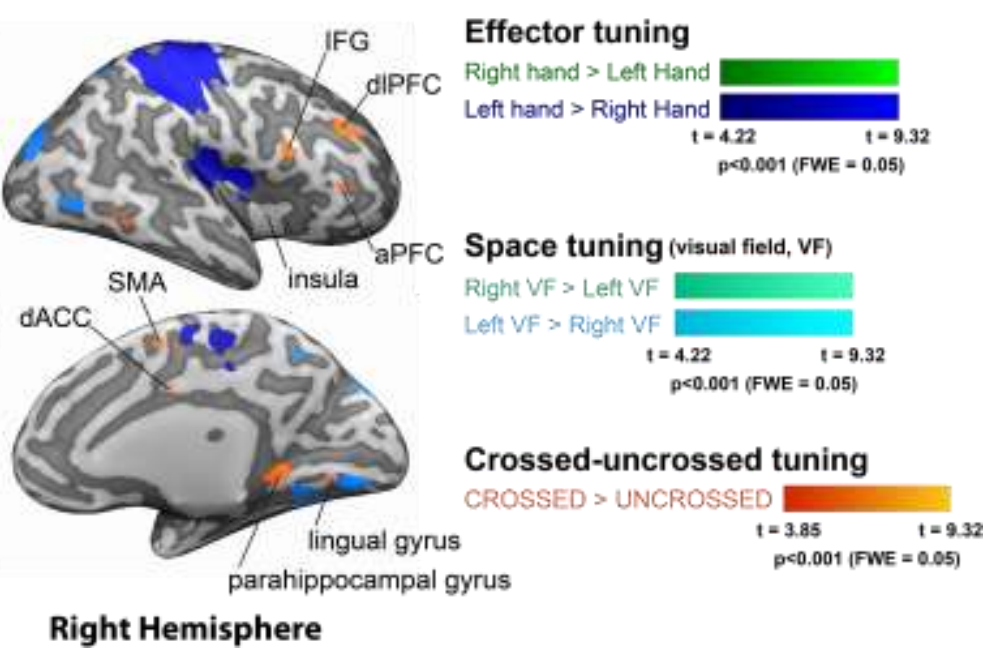

Figure 9. Higher threshold maps on the inflated brain for three activation tunings when subjects performed the ASB task. Areas in dark green and blue reflect effector tuning for right and left hands, respectively. Areas in light green and blue reflect space tuning for right and left visual fields, respectively. The main contrast (orange) mapped areas more active during crossed compared to uncrossed responses. There were no areas more active during uncrossed than during crossed responses.

At the lower threshold, the CUD map of the ASB task was more symmetrical, especially in frontal areas as insula, and included areas from the premotor and the inferior parietal cortices (Supplementary Fig. 2).

Finally, the CUD map of the Action Selection Random (ASR) task contained mainly areas from the parietal and temporal lobules. It is important to note the overlap between the effector tuning and the CUD maps in the parietal lobule (Fig. 10), which suggests that motor-related areas were involved in the transfer of interhemispheric information during this task. 


\section{Action Selection Random}

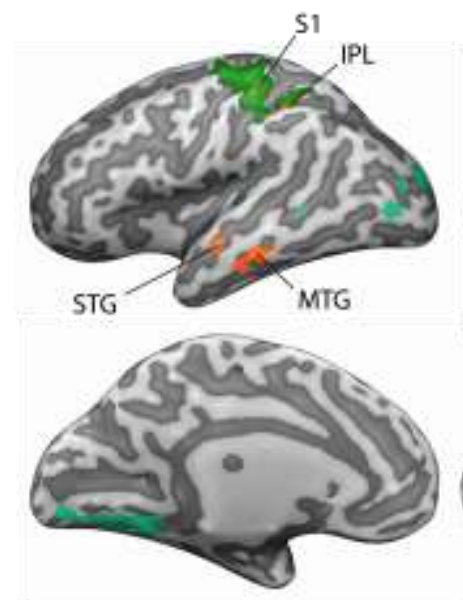

Left Hemisphere
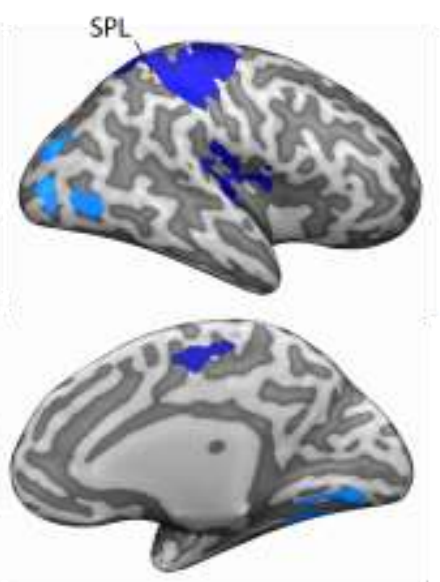
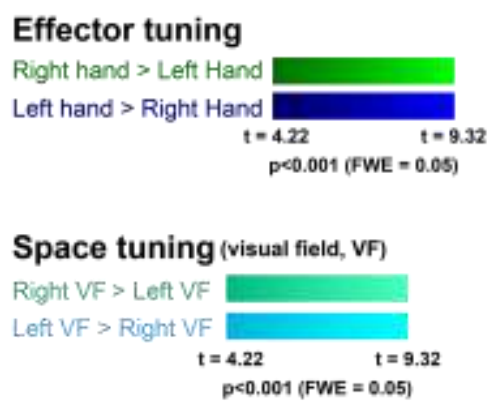

Crossed-uncrossed tuning

CROSSED $>$ UNCROSSED

Figure 10. Maps on the inflated brain for three activation tunings when subjects performed the ASR task. Areas in dark green and blue reflect effector tuning for right and left hands, respectively. Areas in light green and blue reflect space tuning for right and left visual fields, respectively. The main contrast (orange) mapped areas more active during crossed compared to uncrossed responses. There was no area more active during uncrossed than during crossed responses.

The lower threshold CUD map for the ASR task was also more symmetrical, especially for temporal areas (Supplementary Fig. 3). The only CUD area present on all lower threshold maps is the inferior parietal lobule (IPL). There was no overlap between the CUD maps of the three tasks.

Although clusters in the CUD maps presented, on average, higher activations for the crossed (LR and RL) conditions than for the uncrossed (LL and RR) conditions, some of them also demonstrated spatial or hand tuning. In the Figure 11, we exemplify the beta values of three different areas of the right hemisphere for the ASB task. The activations in the right dorsolateral prefrontal cortex (right dlPFC) were most probably driven exclusively by CUD (LR+RL $>$ LL+RR; Fig. 11A). The right cuneus, on the other hand, was tuned to CUD and also presented spatial tuning (RL+LL>LR+RR; Fig. 11B), while the right thalamus presented CUD and higher activation for conditions associated with the contralateral left hand $(L L+L R>R R+R L$; Fig. 11C). 

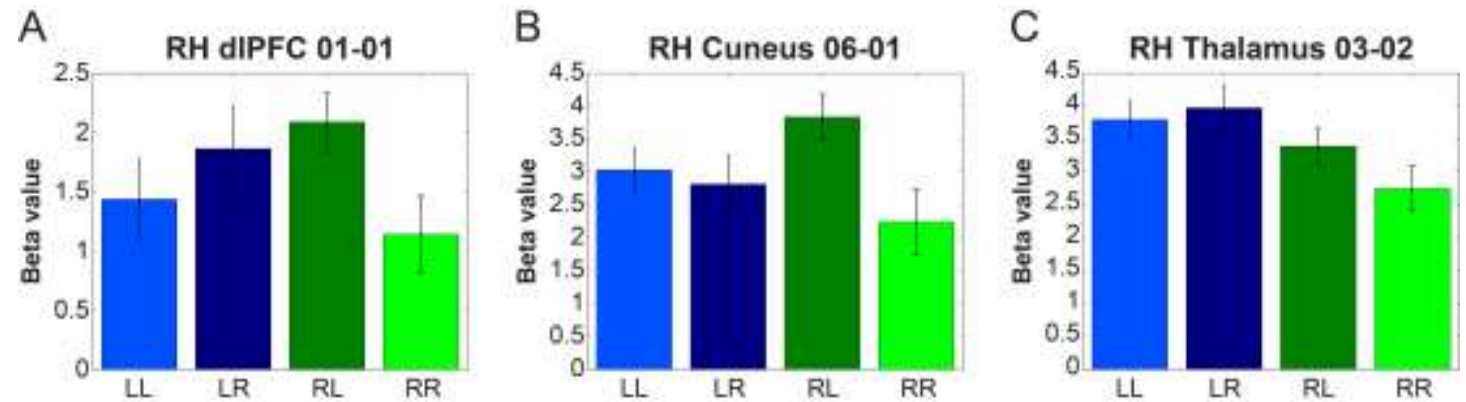

Figure 11. Mean beta values and standard errors of the four task main regressors (LL, LR, RL and RR) for three different areas (dlPFC, cuneus and thalamus) in the right hemisphere (RH) during the ASB task. Together with the CUD-related activity, cuneus also presented spatial tuning (left visual field > right visual field), and thalamus presented hand tuning (left hand > right hand).

Next, we performed two statistical comparisons in order to locate areas involved in action selection. The first one contrasted the two tasks involving action selection with the No Action Selection task (ASB+ASR>NAS). The second one contrasted only ASB with NAS (ASB $>$ NAS). This contrast is more reliable since in both tasks the visual field presentation of the lateralized cue varied in blocks, while differences between ASR and NAS can be related to action selection, but also with the random location of the peripheral visual stimulus in the ASR task. Together with areas from the ventral occipital lobule and with the insula (present in the ASB+ASR >NAS map), areas from the dorsal parietal lobule (as precuneus, superior parietal lobule and somatosensory cortex) and from the cingulate cortex (dACC) were more active when action selection was required (Fig. 12). There was no area more active during the NAS task compared to the other tasks. 


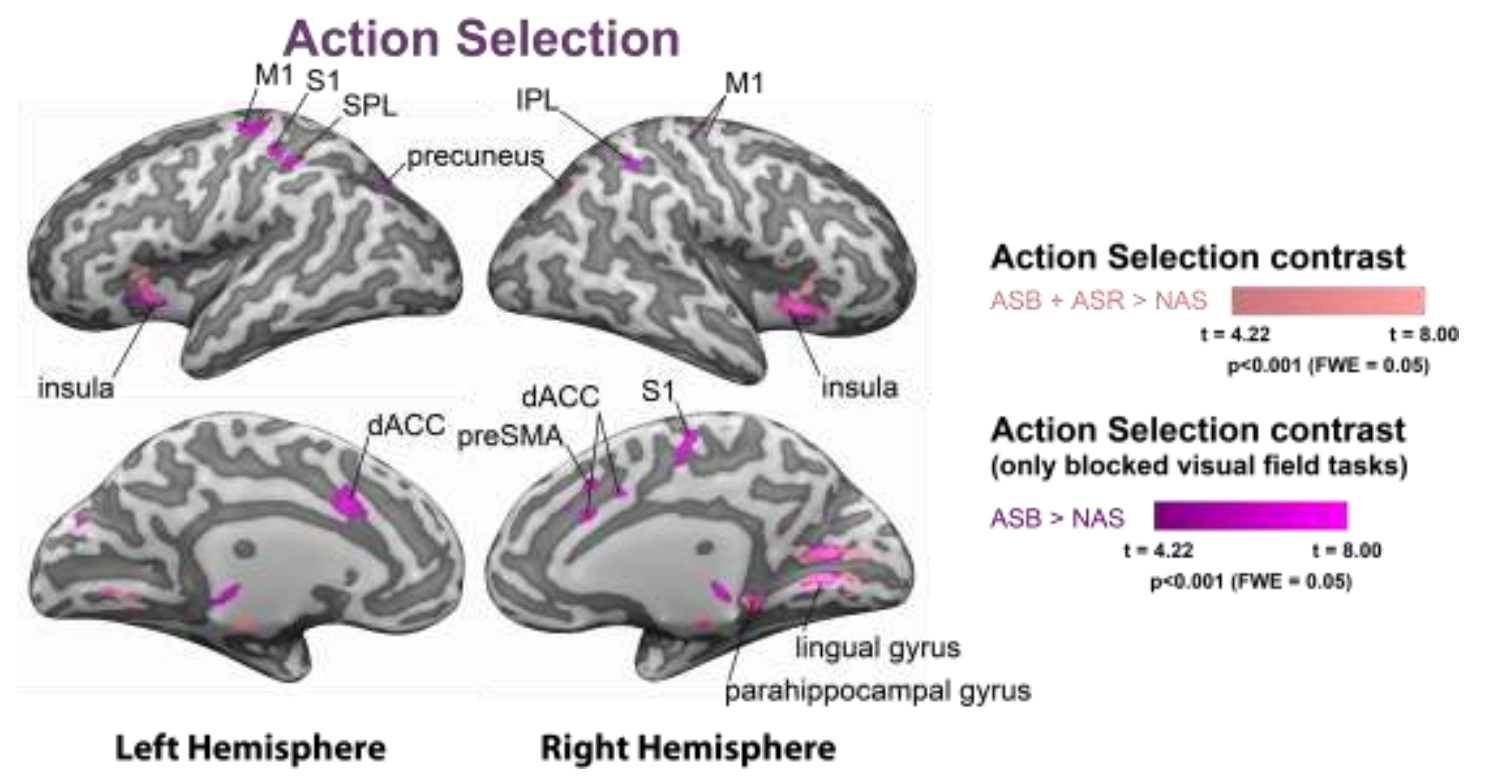

Figure 12. Maps on the inflated brain for two contrasts related to action selection. The first one contrasted both action selections tasks (ASB and ASR) with NAS. The second one only contrasted the tasks in which the location of the visual stimulation varied in blocks of trials (ASB and NAS). There was no cortical area that was more active when action selection was not required.

Most of the described CUD-related activation occurred in the cortex of the brain and, therefore, was represented in the inflated brains (Fig. 8-10). Nevertheless, there were activations in four non-cortical areas which we consider important to report.

In the NAS task, there was an activation cluster in the white matter close to the right cingulate gyrus. This was the only CUD-related activity in the white matter we mapped, and it occurred in the same side of the ipsilateral spatially tuned activation. In the ASB task, the right thalamus was more activated in crossed than in uncrossed responses (Fig. 13).
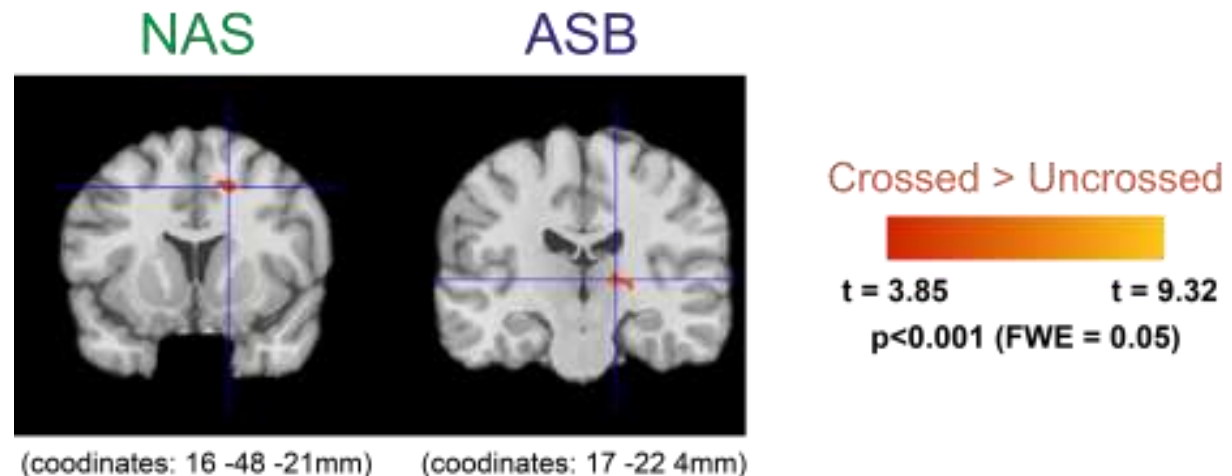

Figure 13. Non-cortical areas presenting higher activity on crossed compared to uncrossed responses. On the left, a cluster in the white matter of the right cingulate gyrus presented CUD in the activity 
during the NAS task. On the right, the right thalamus was more responsive during crossed responses in the ASB task.

We also identified CUD in the activity of cerebellar areas. During the NAS task, the cerebellar CUD activation was more posterior (at the pyramis) than during the ASR task. The cerebellar hand tuning was very similar across the three tasks. In the ASR task, the right dentate presented both: hand tuning and CUD tuning (Fig. 14); repeating the overlapping brain cortical pattern for the task (Fig. 10).
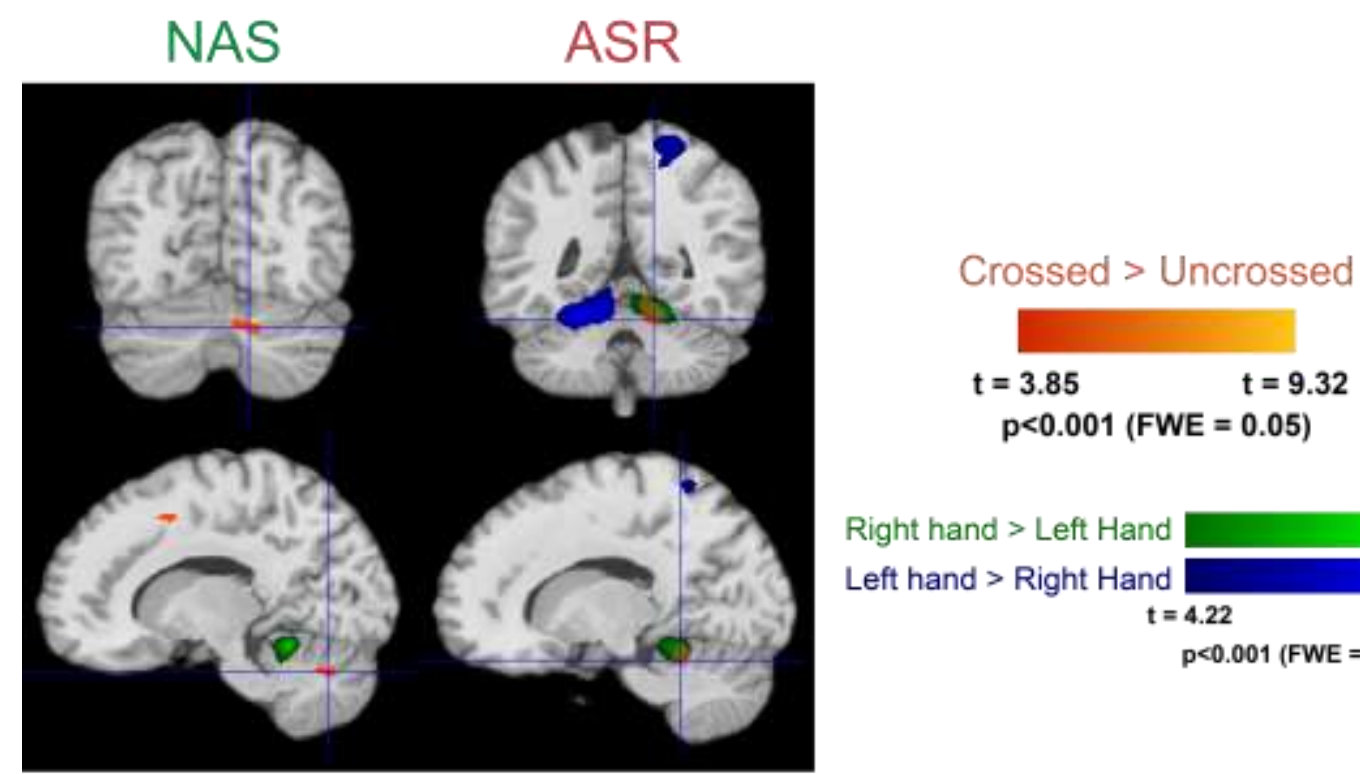

(coodinates: $12-70-29 \mathrm{~mm}$ )

Figure 14. Cerebellar areas presenting higher activity on crossed compared to uncrossed responses. The CUD cerebellar activity in the NAS task, on the left, was more posterior and did not overlap with the hand tuned activity as it did for the ASR task, on the right.

Together with the increased activity in the dorsal parietal, cingulate and inferior occipital cortices described in the Figure 12, action selection was also related to increased activity in thalamic areas compared to the No Action Selection task (Fig. $15)$. 


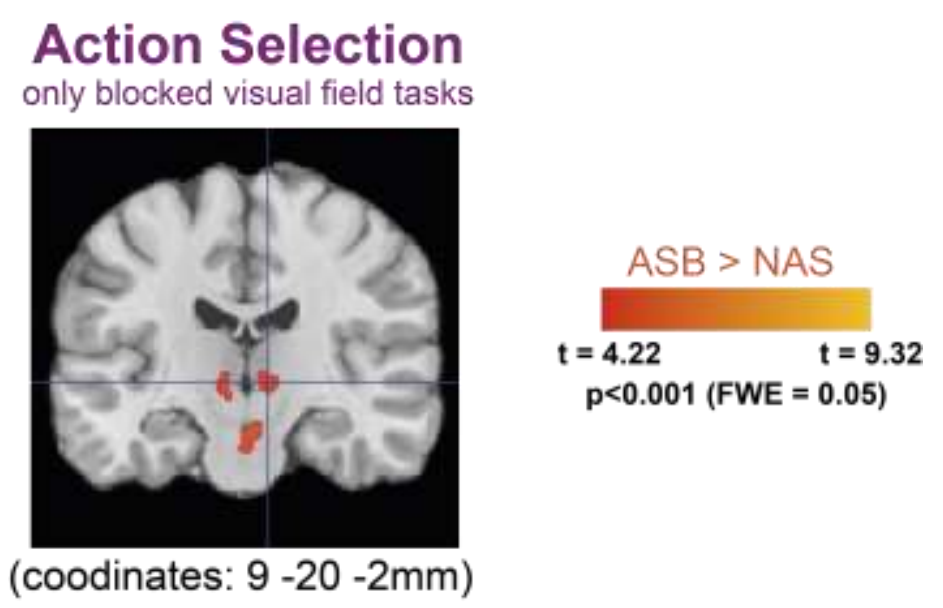

Figure 15. Thalamic clusters in both sides of the brain were more active when action selection was required than in the NAS task.

\section{Rhesus monkeys}

\section{Methods}

\subsection{Subjects}

Two male rhesus macaques (Macaca mulatta) weighting 8-11 kg were implanted with MR compatible plastic (PEEK) headposts embedded in Palacos bone cement (Heraeus). The headposts were attached to the cranium with short ceramic screws while the monkeys were under general anesthesia. Animal care and all experimental procedures were conducted in accordance with German laws governing animal care and approved by local authorities.

\subsection{Experimental setup}

For training and scanning, monkeys sat in a specially designed horizontal MR chair with the head rigidly attached to the chair with a PEEK headholder. Inside the scanner, stimuli were back-projected on a translucent screen at around $64 \mathrm{~cm}$ of monkeys' eyes. The monkeys were positioned inside the MRI scanner in the prone position. They maintained central fixation throughout the trial. Gaze position was monitored with $60 \mathrm{~Hz}$ MR-compatible infrared eye tracker camera and ViewPoint 2.8.6.21 software (Arrington Research). A second MR-compatible camera monitored jaw movements associated with licking the liquid reward, that were detected with a PELCO MD2001 motion detection system. Monkeys responded with their right and 
left hands using four customized MR-compatible buttons (Current Designs) mounted on a plastic support. Lastly, a plate positioned between their head and hands occluded visual information about hands location (Fig 16A). The task was controlled via MATLAB (Mathworks) using the Psychophysics toolbox (http://psychtoolbox.org/).

\subsection{Trial timeline}

Monkeys performed speeded hand responses under time pressure while they kept fixating their gaze in the center of the screen. Eye and hand movements were controlled throughout the trial. Each trial started with the appearance of a red spot and a gray framed-square in the center of the screen. Monkeys were correctly positioned in the "rest position" when they fixated the gaze inside the eye fixation window $\left(3^{\circ}\right.$ radius of visual angle around the red spot) and, concomitantly, positioned the left and right hands over the buttons 1 and 4, respectively (Fig. 16A). After a varying period, one colored cue was presented at the periphery of the visual field $\left(13^{\circ}\right.$ of visual angle from the center of the screen). Squares cued monkeys to release the right (green square) or the left (blue square) hand from the respective rest button, press the proper target button ( 2 for the left hand and 3 for the right hand) and come back to the resting position. Monkeys should respond as soon as they saw the cue. The orange triangle cued monkeys to withhold their hand responses and keep the rest position. After the response (or withhold) period, monkeys should hold the rest position until the juice reward was delivered (Fig. 16B).

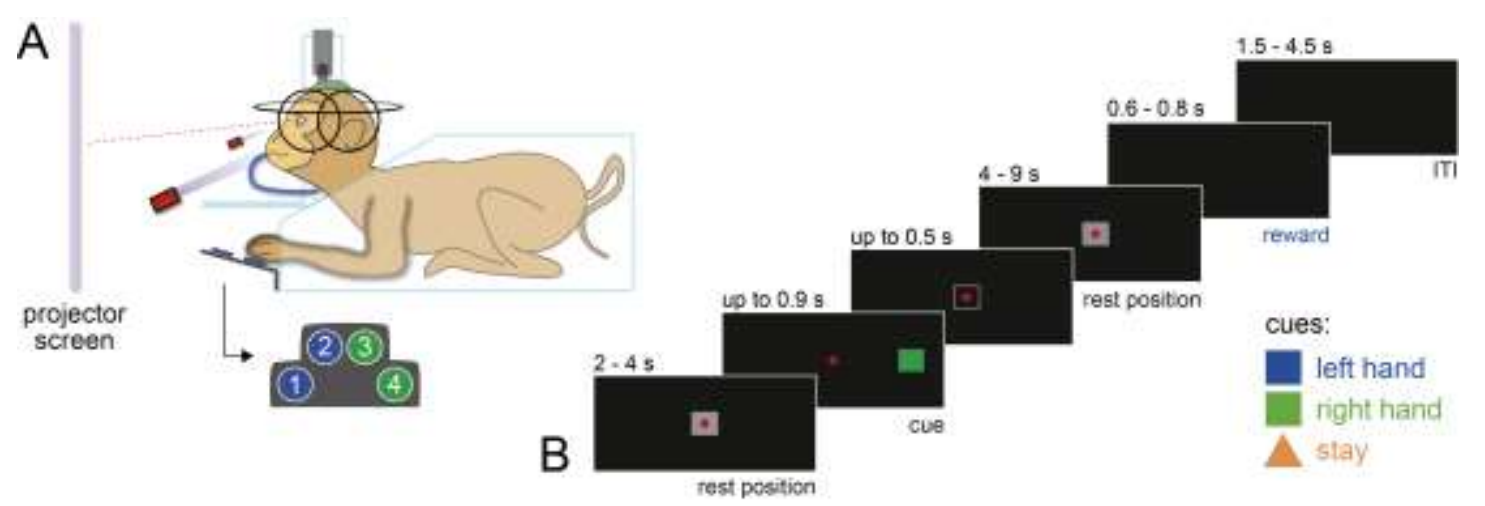

Figure 16. (A) Stimuli were back-projected on a screen inside the MRI scanner while the monkeys sat in the prone position inside a horizontal MR chair. Their head was attached to the chair. We checked gaze fixation and jaw movements with MR-compatible cameras. The monkeys responded with their 
right and left hands using four buttons. Buttons 1 and 4 were used to maintain rest position, while buttons 2 and 3 were used as targets during hand responses. A plate positioned between their head and hands occluded visual information about hands location. (B) A bright red spot and a gray filled square at the center of the screen indicated that monkeys were positioned in the rest position (gaze fixation inside the fixation window and left and right hands over the buttons 1 and 4, respectively). After staying in the rest position for a varying period ( 2 to $4 \mathrm{~s}$ ) a colored square or an orange triangle was presented at $13^{\circ}$ of visual angle to the right or to the left of the fixation spot, in the same horizontal plane. Monkeys had up to $0.9 \mathrm{~s}$ to release the proper resting button ( 1 for the blue square and 4 for the green square), $0.5 \mathrm{~s}$ to press the proper target button ( 2 for the blue square cue and 3 for the green square cue) and $0.5 \mathrm{~s}$ to come back to the resting position. They kept the rest position for a period varying from 4 to $9 \mathrm{~s}$ after the response. Next, the juice reward was delivered, followed by the intertrial interval (ITI, $2 \mathrm{~s})$.

\subsection{Experimental sessions}

Daily experimental sessions lasted from 2 to 4 hours. During this time, monkeys usually completed five to seven functional runs of 15 min each after shimming, adjustment, and anatomical scans. Altogether, monkeys completed 49 sessions (24 for monkey B and 25 for monkey C). They performed one task in each session. In the No Action Selection (NAS) task, a square (e.g. blue square) was presented in the same visual field for 10 successful trials. After that, the same stimulus was presented in the opposite visual field for the next 10 successful trials. This 20 successful trials sequence was then repeated for the other square color (e.g. green cue; Fig. 17A), followed by a sequence of orange triangles that was half as long as the squares sequences ( 5 successful trials in each visual field). Monkeys were also pre-cued at the beginning of every trial in order to allow them to prepare or avoid their motor responses in advance without using memory-related processes. In the Action Selection Blocked Visual field (ASB) task, the lateralized stimulus was presented in the same visual field for 10 successful trials before changing to the other visual field. Stimuli (squares and triangle) were randomized (Fig. 17B), except for trials following the use of the wrong hand for the motor response. In those cases, the same stimulus used in the previous trials was presented again. For simplification, we named these trials as "after wrong hand trials". In the Action Selection Random (ASR) task, both visual field presentation and stimuli (squares and triangle) were randomized (Fig. 17C), except after wrong hand trials. The repetition of unsuccessful trials was used to prevent monkeys from stop utilizing one of the hands. For all the three tasks, the 
proportion of green squares, blue squares and orange triangles was around 40, 40 and $20 \%$, respectively; and the sequences were repeated until the end of the run (Fig. 17).

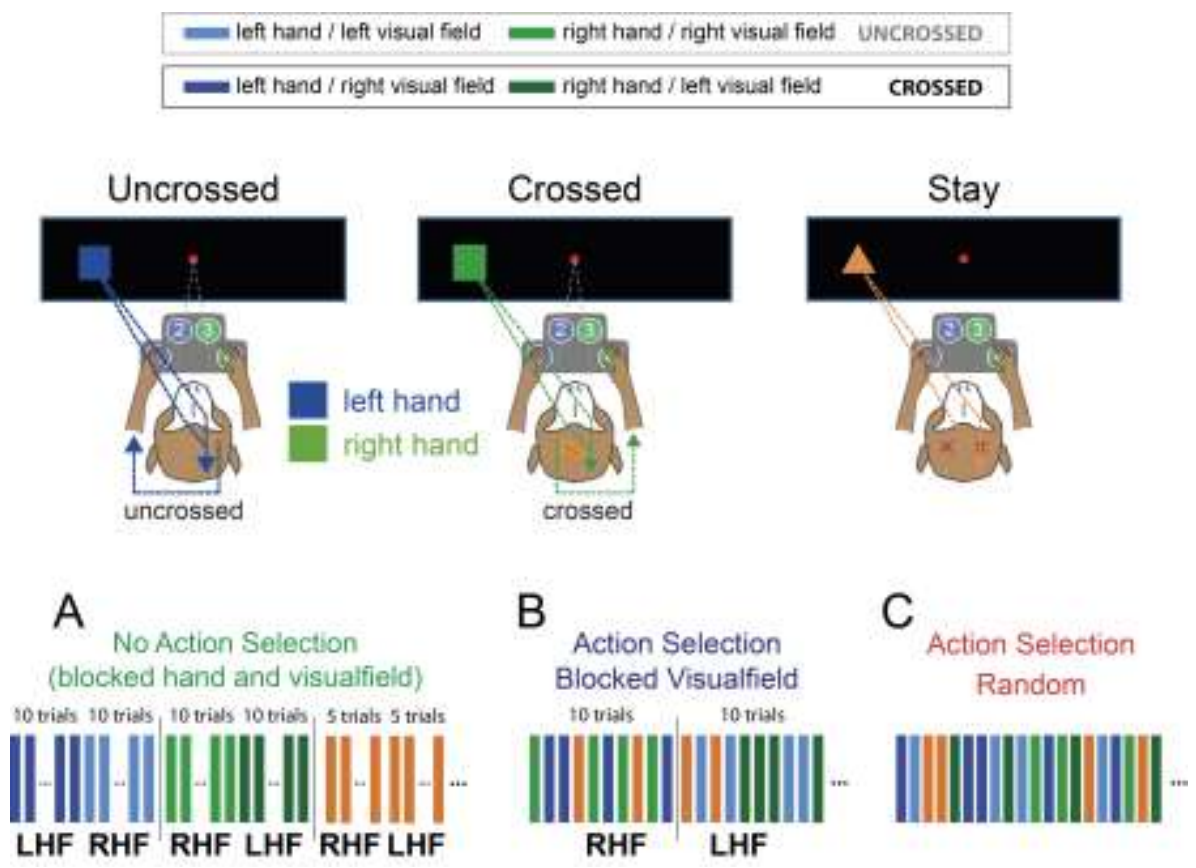

Figure 17. (Top) The four combinations of stimulus color and visual field presentation determined the trial condition: uncrossed or crossed. (Middle) On the left, an example of uncrossed response (left hand/left visual field). On the middle, an example of crossed response (right hand/left visual field). On the right, an example of stay cue withholding the monkey motor response. The crossed uncrossed difference (CUD) was calculated as the reaction times (RT) difference between averaged crossed and uncrossed responses. (Bottom) Example sequences for each of the tasks: (A) No Action Selection, (B) Action Selection Blocked Visual field and (C) Action Selection Random. Although the structure that defined the task did not change, the starting stimulus and the starting visual field varied randomly across different runs.

\subsection{MRI Data Acquisition and preprocessing}

Monkey B was scanned using a Siemens Trio 3T scanner and the Monkey C was scanned using a Siemens Prisma 3T scanner. A linear transmitter-receiver birdcage volume RF coil allowed whole-head homogeneous coverage. The global SNR $\left(\right.$ mean $\left._{\text {signal }} / \mathrm{SD}_{\text {noise }}\right)$ in echo-planar imaging (EPI) was in the range of 36-48 for Monkey B and 52-72 for monkey C.

The whole-head high-resolution (1 mm voxel) T1-weighted image was obtained in a separate session. Functional images were acquired with EPI sequences $(1.2 \times 1.2 \times$ 
$1.2 \mathrm{~mm}$, TR $2000 \mathrm{~ms}$, TE $27 \mathrm{~ms}, 30$ transverse slices for Monkey B and 32 transverse slices for monkey $\mathrm{C}, 76^{\circ}$ flip angle). The anatomical scans were processed in BrainVoyager QX (Brain Innovation). The in-plane anatomical images from all sessions were coregistered to the first session, which was coregistered to high resolution anatomical image in the commissure-posterior commissure (AC-PC) plane. Each functional run consisted of 904 volumes. The first four volumes of each run were excluded from functional analyses. EPI sequences from each run were preprocessed in NeuroElf (www.neuroelf.net) using slice time correction, linear trend removal, and a high-pass temporal filter with three cycles per $10 \mathrm{~min}$ run cut-off. The first functional volume in the session was aligned to the in-plane anatomical images using rigid body transformations. Automated alignment procedures were followed by careful visual inspection and manual fine-tuning based on anatomical landmarks. Using these transformations, 3D volume time courses were computed in AC-PC space using $1.2 \times 1.2 \times 1.2 \mathrm{~mm}$ voxel size. Additional spatial smoothing $(3 \times 3 \times 3$ $\mathrm{mm}$ ) was applied to the $3 \mathrm{D}$ volume time courses. 3D motion correction with 6 degrees of freedom was done by registering all EPI volumes to their previous volume in the session.

\subsection{Behavioral data analysis}

The behavioral data was analyzed using MATLAB (Mathworks) for performance and RT as in humans. In the ASB and ASR tasks, however, the after wrong hand trials were not used to calculate reaction times since, on the contrary of the other trials of these tasks, the monkeys could predict the hand they would need to use in trial after incorrect hand responses.

\section{7 fMRI data analysis}

One GLM was used to estimate BOLD response associated with relevant tasks events. The GLM was performed only for the voxels encompassed in masks adapted to each monkey brain. The regressors were convolved with a hemodynamic response function (HRF) adjusted for the BOLD dynamics in the monkey brain, peaking at 3 seconds (Kagan et al., 2010). 
We used the six combinations of hand or no response (stay, S) and visual field presentation as main regressors to model the response event: LL, LR, RR, RL, SL and SR. The main regressors consisted of a boxcar function with onsets at the stimulus presentation and duration of mean RT for the task, convolved with the HRF. For the ASB and ASR tasks, these regressors did not contain after wrong hand selection trials for the same reason presented for RT-related calculations. In addition to the main regressors, the GLM contained sixteen regressors of no interest: (1-4) wrong use of the hand for each of the four combinations of hand-related stimulus and visual field presentation, (5-8) after wrong hand trials for each of the four combinations of handrelated stimulus and visual field presentation, (9) trial abortion due to gaze fixation break or jaw motion (only for the monkey C) outside the reward period or the intertrial interval, (10) reward delivery, and (11-16) six head motion regressors extracted during fMRI data preprocessing.

The relationship between the different modeled events and brain activity was analyzed in each individual session. Estimates were tested using contrasts $(p<0.05$ uncorrected) to assess significance across sessions. All reported brain activation were corrected for multiple comparisons with family-wise error (FWE) using alphasim ( $\mathrm{p}<0.05)$ using NeuroElf (1000 interactions, $1.5 \mathrm{~mm}$ smoothing kernel).

\section{Results}

\subsection{Behavioral results}

Monkey B responded slower on the most complex task (ASR task) compared to ASB $\left(\mathrm{t}_{16}=2.461, \mathrm{p}<0.05\right)$ and NAS $\left(\mathrm{t}_{13}=3.864, \mathrm{p}<0.01\right)$ tasks. There was no difference on reaction times between the NAS and the ASB tasks $\left(t_{13}=1.973, p>0.05\right.$; Fig. 18A). Monkey $\mathrm{C}$ responded faster on the NAS task than on action selection tasks (ASB: $\mathrm{t}_{14}=-5.986, \mathrm{p}<0.0001 ;$ ASR: $\left.\mathrm{t}_{13}=-4.717, \mathrm{p}<0.001\right)$. There was no difference on reaction times between the two action selection tasks $\left(t_{17}=1.596, p>0.05\right.$; Fig. 18C). Figures $18 \mathrm{~A}$ and $18 \mathrm{C}$ illustrate the reaction time distribution for all correct trials, even though the tests were performed across sessions' averages.

Monkey B was not significantly faster on uncrossed responses compared to crossed responses in the ASB and NAS tasks (ASB: $\mathrm{t}_{9}=-0.339, \mathrm{p}>0.05 ; \mathrm{NAS}: \mathrm{t}_{5}=-0.665$, 
$\mathrm{p}>0.05)$, but the CUD was significantly positive in the ASR task $\left(\mathrm{t}_{8}=-2.266, \mathrm{p}<0.05\right)$ for the comparison across session averages (Fig. 18B).

For the Monkey C, CUD was not significant in the ASR and NAS tasks (ASR: $t_{8}=-$ $0.546, \mathrm{p}>0.05$; NAS: $\left.\mathrm{t}_{5}=-1.268, \mathrm{p}>0.05\right)$, but it was significant in the ASB task $\left(\mathrm{t}_{9}=-\right.$ 2.441, $\mathrm{p}<0.05$ ) for the comparison across sessions averages (Fig. 18D).
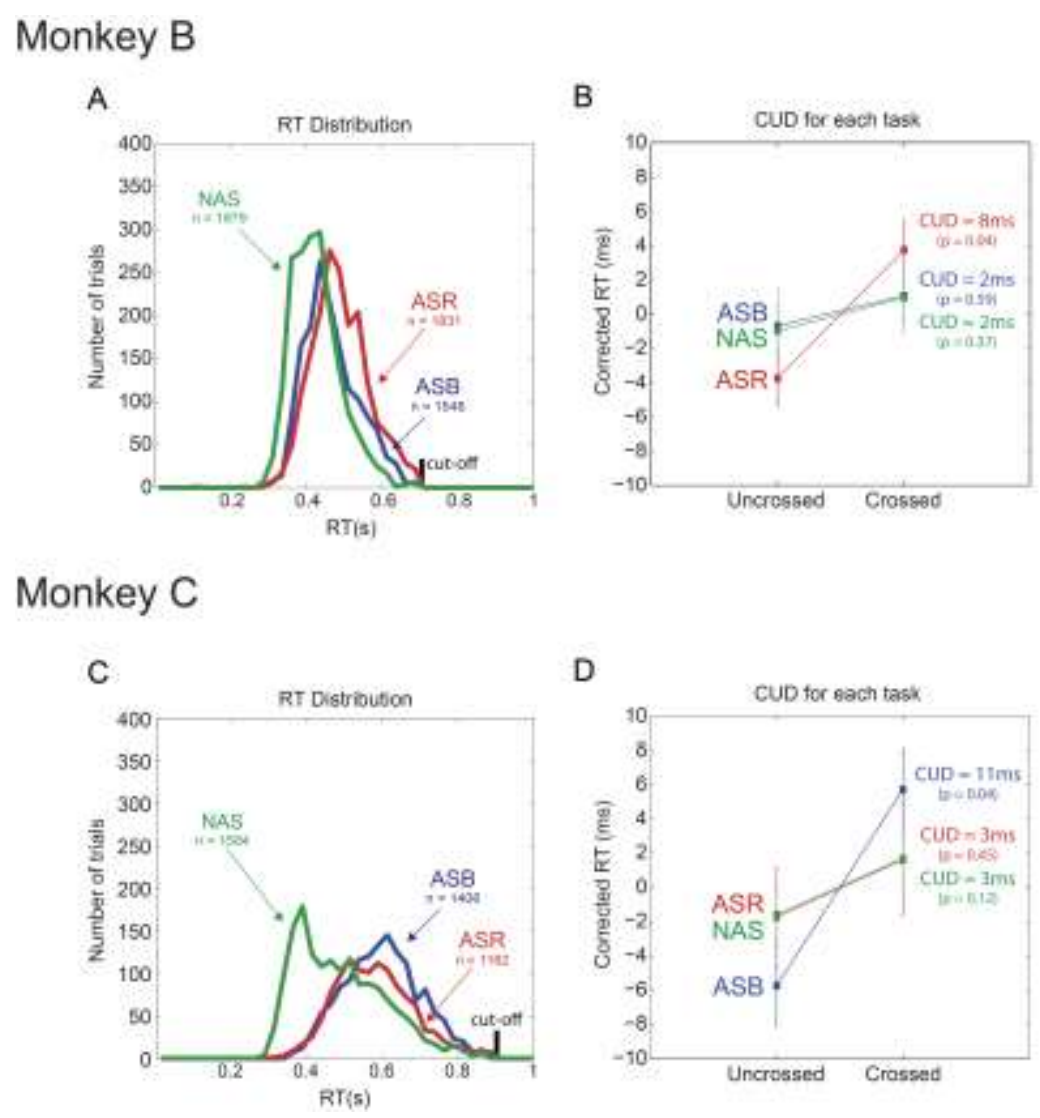

Figure 18. (A and C) Histogram of the RT distribution of all correct trials for the three tasks, and their respective cut-offs. (B and D) Means and standard errors, across sessions, of crossed and uncrossed reaction times for the three tasks, corrected with the average reaction time of each task. No Actions selection task (NAS): green letters and curves. Action Selection Blocked visual field task (ASB): blue letters and curves. Action Selection Random (ASR): red letters and curves.

We also tested CUD in RT for each hand separately (one paired t-test for each hand). For the monkey $\mathrm{B}$, crossed and uncrossed reaction times were not significantly different for any hand during the NAS task (right hand: $\mathrm{t}_{5}=-0.699, \mathrm{p}>0.05$; left hand: $\mathrm{t}_{5}=-0.494, \mathrm{p}>0.05$; Fig. 19A) or during the ASB task (right hand: $\mathrm{t}_{9}=0.625, \mathrm{p}>0.05$; left hand: $\mathrm{t}_{9}=-1.070, \mathrm{p}>0.05$; Fig. 19B). The left hand presented CUD almost 
significant during the ASR task (left hand: $\mathrm{t}_{8}=-2.155, \mathrm{p}=0.06$; right hand: $\mathrm{t}_{8}=-0.726$, p>0.05; Fig. 19C).

Interestingly for the monkey $\mathrm{C}$, while there was positive and almost significant CUD for the left hand in the NAS task $\left(\mathrm{t}_{5}=-2.398, \mathrm{p}=0.06\right)$, the CUD was significantly negative for the right hand $\left(\mathrm{t}_{5}=2.573, \mathrm{p}<0.05\right.$; Fig. 19D). In the ASB task, the CUD was significantly positive for the right hand (right hand: $\mathrm{t}_{9}=-3.448, \mathrm{p}<0.01$; left hand: $\mathrm{t}_{9}=-1.504, \mathrm{p}>0.05$; Fig. 19E). There were no significant differences between crossed and uncrossed reaction times in the ASR task for both hands (left hand: $\mathrm{t}_{8}=-0.796$, $\mathrm{p}>0.05$, right hand: $\mathrm{t}_{8}=0.483, \mathrm{p}>0.05$; Fig. 19F).

\section{Left hand vs Right hand}

\section{Monkey B}
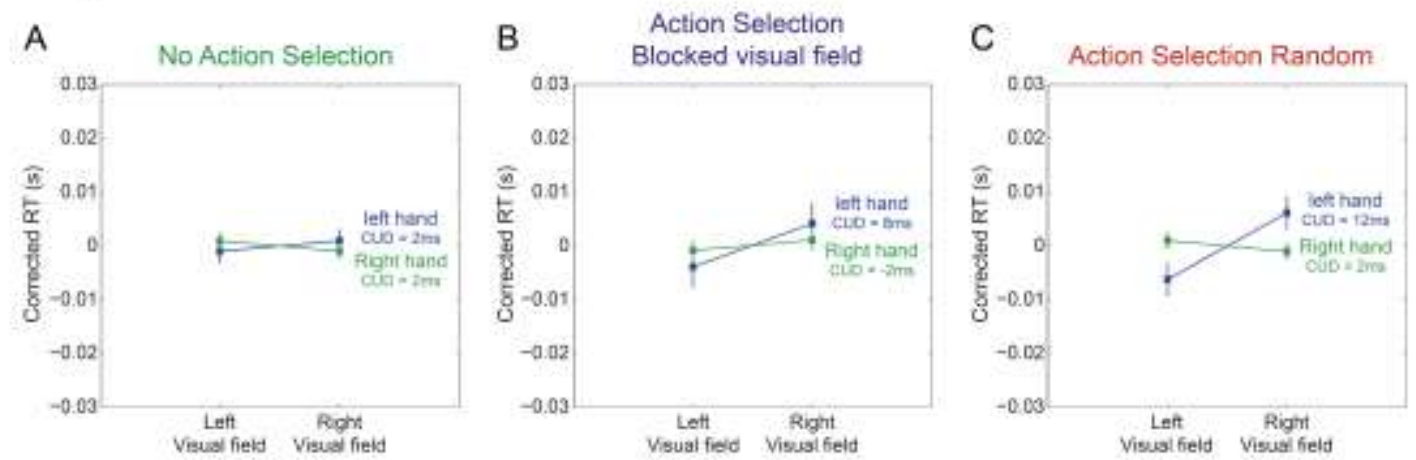

Monkey C
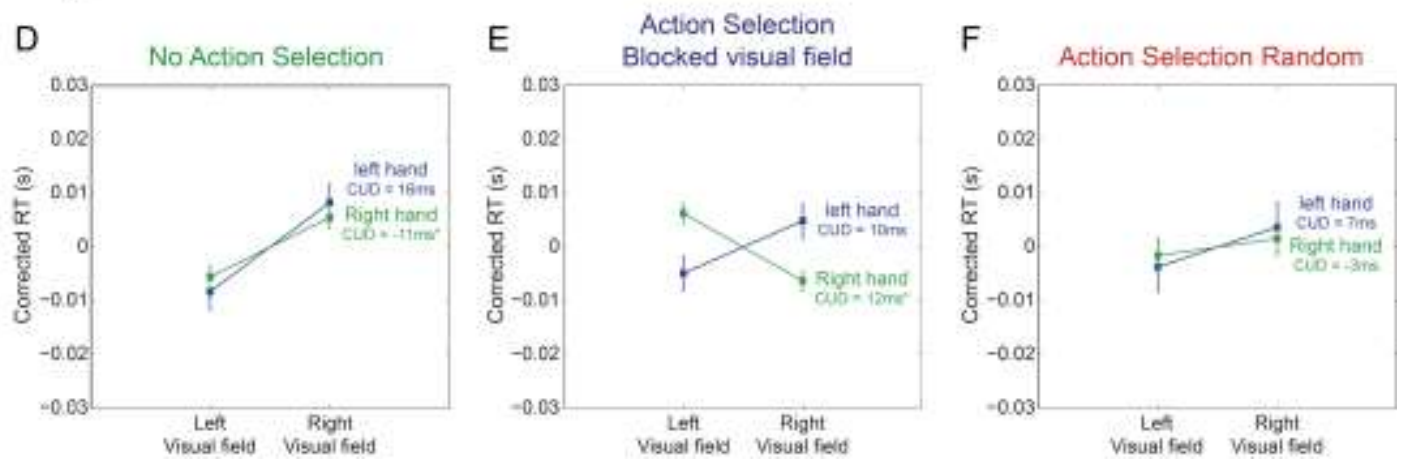

Figure 19. Reaction times for each hand (blue line for the left hand and green line for the right hand) related to each visual field for the (A) NAS, (B) ASB and (C) ASR tasks. Reaction times were corrected with the average reaction time of the respective hand. *CUD t-test, $\mathrm{p}<0.05$.

Although Monkey B presented significant CUD during the ASR task for the comparison across session averages, only 1 individual session presented significant 
CUD across trials. The same occurred for the Monkey C, whose CUD was significant for the ASB task, but only one session presented significant CUD across trials. These results suggest intersession variability for the action selection tasks (Fig. 20).

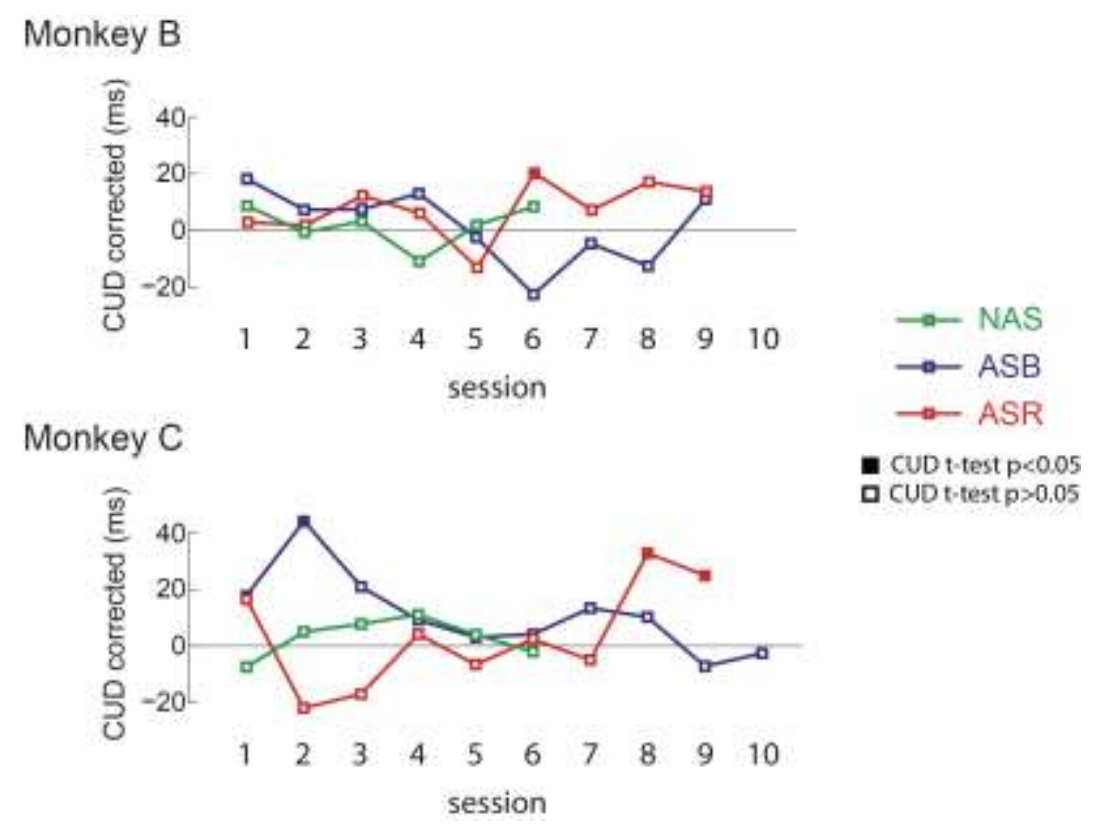

Figure 20. CUD of each session in each task. Filled squares represent significant CUD across trials of the same session (t-test, $\mathrm{p}<0.05)$.

\section{2 fMRI results}

The visual stimulation (i.e. stimulus size and distance from the fixation spot) and the motor requirements (i.e. hand movements) were similar across trials and tasks for the response cues (blue and green squares). The area of the orange triangle matched with the area of the squares, but this cue instructed monkeys to withhold hand responses. Despite the similarity of visual stimulation, differences in trial sequence generated different levels of predictability across tasks (NAS>ASB $>$ ASR), impacting action selection requirements and brain activation patterns.

In general, effector and spatial contralateral tuning were observed in the maps of the three tasks suggesting that, although monkeys responded with the entire arm and thus introduced more noise than the finger movements performed by humans, this noise did not preclude the recording of reliable task-related activity. 
Effector tuning was observed in different areas as the primary motor cortex (M1), the primary sensorimotor cortex (S1), and premotor areas (Fig. 21).
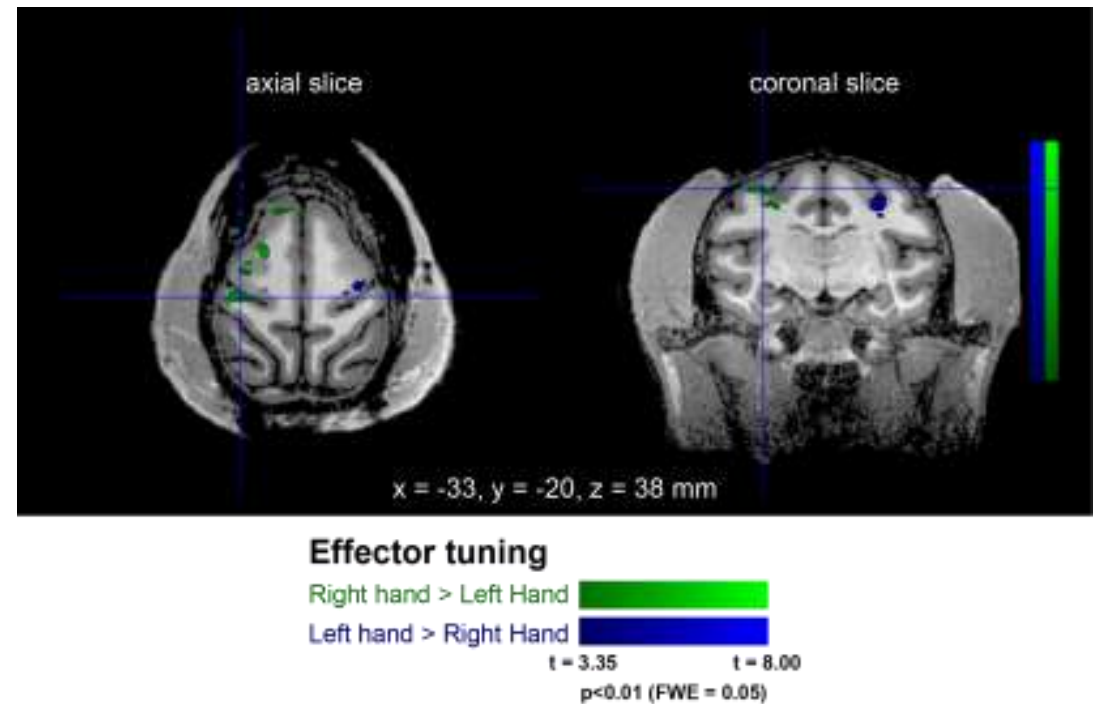

Figure 21. Map showing tuned activity for the hand use in the sensorimotor cortex ( $\mathrm{S} 1: \mathrm{x}=-33, \mathrm{y}=-20$, $\mathrm{z}=38 \mathrm{~mm}$ ), motor cortex (M1) and premotor areas (e.g. area 6, area F2 and area F7). Although this pattern was similar in the three tasks and for both monkeys, this figure illustrates only the effectortuning map of the monkey B during the ASB task.

Space tuning was observed in different areas of the parietal, temporal and occipital cortices (Fig. 22). 


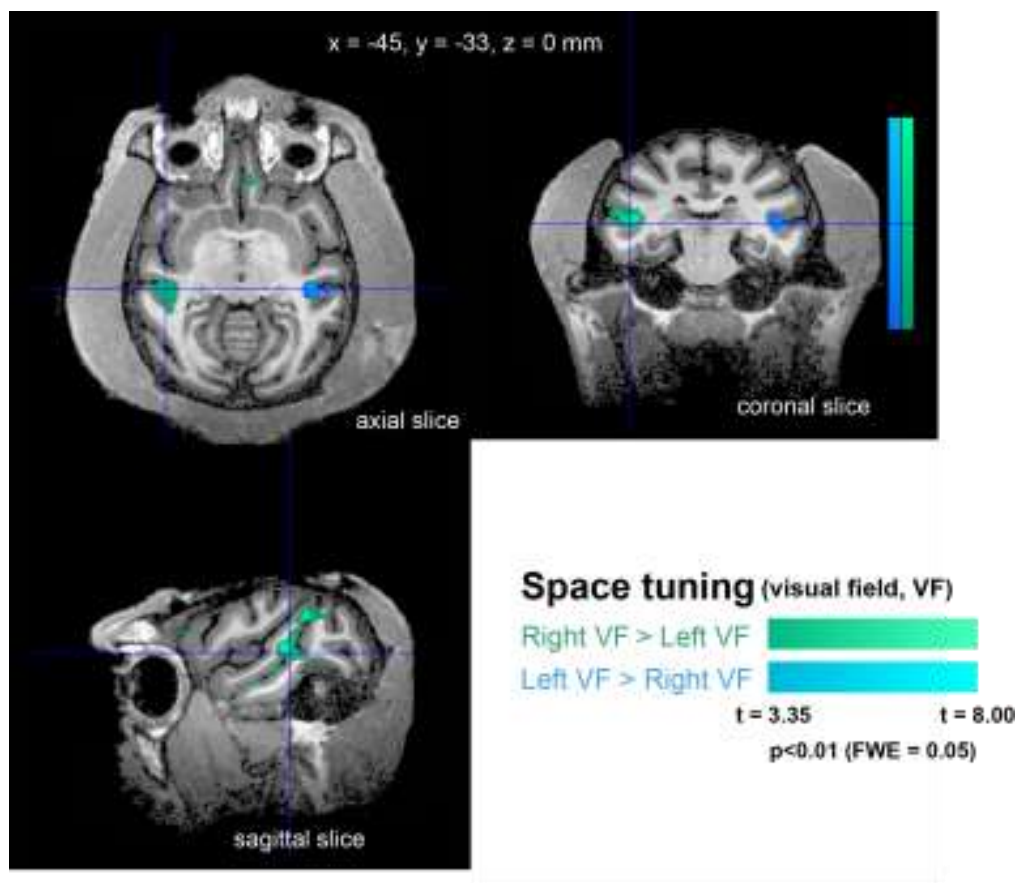

Figure 22. Map showing tuned activity for the visual field presentation in vision-related areas of the parieto-temporal (FST, TPO, MST). Although this pattern was similar in the three tasks and for both monkeys, this figure illustrates only the space-tuning map of the monkey B during the ASR task.

Nevertheless, the maps also contained clusters whose activation reflected the noise due to hand-specific movement artifacts, especially at the edges of the brains (Fig. 23).
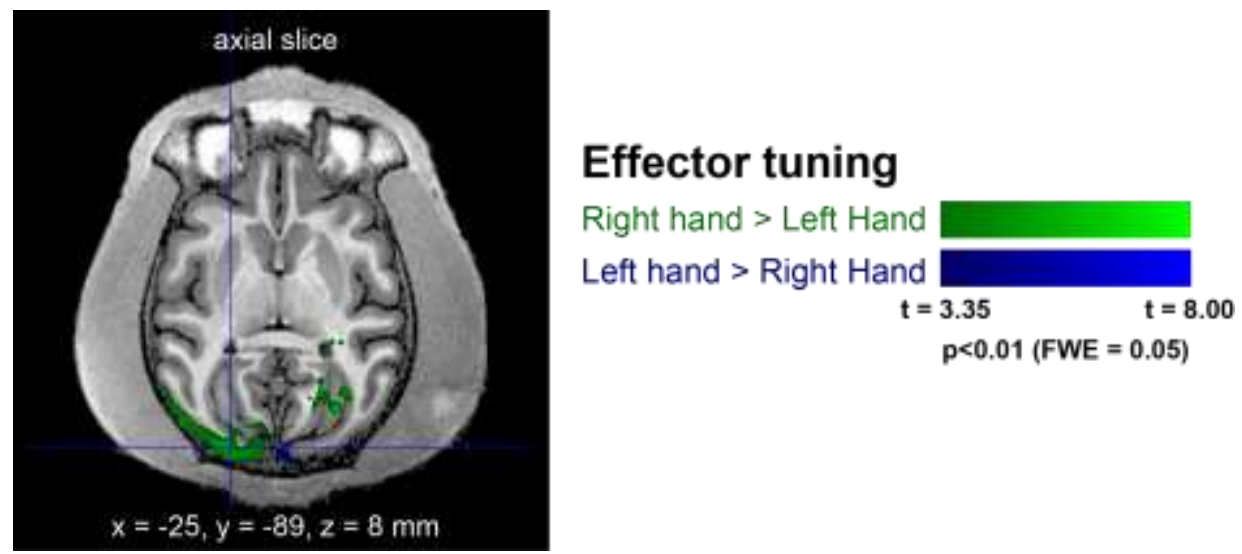

Figure 23. Axial slice showing ipsilateral hand-related (Right hand > Left hand) activation at the posterior edge (primary visual area, V1) of the monkey B brain. Both location and tuning indicated that such activation was related to the noise caused by hand movements and not to BOLD signal. 
Even the CUD contrast (LL+RR vs $L R+R L)$, which is presumably free of handspecific response-related noise (because hand responses were balanced between the conditions), seemed to contain noise activation. Moreover, contrary to the human CUD maps, activations showed different patterns depending on the threshold we applied.

According to our exploratory analysis, several robust differences between crossed and uncrossed activations did not survive a higher threshold ( $\mathrm{p}<0.001$ uncorrected). The CUD map under the adopted threshold ( $\mathrm{p}<0.05$ uncorrected) was more extensive but, conversely, included false positives (for example, activations at the edges of the brain). Considering both undermining results (false positives and false negatives), we decided to select a set of activated clusters using the lower threshold maps (Supplementary Tables 4-9) to investigate CUD-related activity patterns in amplitudes of beta values.

Contrary to what we predicted, and unlike the results reported for humans, some areas were more active during uncrossed responses than during crossed responses for both monkeys. We found this pattern in all the tasks, representing the first robust interspecies difference in brain activity: positive and negative CUD-related activity for monkeys and only positive CUD-related activity for humans. Positive and negative effects in the ROI analysis can be seen as positive and negative t-values in the Supplementary Tables 4-9. These t-values correspond to paired t-tests performed between crossed and uncrossed beta values ( $L L+R R$ vs. $L R+R L)$ across sessions.

Figures 25 and 26 exemplify one chosen area for each monkey (monkey B and monkey C, respectively) in each task (NAS, ASB and ASR). We plotted the four response-related predictors (LL, LR, RL and RR) and the "stay cue" predictors (SL and SR, for stay cues presented in the left and right visual field, respectively). The stay cue beta values provided extra information about the area's function in the visuomotor processing. For example, we interpret the activity in cerebellum as highly tuned to motor responses (positive beta values for the response-related predictors) and not tuned to visual information (negative values for the conditions which did not require motor response; Fig. 25C). Moreover, the activity in the cerebellum during the ASR task presented both hand ( $L L+L R>R R+R L)$ and CUD $(R L+L R>L L+R R)$ tuning, as found for humans during the same task. 
Both CUD-related patterns, positive and negative, reflected robust (t-vales highly different from zero, see Supplementary Tables 4-9) crossed-uncrossed differences in BOLD signal (for positive CUD see Fig. 25A; for negative CUD see Fig. 24B). The activity pattern in the left ventral intraparietal area (left VIP; Fig. 24B) exemplifies the negative CUD in activity found only for monkeys. The right caudate has the activity pattern of an area involved in interhemispheric communication to favor crossed responses (i.e. symmetrical [no hand or space tuning] and positive CUD; Fig. 24A).

\section{Monkey B}
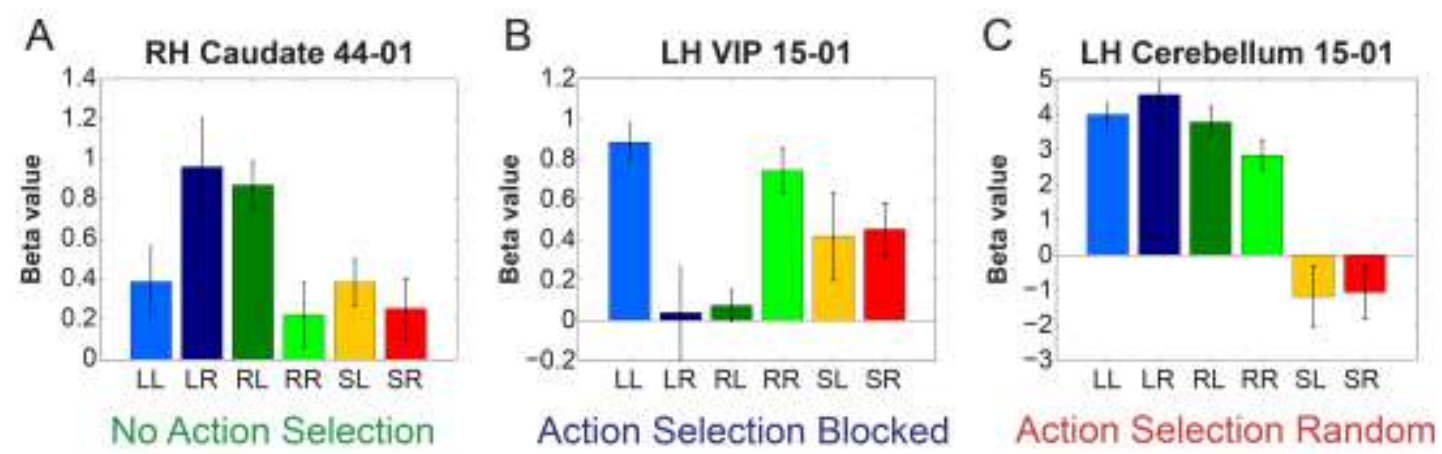

Figure 24. Monkey B. Six main regressors' beta values (means and standard errors) of example areas of each CUD-related task map. (A) Right caudate presenting positive CUD during the NAS task. (B) Left ventral intraparietal area (VIP) presenting negative CUD during the ASB task. (C) Left cerebellum presenting hand and CUD tuning during the ASR task.

The activity in the left supplementary motor area (SMA) of monkey $\mathrm{C}$ during the NAS task (Fig. 25A) presents similar pattern (CUD and hand tuning) as found in the reported cerebellar activity of monkey B during the ASR task (Fig. 24C), emphasizing the motor-related function of this area. The activity of the left parietal area (POa) and the left visual area $4(\mathrm{~V} 4)$ revealed an specific pattern of negative CUD together with the absence of space tuning despite the high beta values found for the predictors related to the stay cue (for the ASB task see Fig. 25B; for the ASR task see Fig. 25C). 


\section{Monkey C}

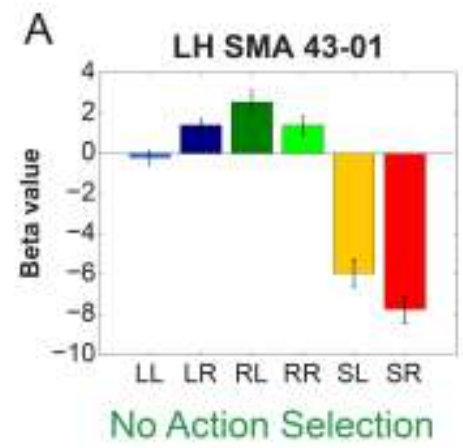

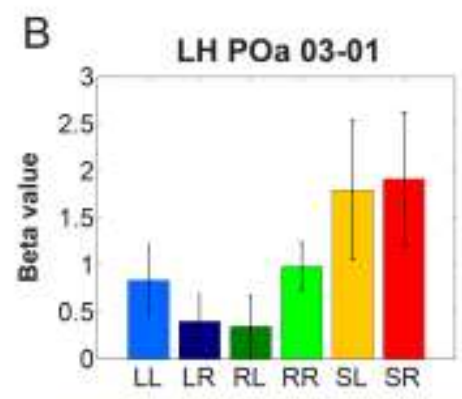

Action Selection Blocked

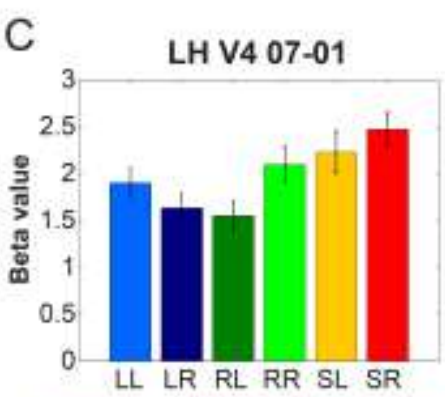

Action Selection Random

Figure 25. Monkey C. Six main regressors' beta values (means and standard errors) of example areas of each CUD-related task map. (A) Left supplementary motor area (SMA) presenting hand and CUD tuning during the NAS task. (B) Left parietal area (POa) presenting negative CUD during the ASB task. (C) Left visual area 4 (V4) presenting negative CUD during the ASR task.

Next, we contrasted the beta values of all response-related predictors between the tasks to investigate if some areas were more or less active in a certain task independently on its activation pattern (i.e. independently if the area presented CUD, hand or space tuning). Differences across tasks were not consistent between the two monkeys. For example, although the activation in the cerebellum was consistently higher when the monkey B was performing the ASR task compared to the other two tasks (Fig. 26A and 26B), and although the mapped areas were generally more active when monkey B was performing action selection tasks compared with the NAS task (e.g. left putamen Fig. 26C), this pattern did not repeat for monkey C (Fig. 27).

\section{Monkey B}

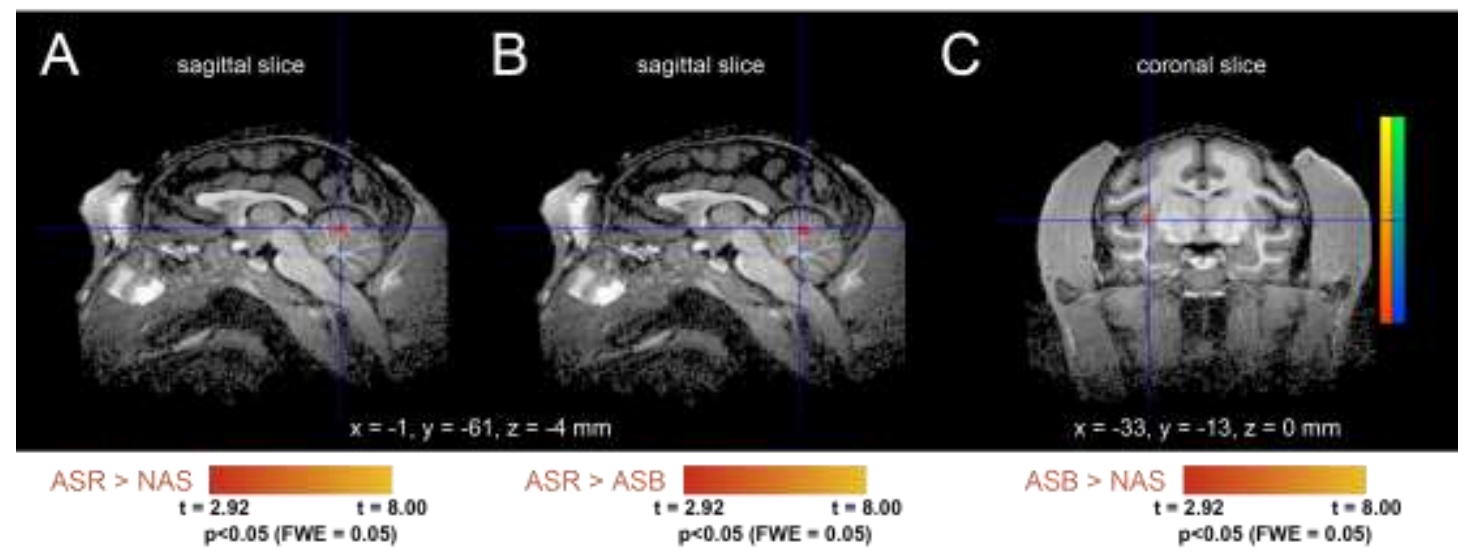

Figure 26. Monkey B. Slices showing differences in brain activity across the tasks. (A) Sagittal slice showing that the cerebellum was more active during the ASR task compared to the NAS task. (B) 
Sagittal slice showing that the cerebellum was more active during the ASR task compared to the ASB task. (C) Coronal slice showing that the left putamen was more active during the ASB task compared to the NAS task.

Monkey $\mathrm{C}$ did not present differences between the action selection tasks (ASB vs ASR) and, although a region in the superior temporal sulcus (sts) was more active during the ASR task compared to the NAS task (Fig. 28A), the parietal area (PO) was more active when no action selection was required (NAS task) than during the ASB task (Fig. 27B), suggesting that the reported task differences for the two monkeys may represent individual differences or noise.

\section{Monkey C}

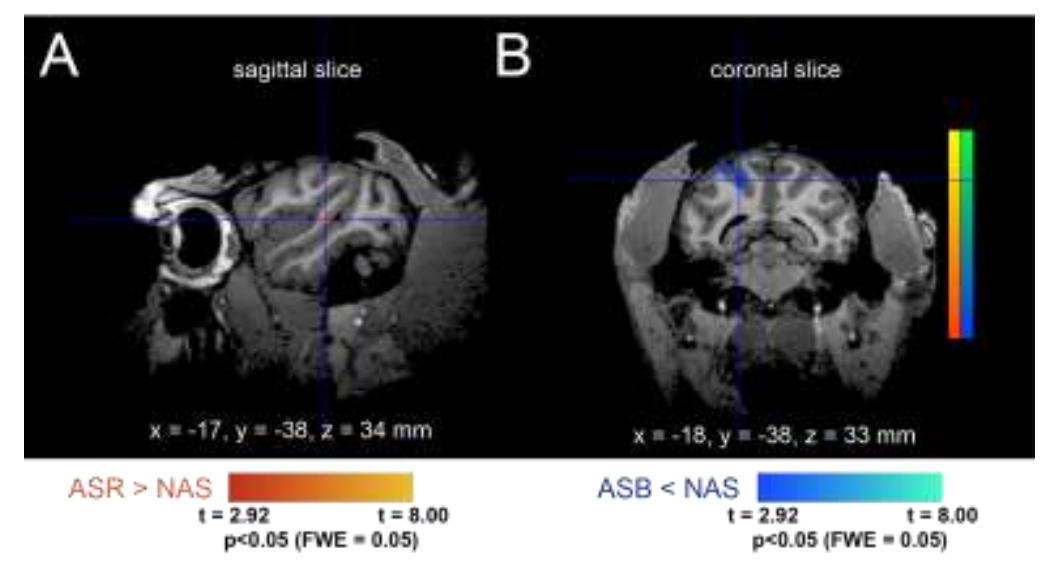

Figure 27. Monkey C. Slices showing differences in brain activity across the tasks. (A) Sagittal slice showing that the left superior temporal sulcus (sts) was more active during the ASR task compared to the NAS task. (B) Coronal slice showing that the left parietal area (PO) was more active during the NAS task compared to the ASB task.

\section{Direct interspecies behavioral comparisons}

Humans performed better in the action selection tasks (ASB and ASR) than monkeys ( $\mathrm{p}<0.001$ for all comparisons across species). The performance in the NAS task, on the other hand, was more similar between the species. Although it was significantly different between humans and monkey B $(\mathrm{p}<0.05)$, it was not significantly different between humans and monkey B ( $>0.05)$ and the difference was smaller compared to the action selection tasks (Fig. 28A). These results suggest that interspecies 
differences decreased when movements could be planned in advance. The performance did not vary between the two monkeys in all three tasks.

The monkey $\mathrm{B}$ was faster than humans and the monkey $\mathrm{C}$ during the two action selection tasks $(\mathrm{p}<0.05)$. The reaction times of humans and the monkey $\mathrm{C}$ were not statistically different when action selection was required ( $>00.05)$. In the NAS task, on the other hand, humans were faster than both monkeys $(\mathrm{p}<0.001)$, representing the only clear interspecies difference in reaction times (Fig. 28B).

Finally, both species did not present CUD in reaction time when no action selection was required $(\mathrm{p}>0.05)$. In the ASB task, the significant CUD of humans and monkey $\mathrm{C}$ were not statistically different $(\mathrm{p}>0.05)$, and only humans had significantly higher CUD than the (nonexistent) CUD of monkey B ( $\mathrm{p}<0.05)$. In the ASR task, humans had higher CUD than both monkeys $(\mathrm{p}<0.001)$. These results suggest that in general, although we found CUD for monkeys during action selection tasks, the interhemispheric transfer time associated to these tasks was longer for humans (Fig. 28C). 
A

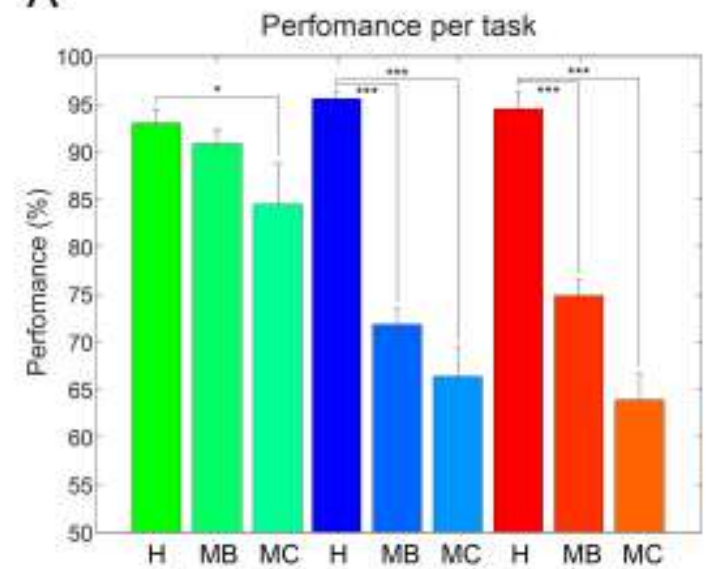

B

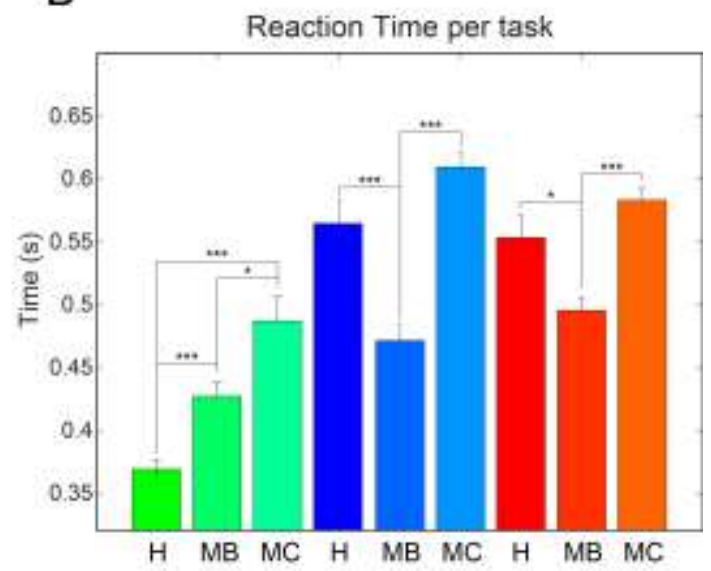

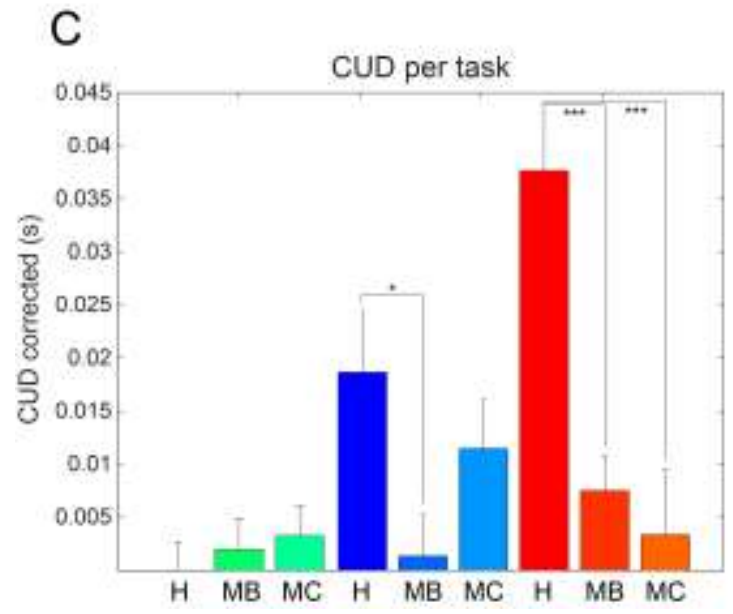

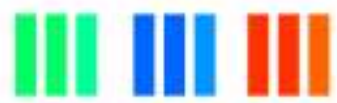

NAS ASB ASR

$\mathrm{H}=$ humans

$\mathrm{MB}=$ monkey $\mathrm{B}$

$\mathrm{MC}=$ Monkey $\mathrm{C}$

Figure 28. (A) Mean and standard errors of performance per task. (B) Mean and standard errors of reaction time per task. (C) Mean and standard errors of CUD per task. Each task is represented by one color: blue bars for the ASB task, green bars for the ASR task and green bars for the NAS task. The bars assigned to humans (H), monkey B (MB) and monkey (MC) have slight brightness variations. *ttest, $\mathrm{p}<0.01$ and $* * *$ t-test, $\mathrm{p}<0.001$.

\section{Discussion}

Fourteen human subjects and two rhesus monkeys performed speeded manual responses to lateralized visual cues while they kept fixating their gaze in the center of a screen inside the MRI scanner. They performed different tasks in different days (monkeys performed each task in multiple and separate days).

Due to the contralateral organization of the brain of primates, different combinations between visual field presentation and hand response determined two conditions: crossed responses, which required interhemispheric transfer of information; and uncrossed responses, which did not require communication between the two brain 
hemispheres. Crossed responses should be slower than uncrossed (Crossed Uncrossed Difference, CUD) because they rely on interhemispheric transfer of information (Poffenberger, 1912).

We hypothesized that, since stimulus-response spatial compatibility (SRSC) effects are only expected in choice tasks (Pellicano et al., 2013) and, therefore, coupled with action selection, the increased uncertainty of the upcoming response requirements would result in longer CUD. This assumption was confirmed since, in humans, the CUD in RT increased with the randomization of the trial sequence, being significant in both species only when action selection was required.

The longest CUD for the monkey B also occurred during the ASR task, but the significant CUD for the monkey $\mathrm{C}$ occurred during the task with intermediate level of trial sequence randomization, the ASB task. Nevertheless, since the most general predicted pattern for CUD in RT - longer CUD during action selection tasks - was found in both species, we suggest that differences between the two monkeys might reflect intersubject variation, which might be present in both species. For example, the human subjects 4, 9 and 12 presented longer CUD during the ASB task compared to the other two tasks. It is also important to emphasize that, as hypothesized, on average CUD in RT was positive in both species during the three tasks.

The direct interspecies behavioral comparison revealed that, when there was significant CUD in RT, these differences were longer in humans. The human brain is, on average, at least four times bigger than the rhesus monkey brain (HerculanoHouzel, 2009) and thus requires longer fibers for interhemispheric connections. According to Aboitiz et al. (2003), the increase in fiber diameter, and consequently in transmission speed, is not enough to compensate for the increased long interhemispheric distances. In this context, the general longer CUD in humans might be result of increased distances between two brain hemispheres compared to monkeys. However, this suggestion might be challenged by the performance comparison between the species and by comparison between the different CUDrelated activation patterns across the tasks in humans (see section 7.2). 


\subsection{Interspecies differences in hemispheric specialization}

Hemispheric specialization is another brain feature that could cause interspecies differences in interhemispheric processing. Although there are multiple lines of evidence that hemispheric asymmetry is not exclusive of humans (Ocklenburg and Güntürkün, 2012), and that it is also present in monkeys (Hamilton and Vermeire, 1988; Jason et al., 1984), the human brain is more lateralized than the monkey brain (Aboitiz et al., 2003; Kagan et al., 2010; Oleksiak et al., 2010; Wey et al., 2013). Nevertheless, in the present study, we did not find clear differences in hemispheric specialization between the species. In humans, when the average CUD in RT was significant (action selection tasks), it was fairly symmetrical between the hands. Nevertheless, four human subjects presented asymmetrical CUD patterns in RT (positive in one hand and negative in the other, significant or not) during the ASB task, five during the NAS task and three during the ASR task. None of the subjects presented such pattern in all tasks. The two monkeys presented positive averaged CUD across sessions (significant or not) for the left hand in all the tasks, whereas the right hand presented negative CUD (significant or not) during the NAS (monkey C), ASB (monkey B) and ASR (monkey C) tasks. The most robust and unexpected result was the significant negative CUD $(-11 \mathrm{~ms})$ for the right hand of the monkey $\mathrm{C}$ during the No Action Selection task. Due to the lack of systematic differences between the species, we attribute the variability in the results to intersubject variation more than to differences between these two primate species.

The comparison of activation patterns between the hemispheres in humans revealed an opposite pattern compared to the one described in previous Poffenberger-related imaging studies with humans (Table 1). While the most consistent finding across the five studies mentioned in the Table 1 was the positive CUD activation in the right precuneus (Iacoboni et al., 2004; Marzi et al., 1999; Tettamanti et al., 2002); in the present study, the CUD map at lower threshold for the NAS task included only areas from the left parietal lobule (Supplementary Fig. 1). This was the most robust interhemispheric asymmetry in the CUD maps of humans considering all tasks. This pattern might result from differences between our Poffenberger-like task (NAS task) and the ones presented in the Table 1 addressed in the section 7.4. 


\subsection{Differences in CUD-related activation patterns and RT across the tasks}

Interestingly, the strongest asymmetry in the CUD maps occurred during the same task (NAS) in which we found ipsilateral space tuning in the right frontal cortex. We suggest that such ipsilateral processing provided representations of visual information from both visual fields in the right hemisphere - as suggested by Mesulam (1999) and Kagan et al. (2010) - facilitating interhemispheric communication and decreasing the CUD in RT to non-significant levels. Commonly, but unlike the original study by Poffenberger (1912), behavioral and slow event-related fMRI studies were performed intermixing left- and right-sided light flashes to avoid visual attention to be anchored in a specific location of the visual field (Iacoboni et al., 2004). We suggest that the ipsilateral representation of visual information in the frontal right hemisphere (e.g. dlPFC) was possible because both hand use and visual field presentation were blocked during the NAS task. Therefore, our results suggest that the more predictable the upcoming response requirements, the earlier (i.e. more visual) is the crossed information.

The CUD map of the ASB task was mainly characterized by the fronto-lateral activations. Even at higher threshold, the CUD activations during this task partially overlapped with the ipsilateral space tuned activation of the NAS task. We interpret this overlap as a demonstration that the inferior frontal gyrus was responsible for crossing similar (probably visual) information in both tasks, since the visual field presentation of lateralized stimulus was equally blocked in both of them. By knowing which hand they should use and where the stimulus would be presented, subjects could prepare the information transfer before the stimulus presentation during the NAS task; but during the ASB task, this transfer preparation could be only partial because, although subjects were attending to only one visual field, they could not predict if the crossed pathway would be useful or not. The stimulus color (and consequently the hand to be used) determined if the proper response was crossed or uncrossed. Thus, we suggest that during the ASB task, the fronto-lateral areas were involved in crossing the information only after having access to the requested motor planning. Nevertheless, we propose that fronto-lateral areas were not involved in the action selection itself, since they were not present in the Action Selection map. Areas from the dorsal parietal cortex (such as precuneus, SPL, S1 and M1) and dACC, on the contrary, were more active when action selection was requested. In fact, dACC 
was present in both maps (the CUD map for the ASB task and the Action Selection Map). The neural activity of this area was associated with conflict monitoring and behavioral adjustments (Kerns et al., 2004). Thus, its CUD-related activation during the ASB task might reflect the inhibition of incompatible responses. This adjustment would be necessary to avoid motor planning conflicts between the brain hemispheres, and might be part of the SRSC effects predicted for the task (De Jong et al., 1994).

Finally, since both tasks, ASR and ASB, required action selection, the difference of CUD in RT between these tasks must be caused by the more spread (i.e. both visual fields) spatial attention during the ASR task. CUD-related activation in IPL, SPL and S1 overlapped with effector-tuned activations during this task. We interpret these results as a demonstration that, when the hand use and the visual field presentation were most unpredictable, the transfer of (excitatory or inhibitory) interhemispheric information occurred later compared to more predictable interhemispheric communication, and thus were encoded by motor-related areas. The association of crossed information with motor processing during the ASR task was further supported by the overlap between CUD and motor tuning maps also in the cerebellum. In this context, SRSC effects would need to be stronger since the inhibition of incompatible responses would occur when the motor processing is more advanced.

Furthermore, in monkey B the cerebellum was clearly more active during the ASR task than during ASB or NAS tasks, and was present in the CUD maps of both action selection tasks (Supplementary Tables 5 and 6). Once again, this pattern was not repeated in monkey $\mathrm{C}$, who had cerebellar activity associated with crossed uncrossed differences in the NAS and ASB tasks. Nevertheless, altogether these results indicate that the cerebellum is involved in interhemispheric communication when action selection is required, especially when subjects are most uncertain about where the stimulus will be presented and how they should respond to this stimulus, implicating the cerebellum in motor adjustments also when interhemispheric transfer is required.

The comparison in CUD-related activation patterns and RT across the three tasks suggests that the content of the information transferred between the brain hemispheres varied according to how predictable was the upcoming response requirements. The more unpredictable the hand use and visual field presentation, the later the stage of the processing (i.e. more motor) is engaged in the interhemispheric communication. Although the RT cut-off during the NAS task was $300 \mathrm{~ms}$ earlier than during action 
selection tasks, the performance of human subjects did not change across the tasks. Monkeys, on the other hand, performed worse during action selection tasks, suggesting that, instead of differences in brain size, the CUD in RT was longer in humans because they delayed the exchange of relevant information between the hemispheres up to the point when a properly selected response could be implemented without affecting the performance. Our findings also suggest that unpredictability did not increase the interhemispheric transfer time per se, but only postponed it to support the exchange of motor information. In fact, if the interhemispheric transfer time of each task would depend exclusively on the differences between the transmission time of the involved areas, the ASB task should present the longest CUD in RT, since the fibers which connect the fronto-lateral areas (most present in the CUD map of the ASB task) between the hemispheres consist of thin slow-conducting axons (Fig. 2; Aboitiz et al., 2003).

\subsection{Poffenberger paradigm vs the NAS task: why we did not find CUD in RT?}

In opposition to different studies that tested interhemispheric transfer time using the Poffenberger paradigm (for review see Marzi et al., 1991), we did not find significant crossed uncrossed difference in RT during the NAS task for any of the species. We attribute the absence of CUD effects in our Poffenberger-like task to two factors: (a) the longer intervals between the response periods (caused by the slow event-related fMRI design we adopted), and (b) to motor response contingencies. Robust CUD in RT during fMRI experiments was mainly found when experimenters used blocked designed tasks (e.g. Tettamanti et al., 2002), while non-significant CUD was found in experiments with slow event-related design (e.g. Martuzzi et al., 2006). Moreover, contrary to simple button presses typically used in Poffenberger-related tasks (Marzi et al., 1991), during our experiments both humans and monkeys needed to release "rest buttons", reach "target buttons" and come back to the rest buttons in order to complete the required response. The increased cognitive and motor requirements is evident in our task since the average human reaction time during the NAS task is around $100 \mathrm{~ms}$ slower than reported by the meta-study of Marzi et al. (1991). We propose that under more complex response contingencies, the small CUD in RT (e.g. $4 \mathrm{~ms}$ ) is diluted across the two response conditions. 


\subsection{Negative CUD in brain activation}

The most striking difference between humans and monkeys in the present experiment was the presence of negative activations exclusively in the CUD maps of monkeys. Even when we performed a fixed-effects analysis (which is more permissive that a random-effects approach) in the first phase of our data analysis, it was clear that almost entire the human brain was more active during crossed responses as compared to uncrossed responses. The absence of negative CUD activation was also evident in the random-effects analysis of humans, whose maps were less extensive and allowed us to locate task-specific areas responsible for putative interhemispheric communication, or increased task demands.

Monkeys, on the contrary, presented robust negative CUD in brain activation. It could be suggested that negative CUD activations were exclusive for monkeys because, by being more impulsive than humans, it was more difficult for them to inhibit nonrequired crossed responses. Additionally, the lower performance of monkeys during action selection tasks could support this suggestion. But, according to previous studies, congruent (uncrossed) responses, and not crossed responses, are automatically activated (De Jong et al., 1994; Pellicano et al., 2013). In this context, the inhibition of those responses should still result in positive CUD patterns (i.e. higher activation for crossed responses). Thus, this interspecies difference requires a more general modification of the task representation. In the present experiment, the visual cues were associated with hand or finger movements towards buttons that subjects were not visualizing. Therefore, all tasks required some level of abstract representation. The higher performance of humans in the action selection tasks (ASB and ASR) suggests that they could comprehend better some of the task requirements and that speeded action selection processes are better performed by humans.

Another important difference was that the task for humans did not contain the "stay cue" (the orange triangle which instructed monkeys to withhold their motor response). This cue was utilized to increase the chance of failure because of impulsive responses. When there are only two response options (right or left hand), monkeys can respond impulsively and still guarantee performance of at least 50\%. By including a third condition (stay cue), we decreased their chance level performance to $33 \%$, making 
them to avoid more impulsive reactions to the "go cues" (i.e. peripheral blue or green square cues). Although negative CUD activations were not always associated with high beta values for the "stay cue" conditions, the existence of such pattern (e.g. Fig. 24B and Fig. 25C) might represent the involvement of some brain areas in processes which inhibit exclusively crossed responses as consequence of a generalization of the inhibitory requirements associated with the stay cue. For instance, when the "stay cue" was presented, these areas would be involved in the general inhibition of motor responses of both hemispheres, which should include, therefore, crossed and uncrossed responses. As consequence of such imprinted pattern, when uncrossed responses were required, these areas would still inhibit the non-required crossed response.

This and other potential differences between the species cannot be resolved using our data. Therefore, we suggest that an extra experiment is necessary to elucidate the differences or similarities between the two species. Patel et al. (2015) compared the attention networks of humans and monkeys in order to understand how evolution changed those networks. Among the eight human subjects used for the interspecies comparisons, three received additional training after the initial scanning sessions and before completing the data collection. By doing this, Patel et al. (2015) were able to compare human behavioral and fMRI results before and after overtraining and, by doing so, match the overtraining effects between the species. Overtraining undermines comparisons between species because animals can learn non-adaptive behaviors and generalize this learning to other representations of the task (Oleksiak et al., 2010). For example, Stamm et al. (1977) reported the influence of hand-specific training on the memory representation of the spatial location of a cue. We believe that a follow up experiment in which few human subjects would be extensively trained in a task containing the all three cues (green and blue squares and the orange triangle) and imaged in different steps of experiment, would help us to elucidate the results that can pertain both to intersubject variation and interspecies differences. Among main findings, we should test if the faster CUD in RT and the negative CUD activations in monkeys are indeed caused by differences between the species.

However, let us for the moment take our findings of the negative CUD at the face value, and assume that they indeed represent a real signature of neuronal processing (or at least its indirect BOLD fMRI correlate). This is not the only difference between 
species we have found. A parallel study in our lab have found that during spatial saccadic decisions, brain activity corresponding to single target instructed trials is smaller than the frontoparietal activity corresponding to the two target choice trials, while the opposite is true in monkeys (humans: choice > instructed, monkeys: choice $<$ instructed). We attributed this difference to summation patterns in less contralateral human spatial representations, and putative inhibition due to competition between contralateral and ipsilateral options in more contralateral monkey representations (Schmidt-Samoa et al., 2012). In the context of crossed-uncrossed responses, one possibility might be that the suppressed crossed activity reflects a stronger interhemispheric competition between response options, which is not present when only one hemisphere implements an uncrossed response.

\subsection{Organization of visuomotor representations}

Since our main research question addresses CUD effects in RT and brain activation, we focused on the interspecies comparison on these aspects of the data. Nevertheless, the visuomotor components of the reaching responses required by our tasks are almost unexplored in fMRI studies, despite the great number of invasive electrophysiological studies in monkeys and imaging studies in humans addressing this topic (e.g. in monkeys Buneo et al., 2002; Gregoriou and Savaki, 2003; Fattori et al., 2010; Kalaska

et al., 1997; Raos et al., 2004; in humans Connolly et al., 2003; Prado et al., 2005; Cavina-Pratesi et al., 2010). This presents another important aspect of our data that warrants further systematic analysis.

Nelissen and Vanduffel (2011) and Premereur et al. (2015) are the only published works which investigated reaching-related activity in macaque brain using a technique (fMRI) that allows direct comparison of results with studies in humans. Nelissen and Vanduffel (2011) showed higher activation in parietal and frontal areas (such as anterior intraparietal area, rostral inferior parietal lobule, somatosensory areas, ventral premotor area F5 and the hand field of F1) during "grasping" compared to "reaching". However, they did not address hand-specific encoding (monkeys were always reaching with one hand), and the relationship between hand and space encoding. Since monkeys performed the tasks in the dark, areas related to visuallyguided manual responses (such as V6A and F2) did not present clear grasp-related 
activity. Moreover, since in our experiments monkeys and humans saw visual cues which instructed reaching responses towards buttons they were not visualizing, we will be able to distinguish between visual and motor representations previously conflated due to the guidance aspect of the visual cues in direct visually-guided tasks (Grol et al., 2007; Raos et al., 2004). In Premereur et al. (2015) monkeys performed two tasks which required the same attention to detect the go cue and the target, but different effectors: eyes or hands to perform visually-guided saccades or reaching. Despite task similarities, two largely segregated cortical networks were activated depending on the effector that was used to respond. In the context of these two experiments - Nelissen and Vanduffel (2011) and Premereur et al. (2015) - further analyses of our data can identify, in addition to interhemispheric interactions, speciesspecific representations of (indirectly) visually-guided reaching activity, and compare the activation patterns with the described patterns for direct visually-guided saccades and reaches. In addition, left/right space-specific vs. left/right hand-specific activation patterns can be analyzed.

\section{Summary}

Despite methodological limitations, we were able to demonstrate that the level of predictability primates have about the upcoming response requirements influences interhemispheric communication by adding action selection and SRSC effects to the brain processing. In this context, the unpredictability would not increase the interhemispheric transfer time per se, but would delay the exchange of relevant information between the hemispheres until the point when the proper response can be implemented without decreasing performance. In monkeys, due to their impulsivity, failure in delaying the responses until the point in which the transfer of interhemispheric information would guarantee the same performance, or smaller brain size (and consequently interhemispheric distances), resulted in shorter CUD in RT for action selection tasks.

Further experiments (i.e. in overtrained humans), more rigorous data inspection and analysis of the principle findings, as well as more advanced analysis approaches (e.g. functional connectivity) should be performed in order to corroborate, and further investigate: (a) the suggested inhibitory mechanism that caused negative CUD activations, (b) optimal approach in statistical mapping and extracting the activation 
patterns, especially in the monkey datatset, and (c) the direction of the interhemispheric transfer of information. 


\section{Supplementary information}

Cluster tables for humans. The clusters of voxels were extracted using NeuroElf (www.neuroelf.net) and the brain areas identified according to the atlas of the Talairach Project at the Research Imaging Institute of the University of Texas Health Science Center San Antonio (http://www.talairach.org/applet.html). Since, in some cases, different clusters of voxels were located in the same brain area, we identified the clusters with numbers (last column of the cluster tables) in order to crossreference the information presented in the figures of the Results sections and the following supplementary tables.

The cluster tables were extracted from the lower threshold CUD maps (Supplementary Figures 1-3). Areas present also in the higher threshold maps (Figures 8-10) are highlighted with starts and the orange text color.

\section{Supplementary Table 1}

Table of clusters with significant CUD activity in the NAS task for the threshold $\mathrm{p}<0.01$ (black text) and $\mathrm{p}<0.001$ (orange text)

\begin{tabular}{|c|c|c|c|c|c|c|c|}
\hline & Name & Hemisphere & $\mathrm{x}$ & $\mathrm{y}$ & $\mathrm{Z}$ & t value & Cluster \\
\hline 1 & Posterior Cingulate Cortex & Left & -16 & -48 & 21 & 5.98 & 0101 \\
\hline 2 & dorsal Anterior Cingulate Cortex & Left & -15 & 11 & 31 & 5.24 & 0102 \\
\hline 3 & Sub-Gyral & Left & -24 & -31 & 37 & 4.49 & 0103 \\
\hline 4 & Middle Frontal Gyrus & Left & -24 & 11 & 34 & 4.46 & 0104 \\
\hline 5 & dorsal Premotor Cortex & Left & -24 & -4 & 46 & 4.28 & 0105 \\
\hline 6 & dorsal Anterior Cingulate Cortex & Left & -15 & -10 & 37 & 3.95 & 0106 \\
\hline 7 & dorsal Premotor Cortex & Left & -15 & 11 & 46 & 3.73 & 0108 \\
\hline 8 & White matter (Cingulate) * & Left & -16 & -48 & 21 & 3.72 & 0109 \\
\hline 9 & Medial Frontal Gyrus & Left & -15 & 2 & 58 & 3.28 & 0114 \\
\hline 10 & Medial Frontal Gyrus* & Right & 15 & 11 & 46 & 4.99 & 0201 \\
\hline 11 & Sub-Gyral* & Right & 21 & -4 & 55 & 4.54 & 0202 \\
\hline 12 & Cingulate Gyrus & Right & 24 & 2 & 34 & 4.14 & 0203 \\
\hline 13 & Superior Frontal Gyrus* & Right & 6 & 11 & 49 & 4.11 & 0204 \\
\hline 14 & Medial Frontal Gyrus & Left & 0 & 26 & 46 & 4.09 & 0205 \\
\hline 15 & dorsal Anterior Cingulate Cortex & Right & 15 & 8 & 34 & 4.03 & 0206 \\
\hline 16 & Cingulate Gyrus & Right & 21 & 17 & 25 & 3.78 & 0207 \\
\hline 17 & Sub-Gyral & Right & 24 & -16 & 49 & 3.5 & 0208 \\
\hline 18 & dorsal Premotor Cortex & Right & 3 & 2 & 58 & 3.31 & 0209 \\
\hline 19 & Anterior Lobe & Left & -24 & -55 & -29 & 4.16 & 0301 \\
\hline 20 & Uvula & Left & -12 & -67 & -26 & 4.04 & 0302 \\
\hline 21 & Pyramis* & Right & 12 & -70 & -29 & 4.84 & 0401 \\
\hline 22 & Precuneus & Right & 12 & -58 & 52 & 4.4 & 0501 \\
\hline
\end{tabular}




$\begin{array}{llcccccc}23 & \text { Precuneus } & \text { Right } & 18 & -46 & 37 & 4.24 & 0502 \\ 24 & \text { Precuneus } & \text { Right } & 12 & -61 & 43 & 3.98 & 0503 \\ 25 & \text { Precuneus } & \text { Left } & -9 & -55 & 52 & 4.24 & 0601 \\ 26 & \text { Precuneus } & \text { Left } & -18 & -52 & 49 & 3.69 & 0602 \\ 27 & \text { Postcentral Gyrus } & \text { Right } & 33 & -25 & 31 & 3.68 & 0702\end{array}$

\section{Supplementary Table 2}

Table of clusters with significant CUD activity in the ASB task for the threshold $\mathrm{p}<0.01$ (black text) and $\mathrm{p}<0.001$ (orange text)

\begin{tabular}{|c|c|}
\hline & Name \\
\hline 1 & dorsolateral Prefrontal Cortex* \\
\hline 2 & Lingual Gyrus* \\
\hline 3 & Thalamus* \\
\hline 4 & Culmen* \\
\hline 5 & Insula* \\
\hline 6 & Thalamus* \\
\hline 1 & Thalamus* \\
\hline 8 & Lentiform Nucleus \\
\hline 9 & Claustrum* \\
\hline 10 & Caudate \\
\hline 11 & Culmen* \\
\hline 12 & Insula* \\
\hline 13 & Lingual Gyrus \\
\hline 14 & Declive \\
\hline 15 & Lentiform Nucleus \\
\hline 16 & Culmen \\
\hline 17 & Cingulate Gyrus* \\
\hline 18 & dorsal Premotor Cortex* \\
\hline 19 & Anterior Cingulate* \\
\hline 20 & Pre Supplementary Motor Area* \\
\hline 21 & Supplementary Motor Area * \\
\hline 27 & dorsal Anterior Cingulate Cortex \\
\hline 23 & dorsal Anterior Cingulate Cortex \\
\hline 24 & Primary Motor Cortex \\
\hline - & dorsal Anterior Cingulate Cortex" \\
\hline 26 & dorsal Anterior Cingulate Cortex \\
\hline 27 & dorsal Premotor Cortex \\
\hline 28 & Lentiform Nucleus \\
\hline 29 & ventral Premotor Cortex \\
\hline 30 & Claustrum \\
\hline 31 & Superior Temporal Gyrus \\
\hline 32 & Lentiform Nucleus \\
\hline 02 & Lentiform Nucleus \\
\hline & Inferior Frontal Gyrus \\
\hline & Primary Motor Cortex \\
\hline
\end{tabular}

\begin{tabular}{|c|c|c|c|c|c|}
\hline Hemisphere & $\mathrm{x}$ & $\mathrm{y}$ & $\mathrm{z}$ & t value & Cluster \\
\hline Right & 45 & 14 & 28 & 7.04 & 0101 \\
\hline Right & 18 & -40 & 1 & 6.73 & 0102 \\
\hline Right & 15 & -22 & 4 & 6.31 & 0103 \\
\hline Right & 3 & -58 & 1 & 6.09 & 0104 \\
\hline Right & 39 & 11 & 10 & 4.86 & 0105 \\
\hline Right & 24 & -22 & 4 & 4.66 & 0106 \\
\hline Right & 6 & -16 & 7 & 4.57 & 0107 \\
\hline Right & 24 & -10 & -2 & 4.54 & 0108 \\
\hline Right & 36 & 8 & 1 & 4.52 & 0109 \\
\hline Right & 18 & -7 & 16 & 4.51 & 0110 \\
\hline Right & 3 & -49 & -2 & 4.34 & 0111 \\
\hline Right & 36 & 20 & 10 & 4.3 & 0112 \\
\hline Right & 21 & -64 & -2 & 3.91 & 0115 \\
\hline Right & 15 & -64 & -14 & 3.9 & 0116 \\
\hline Right & 18 & 5 & 1 & 3.56 & 0119 \\
\hline Left & -6 & -67 & -5 & 3.51 & 0120 \\
\hline Left & -12 & 11 & 34 & 7.92 & 0201 \\
\hline Right & 3 & 2 & 52 & 5.61 & 0202 \\
\hline Left & -6 & 8 & 25 & 5.47 & 0203 \\
\hline Right & 12 & -4 & 52 & 4.99 & 0204 \\
\hline Right & 15 & -13 & 55 & 4.79 & 0205 \\
\hline Right & 3 & -1 & 34 & 4.53 & 0206 \\
\hline Right & 3 & 14 & 34 & 4.48 & 0207 \\
\hline Right & 27 & -13 & 46 & 4.38 & 0208 \\
\hline Left & -12 & 5 & 43 & 4.26 & 0209 \\
\hline Right & 3 & -1 & 43 & 3.86 & 0211 \\
\hline Right & 30 & -4 & 49 & 3.86 & 0212 \\
\hline Left & -24 & -16 & -2 & 4.78 & 0301 \\
\hline Left & -54 & 5 & 13 & 4.66 & 0302 \\
\hline Left & -33 & 5 & 10 & 4.57 & 0303 \\
\hline Left & -54 & -4 & 4 & 4.00 & 0304 \\
\hline Left & -21 & 11 & 10 & 3.87 & 0305 \\
\hline Left & -18 & -1 & 1 & 3.87 & 0306 \\
\hline Left & -45 & 8 & 19 & 3.62 & 0309 \\
\hline Left & -51 & -1 & 28 & 3.52 & 0310 \\
\hline
\end{tabular}




\begin{tabular}{|c|c|c|c|c|c|c|c|}
\hline 36 & Precentral Gyrus & Left & -39 & -1 & 28 & 3.48 & 0311 \\
\hline 37 & dorsolateral Prefrontal Cortex* & Right & 36 & 41 & 34 & 6.47 & 0401 \\
\hline 38 & anterior Prefrontal Cortex* & Right & 45 & 41 & 7 & 5.99 & 0402 \\
\hline 39 & dorsolateral Prefrontal Cortex $*$ & Left & -39 & 29 & 37 & 6.1 & 0501 \\
\hline 40 & dorsolateral Prefrontal Cortex & Left & -27 & 20 & 34 & 5.44 & 0502 \\
\hline 41 & dorsolateral Prefrontal Cortex * & Left & -30 & 26 & 46 & 4.53 & 0503 \\
\hline 42 & dorsolateral Prefrontal Cortex * & Left & -36 & 41 & 31 & 4.01 & 0504 \\
\hline 43 & dorsolateral Prefrontal Cortex & Left & -48 & 41 & 22 & 3.59 & 0505 \\
\hline 44 & Precuneus & Left & -21 & -46 & 46 & 4.23 & 0601 \\
\hline 45 & Inferior Parietal Lobule & Left & -48 & -43 & 55 & 3.49 & 0604 \\
\hline 46 & Superior Parietal Lobule & Left & -30 & -55 & 46 & 3.48 & 0605 \\
\hline 47 & Precuneus & Left & -30 & -43 & 43 & 3.43 & 0606 \\
\hline 48 & Inferior Parietal Lobule & Left & -39 & -46 & 46 & 3.37 & 0608 \\
\hline 49 & Superior Parietal Lobule & Left & -27 & -67 & 46 & 3.28 & 0609 \\
\hline 50 & Precuneus* & Right & 24 & -67 & 37 & 4.81 & 0701 \\
\hline 51 & Precuneus & Right & 18 & -67 & 22 & 4.09 & 0702 \\
\hline 52 & Precuneus & Right & 15 & -67 & 52 & 3.51 & 0703 \\
\hline 53 & Cuneus & Right & 18 & -82 & 31 & 3.13 & 0704 \\
\hline 54 & Middle Temporal Gyrus & Left & -39 & -61 & 1 & 3.61 & 0802 \\
\hline 55 & Middle Temporal Gyrus & Left & -60 & -46 & 10 & 3.56 & 0804 \\
\hline 56 & Middle Temporal Gyrus & Left & -48 & -55 & 4 & 3.55 & 0805 \\
\hline 57 & Superior Temporal Gyrus & Left & -54 & -37 & 10 & 3.41 & 0807 \\
\hline 58 & Superior Temporal Gyrus & Right & 66 & -22 & 13 & 4.23 & 0902 \\
\hline 59 & Postcentral Gyrus & Right & 66 & -22 & 28 & 4.41 & 0903 \\
\hline 60 & Superior Temporal Gyrus & Right & 60 & -31 & 16 & 3.75 & 0904 \\
\hline 61 & Inferior Parietal Lobule & Right & 51 & -28 & 25 & 3.32 & 0906 \\
\hline 62 & Inferior Temporal Gyrus* & Right & 48 & -49 & 1 & 5.35 & 1001 \\
\hline 63 & Sub-Gyral* & Right & 45 & -40 & -5 & 5.28 & 1002 \\
\hline 64 & Primary Motor Cortex & Left & -15 & -19 & 55 & 4.92 & 1101 \\
\hline 65 & Precentral Gyrus & Left & -30 & -13 & 61 & 4.27 & 1102 \\
\hline 66 & Anterior Lobe & Right & 18 & -43 & -29 & 5.03 & 1201 \\
\hline 67 & Culmen & Right & 18 & -25 & -32 & 4.47 & 1202 \\
\hline
\end{tabular}

\section{Supplementary Table 3}

Table of clusters with significant CUD activity in the ASR task for the threshold $p<0.01$ (black text) and $\mathrm{p}<0.001$ (orange text)

\begin{tabular}{|c|c|c|c|c|c|c|c|}
\hline & Name & Hemisphere & $\mathrm{x}$ & $\mathrm{y}$ & $\mathrm{z}$ & $\mathrm{t}$ value & Cluster \\
\hline 1 & Culmen & Left & -12 & -22 & -20 & 5.95 & 0101 \\
\hline 2 & Culmen* & Right & 12 & -46 & -14 & 5.02 & 0102 \\
\hline 3 & Anterior LobeDentate* & Right & 15 & -49 & -23 & 4.87 & 0103 \\
\hline 4 & Culmen* & Right & 12 & -37 & -17 & 4.57 & 0104 \\
\hline 5 & Culmen & Right & 0 & -52 & -20 & 4.43 & 0105 \\
\hline 6 & Fusiform Gyrus & Right & 48 & -34 & -8 & 4.42 & 0106 \\
\hline 7 & MidbrainRed Nucleus & Left & 0 & -22 & -14 & 4.36 & 0108 \\
\hline 8 & Culmen* & Left & -3 & -40 & -17 & 4.34 & 0109 \\
\hline
\end{tabular}




$\begin{array}{llcccccc}9 & \text { Culmen } & \text { Left } & -18 & -43 & -20 & 4.29 & 0110 \\ 10 & \text { Culmen } & \text { Right } & 24 & -31 & -23 & 3.51 & 0113 \\ 11 & \text { Declive } & \text { Right } & 15 & -61 & -14 & 3.49 & 0114 \\ 12 & \text { Culmen } & \text { Right } & 33 & -34 & -20 & 3.47 & 0116 \\ 13 & \text { Inferior Parietal Lobule* } & \text { Right } & 42 & -43 & 55 & 7.03 & 0201 \\ 14 & \text { Postcentral Gyrus } & \text { Right } & 39 & -25 & 49 & 5.66 & 0202 \\ 15 & \text { Precentral Gyrus } & \text { Right } & 24 & -22 & 61 & 4.85 & 0203 \\ 16 & \text { Superior Parietal Lobule* } & \text { Right } & 33 & -46 & 46 & 4.1 & 0204 \\ 17 & \text { Postcentral Gyrus } & \text { Right } & 45 & -25 & 58 & 4.01 & 0205 \\ 18 & \text { Inferior Parietal Lobule } & \text { Right } & 42 & -52 & 49 & 3.81 & 0206 \\ 19 & \text { Middle Temporal Gyrus* } & \text { Left } & -63 & -22 & -8 & 5.27 & 0301 \\ 20 & \text { Superior Temporal Gyrus* } & \text { Left } & -48 & -13 & -8 & 4.89 & 0302 \\ 21 & \text { Middle Temporal Gyrus* } & \text { Left } & -54 & -22 & -14 & 4.62 & 0303 \\ 22 & \text { Inferior Parietal Lobule* } & \text { Left } & -48 & -37 & 55 & 7.45 & 0401 \\ 23 & \text { Postcentral Gyrus* } & \text { Left } & -51 & -28 & 49 & 5.75 & 0402 \\ 24 & \text { Inferior Parietal Lobule } & \text { Left } & -39 & -49 & 55 & 4.22 & 0403 \\ 25 & \text { Postcentral Gyrus } & \text { Left } & -36 & -28 & 55 & 3.81 & 0404 \\ 26 & \text { Declive } & \text { Right } & 27 & -79 & -20 & 4.71 & 0501 \\ 27 & \text { Declive } & \text { Left } & -3 & -82 & -26 & 4.45 & 0502 \\ 28 & \text { Declive } & \text { Right } & 9 & -73 & -20 & 3.74 & 0503 \\ 29 & \text { Uvula } & \text { Left } & -18 & -79 & -26 & 3.49 & 0504 \\ 30 & \text { Precuneus } & \text { Left } & 0 & -64 & 55 & 4.08 & 0602 \\ 31 & \text { Precuneus } & \text { Left } & -3 & -76 & 46 & 3.6 & 0603 \\ 32 & \text { Cingulate Gyrus } & \text { Left } & -12 & -37 & 37 & 3.59 & 0604 \\ 33 & \text { Superior Frontal Gyrus } & \text { Left } & -6 & -4 & 70 & 4.42 & 0701\end{array}$

Cluster tables for monkeys. Clusters were named according to the Calabrese et al. (2015) MRI+DTI atlas (https://scalablebrainatlas.incf.org/macaque/CBCetal15). Unlike in human data, the clusters were not split in subclusters. Note the negative $\mathrm{t}$ values (dark red text) indicate the decrease for the crossed $v s$ uncrossed comparison (crossed < uncrossed, negative CUD). All areas included in the Supplementary tables 4-9 presented significant difference between crossed and uncrossed beta values across sessions (paired t-test, $\mathrm{p}<0.05$ ).

\section{Monkey B}

\section{Supplemantary Table 4}

Areas with CUD in activity during the NAS task for the monkey B

$\begin{array}{llcccccc} & \text { Name } & \text { Hemisphere } & \mathrm{x} & \mathrm{y} & \mathrm{z} & \mathrm{t} \text { value } & \text { Cluster } \\ 1 & \text { Supplementary motor area (6) } & \text { Right } & 9 & -17 & 36 & 6.27 & 0101 \\ 2 & \text { Parietal area (PEa) } & \text { Left } & -9 & -57 & 22 & 6.88 & 0301 \\ 3 & \text { Somatosensory cortex (3a) } & \text { Right } & 11 & -27 & 46 & 5.57 & 0401\end{array}$




\begin{tabular}{|c|c|c|c|c|c|c|c|}
\hline 4 & Putamen $(\mathrm{Pu})$ & Left & -23 & 7 & -8 & -11.92 & 0501 \\
\hline 5 & Ventral part of area $9 / 46(46 \mathrm{~V})$ & Left & -25 & 29 & 16 & -7.29 & 0601 \\
\hline 6 & Parietal area $(\mathrm{PEa})$ & Right & 39 & -27 & 36 & 7.16 & 0701 \\
\hline 7 & Somatosensory cortex (2) & Left & -11 & -37 & 50 & 6.07 & 0801 \\
\hline 8 & Dorsal part of visual area 4 (V4D) & Right & 45 & -53 & 10 & -11.04 & 1501 \\
\hline 9 & Granular insular cortex (Gl) & Left & -35 & -13 & 10 & -9.05 & 1601 \\
\hline 10 & Visual area 2 (V2) & Right & 19 & -65 & 6 & -15.45 & 2201 \\
\hline 11 & Dorsal part of visual area 4 (V4D) & Left & -57 & -45 & 0 & -6.30 & 3101 \\
\hline 12 & Visual cortex $4(\mathrm{~V} 4)$ & Left & 9 & -9 & 42 & 5.32 & 3301 \\
\hline 13 & Dorsorostral area 6 (F7) & Left & -23 & 11 & 36 & -5.58 & 3701 \\
\hline 14 & Ventral part of visual area $4(\mathrm{~V} 4 \mathrm{~V})$ & Left & -47 & -53 & -6 & -4.39 & 4001 \\
\hline 15 & Dorsal anterior cingulate cortex (24c) & Left & -13 & 3 & 30 & -10.10 & 4301 \\
\hline 16 & Caudate nucleus $(\mathrm{Cd})$ & Right & 13 & 1 & 12 & 5.88 & 4401 \\
\hline 17 & Temporal parietooccipital area (TPO) & Right & 53 & -23 & -2 & 5.35 & 4601 \\
\hline 10 & Dorsal part of visual area 4 (V4D) & Right & 53 & -47 & 0 & 3.13 & 4801 \\
\hline & Caudal part of the Parietal area (PEC) & Right & 11 & -61 & 36 & 5.04 & 5301 \\
\hline
\end{tabular}

\section{Supplemantary Table 5}

Areas with CUD in activity during the ASB task for the monkey B

\begin{tabular}{|c|c|c|c|c|c|c|c|}
\hline & Name & Hemisphere & $\mathrm{x}$ & $\mathrm{y}$ & $\mathrm{Z}$ & $\mathrm{t}$ value & Cluster \\
\hline 1 & Visual area 1 (V1) & Right & 37 & -69 & 2 & -5.81 & 0201 \\
\hline 2 & Parietal area $(\mathrm{PEa})$ & Left & -27 & -29 & 36 & 7.77 & 0301 \\
\hline 3 & Caudate nucleus (Cd) & Right & 11 & 21 & 14 & 4.92 & 0401 \\
\hline 4 & Caudate nucleus $(\mathrm{Cd})$ & Left & -9 & 11 & 10 & 6.31 & 0601 \\
\hline 5 & Cingulate cortex (24b) & Right & 1 & -7 & 28 & 4.59 & 0701 \\
\hline 6 & Putamen $(\mathrm{Pu})$ & Right & 35 & -1 & 0 & 5.18 & 0801 \\
\hline 7 & Dysgranular insular cortex (Dl) & Left & -37 & -1 & -8 & 5.55 & 0901 \\
\hline 8 & Cerebellum (Cb) & Left & -25 & -49 & -12 & 4.86 & 1101 \\
\hline 0 & Medial part temporal area (TE) & Left & -59 & -25 & -6 & 4.86 & 1201 \\
\hline 10 & Dorsal part of visual area 4 (V4D) & Left & -59 & -39 & 8 & -4.99 & 1301 \\
\hline 11 & Area 45B & Left & -43 & 13 & 18 & -5.52 & 1401 \\
\hline 12 & Ventral intraparietal area (VIP) & Left & -21 & -33 & 24 & -6.28 & 1501 \\
\hline 13 & Visual area 2 (V2) & Right & 19 & -53 & 0 & 6.55 & 2301 \\
\hline
\end{tabular}

\section{Supplemantary Table 6}

Areas with CUD in activity during the ASR task for the monkey B

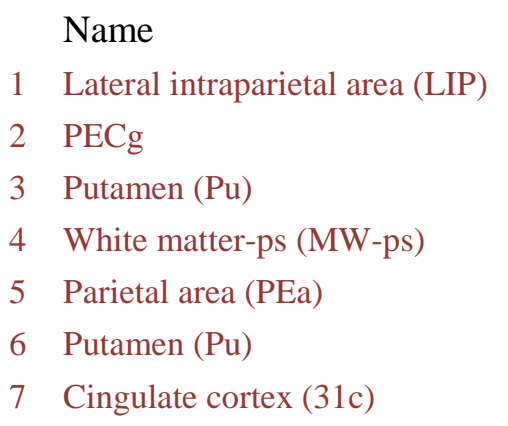

$\begin{array}{cccccc}\text { Hemisphere } & \mathrm{X} & \mathrm{y} & \mathrm{z} & \mathrm{t} \text { value } & \text { Cluster } \\ \text { Right } & 13 & -55 & 34 & -7.68 & 0201 \\ \text { Right } & 15 & -29 & 26 & -5.52 & 0301 \\ \text { Right } & 21 & 5 & -8 & -4.10 & 0601 \\ \text { Left } & -13 & 31 & 12 & -5.09 & 0801 \\ \text { Left } & -41 & -19 & 28 & -4.80 & 0901 \\ \text { Left } & -19 & 1 & 12 & -4.79 & 1001 \\ \text { Left } & -1 & -39 & 28 & -7.83 & 1201\end{array}$




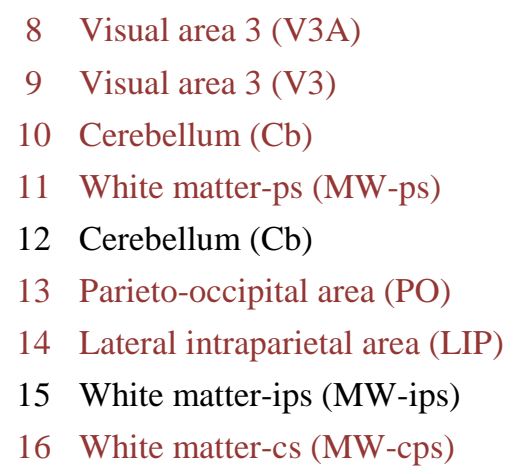

$\begin{array}{cccccc}\text { Left } & -35 & -63 & 30 & -4.16 & 1301 \\ \text { Right } & 11 & -19 & 34 & -4.86 & 1401 \\ \text { Left } & -1 & -67 & 4 & -5.52 & 1501 \\ \text { Right } & 3 & 11 & 18 & -4.55 & 1601 \\ \text { Right } & 5 & -47 & -4 & 4.90 & 1701 \\ \text { Right } & 3 & -71 & 16 & -6.22 & 1801 \\ \text { Right } & -15 & -57 & 26 & -9.16 & 1901 \\ \text { Right } & 17 & -53 & 20 & 5.40 & 2101 \\ \text { Left } & -23 & -11 & 28 & -4.46 & 2301\end{array}$

\section{Monkey C}

\section{Supplemantary Table 7}

Areas with CUD in activity during the NAS task for the monkey C

\begin{tabular}{|c|c|c|c|c|c|c|c|}
\hline & Name & Hemisphere & $\mathrm{x}$ & $\mathrm{y}$ & $\mathrm{Z}$ & t value & Cluster \\
\hline 1 & Anteroventral part of area $8(8 \mathrm{AV})$ & Right & 30 & 16 & 30 & 6.76 & 0101 \\
\hline 2 & White matter-cg (WM-cg) & Left & -2 & 12 & 22 & -14.4 & 0201 \\
\hline 3 & Visual area $1(\mathrm{~V} 1)$ & Right & 24 & -72 & 14 & -8.33 & 0301 \\
\hline 4 & Secondary somatosensory cortex (S2) & Right & 46 & -18 & 16 & -5.51 & 0401 \\
\hline 5 & Visual area $3(\mathrm{~V} 3 \mathrm{a})$ & Right & 28 & -8 & 26 & -6.33 & 0501 \\
\hline 6 & White matter-ips (WM-ips) & Left & -14 & -48 & 20 & -9.47 & 0601 \\
\hline 7 & Parieto-occipital area (PO) & Right & 10 & -64 & 26 & 5.12 & 0801 \\
\hline 8 & Orbital proisocortex (Opro) & Left & -22 & 14 & -2 & -5.63 & 0901 \\
\hline 9 & Secondary somatosensory cortex (S2) & Left & -40 & -24 & 18 & -6.45 & 1301 \\
\hline 10 & Cingulate cortex (31c) & Left & -2 & -24 & 30 & -6.51 & 1901 \\
\hline 11 & Fundus superior temporal sulcus (FST) & Right & 40 & -30 & 4 & 6.87 & 2501 \\
\hline 12 & Temporoparietal cortex (Tpt) & Left & -42 & -40 & 30 & 3.69 & 2601 \\
\hline 13 & Area PGM-31 of cortex (PGM-31) & Left & -4 & -60 & 28 & 5.13 & 2901 \\
\hline 14 & Cingulate cortex (23c) & Right & 8 & -10 & 28 & -4.65 & 3001 \\
\hline 15 & Parietal area $(\mathrm{PEa})$ & Left & -16 & -40 & 38 & 3.53 & 3101 \\
\hline 16 & Cerebellum $(\mathrm{Cb})$ & Right & 0 & -70 & 2 & 5.23 & 3401 \\
\hline 17 & Visual area $2(\mathrm{~V} 2)$ & Left & -28 & -66 & 20 & 3.71 & 3501 \\
\hline 18 & Cerebellum $(\mathrm{Cb})$ & Left & -6 & -54 & -2 & -4.18 & 3801 \\
\hline 19 & Putamen $(\mathrm{Pu})$ & Right & 22 & 4 & 6 & -7.36 & 4001 \\
\hline 20 & Frontal eye field (FEF) & Left & -30 & 6 & 24 & 4.76 & 4201 \\
\hline 21 & Supplementary motor area $(6 \mathrm{M})$ & Left & -2 & 0 & 46 & 3.50 & 4301 \\
\hline 22 & Medial superior temporal area (MST) & Right & 24 & -42 & 22 & -3.76 & 4401 \\
\hline 23 & Dorsocaudal area $6(\mathrm{~F} 2)$ & Right & 12 & 10 & 44 & 3.21 & 4801 \\
\hline
\end{tabular}




\section{Supplemantary Table 8}

Areas with CUD in activity during the ASB task for the monkey C

\begin{tabular}{|c|c|c|c|c|c|c|}
\hline Name & Hemisphere & $\mathrm{x}$ & $\mathrm{y}$ & $\mathrm{z}$ & t value & Cluster \\
\hline 1 Secondary somatosensory cortex (S2) & Left & -6 & -30 & 42 & -6.24 & 0101 \\
\hline Granular insular cortex (Gl) & Left & -34 & -18 & 10 & -8.39 & 0201 \\
\hline External part of the parietal area (POa) & Left & -26 & -36 & 38 & -5.21 & 0301 \\
\hline Cerebellum (Cb) & Right & 8 & -70 & -6 & 5.00 & 0401 \\
\hline
\end{tabular}

\section{Supplemantary Table 9}

Areas with CUD in activity during the ASR task for the monkey C

\begin{tabular}{|c|c|c|c|c|c|c|}
\hline Name & Hemisphere & $\mathrm{x}$ & $\mathrm{y}$ & $\mathrm{z}$ & t value & Cluster \\
\hline Temporoparietal cortex (Tpt) & Left & -54 & -32 & 18 & -4.86 & 0101 \\
\hline Visual area 2 (V2) & Right & 12 & -62 & 8 & 4.40 & 0201 \\
\hline External part of the parietal area (POa) & Left & -16 & -52 & 30 & 4.34 & 0501 \\
\hline Dorsal part of visual area 4 (V4D) & Left & -44 & -42 & 8 & -4.65 & 0701 \\
\hline Fundus superior temporal sulcus (FST) & Right & 26 & -40 & 10 & -7.76 & 0801 \\
\hline Internal part of the parietal area (POa) & Left & -32 & -26 & 26 & -4.13 & 0901 \\
\hline Visual area $1(\mathrm{~V} 1)$ & Left & -28 & -44 & 8 & -4.06 & 1001 \\
\hline Visual area $1(\mathrm{~V} 1)$ & Right & 24 & -66 & -4 & 2.98 & 1101 \\
\hline
\end{tabular}

Lower uncorrected threshold maps. The uncorrected thresholds for the lower threshold maps were $\mathrm{p}<0.01$ (t-test) for the CUD map, and $\mathrm{p}<0.05$ (t-test) for spatial and hand tuning maps. To the contrary of the Figure 8, the expected contralateral spatial tuning was observed using a lower threshold for the space map (Supplementary Fig. 1).

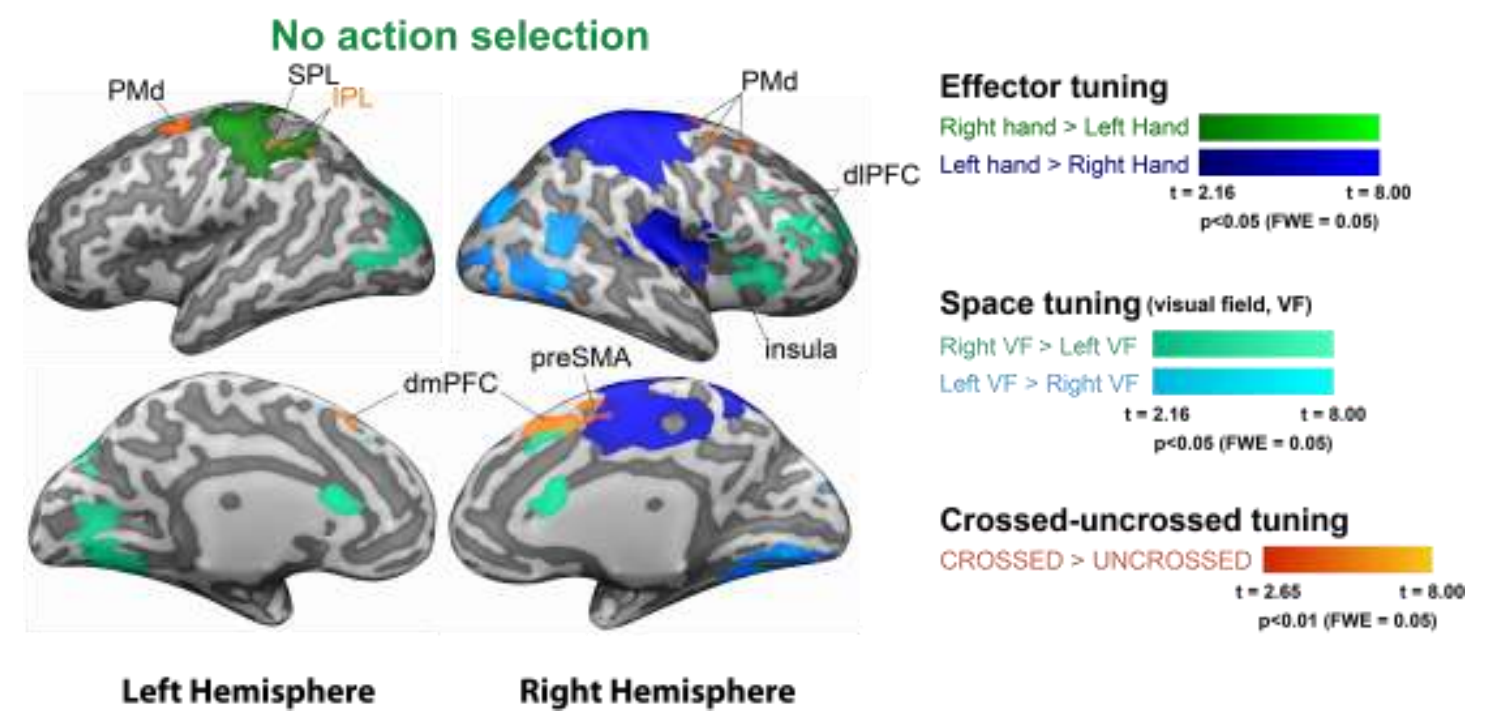

Supplementary Figure 1. Lower threshold maps on the inflated brain for three activation tunings when subjects performed the ASB task. Areas in dark green and blue reflect effector tuning for right and left 
hands, respectively. Areas in light green and blue reflect space tuning for right and left visual fields, respectively. The main contrast (orange) mapped areas more active during crossed compared to uncrossed responses. There was no area more active during uncrossed than during crossed responses.

The CUD map of the ASB task was more symmetrical under lower threshold, showing extensive frontal activation (Supplementary Fig. 2).

\section{Action Selection Blocked visual field}

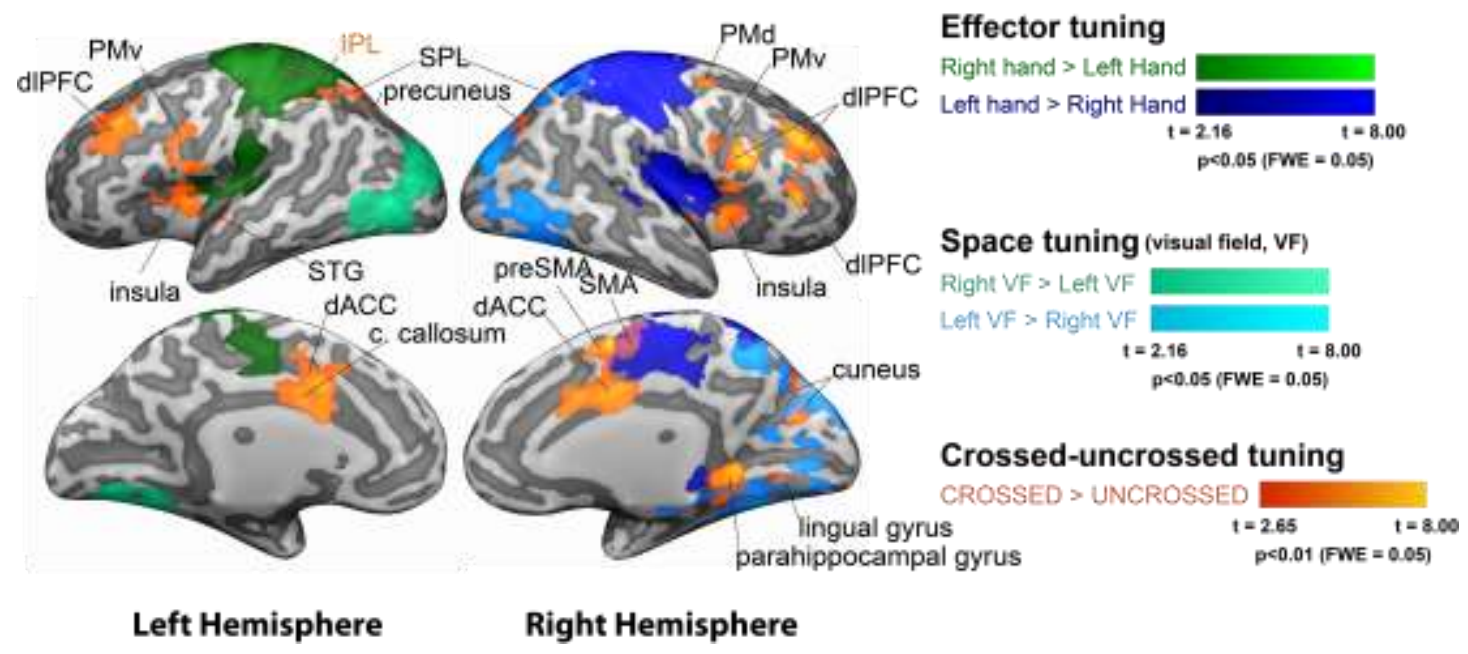

Supplementary Figure 2. Lower threshold maps on the inflated brain for three activation tunings when subjects performed the ASB task. Areas in dark green and blue reflect effector tuning for right and left hands, respectively. Areas in light green and blue reflect space tuning for right and left visual fields, respectively. The main contrast (orange) mapped areas more active during crossed compared to uncrossed responses. There was no area more active during uncrossed than during crossed responses.

The only CUD-related area present on all lower threshold maps is the inferior parietal lobule (IPL; Supplementary Figures 1-3). 


\section{Action Selection Random}

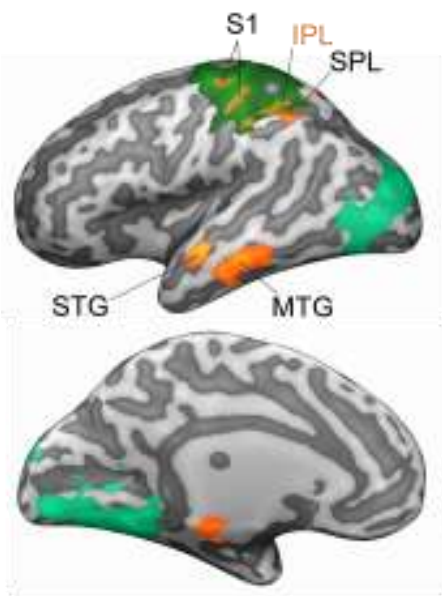

Left Hemisphere

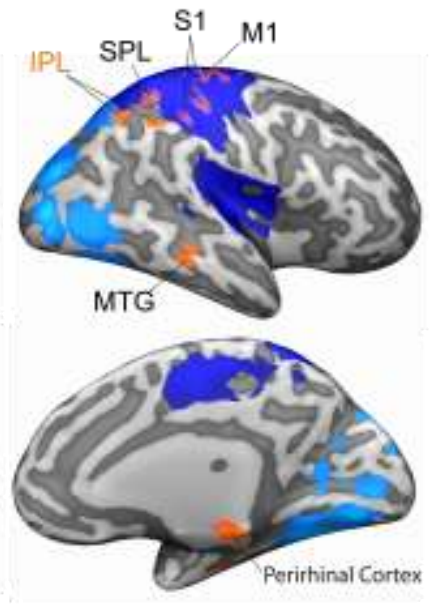

Right Hemisphere

\section{Effector tuning}

Right hand > Left Hand

Left hand > Right Hand

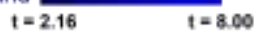

p<0.05 (FWE $=0.05$ )

Space tuning (visual field, VF)

Right WF $>$ Left WF

Left WF s Right WF

$$
\begin{aligned}
& t=2.16 \quad t=8.00 \\
& \text { p } 00.05 \text { (FWE }=0.05 \text { ) }
\end{aligned}
$$

Crossed-uncrossed tuning

CROSSED $>$ UNCROSSED $t=2.65$ $t=8.00$

Supplementary Figure 3. Lower threshold maps on the inflated brain for three activation tunings when subjects performed the ASB task. Areas in dark green and blue reflect effector tuning for right and left hands, respectively. Areas in light green and blue reflect space tuning for right and left visual fields, respectively. The main contrast (orange) mapped areas more active during crossed compared to uncrossed responses. There was no area more active during uncrossed than during crossed responses. 


\section{References}

Aboitiz, F., López, J., \& Montiel, J. (2003). Long distance communication in the human brain: timing constraints for inter-hemispheric synchrony and the origin of brain lateralization. Biological Research, 36(1), 89-99.

Anzola, G. P., Bertoloni, G., Buchtel, H. A., \& Rizzolatti, G. (1977). Spatial compatibility and anatomical factors in simple and choice reaction time.Neuropsychologia, 15(2), 295-302.

Baker, J. T. (2005). Distribution of Activity Across the Monkey Cerebral Cortical Surface, Thalamus and Midbrain during Rapid, Visually Guided Saccades. Cerebral Cortex, 16(4), 447-459. http://doi.org/10.1093/cercor/bhi124

Brandt, T., Marx, E., Sthephan, T., Bense, S., \& Dieterich, M. (2003). Interhemispheric Transmission of Visuomotor. Ann. NY Acad. Sci, 1004(1), 283-288.

Buneo, C. A., Jarvis, M. R., Batista, A. P., \& Andersen, R. A. (2002). Direct visuomotor transformations for reaching. Nature, 416(6881), 632-636.

Byrne, R. A., Kuba, M., \& Griebel, U. (2002). Lateral asymmetry of eye use in Octopus vulgaris. Animal Behaviour, 64(3), 461-468. http://doi.org/10.1006/anbe.2002.3089

Caminiti, R., Ghaziri, H., Galuske, R., Hof, P. R., \& Innocenti, G. M. (2009). Evolution amplified processing with temporally dispersed slow neuronal connectivity in primates. Proceedings of the National Academy of Sciences, 106(46), 19551-19556.

Cavina-Pratesi, C., Monaco, S., Fattori, P., Galletti, C., McAdam, T. D., Quinlan, D. J., ... \& Culham, J. C. (2010). Functional magnetic resonance imaging reveals the neural substrates of arm transport and grip formation in reach-to-grasp actions in humans. The Journal of Neuroscience, 30(31), 10306-10323.

Schmidt-Samoa C., Wilke M., Dechent P., Andersen R.A., Kagan I. (2012) Spatial decision differently activates frontoparietal network in humans and in monkeys. SfN 2012

Chiang, T. C., \& Lavidor, M. (2005). Magnetic stimulation and the crossed uncrossed difference (CUD) paradigm: selective effects in the ipsilateral and contralateral hemispheres. Experimental Brain Research, 160(3), 404-408. http://doi.org/10.1007/s00221-004-2166-9 
Concha, M. L., Burdine, R. D., Russell, C., Schier, A. F., \& Wilson, S. W. (2000). A nodal signaling pathway regulates the laterality of neuroanatomical asymmetries in the zebrafish forebrain. Neuron, 28(2), 399-409.

Connolly, J. D., Andersen, R. A., \& Goodale, M. A. (2003). FMRI evidence for a'parietal reach region'in the human brain. Experimental Brain Research,153(2), 140-145.

Csermely, D., Bonati, B., Lopez, P., and Martin, J. (2011). Is the Podarcis muralis lizard left-eye lateralized when exploring a new environment? Laterality 16, $240-255$.

Dadda, M., Domenichini, A., Piffer, L., Argenton, F., \& Bisazza, A. (2010). Early differences in epithalamic left-right asymmetry influence lateralization and personality of adult zebrafish. Behavioural Brain Research, 206(2), 208-215. http://doi.org/10.1016/j.bbr.2009.09.019

De Jong, R., Liang, C.C., \& Lauber, E. (1994). Conditional and unconditional automaticity: a dual-process model of effects of spatial stimulus-response correspondence. Journal of Experimental Psychology: Human Perception and Performance, 20(4), 731.

Fattori, P., Raos, V., Breveglieri, R., Bosco, A., Marzocchi, N., \& Galletti, C. (2010). The dorsomedial pathway is not just for reaching: grasping neurons in the medial parieto-occipital cortex of the macaque monkey. The Journal of Neuroscience, 30(1), 342-349.

Felleman, D. J., \& Van Essen, D. C. (1991). Distributed hierarchical processing in the primate cerebral cortex. Cerebral cortex, 1(1), 1-47.

Fischer, J., \& Whitney, D. (2009). Precise discrimination of object position in the human pulvinar. Human Brain Mapping, 30(1), 101-111. http://doi.org/10.1002/hbm.20485

Frasnelli, E., Anfora, G., Trona, F., Tessarolo, F., \& Vallortigara, G. (2010). Morphofunctional asymmetry of the olfactory receptors of the honeybee (Apis mellifera). Behavioural Brain Research, 209(2), 221-225. http://doi.org/10.1016/j.bbr.2010.01.046

Gaffan, D., \& Hornak, J. (1997). Visual neglect in the monkey. Representation and disconnection. Brain, 120(9), 1647-1657. 
Goense, J. B. M., Whittingstall, K., \& Logothetis, N. K. (2010). Functional magnetic resonance imaging of awake behaving macaques. Methods, 50(3), 178-188. http://doi.org/10.1016/j.ymeth.2009.08.003

Gregoriou, G. G., \& Savaki, H. E. (2003). When vision guides movement: a functional imaging study of the monkey brain. Neuroimage, 19(3), 959-967.

Grol, M. J., Majdandžić, J., Stephan, K. E., Verhagen, L., Dijkerman, H. C., Bekkering, H., ... \& Toni, I. (2007). Parieto-frontal connectivity during visually guided grasping. The Journal of Neuroscience, 27(44), 11877-11887.

Hamilton, C. R., \& Vermeire, B. A. (1988). Complementary hemispheric specialization in monkeys. Science, 242(4886), 1691.

Herculano-Houzel, S. (2009). The human brain in numbers: a linearly scaled-up primate brain. Frontiers in Human Neuroscience, 3. http://doi.org/10.3389/neuro.09.031.2009

Holmes, C. J., Hoge, R., Collins, L., Woods, R., Toga, A. W., \& Evans, A. C. (1998). Enhancement of MR images using registration for signal averaging. Journal of Computer Assisted Tomography, 22(2), 324-333.

Iacoboni, M. (2004). Interhemispheric visuo-motor integration in humans: the role of the superior parietal cortex. Neuropsychologia, 42(4), 419-425. http://doi.org/10.1016/j.neuropsychologia.2003.10.007

Innocenti, G. M., Aggoun-Zouaoui, D., \& Lehmann, P. (1995). Cellular aspects of callosal connections and their development. Neuropsychologia, 33(8), 961-987.

Jason, G. W., Cowey, A., \& Weiskrantz, L. (1984). Hemispheric asymmetry for a visuo-spatial task in monkeys. Neuropsychologia, 22(6), 777-784.

Kagan, I., Iyer, A., Lindner, A., \& Andersen, R. A. (2010). Space representation for eye movements is more contralateral in monkeys than in humans. Proceedings of the National Academy of Sciences, 107(17), 7933-7938. http://doi.org/10.1073/pnas.1002825107

Kalaska, J. F., Scott, S. H., Cisek, P., \& Sergio, L. E. (1997). Cortical control of reaching movements. Current opinion in neurobiology, 7(6), 849-859.

Kerns, J. G. (2004). Anterior Cingulate Conflict Monitoring and Adjustments in Control. Science, 303(5660), 1023-1026. http://doi.org/10.1126/science.1089910 
Kornblum, S., Hasbroucq, T., \& Osman, A. (1990). Dimensional overlap: cognitive basis for stimulus-response compatibility - a model and taxonomy. Psychological review, 97(2), 253.

Koyama, M., Hasegawa, I., Osada, T., Adachi, Y., Nakahara, K., \& Miyashita, Y. (2004). Functional magnetic resonance imaging of macaque monkeys performing visually guided saccade tasks: comparison of cortical eye fields with humans. Neuron, 41(5), 795-807.

Loosemore, R. G. (2011). The evolution of forebrain contralaterality as a response to eye development: the path of least resistance. Hypotheses in the Life Sciences, $1(1), 11-20$.

Manning, L., \& Thomas-Antérion, C. (2011). Marc Dax and the discovery of the lateralisation of language in the left cerebral hemisphere. Revue Neurologique, 167(12), 868-872. http://doi.org/10.1016/j.neurol.2010.10.017

Martuzzi, R., Murray, M. M., Maeder, P. P., Fornari, E., Thiran, J.P., Clarke, S., ... Meuli, R. A. (2006). Visuo-motor pathways in humans revealed by eventrelated fMRI. Experimental Brain Research, 170(4), 472-487. http://doi.org/10.1007/s00221-005-0232-6

Marzi, C. (2010). Asymmetry of interhemispheric communication. Wiley Interdisciplinary Reviews: Cognitive Science, 1(3), 433-438.

Marzi, C. A., Bisiacchi, P., \& Nicoletti, R. (1991). Is interhemispheric transfer of visuomotor information asymmetric? Evidence from a metaanalysis.Neuropsychologia, 29(12), 1163-1177.

Marzi, C. A., Perani, D., Tassinari, G., Colleluori, A., Maravita, A., Miniussi, C., ... Fazio, F. (1999). Pathways of interhemispheric transfer in normals and in a split-brain subject A positron emission tomography study. Experimental Brain Research, 126(4), 451-458.

Mesulam, M.M. (1999). Spatial attention and neglect: parietal, frontal and cingulate contributions to the mental representation and attentional targeting of salient extrapersonal events. Philosophical Transactions of the Royal Society B: Biological Sciences, 354(1387), 1325-1346.

Ocklenburg, S., \& Güntürkün, O. (2012). Hemispheric Asymmetries: The Comparative View. Frontiers in Psychology, 3. http://doi.org/10.3389/fpsyg.2012.00005 
Oleksiak, A., Postma, A., van der Ham, I. J. M., Klink, P. C., \& van Wezel, R. J. A. (2011). A review of lateralization of spatial functioning in nonhuman primates. Brain Research $\quad$ Reviews, 67(1-2), 56-72. http://doi.org/10.1016/j.brainresrev.2010.11.002

Patel, G. H., Yang, D., Jamerson, E. C., Snyder, L. H., Corbetta, M., \& Ferrera, V. P. (2015). Functional evolution of new and expanded attention networks in humans. Proceedings of the National Academy of Sciences, 112(30), 94549459. http://doi.org/10.1073/pnas.1420395112

Pellicano, A., Barna, V., Nicoletti, R., Rubichi, S., \& Marzi, C. A. (2013). Interhemispheric vs. stimulus-response spatial compatibility effects in bimanual reaction times to lateralized visual stimuli. Frontiers in Psychology, 4. http://doi.org/10.3389/fpsyg.2013.00362

Poffenberger, A. T. (1912). Reaction time to retinal stimulation with special reference to the time lost in conduction through nervous centers. Arch Psychol 23:1-73.

Prado, J., Clavagnier, S., Otzenberger, H., Scheiber, C., Kennedy, H., \& Perenin, M. T. (2005). Two cortical systems for reaching in central and peripheral vision. Neuron, 48(5), 849-858.

Premereur, E., Janssen, P., \& Vanduffel, W. (2015). Effector specificity in macaque frontal and parietal cortex. The Journal of Neuroscience, 35(8), 3446-3459.

Raos, V., Umiltá, M. A., Gallese, V., \& Fogassi, L. (2004). Functional properties of grasping-related neurons in the dorsal premotor area F2 of the macaque monkey. Journal of neurophysiology, 92(4), 1990-2002.

Raos, V., Umiltá, M. A., Gallese, V., \& Fogassi, L. (2004). Functional properties of grasping-related neurons in the dorsal premotor area F2 of the macaque monkey. Journal of neurophysiology, 92(4), 1990-2002.

Rilling, J. K., \& Insel, T. R. (1999). Differential expansion of neural projection systems in primate brain evolution. Neuroreport, 10(7), 1453-1459.

Saron, C. D., Foxe, J. J., Schroeder, C. E., \& Vaughan Jr, H. G. (2003). Complexities of interhemispheric communication in sensorimotor tasks revealed by highdensity event-related potential mapping.

Sperry, R. W. (1961). Cerebral organization and behavior. Science,133(3466), 17491757. 
Stamm, J. S., Rosen, S. C., \& Gadotti, A. L. C. I. D. E. S. (1977). Lateralization of functions in the monkey's frontal cortex. Lateralization in the Nervous System. Academic Press, New York, 385-402.

Vallortigara, G. (2006). The evolutionary psychology of left and right: Costs and benefits of lateralization. Dev. Psychobiol. 48, 418-427.

Veale, J. F. (2014). Edinburgh Handedness Inventory - Short Form: A revised version based on confirmatory factor analysis. Laterality: Asymmetries of Body, Brain and Cognition, 19(2), 164-177. http://doi.org/10.1080/1357650X.2013.783045

Wallace, R. J. (1971). SR compatibility and the idea of a response code. Journal of experimental psychology, 88(3), 354.

Weber, B., Treyer, V., Oberholzer, N., Jaermann, T., Boesiger, P., Brugger, P., ... Marzi, C. A. (2005). Attention and interhemispheric transfer: a behavioral and fMRI study. Journal of Cognitive Neuroscience, 17(1), 113-123.

Wey, H.Y., Phillips, K. A., McKay, D. R., Laird, A. R., Kochunov, P., Davis, M. D., ... Fox, P. T. (2014). Multi-region hemispheric specialization differentiates human from nonhuman primate brain function. Brain Structure and Function, 219(6), 2187-2194. http://doi.org/10.1007/s00429-013-0620-9

Zaidel, E., \& Iacoboni, M. (Eds.). (2003). The parallel brain: the cognitive neuroscience of the corpus callosum. Cambridge, Mass: MIT Press. 


\section{General Discussion}

"There are times when those eyes inside your brain stare back at you" Charles Bukowski (1999)

Although Bukowski was right, especially in the context of human deep introspection, the present thesis contributes rather to the idea that our brain "stares back at us" all the time. It does that because our environment is very complex and dynamic and, therefore, it might be of highly adaptive value to read out the reliability of the evidence we base our decisions on, and to use these readouts to learn and plan future behaviors more efficiently.

Considering this reasoning, it is almost intuitive to think that information about being wrong is as important, and not as different, as information about being right. It was, in a way, appealing to me to find out that usually these two "certainty directions" (certainty of being correct and certainty of being incorrect) have been studied separately, because I felt I stumbled upon an interesting problem and my colleagues and I might be the first ones to investigate this in the same experiment. After I started collecting data and performing initial analysis, it became clearer why it is difficult to bring these two metacognitive fields (confidence evaluation and error detection, respectively) together: because it is very not straightforward to find a way to capture the two certainty readout directions, especially without requesting subjects to consciously evaluate their decisions (Boldt and Yeung, 2015) since other cognitive processes might, and probably do, influence certainty-related reports in implicit manner.

The contributions of slope-based measurements to the metacognition field

Although the first chapter is not only a description of the methodological approach I used to capture implicit readouts of certainty of being correct and certainty of being incorrect - since I also propose a framework about how evidence is processed up to the point when these readouts occur -, I am confident (or rather have a high certainty 
of being correct) that the novel slope-based measurements that I created to measure subjects' metacognitive readouts are reliable and highly relevant to psychologists and neuroscientists interested in the cognitive and neural mechanisms of decision-making associated with confidence, changes of mind and error detection, as well as the burgeoning field of reward processing and value-based decision-making.

Moreover, my results provide a new perspective on the formulation and interpretation of certainty scales. Commonly, experimenters did not consider certainty of being incorrect in studies about confidence evaluation, conflating certainty of being incorrect with low certainty of being correct (Fleming and Lau, 2014; Fleming et al., 2012; Heereman et al., 2015; Maniscalco and Lau, 2012; Sandberg et al., 2010). The findings presented here provided extra evidence that it is important to distinguish these two readouts (Fernandez Cruz et al., 2016; Hebart et al., 2014). Furthermore, my approach allows researchers to test the interplay between the two certainty direction readouts by formulating certainty scales using different configurations. For example, right after post-decision wagering (PDW) was published as a method that claims to objectively measures awareness (Persaud et al., 2007), several follow-up papers discussed how different pay-off matrices could modify the interpretation of PDW results, and relate these results to other cognitive processes which do not require awareness, such as loss aversion (Clifford et al., 2008; Schurger and Sher, 2008; Seth et al., 2008). Later, several other research groups concluded that PDW offers the best available metric of confidence due to its elementary neural computations requirements (e.g. Pouget et al., 2016), demonstrating the flexibility of the method. I suggest that this flexibility can now be utilized to test the two certainty directions.

Moreover, in order to isolate PDW trial-specific assessments from assessments which included expected difficulty and psychological biases, I utilized pre-decision wagering trials (PreDW, wagering before the perceptual decision). In the context of our study, PreDW largely constituted a control experiment. Since PDW is a rewardbased decision, it is influenced by the individual's belief that the chosen action maximizes utility (De Martino et al. 2012). As mentioned, variations in the pay-off matrix itself and intersubject variation in reward-related psychological biases, as loss aversion and risk-taking (Byrnes et al., 1999; Fleming and Dolan, 2010), can modify these beliefs. Thus, yet another strength of my method is that PreDW can control for 
the effects of varying subjective probabilities on PDW, serving as an important baseline measurement, without which the interpretations of the main PDW experiment would be ambiguous.

However, the initial data we obtained with preDW is also of interest as a stand-alone study, and warrant further investigations. So far, PreDW has not being a very well explored task, but some variants of pre-decision opt-out task (cf. Hampton, 2001) can be constructed as variants of PreDW. It would be extremely interesting to contrast the PreDW behavior in monkey and human species, and to explore the neural substrate of the PreDW processing. I already had in mind to test if the same areas would encode both pre- and post-decision wagering variables, but due to already very high complexity of the present fMRI task design, this question will have to be addressed in the future studies.

Lastly, since I utilized an intuitive response scale through which subjects reported their certainty readouts in an implicit manner (by trying to maximize their gains and to minimize losses), my method is also relevant for researchers using animal models to investigate metacognitive abilities, as well as to clinical investigators interested in studying metacognitive evaluation in patients with impaired language comprehension. Specifically, it sets stage for the future investigations of metacognitive neural basis that can be conducted using the same techniques across monkeys and humans.

\section{Post-decisional evidence accumulation}

In the chain of decisions that constitute our daily life, our choices can have different outcomes at different timings and with different probabilities. Assessing the likelihood of decisions outcomes is highly valuable in dynamic environments. For that, we need to keep in memory the action we took and reinforce the state-action associations when a reward is given (Doya, 2008). But in the absence of explicit immediate feedback (which is often the case in the real life), adaptive behavior might require the full, or almost full, assessment of the available evidence. In this context, post-decisional evidence is a valuable source of information, and its accumulation has been studied in more detail in recent years (Fleming, 2016; Murphy et al., 2015; van den Berg et al., 2016; Yu et al., 2015). 
In the first chapter, I addressed post-decisional evidence accumulation by comparing subjects' performance during perceptual decisions (d') and during metacognitive decisions (meta-d'). I divided subjects into two groups: the low metacognitive efficiency group (meta-d'<d'), whose performance decreased during PDW (metacognitive judgments) compared to the delayed match-to-sample task (perceptual decision); and the high metacognitive efficiency group (meta-d'>d'), which was formed by subjects who performed better during PDW compared to the perceptual decisions. My results showed that only subjects from the high metacognitive efficiency group were able to read out certainty of being incorrect in order to wager more adaptively. I interpreted these results as a demonstration that meta-d'>d' reflects post-decisional evidence accumulation because - in order to probabilistically detect incorrect decisions - subjects need more information than they had during the decision itself. Otherwise, subjects who detected incorrect choices would not have made an incorrect choice in the first place. Moreover, Yu et al. (2015) showed that post-decisional evidence accumulation is stronger towards the non-selected decision option. In fact, the studies that have been demonstrating the accumulation of information after decisions majorly associate that with error detection or changes of mind (Fleming, 2016; Murphy et al., 2015; van den Berg et al., 2016; Yu et al., 2015).

Nevertheless, there is still some disagreement about the origin and accessibility of the post-decisional evidence used for self-monitoring. Whereas van den Berg et al. (2016) suggested that such input is based on sensory information that was not available during the initial decision due to processing delays, Murphy et al. (2015) proposed that following the decision, top-down signals provide the extra evidence used for post-decisional processes (see also Fleming et al., 2016). Since I used a memorybased perceptual task (delayed match-to-sample task) I am inclined to agree with a third point of view provided by $\mathrm{Yu}$ et al. (2015), which considers that the improvement in certainty readouts accuracy is done, at least partly, by memory-based post-decisional evidence. However, I believe that the mentioned top-down signals are anyway resulting (directly or indirectly) from sensory evidence that was not considered when subjects committed to the reported perceptual decision and, therefore, should also vary as a function of the sensory input. However, this debate will have to be resolved using additional empirical evidence and modeling. 


\section{The neural representations of bi-directional certainty}

After performing the study presented in chapter one, I planned the subsequent fMRI study having in mind a clear and hypothesis-driven scientific question: are both certainty readout directions (certainty of being correct and certainty of being incorrect) encoded together in the brain?

To test that, my colleagues and I created a parametric regressor that was used to identify BOLD signal with bi-directional pattern in the functional activity related to the wagering period of the task. I expected to find, among areas known to encode certainty-related information - such as the anterior prefrontal cortex (aPFC), dorsolateral prefrontal cortex (dlPFC), dorsal anterior cingulate cortex (dACC), intraparietal sulcus (IPS), inferior parietal lobule (IPL), precuneus, posterior cingulate cortex (PCC), ventral striatum and thalamus - at least one area representing the expected bi-directionality. I did not expect to find out that nearly all of them encoded certainty in a bi-directional way.

Since the map with areas encoding bi-directional certainty was very extensive, I performed extra post-hoc tests to guarantee that all the located clusters reliably represented the predicted bi-directional pattern. Of all tested regions-of-interest, as many as 47 clusters survived those rigorous tests.

I interpret these results as a indication that PDW task demands prompted brain-wide encoding of bi-directional certainty information in order to generate the most adaptive behavior (i.e. profit more when correct and avoid large losses when incorrect). Moreover, I suggest that since adaptive wagering reflects the proper assessment of the wager options utility (reflecting certainty only implicitly), the information that led to such flexible behavior should be based on the reliability of the sensory evidence (i.e. certainty) that drives goal-directed behavior. The value-based context of the subjects' behavior is further supported by the activations of reward structures (e.g. basal ganglia) that encoded expected reward linked to perceptual difficulty.

While unidirectional patterns related to the representation of the difficulty levels of the perceptual decisions and to readouts of certainty of being correct were also associated with reward expectation, the bi-directional patterns where too ubiquitous to be generally associated with specific cognitive processes other than certainty readouts. In this context, I return to the action-centered framework that motivated my 
investigations in the first place, as well as to the computational framework that defined the selection of the certainty scale I employed in my experiments.

Therefore, going beyond specific implications for understanding metacognitive function, what does the wide-spread pattern of bi-directional certainty representation mean for a more general view on functional organization of decision-making circuitry? As described in the General Introduction, recent studies demonstrated that instead of being based exclusively on the executive system localized in the frontal lobes, decision-making is also associated with several cortical and subcortical regions related to sensorimotor control, which can be modulated by the perceptual signal strength (Cisek, 2012; Filimon et al., 2013). This "embodied" and action-directed processing, therefore, inherently involves multiple brain areas in relevant information processing. I suggest that this distributed processing will happen especially if the information is not specifically associated with certain cognitive processes, but it is as ubiquitous as certainty readouts. On top of that, I utilized PDW, which includes motivating and relevant behavioral requirements that can be performed using the simple computations that animals employ routinely. Altogether, I suggest that, although it was important to demonstrate that the activity of some "bi-directional brain areas" correlated with subjects' behavior (in particular, IPL and dACC), the ubiquity of bi-directional patterns in the brain is more revealing than these correlations, since it suggests the existence of a global framework that generates adaptive behaviors cohesively. In this global framework, different areas would contribute more closely with specific representations (such areas from the occipital lobule with visual representations, and areas from the motor cortex with motor representations) but working in unison to create a "distributed consensus" encoding of certainty (for review about the distributed consensus see Cisek, 2012).

In this context, comparative studies which investigate the entire animal brain activity in its entirety, as the one presented in the third chapter, seem to be essential for elucidating if such ubiquitous processing is indeed based on simple computations that can be performed by different animal species, as well as how interspecies anatomical and functional differences determine (or limit) each species capacity to behave adaptively.

Finally, it is important to emphasize that while these results demonstrate robust findings across a group of subjects, further analysis should be performed to test task- 
specific connectivity using dynamic causal modeling (DCM) or psychophysiological interaction (PPI) approaches, as well as more novel methods which might be better suited for the event-related data (e.g. BetA Series COrrelation, Göttlich et al., 2015).

\section{Certainty about response requirements}

My initial plan was to investigate introspection and awareness in monkeys and humans, problems with monkey training and delays in the production of MRIcompatible equipment forced me to investigate a simpler, but still very relevant, scientific question: How is interhemispheric processing modified by different levels of certainty of the upcoming response requirements? Thus, instead of investigating the certainty associated with preceding decisions, I addressed the predictability in respect to upcoming visuomotor requirements.

I was able to demonstrate that, in both species, the enhanced uncertainty of the upcoming response increased interhemispheric transfer time, likely as a result of interhemispheric action selection processing and stimulus-response spatial compatibility effects. I also showed, by comparing the brain activity patterns of humans across three tasks with different randomization of response requirements, that such unpredictability did not increase the interhemispheric transfer time per se, but delayed the exchange of relevant information between the hemispheres until the point when a proper response could be implemented without decreasing performance. Lastly, I showed that the time difference between responses which required interhemispheric transfer of information (crossed responses) and responses which could be encoded in one brain hemisphere (uncrossed responses) was longer in humans than in monkeys. There are two possible explanations for this interspecies difference: (1) the interhemispheric communication takes more time in humans because our brain is bigger than the monkey brain, or (2) humans were more successful in delaying crossed responses to guarantee a higher performance. The second interpretation relies on higher performance of humans in the tasks with significant crossed-uncrossed differences (CUD). Another interspecies difference was the presence of negative CUD activations only in monkeys, which might have been related to an imprinted inhibitory mechanism associated with the presentation of cues that instructed monkeys to withhold their manual responses. 
Considering that monkeys were trained for long periods before the actual data collection, I plan to perform an extra experiment in which a low number of overtrained human subjects will perform the exact same task performed by the two monkeys. With this experiment, I aim to elucidate if some differences between humans and monkeys occurred because of intersubject or task variability, or indeed because of behavioral, anatomical or functional organization differences between the two species.

Some problems with the analysis of the data and interpretation of results that I still did not fully overcome are commonly found in the field. Although the first publications about fMRI experiments with awake monkeys date from more than fifteen years ago, only few laboratories perform these experiments as their research focus (Goense et al., 2010), and very few utilize behavioral tasks that require more than either simple fixation or saccades. In fact, only one laboratory so far successfully conducted an analogous reaching (and grasping) study in awake macaques (Nelissen and Vanduffel, 2011). Among the technical problems with monkey fMRI experiments, the main ones are poor signal-to-noise ratio due to the small size of the brain (and thus smaller voxel size) and animal motion.

In my experiments, I employed different techniques to avoid problems resulting from monkey movements. For instance, I checked eye fixation and jaw motions constantly (except for inter-trial intervals) and I required that hands were positioned in a specific resting place during the entire trial. Using advanced event-related approach, I was able to capture expected space and effector tuning-related activations using randomeffects (across sessions) analysis in each monkey individually, and those patterns of activation agreed well between the two monkeys, making it possible to compare these reliable activations to human group data. However, since I was testing speeded reaction time reaches which preceded the trial period in which the relevant BOLD response signal was acquired, the motion caused transient distortions in the magnetic field, which might have contaminated the initial part of the task-related activation. These distortions, while largely negligible for the main effects of space and effector tuning, became more problematic when smaller cognitive effects (such as space $x$ effector interaction, i.e. crossed vs. uncrossed responses) were analyzed. Therefore, I am still exploring the best approaches to optimal statistical modeling and analysis. In particular, better modeling of the confound effects (such as transient distortions) and 
further time-resolved analysis of the BOLD signal timecourses will be necessary to create an objective method to distinguish relevant signal changes from the accompanying noise. Thus, the conclusions about crossed vs. uncrossed neural responses in the two monkeys are currently tentative. Finally, the brain processes related to this experiment might have considerable inter-subject variations, since the analysis of human data also demonstrated high variability across subjects. The next analysis step is to correlate individual behavioral measures with neural activation patterns, across subjects, to distinguish task-specific effects from random noise as the potential sources of this apparent variability.

Nevertheless, whereas I am still improving and expanding my analysis of the monkey fMRI dataset, the knowledge I gained by designing and performing the monkey experiment (and analogous human fMRI experiment) had a positive effect on multiple aspects of the task design and analysis that I employed in the human fMRI study presented in chapter two, since it resulted in reliable and robust results which corroborated my hypothesis. Furthermore, since the visuomotor contingencies employed in the two human studies are largely the same, it would be worthwhile to compare the putative modulation of visual processing and motor preparation signals by the cognitive task demands.

\section{Future directions}

The present thesis described three studies about response selection and certaintyrelated processing, in humans and monkeys. Altogether, it contributes to the notion that our brains, and likely monkey brains, read out certainty continuously, inherently and ubiquitously, and that certainty-related information flexibly modifies the brain processing of the goal-directed behaviors.

Based on that, I believe that it is necessary to perform comparative studies between humans and other animal species (starting from non-human primates, since they are closely related to us) to test how ubiquitous these principles are, and how they contribute to the evolutionary adaptations of the individuals. Therefore, I hope that my findings will motivate further comparative studies on metacognition in our lab and elsewhere, because finding out more about self-monitoring processes seems to be extremely important to understand the brain function as a whole. 


\section{References}

Aboitiz, F., López, J., \& Montiel, J. (2003). Long distance communication in the human brain: timing constraints for inter-hemispheric synchrony and the origin of brain lateralization. Biological Research, 36(1), 89-99.

Alcock, J. (1993). Animal behavior: An evolutionary approach. Sinauer Associates.

Arcaro, M. J., Pinsk, M. A., Li, X., \& Kastner, S. (2011). Visuotopic Organization of Macaque Posterior Parietal Cortex: A Functional Magnetic Resonance Imaging Study. Journal of Neuroscience, 31(6), 2064-2078. http://doi.org/10.1523/JNEUROSCI.3334-10.2011

Boldt, A., \& Yeung, N. (2015). Shared Neural Markers of Decision Confidence and Error Detection. Journal of Neuroscience, 35(8), 3478-3484. http://doi.org/10.1523/JNEUROSCI.0797-14.2015

Bukowski, C. (1999). What Matters Most is How Well You Walk Through the Fire. HarperCollins Publishers Inc. New York.

Byrnes, J. P., Miller, D. C., \& Schafer, W. D. (1999). Gender differences in risk taking: A meta-analysis. Psychological Bulletin, 125(3), 367-383. http://doi.org/10.1037/0033-2909.125.3.367

Charles, L., Van Opstal, F., Marti, S., \& Dehaene, S. (2013). Distinct brain mechanisms for conscious versus subliminal error detection. NeuroImage, 73, 80-94. http://doi.org/10.1016/j.neuroimage.2013.01.054

Cisek, P. (2012). Making decisions through a distributed consensus. Current Opinion in Neurobiology, 22(6), 927-936. http://doi.org/10.1016/j.conb.2012.05.007

Cisek, P., \& Kalaska, J. F. (2010). Neural Mechanisms for Interacting with a World Full of Action Choices. Annual Review of Neuroscience, 33(1), 269-298. http://doi.org/10.1146/annurev.neuro.051508.135409

Clifford, C. W. G., Arabzadeh, E., \& Harris, J. A. (2008). Getting technical about awareness. Trends in Cognitive Sciences, 12(2), 54-58. http://doi.org/10.1016/j.tics.2007.11.009

De Martino, B., Fleming, S. M., Garrett, N., \& Dolan, R. J. (2012). Confidence in value-based choice. Nature Neuroscience, 16(1), 105-110. http://doi.org/10.1038/nn.3279

Fernandez Cruz, A. L., Arango-Muñoz, S., \& Volz, K. G. (2016). Oops, scratch that! Monitoring one's own errors during mental calculation. Cognition, 146, 110 120. http://doi.org/10.1016/j.cognition.2015.09.005 
Filimon, F., Philiastides, M. G., Nelson, J. D., Kloosterman, N. A., \& Heekeren, H. R. (2013). How Embodied Is Perceptual Decision Making? Evidence for Separate Processing of Perceptual and Motor Decisions. Journal of Neuroscience, 33(5), 2121-2136. http://doi.org/10.1523/JNEUROSCI.233412.2013

Fleming, S. M., \& Dolan, R. J. (2010). Effects of loss aversion on post-decision wagering: Implications for measures of awareness. Consciousness and Cognition, 19(1), 352-363. http://doi.org/10.1016/j.concog.2009.11.002

Fleming, S. M., \& Lau, H. C. (2014). How to measure metacognition. Frontiers in Human Neuroscience, 8. http://doi.org/10.3389/fnhum.2014.00443

Fleming, S. M., Huijgen, J., \& Dolan, R. J. (2012). Prefrontal Contributions to Metacognition in Perceptual Decision Making. Journal of Neuroscience, 32(18), 6117-6125. http://doi.org/10.1523/JNEUROSCI.6489-11.2012

Goense, J. B. M., Whittingstall, K., \& Logothetis, N. K. (2010). Functional magnetic resonance imaging of awake behaving macaques. Methods, 50(3), 178-188. http://doi.org/10.1016/j.ymeth.2009.08.003

Göttlich, M., Beyer, F., \& Krämer, U. M. (2015). BASCO: a toolbox for task-related functional connectivity. Frontiers in systems neuroscience, 9.

Hampton, R. R. (2001). Rhesus monkeys know when they remember. Proceedings of the National Academy of Sciences, 98(9), 5359-5362.

Hebart, M. N., Schriever, Y., Donner, T. H., \& Haynes, J.-D. (2016). The Relationship between Perceptual Decision Variables and Confidence in the Human Brain. Cerebral Cortex, 26(1), 118-130. http://doi.org/10.1093/cercor/bhu181

Heereman, J., Walter, H., \& Heekeren, H. R. (2015). A task-independent neural representation of subjective certainty in visual perception. Frontiers in Human Neuroscience, 9. http://doi.org/10.3389/fnhum.2015.00551

Kable, J. W., \& Glimcher, P. W. (2009). The Neurobiology of Decision: Consensus and Controversy. $\quad$ Neuron, 63(6), 733-745. http://doi.org/10.1016/j.neuron.2009.09.003

Kagan, I., Iyer, A., Lindner, A., \& Andersen, R. A. (2010). Space representation for eye movements is more contralateral in monkeys than in humans. Proceedings of the National Academy of Sciences, 107(17), 7933-7938. http://doi.org/10.1073/pnas.1002825107 
Kepecs, A. (2013). The uncertainty of it all. Nature Neuroscience, 16(6), 660-662.

Kepecs, A., \& Mainen, Z. F. (2012). A computational framework for the study of confidence in humans and animals. Philosophical Transactions of the Royal Society B: Biological Sciences, 367(1594), 1322-1337. http://doi.org/10.1098/rstb.2012.0037

Kepecs, A., Uchida, N., Zariwala, H. A., \& Mainen, Z. F. (2008). Neural correlates, computation and behavioural impact of decision confidence. Nature, 455(7210), 227-231. http://doi.org/10.1038/nature07200

Kiani, R., \& Shadlen, M. N. (2009). Representation of Confidence Associated with a Decision by Neurons in the Parietal Cortex. Science, 324(5928), 759-764. http://doi.org/10.1126/science.1169405

Kiani, R., Corthell, L., \& Shadlen, M. N. (2014). Choice Certainty Is Informed by Both Evidence and Decision Time. Neuron, 84(6), 1329-1342. http://doi.org/10.1016/j.neuron.2014.12.015

Klaes, C., Westendorff, S., Chakrabarti, S., \& Gail, A. (2011). Choosing Goals, Not Rules: Deciding among Rule-Based Action Plans. Neuron, 70(3), 536-548. http://doi.org/10.1016/j.neuron.2011.02.053

Knill, D. C., \& Pouget, A. (2004). The Bayesian brain: the role of uncertainty in neural coding and computation. Trends in Neurosciences, 27(12), 712-719. http://doi.org/10.1016/j.tins.2004.10.007

Komura, Y., Nikkuni, A., Hirashima, N., Uetake, T., \& Miyamoto, A. (2013). Responses of pulvinar neurons reflect a subject's confidence in visual categorization. Nature Neuroscience, 16(6), 749-755. http://doi.org/10.1038/nn.3393

Ma, W. J., \& Jazayeri, M. (2014). Neural Coding of Uncertainty and Probability. Annual Review of Neuroscience, 37(1), 205-220. http://doi.org/10.1146/annurev-neuro-071013-014017

Mamassian, P., Landy, M. S., \& Maloney, L. T. (2002). Bayesian modelling of visual perception. Probabilistic Models of the Brain: Perception and Neural Function, 13-36.

Maniscalco, B., \& Lau, H. (2012). A signal detection theoretic approach for estimating metacognitive sensitivity from confidence ratings. Consciousness and Cognition, 21(1), 422-430. http://doi.org/10.1016/j.concog.2011.09.021 
Mantini, D., Gerits, A., Nelissen, K., Durand, J.-B., Joly, O., Simone, L., ... Vanduffel, W. (2011). Default Mode of Brain Function in Monkeys. Journal of Neuroscience, 31(36), 12954-12962. http://doi.org/10.1523/JNEUROSCI.2318-11.2011

Murphy, P. R., Robertson, I. H., Harty, S., \& O’Connell, R. G. (2015). Neural evidence accumulation persists after choice to inform metacognitive judgments. eLife, 4. http://doi.org/10.7554/eLife.11946

Nelissen, K., \& Vanduffel, W. (2011). Grasping-related functional magnetic resonance imaging brain responses in the macaque monkey. The Journal of Neuroscience, 31(22), 8220-8229.

Oleksiak, A., Postma, A., van der Ham, I. J. M., Klink, P. C., \& van Wezel, R. J. A. (2011a). A review of lateralization of spatial functioning in nonhuman primates. Brain Research Reviews, 67(1-2), 56-72. http://doi.org/10.1016/j.brainresrev.2010.11.002

Oleksiak, A., Postma, A., van der Ham, I. J. M., Klink, P. C., \& van Wezel, R. J. A. (2011b). A review of lateralization of spatial functioning in nonhuman primates. Brain Research Reviews, 67(1-2), 56-72. http://doi.org/10.1016/j.brainresrev.2010.11.002

Padoa-Schioppa, C. (2011). Neurobiology of Economic Choice: A Good-Based Model. Annual Review of Neuroscience, 34(1), 333-359. http://doi.org/10.1146/annurev-neuro-061010-113648

Patel, G. H., Yang, D., Jamerson, E. C., Snyder, L. H., Corbetta, M., \& Ferrera, V. P. (2015). Functional evolution of new and expanded attention networks in humans. Proceedings of the National Academy of Sciences, 112(30), 94549459. http://doi.org/10.1073/pnas.1420395112

Peirce, C. S., \& Jastrow, J. (1884). On small differences in sensation.

Persaud, N., McLeod, P., \& Cowey, A. (2007). Post-decision wagering objectively measures awareness. Nature Neuroscience, 10(2), 257-261. http://doi.org/10.1038/nn1840

Poffenberger, A. T. (1912). Reaction time to retinal stimulation with special reference to the time lost in conduction through nervous centers. Arch Psychol 23:173. 
Pouget, A., Drugowitsch, J., \& Kepecs, A. (2016). Confidence and certainty: distinct probabilistic quantities for different goals. Nature Neuroscience, 19(3), 366374. http://doi.org/10.1038/nn.4240

Rabbitt, P. M. (1966). Errors and error correction in choice-response tasks.Journal of experimental psychology, 71(2), 264.

Ratcliff, R., \& McKoon, G. (2008). The diffusion decision model: theory and data for two-choice decision tasks. Neural Computation, 20(4), 873-922.

Resulaj, A., Kiani, R., Wolpert, D. M., \& Shadlen, M. N. (2009). Changes of mind in decision-making. $\quad$ Nature, 261(7261), 266. http://doi.org/10.1038/nature08275

Sandberg, K., Timmermans, B., Overgaard, M., \& Cleeremans, A. (2010). Measuring consciousness: Is one measure better than the other? Consciousness and Cognition, 19(4), 1069-1078. http://doi.org/10.1016/j.concog.2009.12.013

Schurger, A., \& Sher, S. (2008). Awareness, loss aversion, and post-decision wagering. Trends in Cognitive Sciences, 12(6), 209-210. http://doi.org/10.1016/j.tics.2008.02.012

Seth, A. K., Dienes, Z., Cleeremans, A., Overgaard, M., \& Pessoa, L. (2008). Measuring consciousness: relating behavioural and neurophysiological approaches. Trends in Cognitive Sciences, 12(8), 314-321. http://doi.org/10.1016/j.tics.2008.04.008

Shadlen, M. N., Kiani, R., Hanks, T. D., \& Churchland, A. K. (2008). An intentional framework. Better than Conscious, 71-101.

Shallice, T. (1982). Specific impairments of planning. Philosophical Transactions of the Royal Society of London B: Biological Sciences,298(1089), 199-209.

Sherman, P. W. (1988). The levels of analysis. Animal Behaviour, 36(2), 616-619.

Tinbergen, N. (1963). On aims and methods of ethology. Zeitschrift für Tierpsychologie, 20(4), 410-433.

Van den Berg, R., Anandalingam, K., Zylberberg, A., Kiani, R., Shadlen, M. N., \& Wolpert, D. M. (2016). A common mechanism underlies changes of mind about decisions and confidence. Elife, 5, e12192.

Vanduffel, W., Fize, D., Mandeville, J. B., Nelissen, K., Van Hecke, P., Rosen, B. R., ... Orban, G. A. (2001). Visual motion processing investigated using contrast agent-enhanced fMRI in awake behaving monkeys. Neuron, 32(4), 565-577. 
Vanduffel, W., Zhu, Q., \& Orban, G. A. (2014). Monkey Cortex through fMRI Glasses. $\quad$ Neuron, 53(3), 533-550. http://doi.org/10.1016/j.neuron.2014.07.015

Yeung, N., \& Summerfield, C. (2012). Metacognition in human decision-making: confidence and error monitoring. Philosophical Transactions of the Royal Society B: Biological Sciences, 367(1594), 1310-1321. http://doi.org/10.1098/rstb.2011.0416

Yu, S., Pleskac, T. J., \& Zeigenfuse, M. D. (2015). Dynamics of postdecisional processing of confidence. Journal of Experimental Psychology: General, 144(2), 489-510. http://doi.org/10.1037/xge0000062

Zemel, R. S., Dayan, P., \& Pouget, A. (1998). Probabilistic interpretation of population codes. Neural Computation, 10(2), 403-430. 


\section{Curriculum vitae}

\section{Caio Margarido Moreira}

email: caiommoreira@gmail.com

Date of Birth: 08.22.1984

Place of Birth: São Paulo State

Citizenship: Brazilian

\section{EMPLOYMENT HISTORY}

Deutsches Primatenzentrum

Since 2012

Decision and Awareness Group

Laboratory of Cognitive Neuroscience

Teacher of Biology (Elementary School and High School)

Escola Época Positivo

Santa Cruz 544, Centro. Serrana, SP. Brazil

Teacher of Biology (Preparatory Course for University's

admission examination)

Riven Educacional LTDA (Raven Educational)

Florêncio de Abreu 1712, Vila Seixas. Ribeirão Preto, SP. Brazil.

Teacher of Basic Mathematics (Radiology Technician Course) 2006

Instituto Zimmerman de Educação (Zimmerman Institute of

Education)

Avenida do Café 956, Vila Tibério. Ribeirão Preto, SP. Brazil.

\section{EDUCATIONAL HISTORY}

Graduation in Behavior and Cognition

$\mathrm{PhD}$ Behavior and Cognition Program

University of Goettingen

Specialization in Public Understanding of Science and Health: $\quad 2009$ - 2010

Neurosciences

Faculty of Medical Sciences

University of Campinas, Campinas, SP. Brazil.

Graduate in Experimental Psychology (Master's Degree)

Department of Experimental Psychology, Institute of Psychology

University of São Paulo, São Paulo, SP. Brazil.

Bachelor in Biological Sciences

$2003-2007$

Faculty of Philosophy, Sciences and Letters of Ribeirão Preto

University of São Paulo, São Paulo, SP. Brazil. 


\section{GRANTS}

Scientific Initiation Fellowship by CNPQ (National Counsel of $2004-2006$ Technological and Scientific Development/Brazil) - \$4000

I investigated the neurobiology of fear and anxiety in the Laboratory of Neuropsychopharmacology, Department of Psychology (University of São Paulo). This experience included the study of animal behavior utilizing animal models for rats and gave me familiarity to work with specialized devices used to perform histological analyses and neurochemical analyses (e.g. High-Performance Liquid Chromatography).

Master's Fellowship by FAPESP (The State of São Paulo Research Foundation) - \$12000

I studied the physiology of stress and reproduction in the Laboratory of Cognitive Ethology, Institute of Psychology (University of São Paulo). I learned to study the behavior of wild animals (monkeys) and how to work with devices and assays (Enzyme Imunoassay) utilized to analyze hormone metabolites.

PhD Fellowship by SwB (Science without Borders) CNPq Since 2012

I'm studying decision-making associated with certainty-related processes in humans and non-human primates using fMRI. These studies are being performed at the Decision and Awareness Group of the German Primate Center.

\section{PUBLICATIONS}

Moreira, C. M., Rollwage, M., Kaduk, K., Wilke, M., Kagan, I. Certainty bidirectionality and the efficient use of metacognition. Submitted to Cognition

Moreira, C. M., dos Santos, L. P., de Souza, M. B. C., Izar, P. Variation of 2016 glucocorticoid metabolite levels is associated with survival demands in immature and reproductive demands in adult wild black capuchins (Sapajus nigritus). Accepted in the International Journal of Psychological Research

Carvalho, M.C., Moreira, C.M., Zanoveli, J.M., Brandão, M.L. Central, but 2010 not basolateral, amygdala involvement in the anxiolytic-like effects of midazolam in rats in the elevated plus maze. Journal of Psychopharmacology (Oxford), v. 26, p. 543-554, 2012.

Moreira, C. M: Não fui eu, foi meu cérebro! Viver Mente \& Cérebro. v. 211. p. 30-35. ("It wasn't me, it was my brain!". A science outreach paper about decision-making published in a magazine of the Scientific American group).

Moreira, C. M., Masson, S., Carvalho, M.C., Brandão, M.L. Exploratory behaviour of rats in the elevated plus-maze is differentially sensitive to inactivation of the basolateral and central amygdaloid nuclei. Brain Research Bulletin, v. 71, p. 466-474. 


\section{SELECTED COURSES}

Winter School in "The neuroscience of decision making" of the 2015 Federation of European Neuroscience Society.

Obergurgl, Austria.

\section{SELECTED CONFERENCES (POSTERS)}

Post-decision wagering reveals both perceptual confidence and error detection awareness.

Moreira, C. M., Rollwage, M., Kaduk, K., Wilke, M., Kagan, I.

In: The 19th Annual Meeting of the ASSC, 2015, Paris.

Interhemispheric brain processing during action selection

Moreira, C. M., Wilke, M. Kagan, I.

In: OHBM's 2015 Annual Meeting, 2015, Honolulu.

Interhemispheric brain processing during action selection in human and 2015 non-human primates.

Moreira, C. M. \& Kagan, I.

In: Primate Neurobiology Meeting, 2015, Göttingen.

Science Circle: A TV-inspired talk show for science awareness starring teenagers, artists and scientists

Bueno, L. S.; JR, Ruggiero, R. N.; Ávila, M. A. V.; Daharem, G. C.;

Guizzo, R..; Lopes-Aguiar C., Moreira, C. M.; Souza-Oliveira, G.; Souza-

Pinto, L. F. S.; Vita, J. P.; Garcia-Cairasco, N.

In: $47^{\text {th }}$ Neuroscience Annual Meeting, San Diego - EUA

Seasonal hormonal changes in wild black-capped capuchin (Cebus

2009 nigritus)

Moreira, C. M.; dos Santos, L. P. C.; Souza, M. B. C.; Izar, P.

In: Animal Behavior Society Annual Meeting, Pirinópolis - Brazil

Resting less to explore more! An ontogenetic study of wild black-capped 2009 capuchins (Cebus nigritus) behavioral ecology

dos Santos, L. P. C.; Moreira, C. M.; Izar, P.

In: 14th Biennial Meeting of International Society for Comparative

Psychology, Buenos Aires - Argentina

The central and basolateral amygdala nuclei have distinct roles in the regulation of exploratory behavior of rats in the elevated plus-maze Moreira, C. M.; Masson, S.; Carvalho, M.C.; Brandão, M.L.

In: XXI Annual Meeting of the Federation of Societies of Experimental Biology, Águas de Lindóia - Brazil. 
AWARDS

Honorable mention at $13^{\text {th }}$ International Symposium of Scientific

Initiation of University of São Paulo.

São Paulo, SP. Brazil.

\section{TEACHING EXPERIENCE}

Besides the formal classes I have been volunteering, since 2002, in community preparatory courses to help students who had completed secondary education in public schools to be accepted in the highest quality Brazilian Universities. During my undergraduate course, two friends and I founded a similar preparatory course inside the University of São Paulo called PEIC (Community Interdisciplinary Educational Project). At PEIC, I was Director and Academic Advisor besides teaching. PEIC still exists nowadays, and is established as a successful educational project that has been helping to get students admitted in Brazilian Universities every year.

During my Masters, I was Teaching Assistant in Ethology (2008-2009) and Motivation and Emotion (2009) undergraduate courses.

\section{PUBLIC UNDERSTANDING OF SCIENCE}

Since 2008, I have been participating of a group that intends to help laypeople to understand science (Green Group). This group is formed by neuroscience graduate students, journalists, advertisers, pedagogues and artists, and together we coordinate a number of projects, such as an experimental TV-inspired talk show released through the Internet (YouTube - http://www.youtube.com/grupoverderp) and through DVDs, that is distributed free in public schools of Ribeirão Preto city, São Paulo State Brazil. The Science Circle is centered on a playful interview with a guest researcher who answers to questions asked by local teenager students in a simplified way. In order to motivate students to ask questions, preparatory classes are taught by the graduate students about general topics of the research led by the $\mathrm{PhD}$ to be interviewed.

During my Masters, I took a Specialization for Public Understanding of Science and Health, where I learned journalistic methods to divulge science.

\section{EVENTS ORGANIZATION}

First European Student Conference on Behavior and Cognition

Goettingen, Germany

First Neuroscience and Education Forum 
CULTURAL ACTIVITIES

Instrumental Music Band: Eletroeco - Instrumental band that creates music mixing Brazilian rhythms and rock n' roll (http://www.myspace.com/eletroeco). 